
and nutrient balances in aquaponics 


\section{Propositions}

1. Real-time measurements of soluble phosphorus, calcium and sodium from fish excretions can significantly improve nutrient efficiency in aquaponics.

(this thesis)

2. In aquaponics, calcium, magnesium, and sodium concentrations from fish water limit nutrient reuse by plants.

(this thesis)

3. The uncertainty principle implies that it is impossible to simultaneously improve the expression of a desired genetic trait, and the continuity of a species.

4. The 24 Chinese solar terms provide opportunities for more detailed insights in model-based studies on agriculture, compared to the standard four seasons.

5. Open-source code helps the transition from intermediary-based review of stagnant documents, towards community-based evolving science.

6. The main advantage of urban agriculture over industrial food production is local community involvement.

7. There is a strong need for incentives to stimulate communication with the general public in the personal development of scientists.

Propositions belonging to the thesis entitled:

'Modelling and identification of water and nutrient balances in aquaponics'

Daniel Reyes Lastiri

Wageningen, 07 September 2021 


\section{Modelling and identification of water and nutrient balances in aquaponics}

Daniel Reyes Lastiri 


\section{Thesis committee}

\section{Promotor}

Prof. Dr Karel J. Keesman

Personal chair at Applied Mathematics

Wageningen University \& Research

\section{Co-promotors}

Prof. Dr Huub H.H.M. Rijnaarts

Professor of Environmental Technology

Wageningen University \& Research

Dr. Hans J. Cappon

Research Scientist, Environmental Technology

Wageningen University \& Research

Professor of Water Technology

HZ University of Applied Sciences

\section{Other members}

Prof. Dr GBM Heuvelink, Wageningen University \& Research

Prof. Dr. Habil. T Rauschenbach, Fraunhofer Institute of Optronics, Germany

Prof. Dr AJ Murk, Marine Animal Ecology, Wageningen University \& Research

Dr H Miedema, Wetsus, The Netherlands

This research was conducted under the auspices of the Graduate School Wageningen Institute for Environment and Climate Research (WIMEK), and the Research School for Socio-Economic and Natural Sciences of the Environment (SENSE). 


\title{
Modelling and identification of water and nutrient balances in aquaponics
}

\author{
Daniel Reyes Lastiri
}

\section{Thesis}

submitted in fulfilment of the requirements for the degree of doctor at Wageningen University

by the authority of the Rector Magnificus,

Prof. Dr A.P.J. Mol, in the presence of the

Thesis Committee appointed by the Academic Board to be defended in public on Tuesday 7 September 2021 at 11 a.m. in the Aula. 
Daniel Reyes Lastiri

Modelling and identification of water and nutrient balances in aquaponics, 154 pages

PhD thesis, Wageningen University, Wageningen, the Netherlands (2021) With references, with summary in English

ISBN: 978-94-6395-851-6

DOI: https://doi.org/10.18174/548235 
"We will someday be the ancients, and we can choose what that will mean."

Michael Stevens

"If you want common knowledge to be true, you have to let true knowledge be common."

Joe Hanson 



\section{Contents}

1 General introduction $\quad 1$

1.1 Background. Global context of food production . . . . . . . . . 2

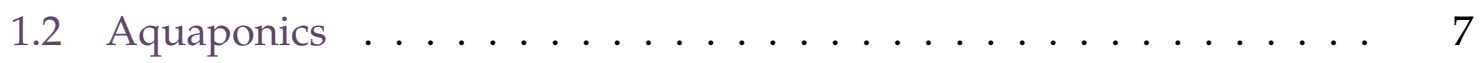

1.3 Mathematical models in aquaponics . . . . . . . . . . . . . . 12

1.4 Research objective . . . . . . . . . . . . . . . . . . . . . 14

1.5 Contents of this thesis . . . . . . . . . . . . 15

2 Model of an aquaponic system for minimised water, energy and nitrogen re$\begin{array}{ll}\text { quirements } & \mathbf{1 7}\end{array}$

2.1 Introduction . . . . . . . . . . . . . . . 18

2.2 Methods . . . . . . . . . . . . . . . . . . . 18

2.3 Results and discussion . . . . . . . . . . . . . . . 24

2.4 Conclusions . . . . . . . . . . . . . . . . . 28

3 Model-based management strategy for resource efficient design and opera$\begin{array}{ll}\text { tion of an aquaponic system } & 29\end{array}$

3.1 Introduction . . . . . . . . . . . . . . . . . 30

3.2 Methods ............................ 31

3.3 Results and discussion . . . . . . . . . . . . . . . . 39

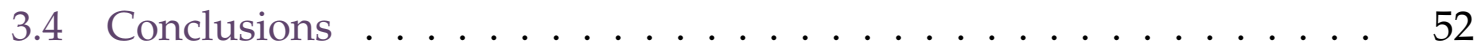

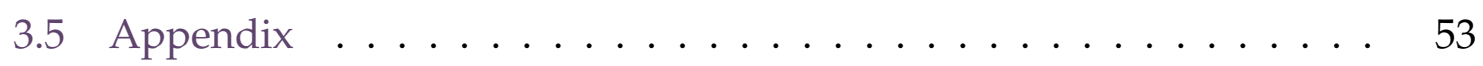

4 Set-membership parameter estimation based on Voronoi vertices 59

4.1 Introduction . . . . . . . . . . . . . . . 60

4.2 Methods .......................... 63

4.3 Results and discussion . . . . . . . . . . . . 71

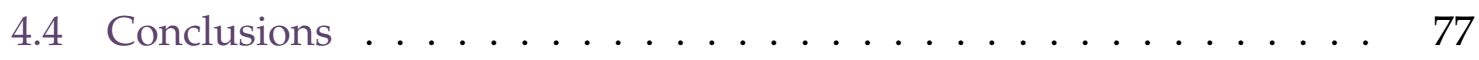

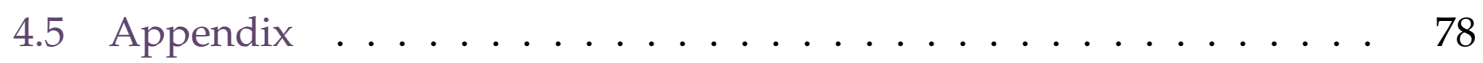

5 Set-membership uncertainty assessment of a mathematical model for nutrient balances in fish growth and excretions. 83

5.1 Introduction . . . . . . . . . . . . . . . . . . 84

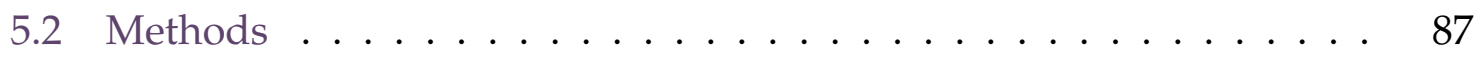

5.3 Results and discussion . . . . . . . . . . . . . . . 96 
viii

5.4 Conclusions . . . . . . . . . . . . . . . . . . . . 104

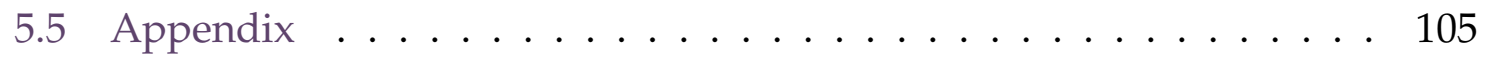

6 General discussion $\quad 109$

6.1 Research questions . . . . . . . . . . . . . . . . . 109

6.2 A framework for modelling growth and nutrient balances in biological production systems . . . . . . . . . . . . . . . . . 116

6.3 Socio-economic challenges for aquaponics and local food production 119

$\begin{array}{ll}\text { Bibliography } & 123\end{array}$

$\begin{array}{ll}\text { Summary } & 137\end{array}$

$\begin{array}{ll}\text { Acknowledgements } & 141\end{array}$ 


\section{Chapter 1}

\section{General introduction}

Aquaponics combines production of aquatic species (aquaculture) with soilless plant production (hydroponics) (Fig. 1.1).

This thesis investigates the dynamics of water and nutrient balances in aquaponics, through the development of mathematical models supported by demonstration aquaponic systems. The studies presented in this thesis aim at improving our understanding of aquaponics, towards an efficient use of resources. A knowledge-based approach for the implementation of aquaponics can contribute to the transition from centralized, market-oriented global food trade structures, towards local, resourceefficient and sustainable food production systems.

The present chapter is a general introduction to the thesis, which provides:

- A brief summary of the current context of food production systems, their limitations and negative impacts.

- An explanation of aquaponics as a sustainable alternative towards local food production.

- The role of mathematical models in the development of aquaponics.

- The objective and research questions of this thesis .

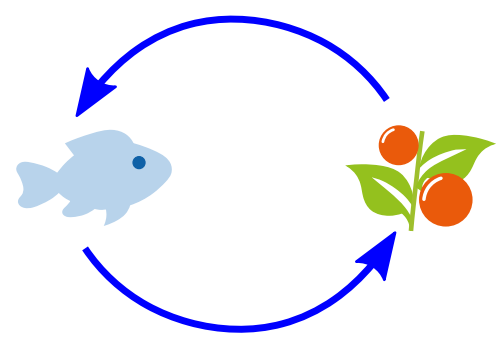

FIGURE 1.1: In aquaponics, water rich in nutrients from aquatic species is reused to fertilise plants, which in turn help cleaning the water for the aquatic species. 


\subsection{Background. Global context of food production}

The rise of industrial agriculture provided the means for an increase in the share of global urban population, from less than $10 \%$ in 1800 , to $13 \%$ in 1900 , to $50 \%$ in 1950 , exceeding the halfway mark for the first time in 2008, and with a predicted $60 \%$ by 2030 (Sachs, 2008).

However, migration from rural to urban areas has not only been motivated by rising farm productivity or better quality of life in cities, but also by poverty and hunger in the countryside (Sachs, 2008). As a result, the global context of food production involves large inequalities and external impacts which remain unsolved.

This section elaborates on the aforementioned impacts, based on food production and trade, water use, and fish production.

\subsubsection{Food production and trade}

Since the 1940s, industrial agriculture was seen as a technology that would allow to feed a rapidly growing population around the world. Therefore, agricultural development policies have focused on monoculture, investing in high potential areas, mechanization, irrigation and crop specialization, with a special interest on marketable and export crops, all part of the so-called green revolution. While these technologies have lifted a large part of the world population from hunger and starvation, they have has also led to the rise of food dependence (Brown, 2001) (see Table 1.1).

Furthermore, recent studies show that previous projections for food security based on industrial agriculture were often overly optimistic (Grassini et al., 2013). Common projections of exponential growth in food production are not feasible because farm yields eventually reach biophysical limits. Instead, historical linear growth trends have been observed. Past increases in production were driven by the adoption of green revolution technologies, such as high yield crops, pesticides, and fertilizers; all one-time innovations, which means that the production increase in a specific land cannot be repeated. Evidence also indicates that production growth has reached a plateau for rice, wheat and maize in areas that account for $31 \%$ of the global production.

Additionally, wide-spread implementation of industrial agriculture has resulted in several environmental (Table 1.2) and social (Table 1.3) impacts. 
TABLE 1.1: Grain production and export [Mt].

The largest exporters (world grain suppliers) are also some of the largest producers, reflecting the global food dependence on a few regions. Data from USDA (2018).

\begin{tabular}{|c|c|c|c|c|c|c|c|c|}
\hline & \multicolumn{2}{|l|}{ Corn } & \multicolumn{3}{|c|}{ Milled rice } & \multicolumn{3}{|c|}{ Wheat } \\
\hline & Prod. & Export & & Prod. & Export & & Prod. & Export \\
\hline World & 1075.2 & 141.7 & World & 486.2 & 47.7 & World & 750.5 & 182.2 \\
\hline US & 384.8 & 55.5 & China & 145.0 & 1.2 & EU & 145.2 & 27.3 \\
\hline China & 219.6 & n.a. & India & 109.7 & 12.3 & China & 128.8 & n.a. \\
\hline Brazil & 98.5 & 19.8 & Indonesia & 36.9 & n.a. & India & 87.0 & n.a. \\
\hline EU & 61.5 & 2.2 & Bangladesh & 34.6 & n.a. & Russia & 72.5 & 27.8 \\
\hline Argentina & 41.0 & 23.0 & Vietnam & 27.4 & 6.5 & US & 62.8 & 29.5 \\
\hline & & & Thailand & 19.2 & 11.6 & Canada & 31.7 & 20.2 \\
\hline & & & Myanmar & 12.7 & 3.4 & Australia & 30.4 & 22.1 \\
\hline & & & US & 7.1 & 3.4 & & & \\
\hline & & & Pakistan & 6.9 & 3.6 & & & \\
\hline
\end{tabular}

TABLE 1.2: Environmental impacts from industrial agriculture.

Information from Langer (1996), FAO (2011), Clay (2013), Penagos et al. (2003),

Smith et al. (2014), Khoury et al. (2014), and Breitburg et al. (2018).

\begin{tabular}{|c|c|}
\hline Impact & Details \\
\hline Greenhouse gases (GHG) & $\begin{array}{l}\text { - Largest emitter of GHG } \\
\left.\text { (5.0 - 5.8 Gt } \mathrm{CO}_{2}-e q \mathrm{yr}^{-1} \text { in } 2000-2010\right) \text {. } \\
-56 \% \text { of global anthropogenic non- } \mathrm{CO}_{2} \text { greenhouse gases, } \\
35 \% \text { of which are from animal enteric fermentation. }\end{array}$ \\
\hline Land use and soil & $\begin{array}{l}\text { - Accounts for } 11 \% \text { of the land, displacing natural habitats. } \\
\text { (60\% of agricultural land used for fodders and pastures) } \\
\text { - Decreased nutrient availability in soil. } \\
\text { - Erosion and compaction. }\end{array}$ \\
\hline Fresh water & $\begin{array}{l}\text { - Agricultural runoff pollutes freshwater sources and coasts: } \\
\text { - Estrogen in water mainly originates from livestock waste. } \\
\text { - } \mathrm{O}_{2} \text { levels in coastal waters decrease. }\end{array}$ \\
\hline Biodiversity & $\begin{array}{l}\text { - Monoculture practices alter insect population: } \\
\text { - Increased densities of herbivores } \\
\text { - Decreased densities of predators and parasitoids } \\
\text { - Reduced genetic diversity of crops, } \\
\text { with less ability to defend from pests } \\
\text { - Marine and freshwater species damaged by } \\
\text { runoff and its consequences. }\end{array}$ \\
\hline
\end{tabular}


TABLE 1.3: Social impacts from industrial agriculture. Information from Kearney (2010), Khoury et al. (2014), and FAO (2011).

\begin{tabular}{ll}
\hline Impact & Details \\
\hline Food security & - Increased food dependence, a potential threat to food security \\
Dietary diversity & - Globally homogeneous food supply. \\
& - National supply richer in calories, but with poorer local diversity. \\
& - Agricultural runoff pollutes freshwater sources and coasts: \\
Health & - Increased proportions of energy-dense, but nutrient-poor foods \\
& - Rising rates of obesity and cardiovascular disease \\
Inequality & - Policies benefited farmers with land and water. \\
& - Small producers locked in poverty trap.
\end{tabular}

\subsubsection{Water use}

Agriculture accounts for $70 \%$ of all water withdrawn from aquifers, streams and lakes (FAO, 2011). This water is often contaminated by plant protection products and nutrient run-off from fertilizer, which have large negative impacts on fresh and marine water biodiversity (Clay, 2013). These impacts are exacerbated by the low investment in vulnerable regions, where poverty and food insecurity are prevalent, and the risk of water and land degradation is high (FAO, 2011).

Hoekstra and Mekonnen (2012) estimated the ground and surface water footprint of national consumption per capita. They found that many industrialized countries, particularly in Northern Europe, have a large share of external footprint, i.e. they mainly consume products that require water in their production process elsewhere (Fig. 1.2). From the global water footprint, $24 \%$ was estimated to be meant for export. From those virtual water exports, 91\% was related to trade in agricultural products.

The water footprint between Mexico and US illustrates the complexity in the distribution of agricultural impacts in the context of global trade. Mexico stands out as the largest virtual water importer in the world, which can be explained by the trade of manufactured goods. The water footprint was estimated for the period 1996-2005. In 1994, the North America Free Trade Agreement (NAFTA) was implemented. From 1994 to 2007, the US trade balance with Mexico decreased from 1.3 (net exporter) to -74.6 billion USD (net importer) (Palley, 2009). Maquila industries in Mexico grew largely in the years soon after the implementation of NAFTA, frequently importing raw materials from US, exporting the manufactured products to 
the US, and even importing back brand products. (Buitelaar and Pérez, 2000). ${ }^{1}$

Though extensive, the water footprint estimation by Hoekstra and Mekonnen (2012) was limited to tracing the origin of products by one step. However, the complexity of global agricultural trade makes it very difficult to locate and identify the origin and end result of impacts on water and the associated nutrient flows from production and discharge of fertilizer.

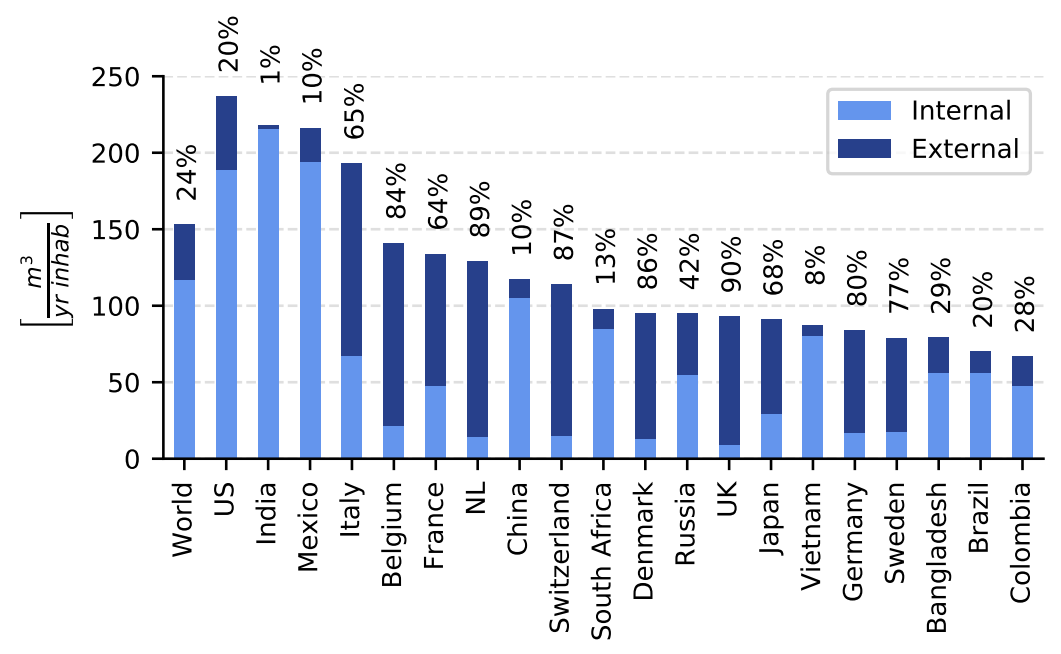

FIGURE 1.2: Water footprint of selected countries, indicating their external share. Elaborated with data from Hoekstra and Mekonnen (2012).

\subsubsection{Fish production}

Even small quantities of fish have a positive nutritional impact on plant-based diets. It provides benefits against cardiovascular disease and can correct unbalanced diets, countering obesity. Furthermore, fish and crustaceans are the most efficient protein producing animals for human consumption (Table 1.4) (Brown, 2001). In 2013 , fish consumption constituted $17 \%$ of animal protein intake and $6.7 \%$ of total protein intake. Annual per capita consumption of fish has grown steadily in developing and low income countries, lowering the gap with industrialized countries (FAO, 2016). Therefore, fish constitutes an option for the shift in sources of protein to more sustainable options (known as the protein transition).

However, by $2013,31 \%$ of the global fish stocks were overfished, and only $10 \%$ of the underfished stocks are able to support increased harvesting (FAO, 2016). The ten most productive species account for $27 \%$ of fisheries production, but most of their stocks are fully fished, with no possibility for reproduction. Regions like the

\footnotetext{
${ }^{1}$ Maquilar is a verb in Mexican Spanish that means 'the action of importing raw materials to process and export them'.
} 
Mediterranean and the Black Sea experienced a production drop by one third since 2007.

As an alternative, aquaculture has increased since the 1980s (FAO, 2016). In 2014, the contribution of aquaculture for human consumption surpassed that of wild-caught fish. World aquaculture production of fish accounted for $44 \%$ of total production (including non-food uses). However, aquaculture production is already experiencing centralization, with China, Indonesia and India accounting for $75 \%$ of global production (Table 1.5). China alone accounts for $61 \%$ of global aquaculture.

TABLE 1.4: Protein efficiency (feed conversion ratio) in animal production.

Data from Brown (2001).

\begin{tabular}{lc}
\hline & $\begin{array}{c}\text { Feed conversion ratio (FCR) } \\
{[k g \text {-feed per } k g \text {-weight gain }]}\end{array}$ \\
\hline Cattle & 7 \\
Pig & 4 \\
Chicken & 2 \\
Fish & $<2$ \\
\hline
\end{tabular}

TABLE 1.5: Aquatic animal production from capture and aquaculture [Mt] (including non-food production). Data from (FAO, 2016)

\begin{tabular}{lrrrrr}
\hline & $\begin{array}{l}\text { Marine } \\
\text { capture }\end{array}$ & $\begin{array}{c}\text { Inland } \\
\text { capture }\end{array}$ & Aquaculture & Total & $\begin{array}{c}\text { Aquaculture } \\
\text { contribution } \\
(\%)\end{array}$ \\
\hline World total & 81.545 & 11.896 & 73.784 & 167.225 & 44.1 \\
China & 14.811 & 2.295 & 45.469 & 62.576 & 72.7 \\
Indonesia & 6.017 & 0.420 & 4.254 & 10.691 & 39.8 \\
US & 4.954 & 0.000 & 0.426 & 5.380 & 7.9 \\
Russia & 4.001 & 0.225 & & & \\
Japan & 3.630 & & 0.657 & 4.287 & 15.3 \\
Peru & 3.549 & & & & \\
India & 3.419 & 1.300 & 4.881 & 9.600 & 50.8 \\
Vietnam & 2.711 & 0.208 & 3.397 & 6.316 & 53.8 \\
Myanmar & 2.702 & 1.381 & 0.962 & 5.045 & 19.1 \\
Norway & 2.301 & & 1.333 & & \\
Chile & 2.175 & & 1.215 & & \\
Philippines & 2.137 & 0.214 & 0.788 & 3.139 & 25.1 \\
R. of Korea & 1.719 & 0.000 & 0.480 & 2.199 & 21.8 \\
Thailand & 1.560 & 0.210 & 0.935 & 2.704 & 34.6 \\
Malaysia & 1.458 & & 0.276 & & \\
Mexico & 1.396 & & & & \\
Morocco & 1.350 & & & & \\
Spain & 1.104 & & 0.282 & & \\
\hline
\end{tabular}


In 2014 and 2015, the EU was the largest single market for fish imports, but aquaculture accounted for less than $1 \%$ of its fish production (except Norway).

The rise of industrial-scale aquacultural production has raised awareness on its environmental and social impacts. Marine and coastal aquaculture (extensive, open systems) have effects on water quality and increased antibiotic resistance in their immediate environment (Yeats et al., 2005; Armstrong et al., 2005). In particular, farmed salmon has been reported to contain environmental and antibiotic contaminants in concentrations higher than wild salmon and other farmed species like tilapia and shrimp (Hites et al., 2004; Hamilton et al., 2005; Van Leeuwen et al., 2009).

Inland aquaculture, particularly in recirculating systems (intensive, closed systems), provides ways to control water quality, decrease exposure to environmental contaminants, and therefore decrease the use of antibiotics. Recirculating water leads to accumulation of waste. Therefore, technologies for management and reuse of waste from aquaculture are implemented and in continued development (Timmons and Ebeling, 2010).

In summary, industrial agriculture based on global trade is not sustainable. Current production methods are reaching biophysical limits and they result in large water use, nutrient waste, environmental impacts and social costs that are often externalized to vulnerable regions. Therefore, sustainable alternatives for local food production are necessary. These alternatives should aim at lower water and nutrient use, with decreased environmental impacts, while maintaining and improving present nutritional and social opportunities for the communities involved. One such alternative is aquaponics.

\subsection{Aquaponics}

Aquaponics is a technology that combines production of aquatic species (aquaculture) with production of plants without soil (hydroponics). In an aquaponic system, nutrients in the excretions of aquatic species help fertilizing plants, and the nutrient uptake by plants helps improving water quality for the aquatic species.

Scientific research on aquaponics was introduced in the 1970s. Wastewater treatment technologies were tested at inland fish farms to improve water quality in the fish tanks. In the same decade, plant production using discharge from municipal and industrial waste-water was being tested, aiming for improved nutrient recovery (Naegel, 1977). It was then proposed to use hydroponics as a means to recover nutrients from discharged recirculating water in fish farms (Naegel, 1977; Lewis et al., 1978). 
The principle of aquaponics, however, is not new. Combining aquatic species and horticulture to benefit from the nutrient cycles in nature has been long known, for example, as rice fields with fish culture in South-East Asia (Coche, 1967), and chinampas by the Aztecs (Turcios and Papenbrock, 2014).

Home-scale aquaponics started growing during the 1990s, with a focus on selfconsumption of fish and plants. These home-scale systems have a low-cost approach and benefit from recycled components, requiring little monitoring and maintenance. Early commercial enterprises were based on selling aquaponic kits (Bernstein, 2011).

To this day, aquaponics remains largely a home-scale technology, with $70 \%$ of the commercial systems made by self-construction or pre-manufactures kits. Profitability in aquaponics largely depends on diversifying revenue by providing services and training. Plants are the main source of revenue, probably due to the higher biomass conversion ratio and growth rates, allowing for multiple plantings in the same year. In the US, plants commonly grown include basil and other herbs, salad greens, lettuce, kale and tomato, with median production ranging from 45 to 226 $k g \mathrm{yr}^{-1}$. Fish commonly grown include tilapia, ornamental species and catfish, with median production ranging from 23 to $45 \mathrm{~kg} \mathrm{yr}^{-1}$. The average of aquaponic system size is 0.01 ha with 10,300 $\mathrm{L}$ in water volume (Love et al., 2015).

Scaling up aquaponics for commercialization presents several challenges for design, monitoring and maintenance. The main challenge consists in balancing optimal conditions required by multiple species: aquatic organisms, plants and beneficial micro-organisms (Suhl et al., 2016). To overcome this challenge, decoupled aquaponic systems have been proposed, consisting of two separate recirculating water loops (Fig. 1.3) (Kloas et al., 2015). The production loop for fish operates independently from the production loop for plants. In this way, each loop can operate with its optimal conditions, implementing technologies that have proven commercial success. Nutrient-rich water from fish can be supplied to the plants continuously or at selected times, reducing fertilizer requirements. Similarly, water from the plants can be recovered and supplied to the fish, reducing water demand.

\subsubsection{Recirculating aquaculture systems (RAS)}

RAS produce aquatic species by (partially) reusing water after treatment, allowing for a controlled environment with predictable growth rates and harvesting schedules (Timmons and Ebeling, 2010).

Current RAS operate with $0.1-1.0 \mathrm{~m}^{3}$ water per $\mathrm{kg}$ feed. New technologies used in 'next generation' RAS could decrease water use even further (Verdegem et al., 2006). Generally, an inland RAS consists of units for: 1) culture, 2) solids removal and 3) biological filtration (Timmons and Ebeling, 2010). 


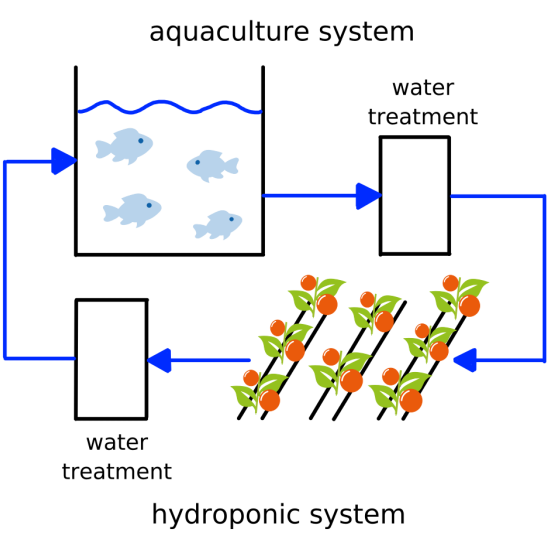

A) coupled aquaponic system

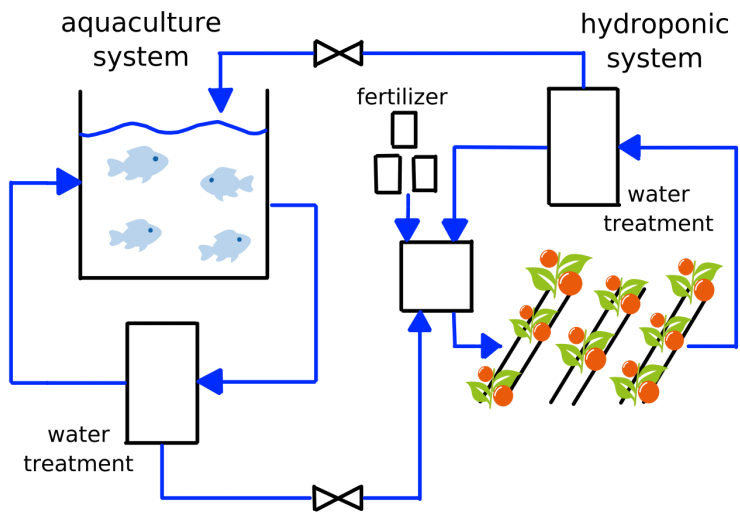

B) decoupled aquaponic system

FIGURE 1.3: Comparison of coupled and decoupled aquaponic system configurations. The coupled configuration (A) has a single water circulation loop for fish and plants. The decoupled configuration (B) allows for operating the fish and plants loops independently, with their own optimal conditions, and water is exchanged between loops in a controlled way.

Culture units. These consist of fish tanks or raceways. Different geometries are available, but circular tanks are preferred because they improve water mixing and solids removal when compared to plug flow tanks (Summerfelt et al., 2004). Using dual drain tanks can further increase solids removal, with one bottom centre drain for solids and one main elevated drain (Summerfelt et al., 2000). Continuous production strategies are preferred over batch production, because they keep the RAS just below its maximum carrying capacity (Timmons and Ebeling, 2010).

Solids removal units. Solid waste consists mainly of faeces and uneaten feed. It can be removed by gravity sedimentation or by filtration. Common sedimentation units are settling tanks, which are inexpensive but require high residence times (low flow rates or high volume), and have poor removal efficiency of small solids. Common filtration units are microscreen filters, such as drum filters, which require minimal labour and small volume, and can operate at high flow rates. Filtration followed by settling has been reported to increase removal efficiency (Cripps and Bergheim, 2000).

Biological filtration units. Fish excrete nitrogenous waste products through gills, urine and faeces. Ammonia is particularly toxic for fish, and therefore must be removed from the recirculating water. This removal is accomplished in a biological filter, where aerobic autotrophic bacteria oxidise ammonia to nitrite and then to nitrate (nitrification) (Timmons and Ebeling, 2010). Biofilters operate by maximizing the surface area that facilitates nitrification. Common types of biofilters are trickling towers and moving beds. 
Other processes in RAS include aeration, using equipment like U-tubes and aeration cones; and disinfection, using UV or ozone (Timmons and Ebeling, 2010).

Research on 'next generation' RAS studies additional processes for waste management, before discharging water to the environment. Examples include denitrification in anaerobic reactors, to convert nitrate into gaseous nitrogen; sludge thickening technologies like geotextile bags (Martins et al., 2010); remineralization to recover insoluble organic nutrients using an upflow anaerobic sludge bed (UASB) reactor (Goddek et al., 2016); and, last but not least, reusing nutrients recovered as fertilizer in aquaponics, the subject of this thesis.

\subsubsection{Greenhouse hydroponic horticulture}

Hydroponics is a technique for plant production without soil. ${ }^{1}$ Experiments during the 19th century proved that plant growth can be achieved from nutrient solutions in water without soil, with or without a medium. From those experiments, it was concluded that some elements are required in large amounts (macronutrients): nitrogen, phosphorus, potassium, sulphur, calcium, and magnesium. Further experiments helped identifying elements required in smaller amounts (micronutrients): iron, chlorine, manganese, boron, zinc, copper, and molybdenum (Resh, 2016).

Knowledge on plant nutrition remained within scientific research until the 1920s, when the greenhouse industry gained interest in hydroponics to reduce costs resulting from periodic soil replacement due to problems with its structure, fertility and pests. In the 1930s, Gericke coined the term hydroponics, from Greek hydro (water) and ponos (labour). The development of plastics and the implementation of automation technologies, helped the commercial development of hydroponics. Hydroponic culture in greenhouse conditions can have yields $25 \%$ higher than soil greenhouse culture, and 10 times higher than outdoors soil culture (Raviv and Lieth, 2007; Resh, 2016). For example, greenhouse tomato production reaches 50$80 \mathrm{~kg} \mathrm{~m}^{-2} \mathrm{yr}^{-1}$ with water use of $12-20 \mathrm{~L} \mathrm{~kg}^{-1}$ tomato, compared to field tomato production at 3-8 $\mathrm{kg} \mathrm{m}^{-2} \mathrm{yr}^{-1}$ with water use of $60 \mathrm{~L} \mathrm{~kg}^{-1}$ (Kelley et al., 2010; Nederhoff and Stanghellini, 2010).

Generally, a greenhouse hydroponic system consists of: 1) water source, 2) irrigation, and 3) mixing tank.

\footnotetext{
${ }^{1}$ Many hydroponic methods require some type of medium, so they can be referred to as 'soilless culture', whereas water-only culture would be true hydroponics. In this thesis, the term hydroponics is used interchangeably with the broad range of soilless plant production.
} 
Water source. Common sources include rainwater, surface water and ground water. The main consideration is salinity, measured as electrical conductivity (EC). Desirable EC is less than $0.5 \mathrm{mS} \mathrm{cm}^{-1}$ (Sonneveld and Voogt, 2009). Sand filtration is often used to achieve desired water quality. Desalination of sea water has been tested in research, but its cost is still very high for commercial implementation (Raviv and Lieth, 2007).

Irrigation. Multiple techniques are available to carry the nutrient solution to the roots of plants. Sprinklers apply irrigation from overhead installations, facilitating movement of vehicles and persons. Drip irrigation is the most common technique and it allows for uniform pressure distribution. Nutrient film technique (NFT) provides a thin layer of water continuously in contact with the roots without using substrate, it provides ease of control but it lacks a buffer and has a potential for spreading root-borne disease. Raft or deep water culture (DWC) operates as NFT with the added benefit of a large buffer to prevent nutrient and temperature fluctuations. Aeroponics supplies water to the roots of suspended plants by spraying. Ebb and flow systems irrigate by repeatedly flooding and draining container-grown plants. Small systems for grass, sprouts and microgreens consist of manually irrigated trays on shells or automatically irrigated rotating drums (Raviv and Lieth, 2007; Resh, 2016).

Mixing tank. Stock tanks supply controlled amounts of highly concentrated fertilizer into a mixing tank, where they are diluted with fresh water to a desired concentration. If recirculated, reuse water is also received in this tank. Commonly, EC is used to determine the levels of the desired nutrient mix, but some nutrients may accumulate over time and they need to be removed from the recirculating water using techniques like reverse osmosis (Raviv and Lieth, 2007).

Other processes in greenhouse horticulture include water filtration and disinfection, using processes like heating, UV radiation, and chemical treatment (Sonneveld and Voogt, 2009). 


\subsection{Mathematical models in aquaponics}

As mentioned in the previous section, the main challenge in aquaponics consists of balancing the needs of multiple organisms, present in the aquaculture and hydroponic loops. Experimental studies alone would be too costly and potentially harmful for the welfare of the aquatic species. Therefore, mathematical models are a suitable tool to improve the knowledge of aquaponics. Mathematical models provide a surrogate for the system they represent, useful when the system does not exist yet or when specific experiments on an existing system would be costly, dangerous, or ethically unacceptable (Birta and Arbez, 2013).

Scientific literature on mathematical models for aquaponic systems is limited, but it has grown in recent years. For example, Forchino et al. (2017) investigated the environmental impacts of two coupled aquaponic systems with different plant growing methods, using a model for Life Cycle Assessment (LCA). Goddek et al. (2016) and Karimanzira et al. (2016) performed model-based studies to investigate the dynamics of water quality and nutrients like $\mathrm{N}$ and $\mathrm{P}$, using system dynamics and mathematical models for system components available in literature. Cerozi and Fitzsimmons (2017) investigated balances of dissolved P in an aquaponic system, based on a model supported by experimental measurements. Goddek and Keesman (2018) performed a model-based study to investigate the potential of desalination to improve N utilization in decoupled aquaponic systems. Goddek and Körner (2019) developed a detailed model describing the different dynamics of the components and evaluating performance on yield, energy, $\mathrm{N}$ and $\mathrm{P}$ utilization on three scenarios for different locations in the world. Several of these studies discuss the need to evaluate detailed nutrient dynamics and uptake in future research.

In order to further improve aquaponic systems, it is necessary to improve the knowledge on the dynamics of all macronutrients ${ }^{1}$, which constitute the building blocks for fish and plants. Some nutrients, like $N$ and $P$, are often studied, because they are excreted in abundance by fish, and constitute an important potential for reuse as fertilizer for plants, However, other nutrients, like $\mathrm{Na}$ and $\mathrm{Ca}$, are rarely investigated in fish wastewater because they present no harm for fish, but they are potentially harmful for plants. Understanding the macronutrient dynamics in aquaponics is an essential step towards the identification of opportunities and bottlenecks in the exchange of water between fish and plants.

\footnotetext{
${ }^{1}$ The term 'macronutrient' often refers to the main food components: carbohydrates, protein and fat. In this thesis, the term is used to refer to the elements found in greater concentrations on both fish and plants: C, N, P, K, Ca, Mg, and Na.
} 
However, the complexity of aquaponic systems in particular, and all biological production systems in general, presents challenges for model development. Datadriven models (black box approach) can be easily calibrated, but they lack robustness. Complex physiological models (white box approach) can describe diverse system conditions, but they have a large number of parameters, and are thus difficult to calibrate. Therefore, it is often suggested to combine experimental measurements with mathematical models (grey box approach). Combining live in-situ measurements with mathematical models is the basis of precision farming (Føre et al., 2018), aimed at providing resources at the time and place required, leading to improved productivity and better animal welfare.

Additionally, biological systems often involve large uncertainties. It is therefore recommended to provide a transparent representation of these uncertainties on each level of a model (Uusitalo et al., 2015). However, as yet, limited attention has been given to quantify the uncertainties in aquaponic systems.

This thesis investigates water and nutrient balances and their uncertainties in decoupled aquaponic systems, through the development of mathematical models. It studies tomato and tilapia. Combining these products in a single loop is not ideal, due to significant differences between their requirements for water quality and environmental conditions. However, the knowledge from independently successful commercial systems, together with experimental data from literature and a demonstration site, provide a sound basis for the design and management of aquaponic systems. The models were based on the decoupled configuration proposed in the project INAPRO.

The mathematical models in this thesis were developed using the following approaches:

System dynamics. The models are described by a set of differential equations and supporting algebraic equations.

Semi-physical. Model development combines the mathematical description of general physical processes with experimental data (grey box).

Modularity. Each unit of the aquaponic system is represented as a module, with its own system of equations and corresponding solution.

System-level scale. The model description focuses on system behaviour, neglecting spatial variations and molecular transport phenomena.

Deterministic (with stochastic elements). The models predict single trajectories for each output, but they account for uncertainties.

Sequential. The results from one module are used as inputs for the next module in series (solved one-by-one). Feedback loops and their impacts are considered, but they are not used for the numerical solution. 
This thesis will by no means offer a complete solution for understanding the dynamics of nutrients in aquaponic systems, but it provides a few insights into the intricate processes involved in the technological application of the cycles present in nature.

In summary:

- Industrialized food production has led to centralization and dependencies, threatening food security, while externalizing environmental and social impacts.

- Aquaponics is an option for sustainable local food production.

- The main technical challenge in the development of commercial aquaponics consists in balancing different optimal requirements for multiple species.

- Decoupled aquaponic systems facilitate the operation of fish and plant loops independently, under their own optimal conditions.

- Aquaponic systems are complex and uncertain. Mathematical modelling and simulation combined with experimental measurements can help further developing this technology.

\subsection{Research objective}

As mentioned in the previous section, there is increasing research on mathematical models for aquaponic systems. However, there are limited studies regarding the dynamics of nutrients, especially accounting for uncertainties.

Therefore, the objective of this thesis was to investigate the dynamics of water and nutrient balances in aquaponics and their uncertainties, addressing the following research questions:

1. What are the opportunities, challenges, and possible solutions in closing cycles of water and nutrients in decoupled aquaponic systems?

2. What are the challenges and possible solutions in modelling nutrient balances and their uncertainties for aquaponics?

These questions guide the studies presented in the chapters that follow. 


\subsection{Contents of this thesis}

This thesis is divided in six chapters (Fig. 1.4). Chapters 1 and 6 provide a general introduction and general discussion. Chapters 2 through 5 consist of research studies.

Chapter 2. Model of an aquaponic system for minimised water, energy and nitrogen requirements. This chapter presents a model-based study coupling a RAS for tilapia with and an NFT hydroponic system for tomato. The simulation results show that fish can provide $26 \%$ of the $N$ requirements for plants. Furthermore, it is shown that variations in $\mathrm{NO}_{3}$ concentrations in the fish loop can be decreased by $35 \%$, using a water management strategy that sends water from fish to plants based on a schedule and amounts proportional to the fish feed.

Chapter 3. Model-based management strategy for resource efficient design and operation of an aquaponic system. This chapter presents a model-based study supported by a demonstration aquaponic system in Abtshagen, Germany. The model helped identifying imbalances in the system design. It predicted excess concentrations of total suspended solids (TSS) for fish, as well as excess $\mathrm{Na}, \mathrm{Ca}, \mathrm{Mg}$, and $\mathrm{NH}_{4}$ for plants. The model was used to develop an improved management strategy, preventing excess TSS, $\mathrm{Na}$, and $\mathrm{NH}_{4}$, decreasing fertilizer requirements, and achieving water efficiencies similar to commercial aquaculture and hydroponic systems.

Chapter 4. Set membership parameter estimation based on Voronoi vertices This chapter presents an algorithm proposed for the calibration of mathematical models. The algorithm uses a set-membership approach, which is a suitable alternative to statistical methods in the presence of limited and uncertain experimental data. The algorithm is based on a Voronoi tessellation, a novel method that characterises the feasible parameter set with (hyper)spheres, quantifying deviations from experimental data.

Chapter 5. Set-membership uncertainty assessment of a mathematical model for nutrient balances in fish growth and excretions. This chapter studied the dynamics and uncertainties in uptake and excretions of macronutrients during fish growth. A model was developed and calibrated using the Voronoi set-membership algorithm. Uncertainty propagation was assessed using Monte Carlo simulations. The model prediction explained $84 \%$ of the literature data variability for fish growth, $75 \%$ for digestive system content, and $28 \%$ for ammonia excretion rate. The uncertainty assessment helped identifying suitable experimental conditions for future research. 
Chapter 6. General discussion This chapter reflects on the research questions on decoupled aquaponic systems. The discussion is then extended to more general fields. First, suggesting a framework for modelling biological production systems under uncertainty. Finally, the socio-economic challenges for aquaponics and other methods for local food production are briefly discussed.

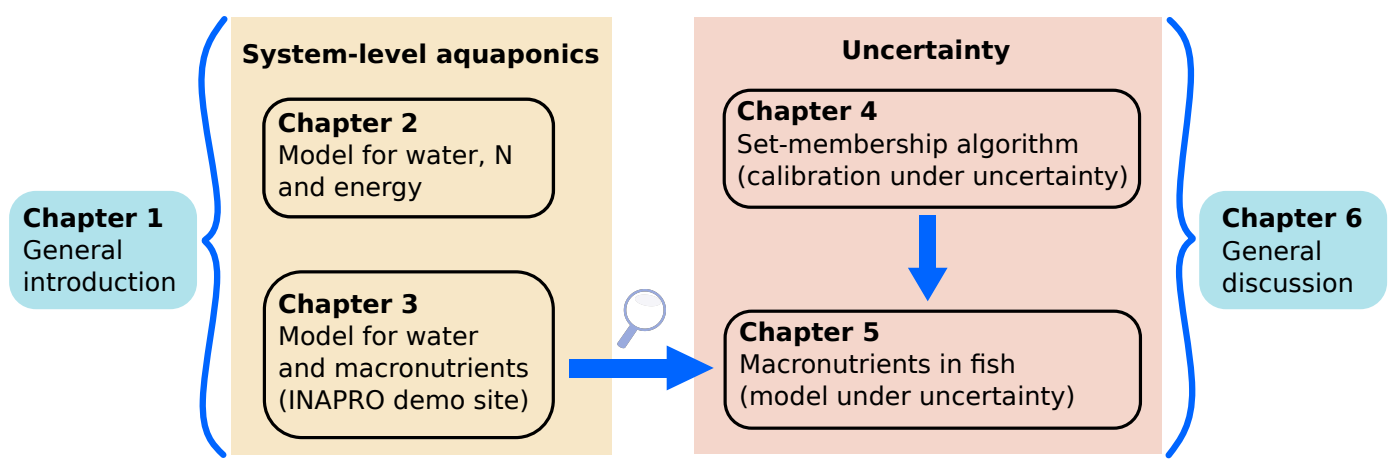

FIGURE 1.4: Contents of this thesis. 


\title{
Chapter 2
}

\section{Model of an aquaponic system for minimised water, energy and nitrogen requirements}

Published as:

Reyes Lastiri, D., Slinkert, T., Cappon, H. J., Baganz, D., Staaks, G., \& Keesman, K. J. (2016). Model of an aquaponic system for minimised water, energy and nitrogen requirements. In: Water Science and Technology, 74(1), 30-37.

DOI: https://doi .org/10.2166/wst.2016.127

\begin{abstract}
Water and nutrient savings can be established by coupling water streams between interacting processes. Wastewater from production processes contains nutrients like nitrogen, which can and should be recycled in order to meet future regulatory discharge demands.

Optimisation of interacting water systems is a complex task. An effective way of understanding, analysing and optimising such systems is by applying mathematical models.

The present modelling work aims at supporting the design of a nearly emissionfree aquaculture and hydroponic system (aquaponics), thus contributing to sustainable production and to food security for the XXI century.

Based on the model, a system that couples $40 \mathrm{~m}^{3}$ fish tanks and a hydroponic system of $1000 \mathrm{~m}^{2}$ can produce 5 ton of tilapia and 75 ton of tomato yearly. The system requires energy to condense and recover evaporated water, for lighting and heating, adding up to $1.3 \mathrm{GJ} \mathrm{m}^{-2}$ every year. In the suggested configuration, the fish can provide about $26 \%$ of the nitrogen required in a plant cycle. A coupling strategy that sends water from the fish to the plants in amounts proportional to the fish feed input, reduces the standard deviation of the $\mathrm{NO}_{3}^{-}$level in the fish cycle by $35 \%$.
\end{abstract}




\subsection{Introduction}

Maximising reuse of water and nutrients is needed to meet future regulatory discharge demands and minimise spillage. Moreover, water and nutrient recycling contributes to food security in the XXI century. In an aquaponic system, two food production systems are coupled: aquaculture (aquatic animal farming) and hydroponics (soilless plant farming). The nutrients excreted by the fish are used by the plants, which in turn clean the water that is recycled to the fish. In fact, this is a small scale example of a (closed) natural ecosystem with water and nutrient exchange.

Intensive fish production for commercial purposes can be accomplished in recirculating aquaculture systems (RAS), growing large amounts of fish with low water and space requirements. A RAS includes a water treatment facility to remove the waste products from the recirculated water. As a result of this purification step, some nutrients accumulate to concentrations that can be used by plants. In particular, ammonium $\left(\mathrm{NH}_{4}^{+}\right)$from the fish tanks is converted to nitrate $\left(\mathrm{NO}_{3}^{-}\right)$in a nitrification process. $\mathrm{NO}_{3}^{-}$has high value as fertilizer for plants, which can be grown without soil using the nutrient film technique (NFT) in a recirculating hydroponic system (RHS) (Rakocy et al., 2006; Bernstein, 2011).

Our case, known as the INAPRO system (INAPRO, 2014), combines tilapia and tomatoes in separate systems (farms) with two water cross-overs, i.e. the waste from the fish is used to fertilise the plants but is not directly circulated back to the fish. Instead, the water evaporated in the horticulture system is condensed and recovered without nutrients for the RAS system. This two decoupled recirculating systems can be individually optimised.

INAPRO combines the construction and operation of demonstration systems with the support of a model-based approach. The model presented herein describes water, energy and nitrogen balances. It aims at aiding in the sizing of system components and in the selection of operational strategies based on the dynamic behaviour of the system. For this study, the following aspects were evaluated: 1) NFT greenhouse area for tomato production and 2) RAS-RHS flowrate coupling strategy.

\subsection{Methods}

The model consists of a set of discretised ordinary differential equations (ODEs) assuming zero and first order dynamics of the production terms in the balances. The differential equations were solved numerically using the Euler Forward and fourth order Runge-Kutta methods. The model was implemented in Microsoft Excel ${ }^{\circledR}$, to facilitate updating the inputs and the direct visualisation of simulation results. 


\subsubsection{System description}

The INAPRO aquaponic system was modelled based on the components and configuration shown in Fig. 2.1: 1) Tilapia is grown in six fish tanks from the fingerling stage. 2) Solids are first removed in a mechanical drum filter. 3) Concentrated backwash water undergoes a secondary separation in a settling tank. 4) Filtrate from the drum filter is pumped to a biological filter for nitrification (conversion of $\mathrm{NH}_{4}^{+}$to $\mathrm{NO}_{3}^{-}$). 5) After disinfection and aeration, water is returned to the fish tanks. 6) A storage tank in a greenhouse for the hydroponic system receives the effluent of the settling tank and additional fertilizer when required. 7) Plants are grown in a NFT system in the greenhouse. 8) Water transpired by the plants in the greenhouse is recovered by condensation and sent back to the RAS.

The process units modelled were: fish tanks (FT), mechanical filter (MF), settling tank (ST), pump sump (PS), biofilter (BF), plant storage tank (PS) and the nutrient film technique channels (NFT). All units were modelled as perfectly mixed vessels. The pH levels were assumed controlled at 7.0 and 6.0 in the RAS and RHS, respectively. All units were modelled with a time-step of $1 \mathrm{hr}$. Fish and plant growth (FG and PG) were modelled with a time-step of 1 day.

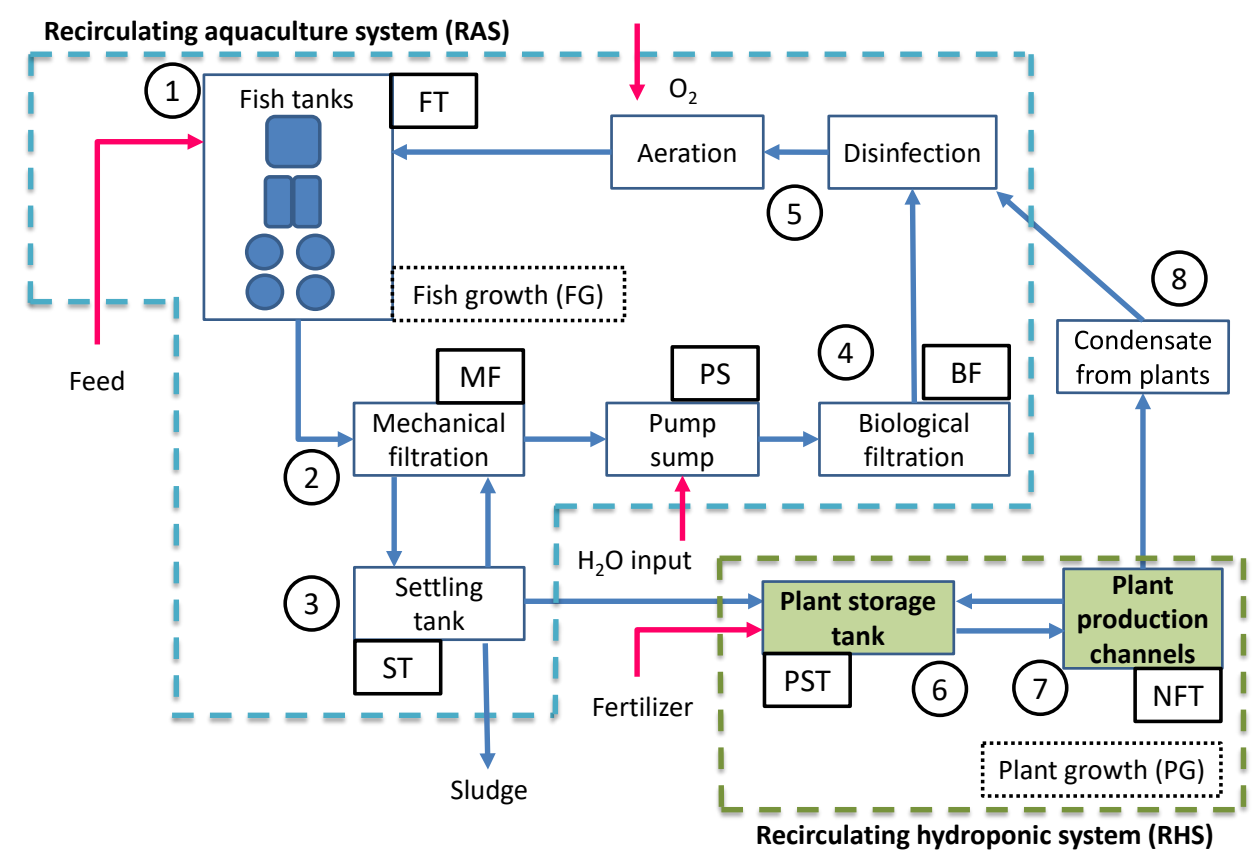

FIGURE 2.1: Main components of the INAPRO aquaponic system. 
This study aimed at assisting in the design and controlled coupling of the RAS and the RHS. Therefore, modelling detailed dynamics occurring within each unit was beyond its scope. Nonetheless, some phenomena that have been reported relevant in literature were included but simplified to zero and first order dynamics. The parameters listed in Table 2.1 are the result of these simplifications.

TABLE 2.1: Parameters used in the model of the aquaponic system

\begin{tabular}{|c|c|c|}
\hline Parameter & Description & Value (process unit) \\
\hline$\phi_{v, e v a}$ & Volumetric flow rate of water evaporation & $\begin{array}{l}1.0 l h r^{-1}(\mathrm{FT}) \\
0.5 l h r^{-1}(\mathrm{MF}, \mathrm{ST}, \mathrm{BF})\end{array}$ \\
\hline$k_{\text {vol }}$ & Constant rate of ammonia volatilisation & $0.16 h r^{-1}$ (FT, MF, ST, BF) \\
\hline$k_{a m m}$ & Constant rate of organic $\mathrm{N}$ ammonification & $0.15 h r^{-1}$ (FT, MF, ST, BF) \\
\hline$k_{\text {nit }}$ & Constant rate of ammonia nitrification & $0.10(\mathrm{BF})$ \\
\hline$k_{L U E}$ & Light use efficiency for plant growth & $4.0 \mathrm{gMJ}^{-1}(\mathrm{PG})$ \\
\hline$k_{L E C}$ & Plant overall light extinction coefficient & $0.7(\mathrm{PG})$ \\
\hline$k_{S L A}$ & Plant specific leaf area & $250 \mathrm{~cm}^{2} \mathrm{~g}^{-1}(\mathrm{PG})$ \\
\hline$k_{D M C}$ & Plant dry matter content & $\begin{array}{l}0.11 \text { (PG leaf); } \\
0.75 \text { (PG fruit) }\end{array}$ \\
\hline$k_{r b}$ & Plant leaf boundary resistance & $200 \mathrm{~s} \mathrm{~m}^{-1}$ (PG) \\
\hline
\end{tabular}

\subsubsection{Sub-models}

The model contains the following sub-models: 1) volumes and water flow rates, 2) nitrogen conversion processes, 3) fish growth and staggered production, 4) plant growth and nitrogen uptake, 5) plant evaporation, and 6) greenhouse climate. Assumptions and parameters used in each sub-model are provided in this section.

1) Volumes and water flow rates. The sizes of the RAS components were provided as a guideline by the INAPRO consortium: FT $=40 \mathrm{~m}^{3}, \mathrm{MF}=0.5 \mathrm{~m}^{3}\left(1 \mathrm{~m}^{2}\right)$, $\mathrm{ST}=10 \mathrm{~m}^{3}, \mathrm{BF}=0.5 \mathrm{~m}^{3}$ and PS $=10 \mathrm{~m}^{3}$. Water density $\left(\rho_{w}\right)$ was assumed constant. The amount of water in the pipes, and the transportation times between units were neglected. The pumps operate with a fixed flow rate. All units were modelled as reservoirs operating at constant volume:

$$
\rho_{w} \frac{d V}{d t}=0=\sum \phi_{v, \text { in }}-\sum \phi_{v, o u t}-\phi_{v, e v a}
$$

where water flow rates entering and leaving each unit are assigned based on Fig. 2.1, and $\phi_{v, e v a}$ is based on Table 2.1. 
The drum filter was assumed to operate at 70\% efficiency of solids removal (Timmons and Ebeling, 2010). Solids leave the filter as backwash flow to the secondary clarifier. The settling tank was modelled based on results obtained by Bergheim et al. (1998), represented as a two-layer vessel with each layer perfectly mixed, assuming settling efficiency of $60 \%$, with $5 \%$ re-suspension from the bottom layer due to turbulence. The effect of particle size in filtration processes was neglected.

2) Nitrogen conversion processes. $N$ conversion was modelled for fish tanks, mechanical filter, secondary clarifier, biological filter and NFT storage tank. $N$ is present as nitrate $\left(\mathrm{NO}_{3}^{-}\right)$, total ammonia nitrogen (TAN) constituted by ammonia $\left(\mathrm{NH}_{3}\right)$ and ammonium $\left(\mathrm{NH}_{4}^{+}\right)$, organic $\mathrm{N}$ in suspended solids, and organic $\mathrm{N}$ in settled solids. It was assumed that both the mechanical filter and the biological filter show a quasi-steady state behaviour, without $\mathrm{N}$ accumulation.

In the fish tanks, $N$ is supplied in the form of feed protein with $25 \% N$ (mass). $5 \%$ of the feed was assumed to be uneaten. Fish were assumed to accumulate $N$ with $32 \%$ efficiency, excrete $40 \%$ as $\mathrm{NH}_{3}$ and $28 \%$ in faeces (Rafiee and Saad, 2005). Nutrients in NFT channels were assumed to be in quasi-steady state.

The general balances for the $N$ forms in each of the units in the system are described by:

$$
\begin{aligned}
& \frac{d m_{N O 3}}{d t}=\sum \phi_{N O 3, \text { in }}-\sum \phi_{N O 3, o u t}+\phi_{n i t} \\
& \frac{d m_{T A N}}{d t}=\sum \phi_{T A N, \text { in }}-\sum \phi_{T A N, \text { out }}+\phi_{a m m}-\phi_{\text {nit }}-\phi_{v o l} \\
& \frac{d m_{N o r g}}{d t}=\sum \phi_{N o r g, \text { in }}-\sum \phi_{\text {Norg,out }}-\phi_{a m m}
\end{aligned}
$$

Ammonification $\left(\phi_{a m m}\right)$ and ammonia volatilisation $\left(\phi_{v o l}\right)$ were assumed to take place in all tanks except the pump sump. In the settling tank, ammonification was modelled in both layers (settled and suspended solids). Nitrification was assumed to take place only in the drum filter and in the biofilter after the previous two processes. The mass of gas ammonia follows from the equilibrium constant at the given $\mathrm{pH}$. The rate parameters for these processes are listed in Table 2.1.

$$
\begin{aligned}
\phi_{\text {vol }} & =k_{\text {vol }} \cdot m_{\text {TAN }} /\left(1+10^{9.25-p H}\right) \\
\phi_{\text {amm }} & =k_{\text {amm }} \cdot m_{\text {Norg }} \\
\phi_{\text {amm }} & =k_{\text {nit }} \cdot\left(\phi_{\text {TAN,in }}-\phi_{\text {vol }}\right)
\end{aligned}
$$


3) Fish growth and staggered production. Fish growth in each fish tank is described by the general mass balance:

$$
\frac{d m_{f s h}}{d t}=\phi_{f e d} \cdot F C R-m_{f s h} \cdot k_{f s h, m r t}-\phi_{h r v}
$$

where feeding rate $\left(\phi_{f e d}\right)$ and feed conversion ratio $(F C R)$ were obtained from experimental data in the ASTAF-PRO system from the Leibniz Institute of Freshwater Ecology and Inland Fisheries (IGB), fitted to polynomial curves based on the day of the current production cycle $\left(d_{c y c}\right)$ :

$$
\phi_{f e d}= \begin{cases}0.004 \cdot d_{c y c}+0.00996 & d_{c y c}<10 \\ 0.045 & 10 \leq d_{c y c}<24 \\ 0.571 \cdot\left(d_{c y c}+5.925\right)^{-0.757} & 24 \leq d_{c y c}<195 \\ 0.005 & 195 \leq d_{c y c}<211 \\ 0 & d_{c y c} \geq 211\end{cases}
$$

The length of each complete fish growth cycle was modelled to a fixed 241 days. Each batch of fish starts in the fingerling tank. At the beginning of month 3 , fish are moved to two raising tanks. At the beginning of month 8 , fish are moved to a starvation tank, where growth is completed at the end of month 8 . Fish stocking density was limited to a maximum of $84 \mathrm{~kg} \mathrm{~m}^{-3}$ with start and end weights of 10 and $650 \mathrm{~g}$, respectively.

The model assumed controlled temperature in the fish tanks, maintained at $28^{\circ} \mathrm{C}$, fish mortality rate $\left(k_{f s h, m r t}\right)$ equal to $10 \%$ per growth cycle, and harvest loss of $5 \%$.

4) Plant growth. The plant growth model was based on light use efficiency (LUE), adapted from TOMSIM (Heuvelink, 1996; Heuvelink, 1999). Nutrients and water were assumed readily available for the plants, i.e. plant growth is only determined by irradiation:

$$
\frac{d m_{p l t}}{d t}=k_{L U E} \cdot Q_{P A R, i n} \cdot\left(1-e^{k_{L E C} \cdot L A I}\right)
$$

Where $Q_{P A R, \text { in }}$ is the incoming photosynthetically active radiation, and $k_{L E C}$ is the light extinction assumed constant (Table 2.1).

Plant dry matter growth was partitioned into leaf growth $\left(m_{l f}\right)$ and fruit $\left(m_{f r}\right)$ growth, both modelled based on the leaf area index $(L A I)$. LAI was modelled assuming a constant specific leaf area $\left(k_{S L A}\right.$, Table 2.1). The value of $L A I$ was updated daily $\left(\Delta t_{p l t}=1\right.$ day $)$, and it was limited to a maximum of 3.0, assuming removal of 
old leaves:

$$
\begin{gathered}
\frac{d m_{l f}}{d t}= \begin{cases}0.5 \cdot \frac{d m_{p l t}}{d t} & L A I<2.5 \\
0.1 \cdot \frac{d m_{p l t}}{d t} & L A I \geq 2.5\end{cases} \\
\frac{d m_{f r}}{d t}= \begin{cases}0 . & L A I<2.5 \\
0.8 \cdot \frac{d m_{p l t}}{d t} & L A I \geq 2.5\end{cases} \\
\frac{d L A I}{d t}=k_{S L A} \cdot \frac{m_{l f, 1}-m_{l f, 0}}{\Delta t_{p l t}}
\end{gathered}
$$

Water uptake by plants $\left(\phi_{v, p l t}\right)$ accounts for evapotranspiration $\left(\phi_{v, e t}\right)$ and water partition into leaf and fruit. Conversion of water into dry mass by photosynthesis is neglected. Nitrogen uptake was modelled to depend only on dry mass production, assuming constant dry matter content $\left(k_{D M C}\right.$, Table 2.1) (Gallardo et al., 2009):

$$
\begin{aligned}
\phi_{v, p l t} & =\phi_{v, e t}+\frac{1}{k_{D M C, l f}} \cdot \frac{d m_{l f}}{d t}+\frac{1}{k_{D M C, f r}} \cdot \frac{d m_{f r}}{d t} \\
\phi_{N, p l t} & =0.05569 \cdot\left(\frac{d m_{p l t}}{d t}\right)^{0.9016}
\end{aligned}
$$

5) Plant evapotranspiration. Plant evapotranspiration was modelled based on Stanghellini (1987) and Prenger et al. (2002). It accounts for LAI, vapour deficit in the greenhouse $(V D)$, irradiance-dependent leaf stomatal resistance $\left(r_{s}\right)$, constant leaf boundary resistance $\left(k_{r b}\right.$, Table 2.1), temperature in the greenhouse $(T)$, net intercepted radiation $\left(Q_{R n}\right)$, and the ratio of latent to sensible heat for $1^{\circ} \mathrm{C}$ change $(\epsilon)$ :

$$
\begin{aligned}
\phi_{v, e t} & =\frac{2 L A I}{(1+\epsilon) k_{r b}+r_{s}} \cdot\left(V D+\frac{\epsilon k_{r b} Q_{R n}}{2 L A I 2450}\right) \\
r_{s} & =82 \cdot \frac{4.30+Q_{R n} /(2 L A I)}{0.54+Q_{R n} /(2 L A I)} \cdot\left[1+00023 \cdot(T-24.5)^{2}\right] \\
\epsilon & =0.7584 e^{0.0518 T} \\
V D & =2.5+0.004 Q_{P A R, i n} \\
Q_{R n} & =0.86\left(1-e^{0.7 Q_{P A R, i n} L A I}\right)
\end{aligned}
$$

6) Greenhouse climate. Greenhouse climate control was modelled to keep a set point temperature of $17.5^{\circ} \mathrm{C}$. It includes screens that close under limited solar radiation. The screens close to minimise vapour losses with radiation below $55 \mathrm{~W} \mathrm{~m}^{-2}$, and open at $70 \mathrm{~W} \mathrm{~m}^{-2}$. Additional artificial lighting of $100 \mathrm{~W} \mathrm{~m}^{-2}$ was included, operating with different schedules depending on the season (Hashimoto et al., 1993). 
Both the screens and the greenhouse cover material are assumed to be in a quasisteady state, (without energy accumulation). The simplified differential equations from the energy balance were solved analytically. The greenhouse climate model accounts for weather conditions: meteorological wind speed, global radiation, ambient temperature, air pressure, cloud cover and relative humidity. Weather data was obtained from the Royal Dutch Meteorological Institute (KNMI, 2014). For a detailed description of the energy balances, the reader is referred to Slinkert (2015).

\subsubsection{Optimised design}

Two design aspects were studied: 1) controlled coupling strategy between RAS and RHS (constant flow rate vs. fish feed dependent flow rate) and 2) NFT production size (area).

For the coupling strategy, the objective was to minimise the variations in the $N$ content of the water sent from the RAS to the RHS.

For the NFT area sizing, the following penalty function $(p)$ is minimised, consisting of RAS water discharge $\left(\phi_{v, \text { out }}\right)$, clean water use $\left(\phi_{v, i n}\right)$, and $N$ provided by fertilizer $\left(\phi_{N, i n}\right)$ with respect to $\mathrm{N}$ plant uptake:

$$
p=p_{1} \cdot \phi_{v, \text { out }}+p_{2} \cdot \phi_{v, \text { in }}+p_{3} \cdot \frac{\phi_{N, \text { in }}}{\phi_{N, p l t}}
$$

where $p_{1}, p_{2}$ and $p_{3}$ are arbitrary penalty factors that describe a weighted function. For this study, their values were set to 5.0, 1.0 and 10, respectively.

\subsection{Results and discussion}

Model results for the weather conditions of the Netherlands in the period 2008-2009 are presented. These results show the development of the system from start-up until it reaches an equilibrium stage.

The comparison between the two proposed RAS-RHS coupling strategies is shown in Fig. 2.2. The variation in the nitrate levels of the water flowing to the RHS are higher for a coupling based on constant flow rate. Therefore, a variable coupling strategy, such as this fish-feed based strategy, is preferred.

Results from the penalty function $(p)$, used for resource minimisation when sizing the NFT production area, are shown in Fig. 2.3. A minimum penalty is found at an NFT area of $1000 \mathrm{~m}^{2}$, which is also an area that matches the rainfall that can be captured to match the clean water usage. Different sizes of a buffer tank (BUF) used to accumulate water seasonally were simulated, but they have no impact on the water consumption and discharge. 

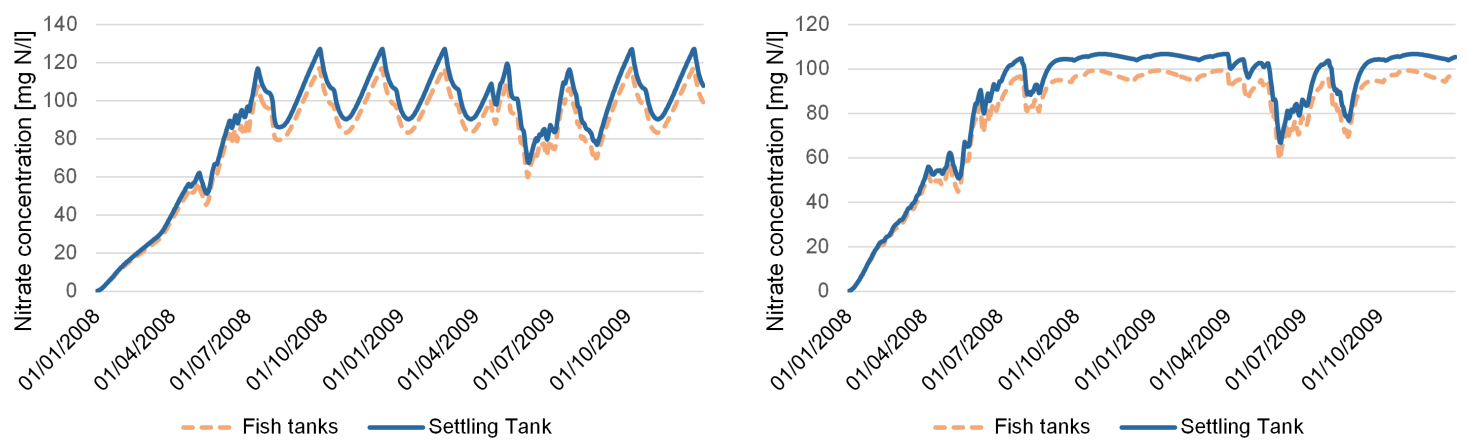

FIGURE 2.2: Nitrate concentration in FT and ST for constant flow coupling (left) and fish feed based coupling (right). The effluent of ST is supplied to the plants, which require a stable nutrient concentration. After system stabilization (month

8) the standard deviations are $13.23 \mathrm{mg} \mathrm{L}^{-1}$ (left), and $8.56 \mathrm{mg} \mathrm{L}^{-1}$ (right).
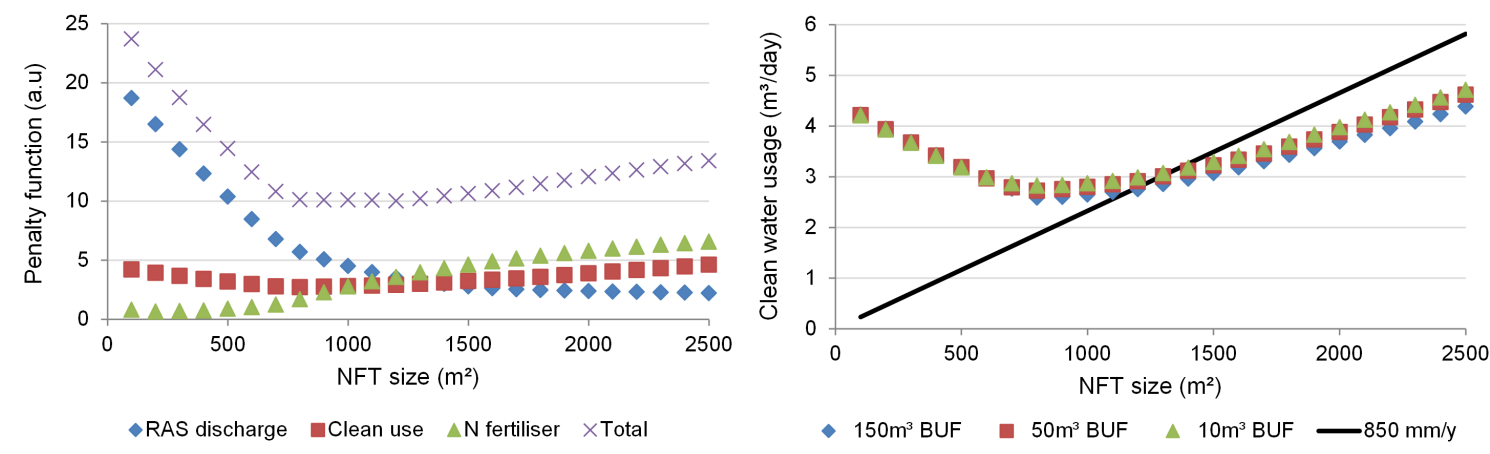

FIGURE 2.3: Minimisation of the penalty function $(p)$ with respect to NFT size (left). Minimisation of water use with respect to NFT size under different buffer tank (BUF) sizes. A minimum is obtained around $1000 \mathrm{~m}^{2}$ NFT area. Larger NFT areas result in an increased clean water and fertilizer use.

Based on these results, an NFT area of $1000 \mathrm{~m}^{2}$ and a fish feed based coupling were selected for subsequent simulations.

The growth pattern of the fish staggered production is shown in Fig. 2.4. It corresponds to a system with a regular time interval of 2.5 months between cycles, which results in a regular feeding rate and helps preventing large TAN peaks in the fish tanks. The seesaw pattern resulting from the staggered production explains the variations observed in the nitrate concentration when coupling RAS-RHS at a constant flowrate.

The production of tomato is directly related to the solar radiation intercepted by the plants. Dry mass production reaches a peak in summer but it is very limited in winter during the development of new plants (Fig. 2.5).

Greenhouse temperature obtained from the climate sub-model are shown in Fig. 2.6. During winter, the difference between the outside temperature and the set-point reaches about $25^{\circ} \mathrm{C}$. 


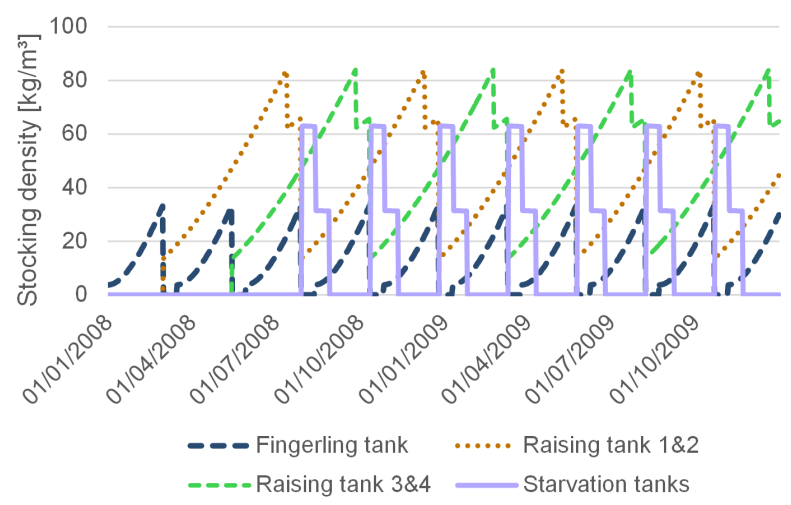

FIGURE 2.4: Fish stocking density with staggered production in the fish tanks and regular time interval of 2.5 months between cycles.
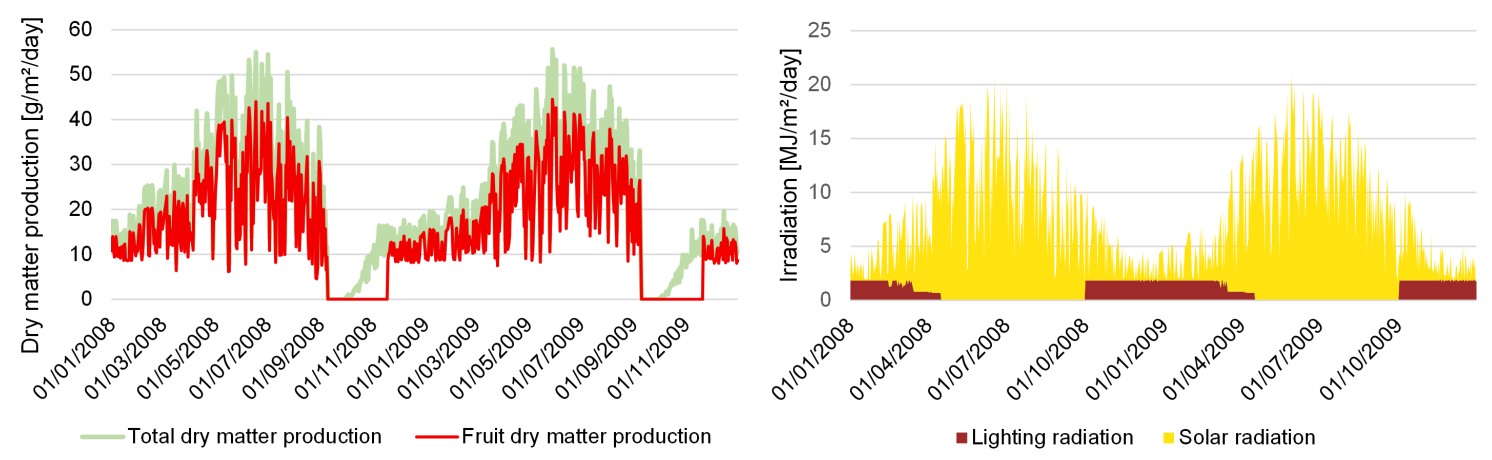

FIGURE 2.5: Dry mass production of plant and fruit (left). Natural and artificial radiation in the greenhouse (right). The strong dependence of plant growth on solar radiation is observed.
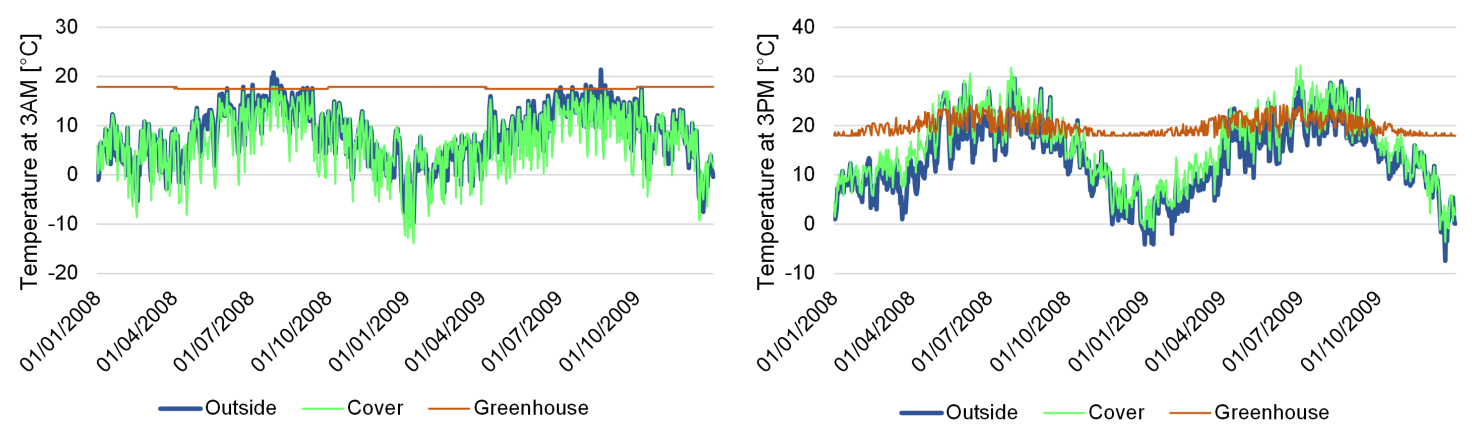

FIGURE 2.6: Greenhouse temperatures during the night (left) and day (right).

The monthly energy balance in the greenhouse is depicted in Fig. 2.7. The greenhouse requires secondary energy inputs to operate, such as heat and electricity. Due to the temperature differences during winter, a significant amount heat was esti-

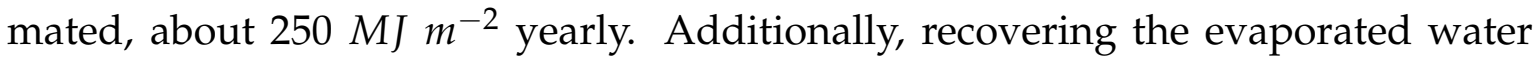
in the greenhouse by condensation for its recirculation in the RAS was estimated to consume yearly $740 \mathrm{MJ} \mathrm{m}$. Lighting, mainly during winter, was estimated to consume about $340 \mathrm{MJ} \mathrm{m} \mathrm{m}^{-2}$ per year. 


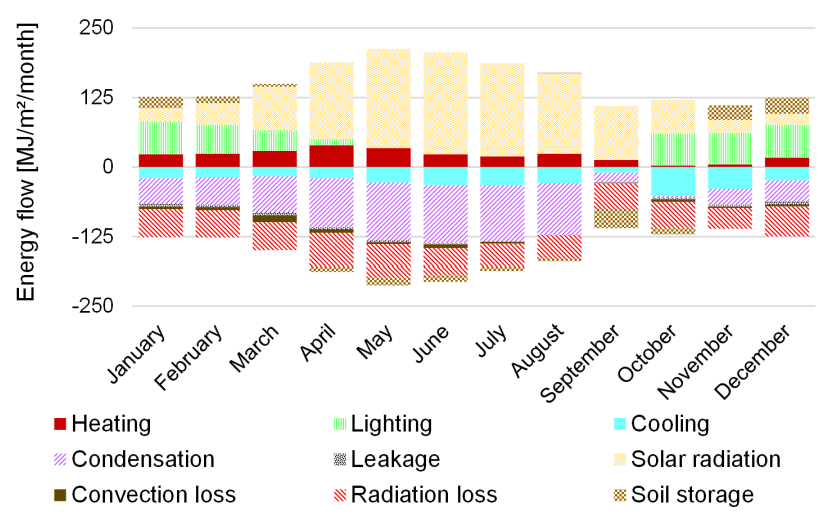

FIGURE 2.7: Monthly energy flows entering (positive values) and leaving (negative values) the greenhouse. The energy used for condensation cannot be supplied with solar radiation alone, and secondary energy sources are required.
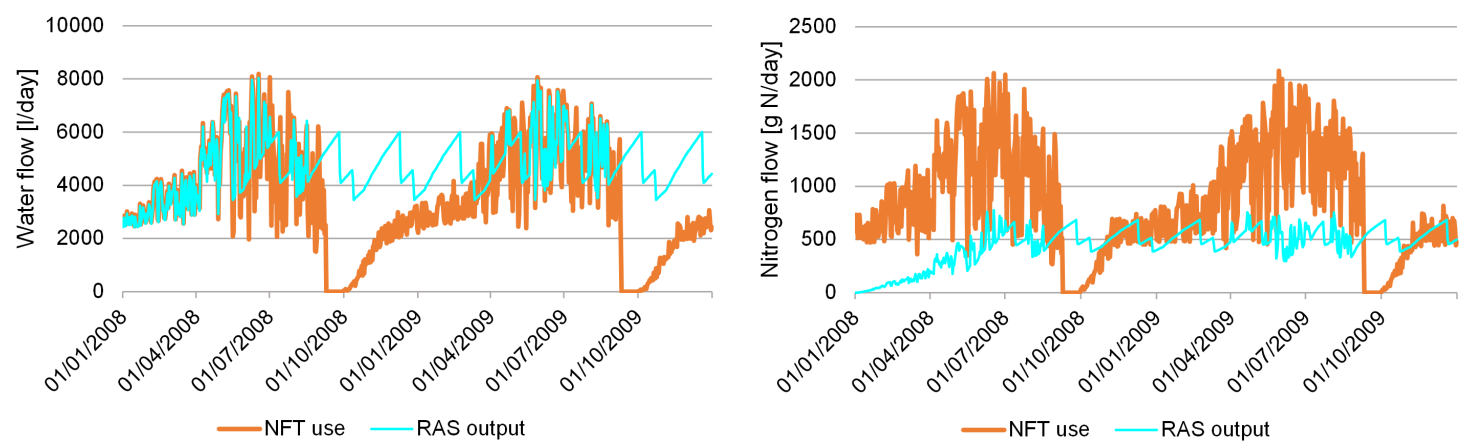

FIGURE 2.8: Water flow (left) and nitrogen flow (right) between the RAS and the NFT hydroponic system. RAS water output is not utilised by the plants between $01 / 10-01 / 04$ and it is discharged.

The resulting water and nitrogen use in the RAS-RHS coupling is shown in Fig. 2.8. During summer, the RAS system can deliver more than the minimum water requirement of the plants; the clean water that returns to the RAS dilutes the system and cools it down. However, during winter the plants are not developed enough to take the nutrient output of the RAS and about $530 \mathrm{~m}^{3} \mathrm{yr}^{-1}$ of water has to be discharged as sewage, in order to prevent nitrate intoxication of tilapia. Additional losses such as evaporation and sludge discharge from the filter add up to a total consumption of about $800 \mathrm{~m}^{3} \mathrm{yr}^{-1}$ of clean water to refill the system.

Estimated $\mathrm{N}$ output from the RAS is much lower than the uptake by the plants, and about $130 \mathrm{~kg}-\mathrm{N}$ are required per plant cycle, added as fertiliser, mainly during summer (about $171 \mathrm{~kg}^{-} \mathrm{N} \mathrm{yr}^{-1}$ ). The RAS output could supply up to $56 \%$ of the $\mathrm{N}$ requirement per plant cycle if it is not discharged; however, due to the discharge in winter, only $26 \%$ is covered.

With the resulting sizes and operational strategies, the yearly production of the system reaches about 75 ton of tomato and 5 ton of tilapia. 


\subsection{Conclusions}

A mathematical model of a decoupled aquaponic system for the production of tilapia and tomato, INAPRO, was developed using zero and first order dynamics.

The model was based on the water and nitrogen dynamics, the dominant nutrient in an aquaponic cycle.

For the selected operational scheme, a RAS of $40 \mathrm{~m}^{3}$ coupled with a NFT hydroponic system of $1000 \mathrm{~m}^{2}$ were estimated to achieve a yearly production of 5 ton of tilapia and 75 ton of tomato, requiring $171 \mathrm{~kg}-\mathrm{N}$ of fertiliser.

The preferred RAS-NFT coupling strategy is a fish-feed based water exchange, which can help accomplishing a stable nitrate concentration as required by the plant growth.

Additional energy utilisation is required by the proposed system configuration, particularly during winter, a total of $1.3 \mathrm{GJ} \mathrm{m}^{-2} \mathrm{yr}^{-1}$. Energy management could be improved by preventing excess input of solar radiation in summer which would in turn decrease water evaporation and consequently reduce energy consumption for condensation. This improvement should take into consideration the radiation requirement for plant growth in order to maintain the expected tomato yield.

Excess water from the RAS cannot be utilised at all times by the plants, and it is discharged as sewage. The total water losses require $800 \mathrm{~m}^{3}$ of top-up clean water per year.

The aquaculture and the hydroponic systems were optimised individually, maximising their yields per water usage. However, fertiliser is still added. $26 \%$ of the $N$ required by a plant cycle can be covered by the RAS output, using the given water buffer capacities.

For future work, the dynamics of other nutrients and variables should be included, such as phosphorus $(P)$, sodium $(\mathrm{Na})$, magnesium $(\mathrm{Mg})$, dissolved oxygen (DO) and $\mathrm{pH}$. This will allow for the identification of further improvements in the system and the chance to include additional species to better utilise the resources available, while trying to reduce the need for fertiliser. 


\title{
Chapter 3
}

\section{Model-based management strategy for resource efficient design and operation of an aquaponic system}

Published as:

Reyes Lastiri, D., Geelen, C., Cappon, H. J., Rijnaarts, H. H., Baganz, D., Kloas, W., Karimanzira, D. \& Keesman, K. J. (2018). Model-based management strategy for resource efficient design and operation of an aquaponic system. Aquacultural engineering, 83, 27-39. DOI: https://doi .org/10.1016/j . aquaeng. 2018.07.001

\begin{abstract}
Aquaponics combines aquaculture with hydroponics, i.e. growing aquatic species and soilles plants in a single system. Commercial aquaponics is still in development. The main challenge consists in balancing the conditions required for the growth of multiple species, leading to a dynamic system with high complexity. Mathematical models improve our understanding of the complex dynamics in aquaponics, and thus support the development of efficient systems.

We investigated a water and nutrient management strategy for the efficient production of Nile tilapia (Oreochromis niloticus) and tomato (Solanum lycopersicum) in an existing INAPRO aquaponic demonstration system in Abtshagen, Germany. For this purpose, we developed a system-level mathematical model.

In our simulations, we found that the existing configuration and water management of the Abtshagen aquaponic system results in an excessive amount of water discharged from the aquaculture loop. Therefore, sending more nutrient-rich water from fish to plants can help reducing water and fertilizer consumption. However, this water transfer may lead to excess concentrations of some nutrients, which could stress fish, plants or both. Our simulations predicted excess concentrations of total suspended solids (TSS) for fish, and excess sodium $\left(\mathrm{Na}^{+}\right)$and ammonium nitrogen
\end{abstract}


$\left(\mathrm{NH}_{4}^{+}-\mathrm{N}\right)$ for plants. Furthermore, excess calcium $\left(\mathrm{Ca}^{2+}\right)$ and magnesium $\left(\mathrm{Mg}^{2+}\right)$ for plants was predicted, due to the use of local fresh water with relatively high concentrations of those ions.

We developed an improved management strategy to better balance resource efficiency and water quality. This management strategy prevents excess levels of TSS for fish, and $\mathrm{Na}^{+}$and $\mathrm{NH}_{4}^{+}-\mathrm{N}$ for plants. Under this strategy, simulated water requirements (263 $\mathrm{L} \mathrm{kg}^{-1}$ fish and $22 \mathrm{~L} \mathrm{~kg}^{-1}$ tomato) were similar to current commercial aquaculture and greenhouse horticulture. Simulated plant fertilizer requirements for $\mathrm{N}, \mathrm{Ca}$ and $\mathrm{Mg}$ (52, 46 and $9 \mathrm{mg} \mathrm{kg}^{-1}$ tomato, respectively) were one order of magnitude lower than in high efficient commercial closed greenhouse production.

\subsection{Introduction}

Land-based aquaculture can help increasing local fish production with low water consumption. Intensive recirculating aquaculture systems in the Netherlands can use less than $1 \mathrm{~m}^{3}$ water per $\mathrm{kg}$ fish, competing with livestock levels. Recirculating aquaculture production is increasing worldwide, but operational requirements like energy and water purification are still a challenge, resulting in high investment costs (FAO, 2021). High cost for water purification is due in part to the accumulation of excess nutrients. Hydroponic horticulture has been suggested and tested to utilise excess nutrients from aquaculture (Lewis et al., 1978). In the Netherlands, greenhouse horticulture is very well developed, but it depends on external fertilizer. Therefore, the current technological status of aquaculture and greenhouse horticulture presents an opportunity to balance their mutual needs.

Aquaponics is a production method that combines aquaculture with hydroponics. This combination has been long known: as rice fields combined with fish culture in South-East Asia (Coche, 1967), and as chinampas by the Aztecs (Turcios and Papenbrock, 2014). Researchers of recirculating aquaculture introduced the modern concept of aquaponics in the mid-1970s as a combination of intensive production systems (with low land and water use); but commercially competitive aquaponics is yet to be achieved (Love et al., 2015). The main challenge lies in balancing the water quality and the nutrients required by three or more biological systems (fish, plants and biodegradation bacteria) (Suhl et al., 2016). There is growing research based on mathematical models aimed at understanding the dynamics of water and nutrients in aquaponics for improved production. However, due to the large number of variables involved in a system-level analysis of aquaponics, each study has limitations. 
For example, Reyes Lastiri et al. (2016) presented a dynamic model for water and $N$ in aquaponics, showing that it is theoretically possible to achieve stable nitrate levels for fish, by sending water from fish to plants based on the fish feeding schedule. Karimanzira et al. (2016) developed a detailed dynamic model for water, $N$ and $P$ in a decoupled aquaponic system. Goddek et al. (2016) developed an agent-based model showing that it is theoretically possible to reduce nutrient consumption based on system-level sizing and design. However, these studies only describe balances for limited nutrients, configurations and management strategies.

Fertilizer and water consumption can be reduced by controlling the amount and frequency of water sent from fish to plants. But this water transfer can lead to accumulation of some nutrients to stressful levels for fish, plants or both. Therefore there is a trade-off between water transfer from fish to plants, and excessive nutrient concentrations that may result.

To balance the trade-off, one approach could be the introduction of additional (novel) technologies into aquaponics. For example, Goddek and Keesman (2018) proposed desalination to treat excess nitrate, but desalination units can be energy intensive and limited to large-scale applications. An alternative approach consists of evaluating multiple management strategies, and then selecting the most adequate. This alternative approach may not reach maximum resource recovery, but it does not introduce additional costs and it may help improving the productivity of an existing aquaponic system.

We propose a strategy for system design and management aimed at achieving a balance between reduced resource requirements and water quality conditions. For this purpose, we developed a mathematical model describing the system-level dynamics of water and multiple relevant nutrients in an existing aquaponic system.

\subsection{Methods}

The decoupled aquaponic system consists of two separate production loops: a recirculating aquaculture system (RAS) and a hydroponic system (HS).

\subsubsection{Description of the aquaponic system}

We studied one of the demonstration systems built and operated for semi-commercial and research purposes as part of INAPRO, a European Union (EU) project aimed at further developing aquaponics towards a commercial scale (INAPRO, 2014). The system design is based the ASTAF-PRO configuration (Kloas et al., 2015), and it is located in Abtshagen, Mecklenburg-West Pomerania, Germany. It consists a fish farm with $7.2 \mathrm{~m}^{3}$ of total fish tanks volume in a RAS area of $43 \mathrm{~m}^{2}$, and a Venlo type 
greenhouse of $139 \mathrm{~m}^{2}$ for hydroponic cultivation in nutrient film technique (NFT) channels with a net cultivated area of $62.6 \mathrm{~m}^{2}$. We studied the production of Nile tilapia (Oreochromis niloticus) and tomatoes (Solanum lycopersicum cv. Pureza).

Inputs to the system were young tilapia, young plants, water and fertilizer. Outputs from the system were market-size tilapia, tomatoes, sludge, wastewater and plant residue.

RAS and HS were operated as separate loops. Water was transferred from RAS to HS to provide some of the nutrients required by the plants. RAS and HS loops are described below and illustrated in Fig. 3.1. Component sizes are listed in Table 3.1.

RAS. Fish grew in 4 fish tanks (FTs) with circulating water. Water effluent from the FTs was directed to a settling tank (ST) to remove solids. Clean water from the ST was taken to a nitrification unit consisting of a pump sump (PS), a biofilter (BF), and a small tank catching filtered water (FW). Water from the filtered water tank was circulated back to the FTs. Settled sludge from the ST was removed periodically and directed to a post-purge unit (PP), consisting of a 3-chamber pit outside the building for secondary removal of solids by sedimentation. Clean water is added to the system from a fresh water tank (WT).

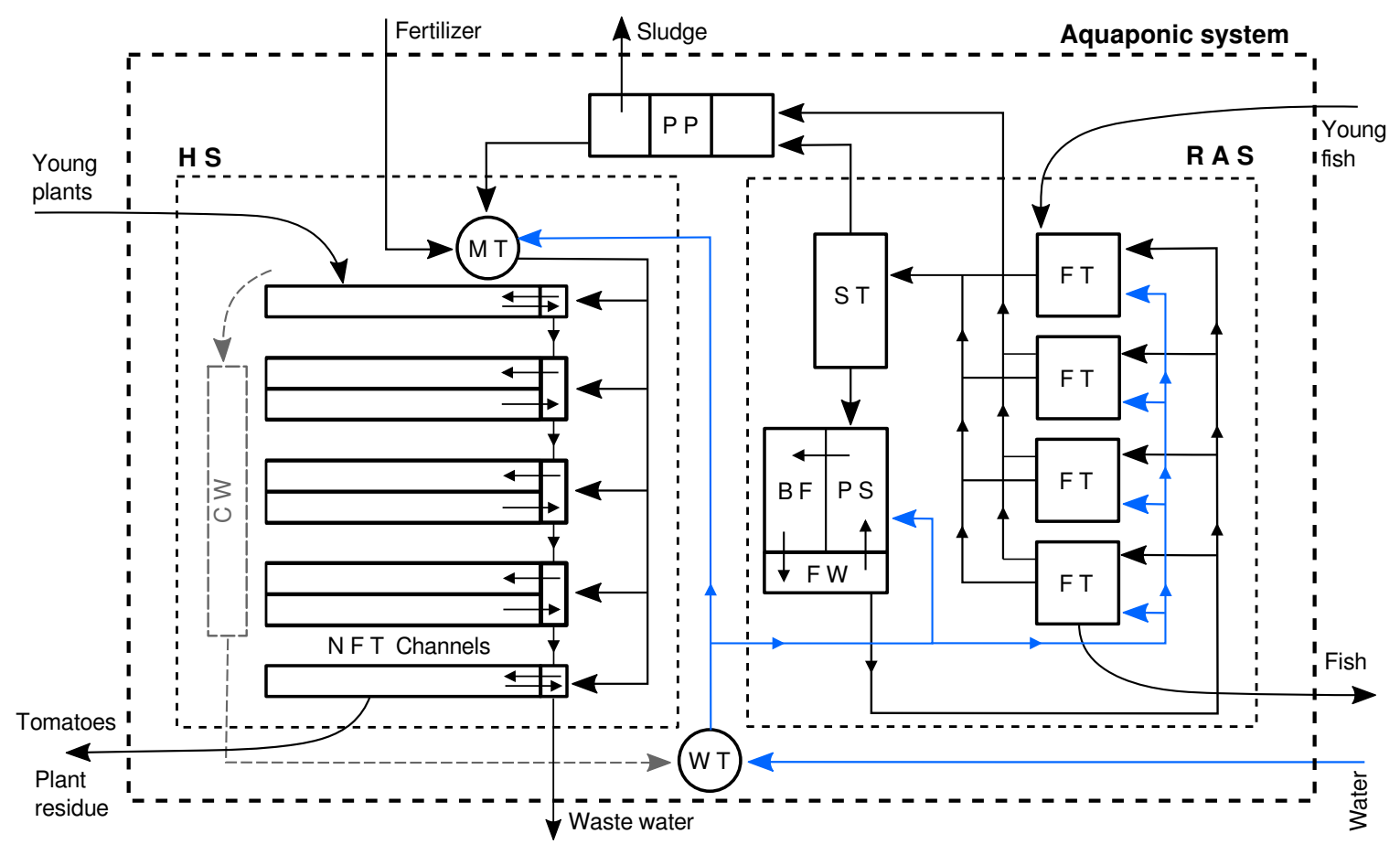

FIGURE 3.1: INAPRO demonstration aquaponic system in Abtshagen, Germany.

Dashed rectangles represent the boundaries of the recirculating aquaculture system (RAS), hydroponic system (HS), and overall aquaponic system.

Components are detailed in Table 3.1. 
TABLE 3.1: Components of the aquaponic system.

\begin{tabular}{lrl}
\hline Component & \multicolumn{1}{c}{ Size } & Comments \\
\hline Fish tanks (FT) & $4 \times 1.84 \mathrm{~m}^{3}$ & with 175 fish $/$ tank \\
Settling tank (ST) & $1.32 \mathrm{~m}^{3}$ & - \\
Pump sump (PS) & $2.38 \mathrm{~m}^{3}$ & - \\
Biofilter (BF) & $2.29 \mathrm{~m}^{3}$ & with $445 \mathrm{~m}^{2} / \mathrm{m}^{3}$ \\
Filtered water tank (FW) & $0.02 \mathrm{~m}^{3}$ & negligible volume, not modelled \\
Post-purge (PP) & $9.0 \mathrm{~m}^{3}$ & 3-chamber pit \\
Mixing tank (MT) & $1.1 \mathrm{~m}^{3}$ & - \\
Nutrient film technique (NFT) & $8 \times 10 \mathrm{~m}$ & with 2.4 plants $/ \mathrm{m}$ \\
Fresh water tank (WT) & $2 \mathrm{~m}^{3}$ & constant concentrations assumed \\
\hline
\end{tabular}

HS. Water with dissolved nutrients was received from the RAS after separation of solids at the PP. This water was stored inside the greenhouse in a mixing tank (MT). If needed, fertilizer was added to the MT to match minimum concentrations required by plants. The nutrient solution from the MT was sent to 5 small tanks that supply a set of NFT channels ( 3 double and 2 single). The single NFT channels were located near the greenhouse walls to maintain similar shading conditions in the double channels (condition required for other experimental studies). At the end of a plant production cycle, remaining water with low nutrient concentration was discarded. The aquaponic system includes a plan to recirculate condensed water from the HS back to the RAS loop (CW). However, this process was not yet available at the time of this study and therefore was not included in the model.

\subsubsection{Description of the mathematical model}

The mathematical model was developed following a dynamic, deterministic and modular approach, and based on algebraic and ordinary differential equations. Each module represents a component of the aquaponic system. The modules were modelled and calibrated based on data available from literature and gathered at the INAPRO demonstration site. Model and calibration routines were programmed in Python 3.4.

We modelled production of Nile tilapia in the RAS coupled with tomato in the HS. Design, configuration and flows between units were based on the operation of the demonstration system in Abtshagen.

Each module comprises mass balances for 3 state variables: 1) water volume $(V)$, soluble components (nutrients) $\left(m_{S}\right)$, and total suspended solids $\left(m_{T S S}\right)$. The state variables constitute model outputs $(y)$. The state variable $m_{S}$ consists in turn of macronutrients considered relevant for plant hydroponic cultivation: $\mathrm{NO}_{3}^{-}-\mathrm{N}$, TAN, $\mathrm{PO}_{4}^{3-}-\mathrm{P}, \mathrm{K}^{+}, \mathrm{Ca}^{2+}, \mathrm{Mg}^{2+}$, and $\mathrm{Na}^{+}$. We abbreviated these components as: $\mathrm{NO}_{3}, \mathrm{TAN}, \mathrm{P}, \mathrm{K}, \mathrm{Ca}, \mathrm{Mg}$, and $\mathrm{Na}$ 
Mass balances for each module were expressed in terms of inflows $\left(\phi_{i n}\right)$ and outflows $\left(\phi_{\text {out }}\right)($ Eqn. 3.1). Concentrations at effluents were calculated from the resulting states of each unit $C=m / V$. The simulation time step $(\Delta t)$ was $15 \mathrm{~min}$.

$$
\frac{d y}{d t}=\phi_{\text {in }}-\phi_{\text {out }}
$$

Mass balances for each module (Table A3.1), auxiliary equations (Table A3.2), and parameter values (Tables A3.3-A3.4) are provided in the Appendix of this chapter.

The filtered water tank (FW) has a negligible volume, therefore it was not modelled. The fresh water tank (WT) was only modelled as a supply with constant concentrations.

Assumptions for the system-level model were:

- Negligible volume of pipes.

- Constant density of water $\rho=1000 \mathrm{~kg} \mathrm{~m}^{-3}$ (neglecting effect of soluble and suspended matter).

- Chemical reactions only take place in the BF.

- Constant fresh water composition, based on measurements by REWA (2017).

- Ideal fish and plant growth, based on expert management from successful commercial operation in the region for tilapia aquaculture and tomato horticulture.

\section{Fish tank (FT)}

Fish was grown and harvested in FTs. Recirculating water enters at rate $\phi_{v, i n}$. Ideally, a FT should be fully refreshed twice per hour. Outflow occurred by overflow on a standing pipe at rate $\phi_{v, \text { out }}$. To start production, fish was added to the 4 tanks in a staggered sequence spaced by 45 days. Each tank was harvested once every 180 days alternating between tanks, providing 1 harvest every 45 days. At harvest, a tank was emptied at rate $\phi_{v, h r v}$ in $1 \Delta t$ (to the PP). During the same $\Delta t$, flow through the FT stopped $\left(\phi_{v, \text { in }}=\phi_{v, \text { out }}=0 \mathrm{~m}^{3} \mathrm{hr}^{-1}\right)$, and fresh water refills the FT at rate $\phi_{v, f r s}=V_{F T} / \Delta t$. After harvest, flow through the FT resumed and an additional FT volume of fresh water was added through a period of 2 days to clean the FT $\left(\phi_{v, f r s}=V_{F T} / 2\right.$ day $)$. Since the additional water in the 2 day period stays relatively clean, it was kept in the RAS loop. New fish was then added to the harvested tank. Growth conditions are reported in Suhl et al. (2016). 


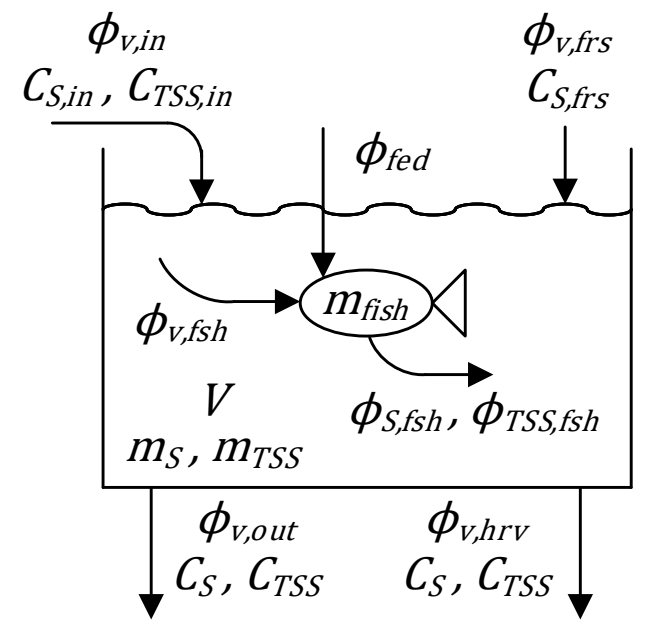

FIGURE 3.2: Model of the fish tank (FT).

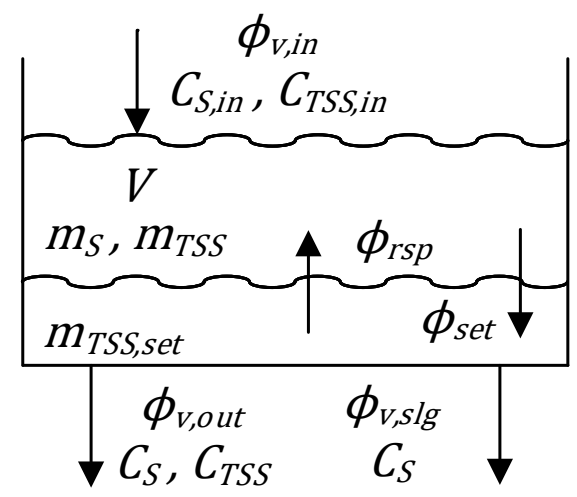

FIGURE 3.3: Model of the settling tank (ST).

Each FT was modelled as an ideally mixed tank. Fish growth and nutrient uptake was included as a sub-model of the FTs. Total fish in one tank was modelled as a single mass $m_{f s h}$ assuming ideal growth, based on a least-squares, second order polynomial regression from data of average growth per fish estimated by Autosoft (Jancke, 2016) (see Table A3.2 in the Appendix of this chapter). Fish constitutes up to $7 \%$ of the water volume in the FT and was therefore neglected in the calculation of $V$. It was assumed that all fish feed is consumed.

\section{Settling tank (ST)}

The ST was used to remove solids. Recirculating water entered at rate $\phi_{v, \text { in }}$ and left by overflow at rate $\phi_{v, o u t}$. Sludge was removed at rate $\phi_{v, s l g}$ once every time interval $\Delta_{t, \text { clean }}$ in 1 single time-step. TSS settled at a rate $\phi_{\text {set }}$, and undergo resuspension during sludge removal at a rate $\phi_{r s p}$. Therefore, an additional mass balance for settled solids was included in the model $\left(T_{S S_{\text {set }}}\right)$.

For soluble components $(S)$, the ST was modelled as an ideally mixed tank. For TSS, settling and resuspension rates were assumed linearly related to the mass of TSS in the water and sludge respectively.

\section{Pump sump (PS)}

The pump sump was used to balance water deficit and to pump water through the system. Recirculating water entered at rate $\phi_{v, \text { in }}$ and it was pumped up at constant rate $\phi_{v, \text { out }}$ (to the $\mathrm{BF}$ ). Water also entered from the filtered water tank of the biofilter at rate $\phi_{v, f l t}$. When inflows were not sufficient to keep the PS full, fresh water was added at rate $\phi_{v, \text { frs }}$ (from the WT).

The PS was modelled as an ideally mixed tank. 


\section{Biofilter (BF)}

The $\mathrm{BF}$ consisted of a trickling filter used for nitrification, i.e. conversion of ammonia $\left(\mathrm{NH}_{4}^{+}\right)$, which is toxic for the fish, into less toxic nitrate $\left(\mathrm{NO}_{3}^{-}\right)$. Recirculating water enters at a rate $\phi_{v, \text { in }}$ and leaves at a rate $\phi_{v, \text { out }}$. Biofiltered water is received in the FW tank (of negligible volume). Part of the effluent goes back to the PS $\left(\phi_{v, o u t, P S}\right)$ and the rest is circulated back to the FTs $\left(\phi_{v, \text { out }}\right)$.

The BF was modelled as a continuously stirred tank reactor (CSTR). Only $2.4 \%$ of the TAN mass is converted into bacteria biomass (Timmons and Ebeling, 2010), and this loss of $N$ was thus neglected. The simplified chemical reaction modelled was:

$$
\mathrm{NH}_{4}^{+}+2 \mathrm{O}_{2}+2 \mathrm{HCO}_{3}^{-} \rightarrow \mathrm{NO}_{3}^{-}+3 \mathrm{H}_{2} \mathrm{O}+2 \mathrm{CO}_{2}
$$

The reaction rate of TAN was assumed linear. It was assumed that water and TSS do not change through the BF, i.e. $d V / d t=0$ and $d m_{T S S} / d t=0$. It was also assumed that the $\mathrm{BF}$ is well aerated, therefore $\mathrm{O}_{2}$ does not become a limiting factor in the reaction, and $\mathrm{CO}_{2}$ leaves as it is produced.

Nitrification consumes alkalinity and results in a $\mathrm{pH}$ decrease. To correct this, $\mathrm{pH}$ buffers like $\mathrm{NaHCO}_{3}$ are commonly added in RAS before circulating water back to the fish. However, the system in Abtshagen showed no decrease in $\mathrm{pH}$ after nitrification. Therefore, addition of a pH buffer was not included in the model.

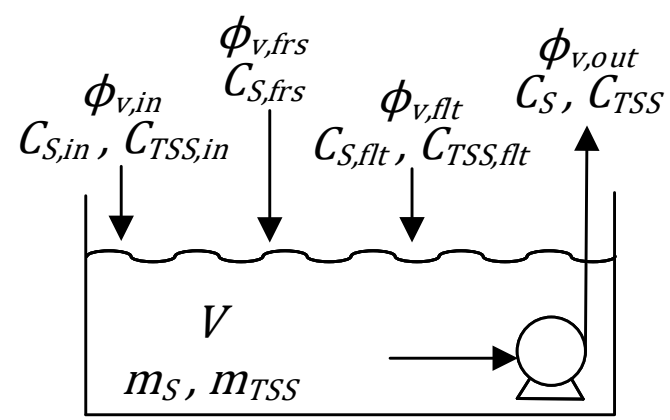

FIGURE 3.4: Model of the pump sump (PS).

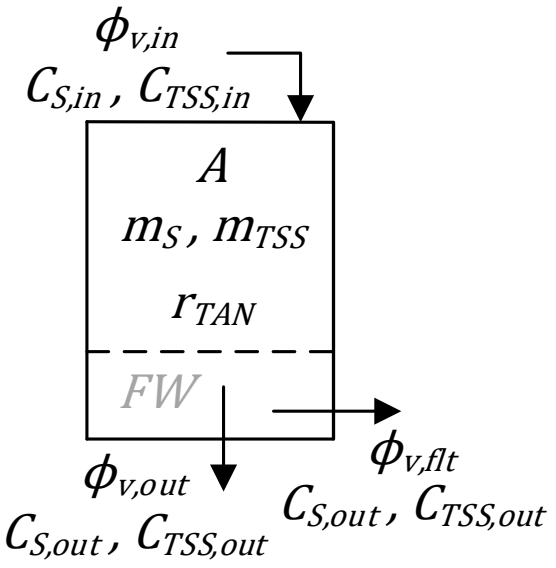

FIGURE 3.5: Model of the nitrification biofilter (BF). 


\section{Post purge (PP)}

The PP was used for secondary clarification by settling of solids and consists of a 3chamber pit. It operated as the connection unit between RAS and HS. Sludge from the ST entered at rate $\phi_{v, s l g}$. Water from the FTs emptied for harvest entered at rate $\phi_{v, h r v}$. Water was removed on demand to the HS at a rate $\phi_{v, \text { out }}$. When HS demand is low, water in the PP accumulates; if full, excess water was discharged at a rate $\phi_{v, \text { dis. }}$.

The 3 chambers were modelled as a single unit similar to the ST: an ideally mixed tank for $S$, and settling for TSS at a rate linearly related to its concentration.

\section{Mixing tank (MT)}

The MT received water from the RAS as well as fertilizer to match concentrations required by plants $\left(C_{S, \text { req }}\right)$. RAS water entered at rate $\phi_{v \text {, in }}$ and left after fertilizer addition at rate $\phi_{v, \text { out }}$. When $V$ dropped below $55 \%$, fresh water was added at rate $\phi_{v, f r s}$ to refill the MT in one $\Delta t$. Fertilizer was added at rate $\phi_{f r t}$ in 1 time-step $\Delta t$, based on the difference between required and present nutrient concentrations at the NFT tanks.

The MT was modelled as an ideally mixed tank. Fertilizer volume constitutes less than $0.1 \%$ of the flow rate leaving the MT. Therefore, $V$ increase due to fertilizer addition was neglected.

It was assumed that nutrients were added in their soluble ionic form (in practice, salts are added), neglecting the presence of other elements. It was also assumed that $\mathrm{N}$ was only added as $\mathrm{NO}_{3}^{-}$and no $\mathrm{Na}^{+}$was added.

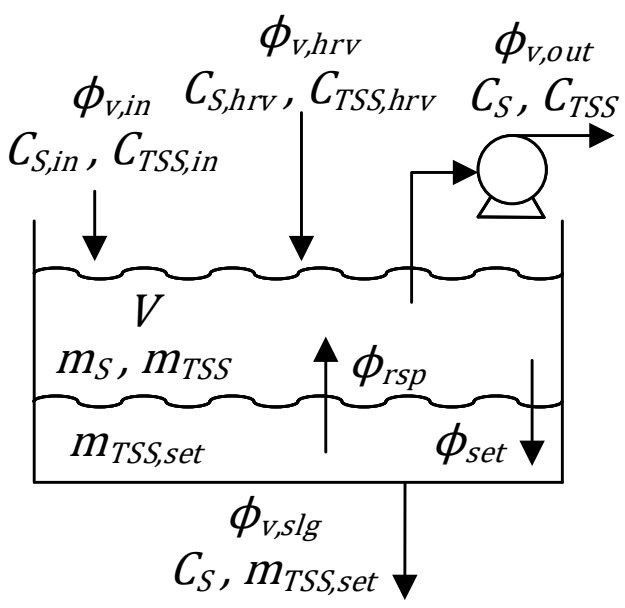

FIGURE 3.6: Model of the post purge tank (PP), consisting of a 3-chamber pit modelled as a single tank.

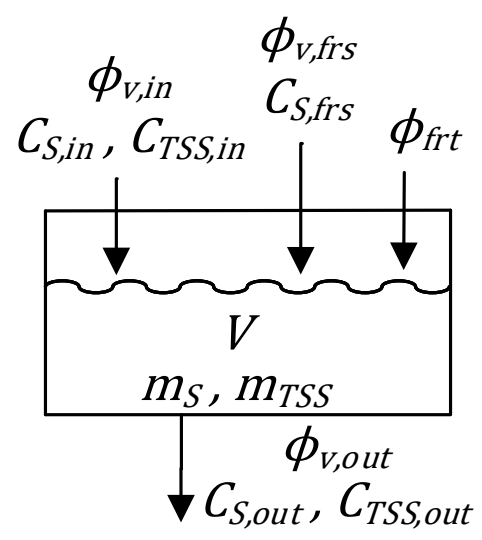

FIGURE 3.7: Model of the mixing tank (MT). 


\section{Nutrient film technique (NFT) unit}

The NFT unit consisted of small tanks that store water recirculated in channels where plants were grown. Water entered the NFT tanks at rate $\phi_{v, i n}$. Plants take up water at rate $\phi_{v, p l n}$ in the channels. After a yearly production cycle, all remaining water was discharged from the NFT tanks at rate $\phi_{v, \text { dis }}$ in $1 \Delta t$. Growth conditions in the greenhouse are reported in Suhl et al. (2016).

The group of NFT tanks and channels were modelled as a single ideally mixed tank. Changes in concentrations through channel length were thus neglected.

Plant growth and nutrient uptake was added as a sub-model in the NFT unit. Tomato plant growth was modelled for two separate parts: vegetative and fruits.

Vegetative growth was modelled following an ideal logistic function and calibrated against data on final vegetative weight. Fruit growth was modelled assuming a second order polynomial function and calibrated against production data. Total water uptake was modelled assuming a third order polynomial function. Data for calibration was obtained from control groups grown under ideal conditions in experiments at the Abtshagen system (Suhl, 2015) (see the Appendix of this chapter). Each nutrient uptake rate was assumed linearly related to the plant growth and the fruit composition.

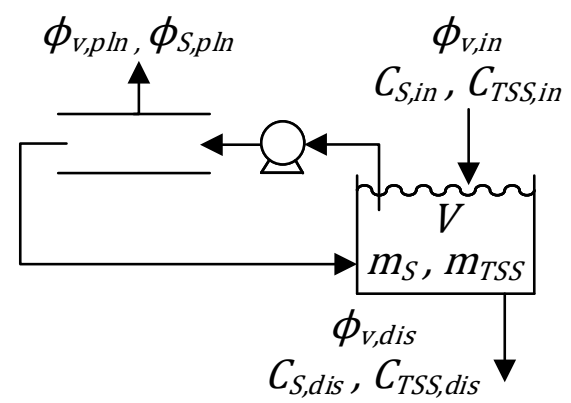

FIGURE 3.8: Model of the NFT tanks and channels. 


\subsubsection{Development of an improved management strategy}

The simulation results from the existing configuration of the aquaponic system in Abtshagen were analysed to identify opportunities for increased resource efficiency, using an improved management strategy, following the next steps:

1. Water balance. Identify the largest (waste) streams in the system, and possibilities for reuse on plants.

2. Nutrient balance. Identify surplus or deficit streams of nutrients in the RAS water, and possibilities for reuse on plants (compared to fertilizer use).

3. Water quality. Identify water quality parameters near or above suggested limits for fish and plant growth. These parameters become limiting factors for the water reuse potential identified in the previous steps.

4. Response surface analysis. Select system operation parameters affecting the streams identified in previous steps. List the critical water quality parameters (limiting factors). Run a set of simulations with a range of system operation parameters and evaluate their effect on resource efficiency, accounting for limiting factors from water quality. Identify an improved management strategy.

\subsection{Results and discussion}

The aquaponic system was simulated for a period of 2 years. Year 1 is the starting phase of the system. From year 2 onwards, the system operates with similar yearly behaviour. Therefore, yearly production and consumption were calculated based on year 2.

\subsubsection{Production}

Simulated growth and cumulative production of tilapia and tomato are shown in Fig. 3.9. After one start-up year, fish is harvested 8 times per year at $129.3 \mathrm{~kg}$ per harvest for a total of $1034 \mathrm{~kg} \mathrm{yr}^{-1}$ or $140 \mathrm{~kg} / \mathrm{m}^{3} \mathrm{yr}^{-1}$ with a final stocking density of $70 \mathrm{~kg} / \mathrm{m}^{3}$. This simulated yearly production is higher than commercial production $\left(60-120 \mathrm{~kg} / \mathrm{m}^{3} \mathrm{yr}^{-1}, \mathrm{FAO}, 2018 \mathrm{a}\right)$ due to the assumption of ideal growth with no mortality and expert management. At the time of this study, fish production in the actual system at Abtshagen was facing problems and comparison to simulated results is not possible. 
The model uses weekly harvest. The resulting tomato production was $4350 \mathrm{~kg}^{-1} \mathrm{r}^{-1}$ or $69 \mathrm{~kg} \mathrm{~m}^{-2} \mathrm{yr}^{-1}$ (net cultivated area), at $22.5 \mathrm{~kg} / \mathrm{plant}$. This simulated yearly production matches by calibration the conventional production under expert management (50 - $75 \mathrm{~kg} \mathrm{~m}^{-2} \mathrm{yr}^{-1}$, (De Gelder et al., 2005; Nederhoff and Stanghellini, 2010; Resh, 2016)). An experiment in the actual Abtshagen system without measures for increased productivity only achieved a production of $\left(32 \mathrm{~kg} \mathrm{~m}^{-2} \mathrm{yr}^{-1}\right.$ ) (Suhl et al., 2016), showing the relevance of expert management in our model assumptions.

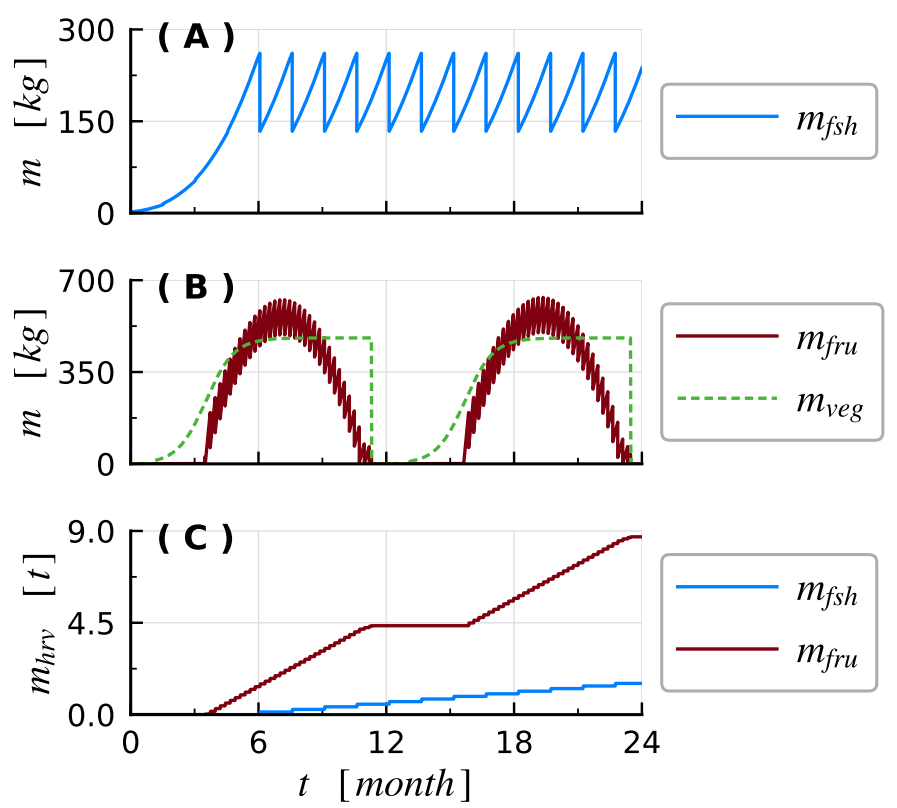

FIGURE 3.9: Simulated production. A) fish growth, B) plant growth.

C) cumulative harvest. After one start-up year, simulated production was $1034 \mathrm{~kg} \mathrm{yr}^{-1}$ for fish, and $4350 \mathrm{~kg} \mathrm{yr}^{-1}$ for tomato (fresh weights).

\subsubsection{Water balances}

Simulated cumulative water balances for the RAS and HS are shown in Fig. 3.10.

After one start-up year, the RAS consumes $994 \mathrm{~m}^{3} \mathrm{yr}^{-1}$ of fresh water, mainly to compensate for discharge from the PP $\left(900 \mathrm{~m}^{3} \mathrm{yr}^{-1}\right)$. Discharge from the PP happens when removing sludge or when it overflows. The main flow filling up the PP to eventual overflow comes from cleaning the ST. These large flows confirm the assumption for a negligible volume of water corresponding to fish body mass.

In the HS, fresh water consumption is negligible: $0.18 \mathrm{~m}^{3}$ during one start-up year, and $0 \mathrm{~m}^{3}$ afterwards. This low consumption in the HS happens because the system was simulated to prioritize water use from the RAS (fresh water is added to the MT only if its volume drops below $55 \%$ ). 


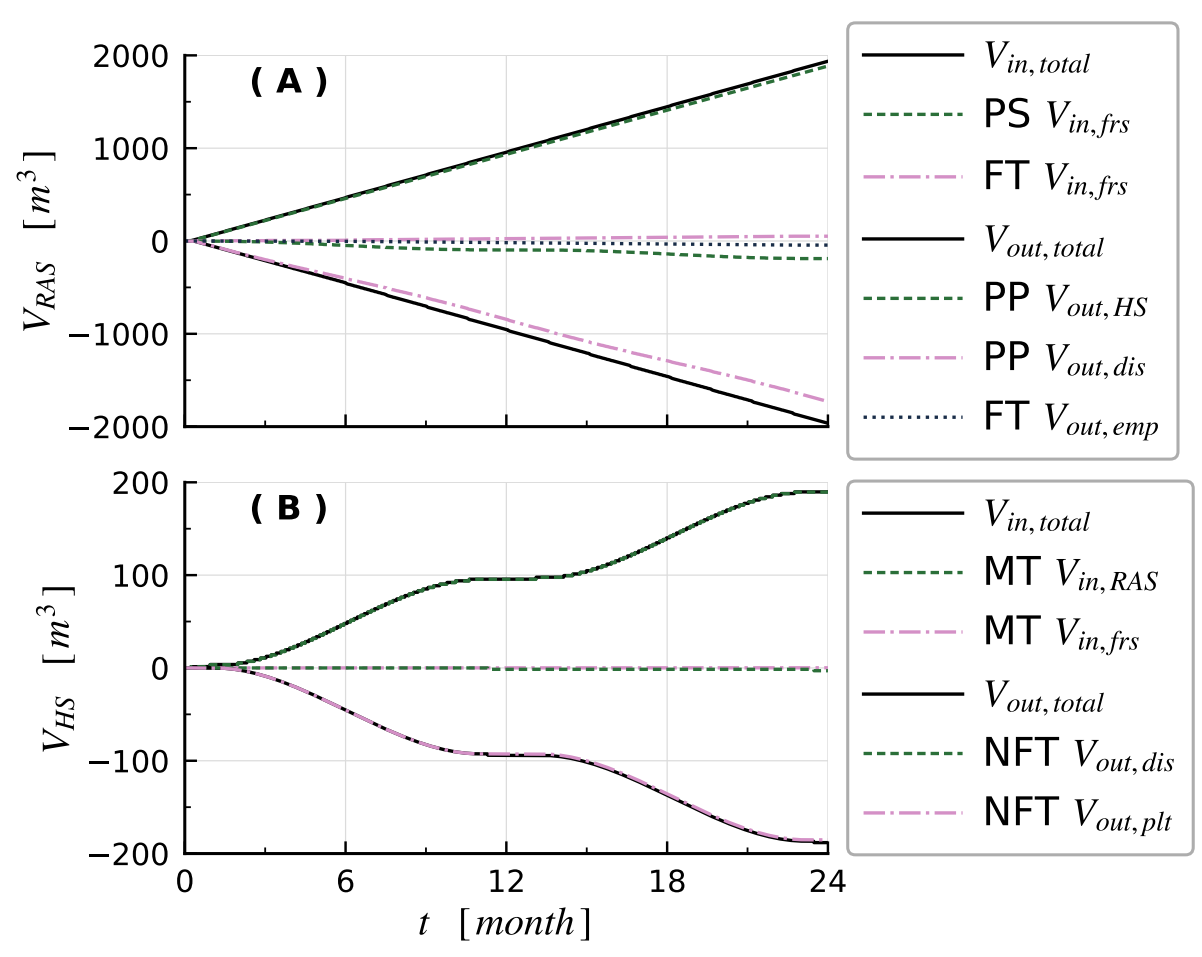

FIGURE 3.10: Simulated cumulative water balance. A) Recirculating aquaculture system (RAS). B) Hydroponic system (HS). Positive values represent water entering the system. Negative values represent water leaving the system. PS: pump sump. FT: fish tanks. PP: post-purge tank. MT: mixing tank for fertilizer. NFT: nutrient film technique channels and containers.

Assuming 95\% water content in tomato fruits (Suhl et al., 2016) and 70\% leaf relative water content (Tuna et al., 2007) (generalized for stem and roots), given a production of $4363 \mathrm{~kg} \mathrm{yr}^{-1}$ fruits and $480 \mathrm{~kg} \mathrm{yr}^{-1}$ vegetative, a total of $4481 \mathrm{~kg} \mathrm{yr}^{-1}$ water corresponds to plant weight $\left(4.48 \mathrm{~m}^{3} \mathrm{yr}^{-1}\right)$. This water content in the plant weight corresponds to $5 \%$ of the simulated yearly plant water uptake $\left(92.60 \mathrm{~m}^{3} \mathrm{yr}^{-1}\right)$, the rest being lost by evapotranspiration (88.12 $\left.\mathrm{m}^{3} \mathrm{yr}^{-1}\right)$. This evapotranspiration constitutes $9 \%$ of the total water consumption. Therefore, it is more important to reduce overall water input than to implement condensation recovery from the greenhouse.

The large amount of water discharged from the PP suggests that the RAS can provide water requirements for a much larger number of plants. But first, it is necessary to analyse the nutrient balances to determine the amount of nutrients that the fish water can provide to the plants. 


\subsubsection{Nutrient balances}

Simulated nutrient balances were focused on the HS, where nutrient addition can be reduced using RAS water. Fig. 3.11 shows the simulated nutrient balances in the HS.

The RAS supplies $25 \%$ of the plant $N$ requirements (TAN and $N O_{3}$ ). $P$ and $K$ are mostly supplied as fertilizer ( $P$ excretion by fish was assumed negligible). $\mathrm{Ca}, \mathrm{Mg}$ and $\mathrm{Na}$ are mostly supplied by RAS water. However, most of the $\mathrm{Mg}$ and $\mathrm{Na}$ enters the system in fresh water, not in fish feed. Excess $\mathrm{C} a, \mathrm{Mg}$ and $\mathrm{Na}$ is discharged in waste water at the end of the plant production cycle.

Based on excess nutrients and the water discharge from the PP, it could be suggested that fertilizer consumption for $\mathrm{N}, \mathrm{Ca}, \mathrm{Mg}$ and $\mathrm{Na}$ could be reduced using RAS water for an even larger number of plants. However, due to the excess of some nutrients, transferring a high amount of water from fish to plants could result in water quality conditions that may hinder plant growth. On the other hand, transferring low amounts of water could result in accumulation of nutrients that may hinder fish growth. Therefore, in the next section we analyse whether the water quality would be adequate for fish and plant growth.
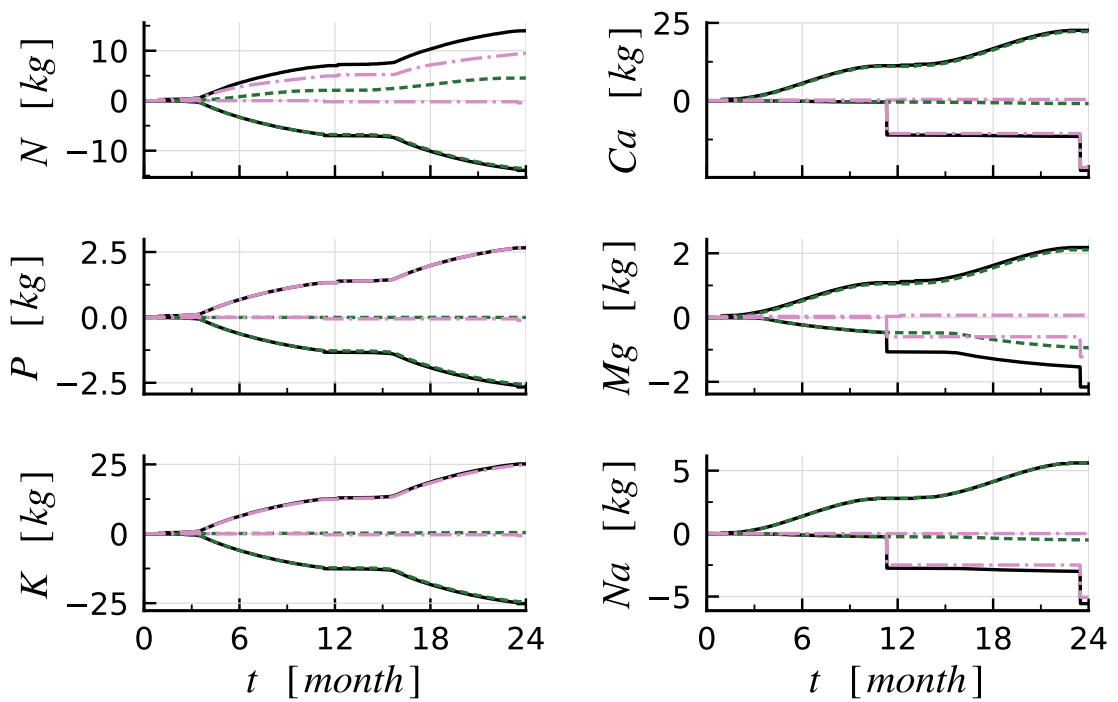

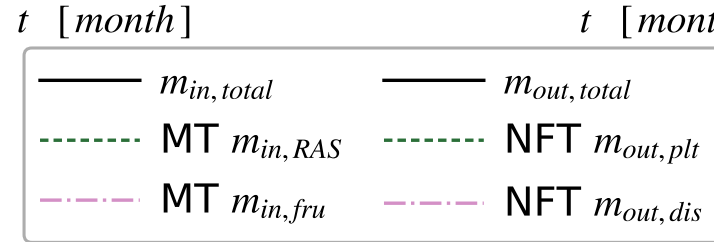

FIGURE 3.11: Simulated cumulative nutrient balances in the hydroponic system (HS).

Positive values represent nutrients entering the system in the mixing tank (MT).

Negative values represent nutrients leaving the system after harvest, discharged from the nutrient film technique (NFT) containers. $\mathrm{N}$ refers to TAN and $\mathrm{NO}_{3}-\mathrm{N}$. 


\subsubsection{Water quality}

According to the simulation, concentrations of some soluble nutrients and suspended matter can become limiting factors for the amount of water that can be sent from RAS to HS.

\section{RAS water quality}

Simulated nutrient concentrations in water of the first FT are shown in Fig. 3.12. These simulated concentrations contained high noise due to the recirculating nature of the system and because mixing dynamics was not modelled. Therefore, the resulting concentrations were filtered using an order 3 low pass Butterworth filter (normalized critical frequency 0.05) applied in a forward-backward linear digital filter (scipy functions signal.butter and signal.filtfilt). A comparison between filtered simulated values and recommended levels is shown in Table 3.2.
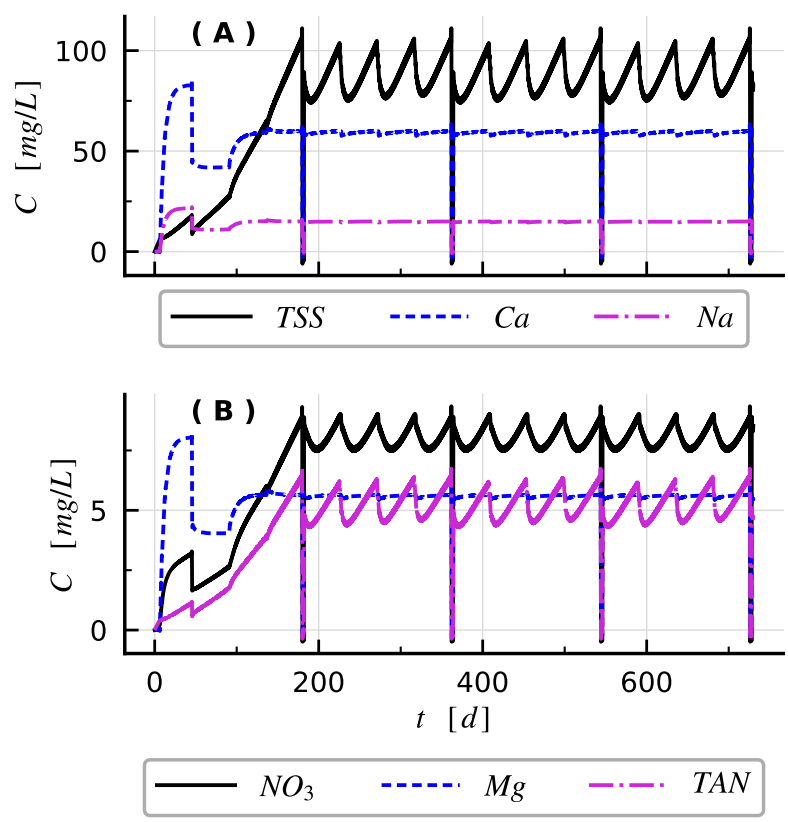

FIGURE 3.12: Simulated water quality in one fish tank (FT). A) TSS, Ca and Na. B) $\mathrm{NO}_{3}, \mathrm{Mg}$ and $\mathrm{TAN}$. Concentrations oscillate due to fish harvest every 45 days. Once every 180 days the FT depicted is harvested, shown as a concentration drop to 0 . $\mathrm{Ca}, \mathrm{Na}$, and $\mathrm{Mg}$ concentrations are mainly governed by fresh water addition, therefore they stabilize after starting up all 4 FTs. TSS, $\mathrm{NO}_{3}$, and TAN concentrations are mainly governed by fish excretion, therefore they oscillate with each harvest. 
TABLE 3.2: RAS water quality (after one start-up year). Simulated minimum \& maximum values vs. recommended levels

\begin{tabular}{lccl}
\hline Nutrient & $\begin{array}{c}\text { Simulated } \\
{[m g / L]}\end{array}$ & $\begin{array}{c}\text { Recommended } \\
{[m g / L]}\end{array}$ & References \\
\hline $\mathrm{TSS}$ & $73-104$ & $<200$ & {$[1,2,3]$} \\
$\mathrm{NO}_{3}$ & $7.5-9.0$ & $<300$ & {$[4]$} \\
$\mathrm{NH}_{3}$ & $0.023-0.036$ & $<0.1$ & {$[5]$} \\
$\mathrm{Ca}$ & $55-60$ & $50-160$ & {$[4,6]$} \\
$\mathrm{Mg}$ & 5.5 & $<15$ & {$[6]$} \\
$\mathrm{Na}$ & 15 & $<75$ & {$[6]$} \\
\hline
\end{tabular}

[1] Rakocy et al. (2004), [2] Dominguez et al. (2004)

[3] Ebeling and Vinci (2006), [4] DeLong et al. (2009)

[5] El-Shafai et al. (2004), [6] Timmons and Ebeling (2010)

During the start-up year, concentrations build up in the first FT and change suddenly every 45 days as tanks 2, 3, and 4 start operation. Afterwards, and every 180 days, the first FT is harvested. Once all 4 FT operate, one FT is harvested every 45 days. With every harvest, concentrations drop and subsequently build up with time due to fish waste, resulting in the characteristic saw-tooth pattern of a RAS. Concentrations of $\mathrm{Ca}, \mathrm{Na}$ and $\mathrm{Mg}$ show small oscillations because their levels are governed by fresh water (their input is higher from fresh water than from fish feed).

Attention must be given to TAN levels, because in the form of unionized ammonia $\left(\mathrm{NH}_{3}-\mathrm{N}\right)$ it is very toxic for fish. For tilapia, DeLong et al. (2009) suggested to maintain $\mathrm{NH}_{3}-\mathrm{N}$ below $1 \mathrm{mg} / \mathrm{L}$. However, El-Shafai et al. (2004), found negative effects of $\mathrm{NH}_{3}-\mathrm{N}$ on tilapia growth rate with long term exposure at concentrations as low as $0.144 \mathrm{mg} / \mathrm{L}$, and suggested levels below $0.1 \mathrm{mg} \mathrm{L^{-1 }}$. $\mathrm{NH}_{3}$ concentration depends on $\mathrm{pH}$ and TAN. Tilapia has been reported to grow best at $\mathrm{pH}$ between $6-9$. However, nitrifying bacteria requires a minimum $\mathrm{pH}$ of 6.8. Therefore, we assumed a controlled $\mathrm{pH}$ of 7.0 in the RAS. Under these conditions, based on $\mathrm{Ka}=\left[\mathrm{NH}_{3}-\mathrm{N}\right]\left[\mathrm{H}^{+}\right] /\left[\mathrm{NH}_{4}^{+}-\mathrm{N}\right]=10^{-9.25}$, and assuming activity of water equal to 1 (Metcalf-Eddy et al., 2014), the simulated TAN levels oscillating between $4.6-6.5 \mathrm{mg} \mathrm{L}^{-1}$ after the start-up year, are equivalent to $0.023-0.036 \mathrm{mg} \mathrm{L}^{-1}$ of $\mathrm{NH}_{3}-\mathrm{N}$. These levels are safe throughout the production cycle.

Attention must also be given to TSS levels, because the RAS only has one settling tank to remove solids, as opposed to the combination of mechanical filter and settling tank suggested in literature (Cripps and Bergheim, 2000). It has been suggested to maintain TSS levels below $80 \mathrm{mg} \mathrm{L}^{-1}$ in RAS (Timmons and Ebeling, 2010). Ebeling and Vinci (2006) reported growing fish in RAS with up to $100 \mathrm{mg} \mathrm{L}^{-1} \mathrm{TSS}$, in absence of other stress factors. However, tilapia has a high tolerance to solids, and it has been reported to grow at $200 m g L^{-1}$ (Dominguez et al., 2004) and up to $900 \mathrm{mg} \mathrm{L}^{-1}$ (Rakocy et al., 2004). We chose to aim for TSS levels below $200 \mathrm{mg} \mathrm{L}^{-1}$. 


\section{HS water quality}

Simulated nutrient concentrations in the NFT channels are shown in Fig. 3.13. A comparison between simulated and recommended levels is shown in Table 3.3.

Attention must be given to $\mathrm{NH}_{4}-\mathrm{N}$ levels. Similarly to the $\mathrm{NH}_{3}-\mathrm{N}$ in the RAS, its levels depend on $T A N$ and $\mathrm{pH}$, but in the case of tomato plants, we assumed controlled pH at 6 (Rakocy et al., 2006). Simulated TAN levels oscillate between $4-59 m g L^{-1}$ after 1 start-up year, equivalent to $56-825 m g L^{-1}$ of $N_{4}-N$. Borgognone et al. (2013) reported adverse effects on tomato production with decreased ratios of $\mathrm{NO}_{3}-\mathrm{N}: \mathrm{NH}_{4}-\mathrm{N}$ for $\mathrm{N}$ supply. They observed higher productivity with a 70:30 ratio. Based on this observation, and on the recommended levels for $\mathrm{NO}_{3}-\mathrm{N}$, we chose a maximum recommended concentration of $200 \mathrm{mg} \mathrm{L} \mathrm{L}^{-1}$ for $\mathrm{NH}_{4}-\mathrm{N}$ for this study. Simulated levels exceed this maximum recommended level by a factor of 4 .

Attention must also be given to levels of $\mathrm{Ca}, \mathrm{Mg}$, and $\mathrm{Na}$. $\mathrm{Ca}$ has been reported to reduce $K$ and $M g$ uptake, but tomato production was achieved at $C a$ levels up to 20 mM (800 mg L ${ }^{-1}$ ) (Paiva et al., 1998; Yamazaki et al., 1999). Mg has been reported to reduce fruit yield at $1 \mathrm{mM}(24 \mathrm{mg} / \mathrm{L})$, but under saline conditions negative effects appeared only at $5 \mathrm{mM}(121.5 \mathrm{mg} / \mathrm{L})$ (Carvajal et al., 1999). Na levels have been reported to cause stress on tomato plants at $50 \mathrm{mM}\left(1150 \mathrm{mg} \mathrm{L}^{-1}\right)$, but seedlings have been grown for 37 days with up to $100 \mathrm{mM} \mathrm{Na}$ with no significant differences from $50 \mathrm{mM} \mathrm{Na}$ nutrient solution (Shiyab et al., 2013). Simulated $\mathrm{Ca}$ levels exceed the maximum concentrations found in literature by up to a factor of 10 , and simulated $\mathrm{Na}$ is close to stress levels.

Although the water and nutrient balances suggest the possibility to increase the number of plants and the amount of water provided by the RAS, excess of $C a, M g$, and $\mathrm{Na}$ present limiting factors for the system. In the next section, different strategies to operate the water supply from RAS to HS are evaluated. 

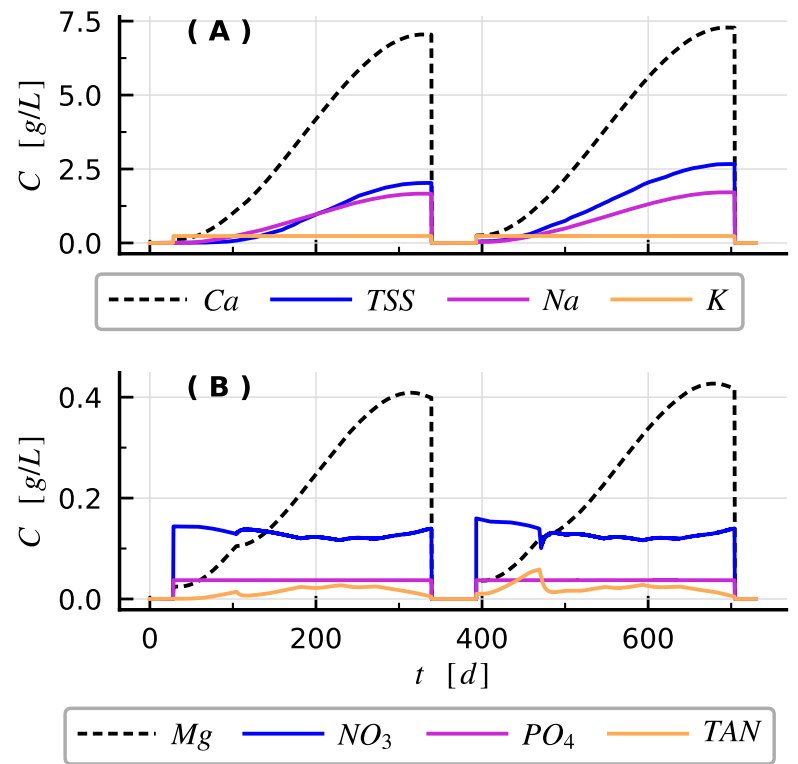

FIGURE 3.13: Simulated water quality in one NFT channel. A) $\mathrm{Ca}, \mathrm{TSS}, \mathrm{Na}$, and $\mathrm{K}$. B) $\mathrm{Mg}, \mathrm{NO}_{3}, \mathrm{PO}_{4}$, and TAN. The greenhouse stops production during winter, shown as a concentration drop to 0 . Plant requirements for $P$ and $K$ are not covered from RAS water, therefore their levels are almost entirely determined by the controlled addition of fertilizer (constant levels). Plant requirements for $N$ are partially covered by RAS, therefore its levels are partly determined by the addition of fertilizer and remain stable. Plant requirements for $\mathrm{Ca}, \mathrm{Mg}$ and $\mathrm{Na}$ are provided in excess by RAS water and they accumulate in the HS.

TABLE 3.3: NFT water quality.

Simulated maximum values vs. recommended levels.

\begin{tabular}{lccl}
\hline Nutrient & $\begin{array}{c}\text { Simulated } \\
{[m g / L]}\end{array}$ & $\begin{array}{c}\text { Recommended } \\
{[m g / L]}\end{array}$ & References \\
\hline $\mathrm{NO}_{3}-\mathrm{N}$ & 160 & $151-465$ & {$[1,2,3,4]$} \\
$\mathrm{NH}_{4}-\mathrm{N}$ & $56-825$ & $0.1-200$ & {$[1,2,3,4]$} \\
$\mathrm{P}$ & 37 & $15-66$ & {$[1,2,3,4]$} \\
$\mathrm{K}$ & 234 & $117-380$ & {$[1,2,3,4]$} \\
$\mathrm{Ca}$ & $255-7288$ & $100-800$ & {$[1,2,3,4,5,6]$} \\
$\mathrm{Mg}$ & 428 & $24-104$ & {$[1,2,3,7]$} \\
$\mathrm{Na}$ & $32-1713$ & $<1150$ & {$[8]$} \\
\hline
\end{tabular}

[1] Suhl et al. (2016), [2] Borgognone et al. (2013)

[3] Komosa et al. (2011), [4] Roosta and Hamidpour (2013)

[5] Zahedifar et al. (2012), [6] Paiva et al. (1998)

[7] Carvajal et al. (1999), [8] Shiyab et al. (2013) 


\subsubsection{Response surface analysis. Selecting a management strategy}

Water and nutrient balances from the previous sections suggested the possibility to reduce water and fertilizer addition by increasing 1) the number of plants and 2) the amount of water sent from RAS to HS. However, some concentrations in the FT and in the NFT channels may (further) exceed recommended levels when doing so. Therefore, we evaluated the effect of these system parameters on 1) Water consumption and discharge, and fertilizer consumption ( $N$ and $M g), 2)$ Concentration of TSS and $\mathrm{NH}_{3}-\mathrm{N}$ in FT as well as $\mathrm{NH}_{4}-\mathrm{N}, \mathrm{Ca}$, and $\mathrm{Na}$ in NFT channels. The objective was to select an improved system size and management strategy.

The model was simulated for different numbers of plants and for different ST cleaning intervals (related to the amount of water sent from RAS to HS). The number of plants was simulated for $92-768$ plants, and the shortest ST cleaning interval $\left(\Delta t_{\text {clean,min }}\right)$ was simulated for $0.5-6.5$ days. Both parameter ranges were split in 13 points, for a total of 169 simulations. The results are shown as contour plots of Fig. 3.14.

Fertilizer consumption was only analysed for $N$ and $M g$ because it can be reduced using RAS water (Fig. 3.14-left). Water quality was only analysed for the components that resulted in (near) excessive levels when simulating the given system configuration (Fig. 3.14-right).

Based on the contour plots in Fig. 3.14-left, it is first possible to determine that maximum $\mathrm{Ca}$ and $\mathrm{Mg}$ concentrations cannot be brought down to recommended levels. But it is still possible to decrease them using a lower number of plants (not desirable for improved productivity) or a higher ST cleaning interval. We therefore suggest to take additional measures to decrease $C a$ and $M g$ levels, such as filtration or an alternative fresh water source. However, minerals in the given fresh water source may be providing alkalinity that acts as a $\mathrm{pH}$ buffer, which may be the reason why $\mathrm{pH}$ control is not needed after the BF (nitrification would normally result in a $\mathrm{pH}$ drop).

Continuing with the analysis of the contour plots in Fig. 3.14-right, the main limiting factors are the concentrations of TSS in the FT, and $N a$ in the NFT channels. To maintain TSS concentrations within recommended levels for fish $(<200 \mathrm{mg} / \mathrm{L})$, the shortest interval for cleaning of the ST must be smaller than 2 days. And to maintain $\mathrm{Na}$ concentrations below excessive levels $(<2 \mathrm{mM}$ or $<2300 \mathrm{mg} / \mathrm{L}$ ), the number of plants can only be increased to about 222 plants. Tomato plants may be stressed at $1 \mathrm{mM}$ of $\mathrm{Na}$ but, based on Fig. 3.13, it would only be expected to reach the maximum simulated concentration during the last third of the plant growth period. 

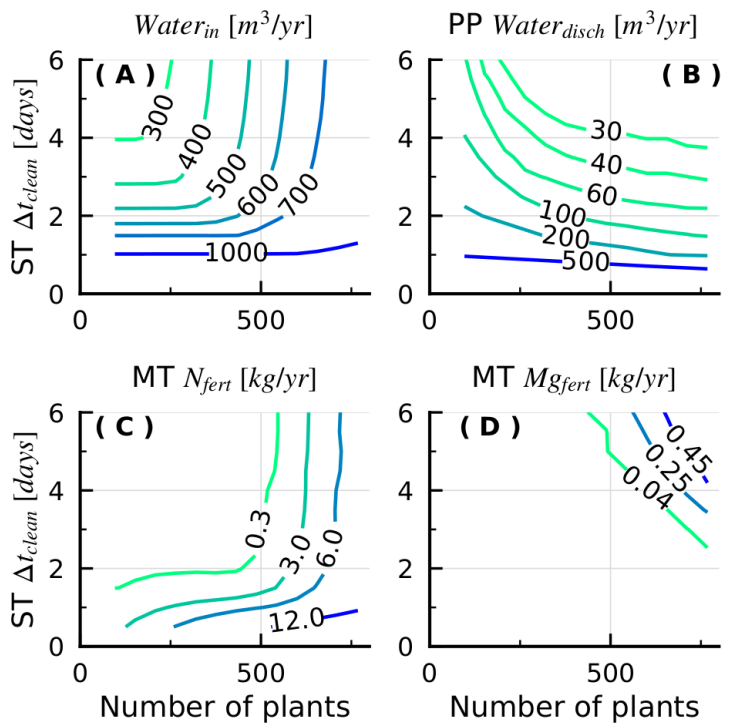
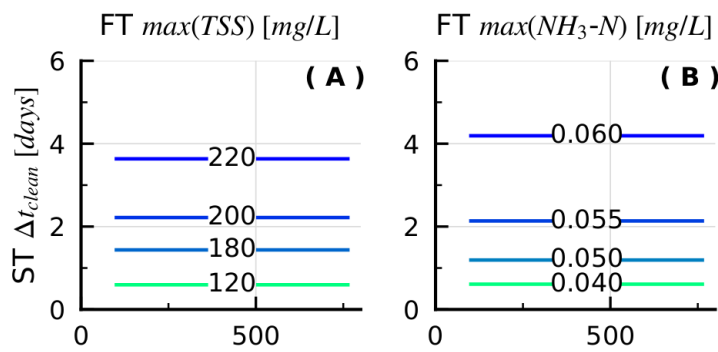

$\mathrm{NFT} \max \left(\mathrm{NH}_{4}-\mathrm{N}\right)[\mathrm{mg} / \mathrm{L}]$
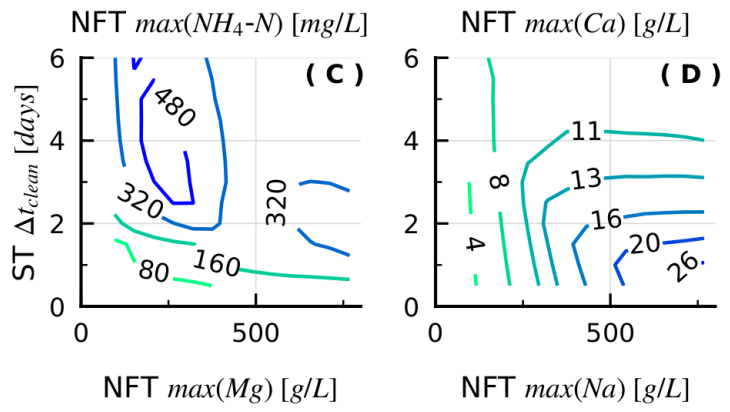

(D)
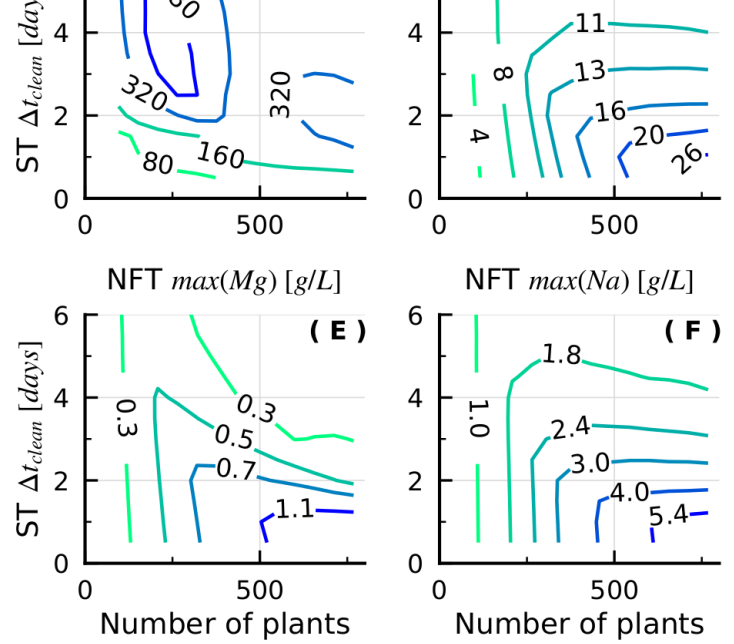

FIGURE 3.14: Response surface analysis from simulations.

Left. Effect of the number of plants and cleaning interval of the settling tank (ST) on yearly resource consumption. A) Fresh water addition to the system. B) Water discharge from the post-purge tank (PP). C) Fertilizer addition to the mixing tank (MT) for N. D) Fertilizer addition in the mixing tank (MT) for $M g$. The given system configuration operates with 192 plants, and a 0.5 day ST cleaning interval.

Right. Effect of the number of plants and cleaning interval of the settling tank (ST) on maximum concentrations. A) TSS in the fish tanks (FT). B) $\mathrm{NH}_{3}-\mathrm{N}$ in the fish tanks (FT). C) $\mathrm{NH}_{4}-\mathrm{N}$, in the NFT channels. D) $\mathrm{Ca}$ in the NFT channels. E) $\mathrm{Mg}$ in the NFT channels. F) $\mathrm{Na}$ in the NFT channels. The given system configuration operates with 192 plants, and a 0.5 day ST cleaning interval.

After fixing TSS and $\mathrm{Na}$ limits, the next limiting factors are $\mathrm{NH}_{4}-\mathrm{N}$ levels in the NFT channels (Fig. 3.14-right), together with water consumption and discharge (Fig. 3.14-left). Lowering the shortest ST cleaning interval decreases $\mathrm{NH}_{4}-\mathrm{N}$. However, it increases both water consumption and discharge. Increasing the maximum cleaning interval from 1.5 to 2 days could decrease water consumption by $150 \mathrm{~m}^{3} / \mathrm{yr}$, but it will not guarantee $\mathrm{NH}_{4}-\mathrm{N}$ levels for the NFT channels within recommended values. However, they would still decrease with respect to the given system configuration, from 825 to ca. $320 \mathrm{mg} / \mathrm{L}$. Furthermore, based on the NFT water quality (Fig. 3.13), TAN levels (and thus $\mathrm{NH}_{4}-\mathrm{N}$ ) only peak during the first third of the plant growth period and decrease afterwards. Therefore, it could be safe to operate at a 2 day ST 
cleaning interval.

Finally, fixing the HS size to 222 plants and the shortest ST cleaning interval to 2 days, fertilizer $N a$ and $M g$ addition is reduced to the minimum (Fig. 3.14-left), and $\mathrm{NH}_{3}-\mathrm{N}$ levels in the FT are still kept within recommended levels $(<0.1 \mathrm{mg} / \mathrm{L}$ ) (Fig. 3.14-right).

It is important to notice that even if $\mathrm{Ca}$ and $\mathrm{Na}$ are decreased from the fresh water input, which could allow for more plants, other variables would still get affected by an increase in the number of plants. $\mathrm{NH}_{4}-\mathrm{N}$ levels in the NFT channels could increase above the maximum recommended level (320 vs. $200 \mathrm{mg} / \mathrm{L}$ ). Beyond 500 plants, consumption of fertilizer $N$ would increase drastically (Fig. 3.14-right).

\subsubsection{Operation under improved management strategy}

The given aquaponic system size and management consisted of 192 plants and a shortest ST cleaning interval of 0.5 day. We found a new management strategy consisting of an increase to 222 plants (10 NFT channels with 22 plants each), and an increase of the shortest ST cleaning interval to 2 day. This new management strategy could improve the resource productivity and the water quality for fish and plants.

With the new management strategy, simulated tomato production increased from

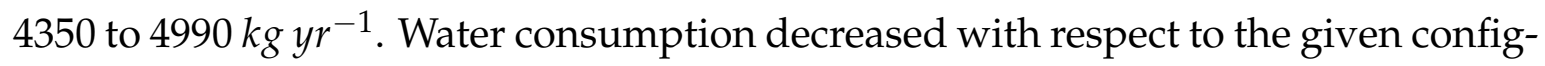
uration, from 994 to $380 \mathrm{~m}^{3} \mathrm{yr}^{-1}$ (272 $\mathrm{m}^{3} \mathrm{yr}^{-1}$ in the RAS and $108 \mathrm{~m}^{3} \mathrm{yr}^{-1}$ in the HS). Waste water discharge from the PP decreased from 900 to $164 \mathrm{~m}^{3} \mathrm{yr}^{-1}$. Fresh water consumption in the HS becomes necessary due to the reduced water discharge from the aquaponic system.

The simulated system productivity is summarized in Table 3.4. Water supply for fish can be similar to current commercial RAS systems in the Netherlands ( $240 \mathrm{~L} \mathrm{~kg}-1$ fish commercially). New developments in RAS such as denitrification could reduce water supply even further, to $40 \mathrm{~L} \mathrm{~kg}^{-1}$ fish (Martins et al., 2010), but this could reduce $N$ availability from fish to plants. Water supply for tomato can also be similar to some commercial systems in the Netherlands (21 $\mathrm{L} \mathrm{kg}^{-1}$ tomato). However, current production in closed greenhouse system can operate using as little as $4 \mathrm{~L} \mathrm{~kg}^{-1}$ tomato (Nederhoff and Stanghellini, 2010).

Nutrient supply to commercial greenhouse horticulture was calculated based on the range of water supply (Nederhoff and Stanghellini, 2010) and recommended levels for hydroponic solution. Simulated $P$ and $K$ requirements lie somewhere between the standard and high efficient closed greenhouse in the Netherlands. $\mathrm{N} \mathrm{Ca}$ and $M g$ requirements are one order of magnitude lower than those of commercial systems. 
Recirculating water for the NFT could help decreasing further $N, P$, and $K$ requirements. However, due to the high concentrations of other nutrients, this may result in stressful conditions for plant growth.

In summary, experimental-scale aquaponic production by the system in Abtshagen can operate with a resource efficiency that competes with large scale commercial production of tilapia and tomato in separate, specialized systems.

TABLE 3.4: Simulated resource productivity of the INAPRO aquaponic system in Abtshagen in its given and improved management strategies, compared to commercial RAS and greenhouse hydroponic production. INAPRO figures are

based on simulated results after 1 start-up year.

\begin{tabular}{llcccl}
\hline Resources & Units & $\begin{array}{c}\text { INAPRO } \\
\text { given }\end{array}$ & $\begin{array}{c}\text { INAPRO } \\
\text { improved }\end{array}$ & Commercial & References \\
\hline Fresh water & {$[\mathrm{L} / \mathrm{kg}$ fish $]$} & 961 & 263 & $40-240$ & {$[1]$} \\
Fresh water & {$[\mathrm{L} / \mathrm{kg}$ tomato $]$} & $\approx 0$ & 22 & $4-21$ & {$[2]$} \\
$N$ & {$[m g / \mathrm{kg}$ tomato $]$} & 1031 & 52 & $728-3822$ & {$[3,4]$} \\
$P$ & {$[m g / \mathrm{kg}$ tomato $]$} & 305 & 305 & $28-586$ & {$[3,4]$} \\
$K$ & {$[m g / \mathrm{kg}$ tomato $]$} & 2831 & 2831 & $782-4105$ & {$[3,4]$} \\
$\mathrm{Ca}$ & {$[m g / \mathrm{kg}$ tomato $]$} & 44 & 46 & $800-4200$ & {$[3,4]$} \\
$\mathrm{Mg}$ & {$[m g / \mathrm{kg}$ tomato $]$} & 8 & 9 & $292-1351$ & {$[3,4]$} \\
\hline
\end{tabular}

We do not compare water discharge and energy requirements to those in commercial systems because the INAPRO system in Abtshagen did not introduce changes for those aspects that could deviate from current production techniques.

[1] Martins et al. (2010). [2] Nederhoff and Stanghellini (2010)

[3] Ruijs (2011). [4] Sonneveld and Voogt (2009)

\subsubsection{Analysis of the system configuration}

The aquaponic system in Abtshagen has a small experimental size with unique characteristics.

- Solids removal in the RAS is only done in 1 settling tank, aiming at a reduced initial investment.

It has been suggested for a RAS to include a mechanical filtration unit, followed by a secondary sedimentation unit (Cripps and Bergheim, 2000).

As a result of the single settling tank (ST), the RAS in Abtshagen requires frequent cleaning and a large storage unit to store water for further use in the HS: the post-purge tank (PP). Large residence times in the PP may have led to anaerobic conditions which resulted in nitrogen losses by denitrifcation. Aeration of in the PP could help solving this problem (Suhl et al., 2016). 
Commercial-scale RAS operating with both mechanical filtration and sedimentation, would result in different water quality limitations when developing a management strategy.

- The RAS required no pH buffer during operation.

Controlling $\mathrm{pH}$ with buffers is frequently necessary after nitrification (Timmons and Ebeling, 2010). But in the Abtshagen system, using fresh water with high alkalinity may have helped controlling the $\mathrm{pH}$.

Commercial-scale systems may require the addition of a $\mathrm{pH}$ buffer, leading to higher concentrations of $\mathrm{Na}, \mathrm{Ca}$ or $\mathrm{K}$, depending on the buffer choice, which would also result in different water quality limitations when developing a management strategy.

\subsubsection{Limitations of the model}

This model assumed ideal growth for both fish and plants, provided by expert management. However, any deviation from ideal growth, could result in changes on the nutrient balances. These changes may require different parameters for the water and nutrient management strategy.

Plant nutrient uptake was modelled based on mineral composition of fruits, assuming that vegetative parts have the same composition and uptake. Therefore, predicted nutrient uptake may differ from reality during vegetative growth.

Settling of suspended solids was modelled linearly with respect to their mass in a tank. This could hold for low concentrations. But increased concentrations may result in lower settling than predicted.

Except for the BF, (bio)chemical reactions were neglected.

Measures for $\mathrm{pH}$ control were not modelled, such as addition of salts to increase alkalinity after nitrification in the BF.

The model was developed using a system-level approach and it was based on one existing aquaponic system. Further work is necessary to provide the model with more flexibility for the analysis of other systems. Particularly, detailed dynamics could improve the modularity of each component, allowing to change the overall system configuration while maintaining reliability in the simulations. To improve modularity, we suggest to model mixing dynamics in the tanks, particle size distribution effect on solids removal, effects of water quality on fish and plants, and possible reactions outside of the nitrification biofilter.

Finally, our selection for a management strategy was based on the response to 2 parameters. This allows to visualise the presence of local minima. To evaluate multiple parameters, we suggest the use of numerical algorithms for sensitivity analysis 
and optimisation (up to 5 parameters). This would first require the development of an objective function comprising water quality parameters and resource efficiency.

\subsection{Conclusions}

The main challenge in the development of aquaponics consists in balancing the requirements for growth of different species.

We developed a dynamic mathematical model with a system-level approach to understand water and nutrient dynamics, and improve resource efficiency. The model describes the dynamics of an existing aquaponic system with two connected loops based on the INAPRO configuration. Model simulations allowed to identify the operation parameters that have a significant impact on resource efficiency: number of plants and frequency of water exchange from fish to plants.

Sending more water from fish to plants can help reducing water and fertilizer consumption. However, it may also result in excessive concentrations of some nutrients, which may stress fish, plants or both. We developed a management strategy that achieved a balance between resource efficiency and water quality. With this strategy, we demonstrated the possibility to re-size and operate an existing aquaponic system in a way that improves its resource efficiency while maintaining the required water quality conditions.

Based on our simulations, water supply in the aquaponic system for fish and plant production (263 $\mathrm{L} \mathrm{kg}^{-1}$ fish and $22 \mathrm{~L} \mathrm{~kg}^{-1}$ tomato) is similar to commercial RAS and greenhouse hydroponic systems. $\mathrm{N}, \mathrm{Ca}$, and $\mathrm{Mg}$ supply for plant production (52, 46 and $9 \mathrm{mg} \mathrm{kg}^{-1}$ tomato, respectively) was one order of magnitude lower than in commercial systems. $P$ and $K$ supply was similar to current commercial systems. 


\subsection{Appendix}

\section{Plant growth calibration}

Calibration was done on the solution of the differential equation:

$$
\begin{aligned}
\frac{d m_{\text {veg }}}{d t} & =\mu m_{\text {veg }}\left(1-m_{\text {veg }} / m_{\text {veg, }, \text { ax }}\right) \\
m_{\text {veg }} & =\frac{n_{\text {plt }} m_{\text {veg, }, \text { max }} m_{\text {veg }, 0} e^{\mu\left(t-t_{0, v e g}\right)}}{m_{\text {veg, } \text { max }}+m_{\text {veg }, 0}\left(e^{\mu\left(t-t_{0, v e g}\right)}+1\right)}
\end{aligned}
$$

The function was fitted to the final mass $m_{v e g, \max }$ obtained in the Abtshagen site.

\section{Fruit growth calibration}

Fruit growth was modelled based on a least-squares, second order polynomial regression from data of average fruit harvest collected in the demonstration system in Abtshagen:

$$
m_{f r u}=n_{p l t} Y_{f r u}\left[p_{1, f r u}\left(t-t_{0, f r u}\right)^{2}+p_{2, f r u}\left(t-t_{0, f r u}\right)\right]
$$

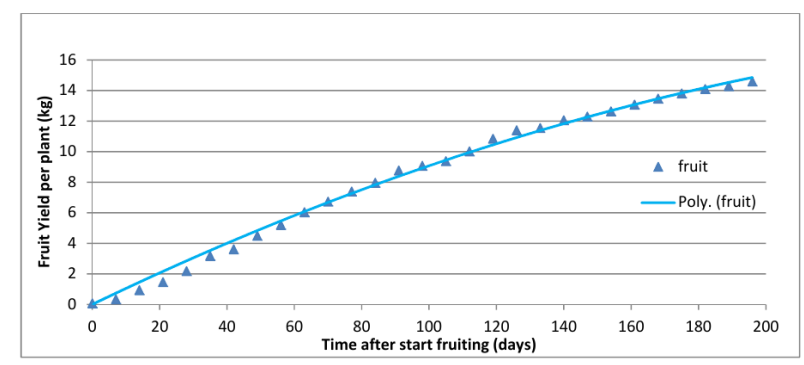

FIGURE A3.1: Least-squares regression for fruit production to a second order polynomial function. Data from Suhl (2015). 
Chapter 3. Model-based management strategy for resource efficient design and

\section{Plant water uptake calibration}

Water uptake by the plants was modelled based on a least-squares, third order polynomial regression from data of cumulative plant water uptake $\left(V_{p l}\right)$ in the demonstration system in Abtshagen, which allows to calculate the water uptake for each time-step $\left(\phi_{v, p l}\right)$

$$
\begin{aligned}
V_{w, p l t}=n_{p l t} Y_{f r u} m_{v e g, \max }[ & p_{1, w}\left(t-t_{0, v e g}\right)^{3} \\
& \left.+p_{2, w}\left(t-t_{0, v e g}\right)^{2}+p_{3, w}\left(t-t_{0, v e g}\right)\right] \\
\phi_{v, p l t}= & V_{w, p l}(t+1)-V_{w, p l t}(t)
\end{aligned}
$$

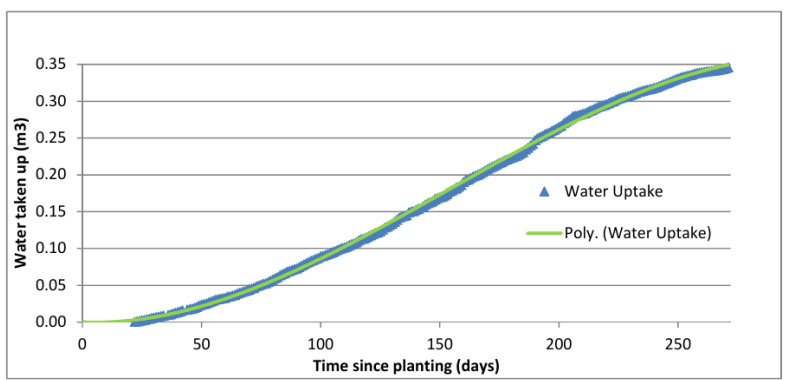

FIGURE A3.2: Least-squares regression for cumulative water uptake to a third order polynomial function. Data from Suhl (2015). 


\section{Mass balances}

TABLE A3.1: Mass balances for the models of each module (unit) in the aquaponic system (Eqn. 3.1). Variable names in each module are independent, even if shared between modules.

\begin{tabular}{|c|c|c|c|}
\hline Module & $d / d t$ & $\phi_{\text {in }}$ & $\phi_{\text {out }}$ \\
\hline FT & $\begin{array}{l}V \\
m_{S} \\
m_{T S S}\end{array}$ & $\begin{array}{l}\phi_{v, \text { in }}+\phi_{v, f r s} \\
C_{S, \text { in }} \phi_{v, \text { in }}+\phi_{S, f s h}+C_{S, f r s} \phi_{v, f r s} \\
C_{T S S, \text { in }} \phi_{v, \text { in }}+\phi_{T S S, f s h}\end{array}$ & $\begin{array}{l}\phi_{v, \text { out }}+\phi_{v, h r v}+\phi_{v, f s h} \\
\left(m_{S} / V\right) \phi_{v, \text { out }}+\left(m_{S} / V\right) \phi_{v, h r v} \\
\left(m_{T S S} / V\right) \phi_{v, \text { out }}+\left(m_{T S S} / V\right) \phi_{v, h r v}\end{array}$ \\
\hline ST & $\begin{array}{l}V \\
m_{S} \\
m_{T S S} \\
m_{T S S, \text { set }}\end{array}$ & $\begin{array}{l}\phi_{v, \text { in }} \\
C_{S, \text { in }} \phi_{v, \text { in }} \\
C_{T S S, \text { in }} \phi_{v, \text { in }}+\phi_{r s p} \\
\phi_{r e t}+\phi_{r s p}\end{array}$ & $\begin{array}{l}\phi_{v, \text { out }}+\phi_{v, \text { slg }} \\
\left(m_{S} / V\right) \phi_{v, \text { out }}+\left(m_{S} / V\right) \phi_{v, \text { slg }} \\
\left(m_{T S S} / V\right) \phi_{v, \text { out }}+\phi_{\text {ret }}+\phi_{v, \text { slg }} \\
m_{T S S, \text { set }} \phi_{v, \text { slg }}\end{array}$ \\
\hline PS & $\begin{array}{l}V \\
m_{S} \\
m_{T S S}\end{array}$ & $\begin{array}{l}\phi_{v, \text { in }}+\phi_{v, f i l t}+\phi_{v, f r s} \\
C_{S, \text { in }} \phi_{v, \text { in }}+C_{S, f l t} \phi_{v, f l t} \\
+C_{S, f r s} \phi_{v, f r s} \\
C_{T S S, \text { in }} \phi_{v, \text { in }}+C_{T S S, f l t} \phi_{v, f l t}\end{array}$ & $\begin{array}{l}\phi_{v, \text { out }} \\
\left(m_{S} / V\right) \phi_{v, \text { out }} \\
\left(m_{T S S} / V\right) \phi_{v, \text { out }}\end{array}$ \\
\hline $\mathrm{BF}$ & $\begin{array}{l}V \\
m_{S} \\
m_{T S S}\end{array}$ & $\begin{array}{l}\phi_{v, \text { in }}+r_{\mathrm{H}_{2} \mathrm{O}} A_{\mathrm{BF}} / \rho_{\mathrm{H} 2 \mathrm{O}}{ }^{\dagger} \\
C_{S, \text { in }} \phi_{v, \text { in }}+r_{T A N} A_{B F}{ }^{+} \\
C_{T S S, \text { in }} \phi_{v, \text { in }}\end{array}$ & $\begin{array}{l}\phi_{v, \text { out }}+\phi_{v, f l t} \\
\left(m_{S} / V\right) \phi_{v, \text { out }}+\left(m_{S} / V\right) \phi_{v, f l t} \\
\left(m_{T S S} / V\right) \phi_{v, \text { out }}+\left(m_{T S S} / V\right) \phi_{v, \text { filt }}\end{array}$ \\
\hline PP & $\begin{array}{l}V \\
m_{S} \\
m_{T S S}\end{array}$ & $\begin{array}{l}\phi_{v, s l g}+\phi_{v, h r v} \\
C_{S, s l g} \phi_{v, s l g}+C_{S, h r v} \phi_{v, h r v} \\
+C_{T S S, h r v} \phi_{v, h r v}\end{array}$ & $\begin{array}{l}\phi_{v, \text { out }}+\phi_{v, \text { dis }} \\
\left(m_{S} / V\right) \phi_{v, \text { out }} \\
\left(m_{S} / V\right) \phi_{v, \text { out }}+\phi_{\text {ret }}\end{array}$ \\
\hline MT & $\begin{array}{l}V \\
m_{S} \\
m_{T S S}\end{array}$ & $\begin{array}{l}\phi_{v, \text { in }}+\phi_{v, f r s} \\
C_{S, \text { in }} \phi_{v, \text { in }}+C_{S, f r s} \phi_{v, f r s}+\phi_{f r t} \\
C_{T S S, i n} \phi_{v, \text { in }}\end{array}$ & $\begin{array}{l}\phi_{v, \text { out }} \\
\left(m_{S} / V\right) \phi_{v, \text { out }} \\
\left(m_{T S S} / V\right) \phi_{v, \text { out }} \\
\end{array}$ \\
\hline NFT & $\begin{array}{l}V \\
m_{S} \\
m_{T S S}\end{array}$ & $\begin{array}{l}\phi_{v, \text { in }} \\
C_{S, \text { in }} \phi_{v, \text { in }} \\
C_{T S S, \text { in }} \phi_{v, \text { in }}\end{array}$ & $\begin{array}{l}\phi_{v, p l t}+\phi_{v, \text { dis }} \\
\phi_{S, p l t}+\left(m_{S} / V\right) \phi_{v, \text { dis }} \\
\left(m_{T S S} / V\right) \phi_{v, \text { out }}\end{array}$ \\
\hline
\end{tabular}

t We assumed that a chemical reaction only takes place in the BF (nitrification).

For simplicity, this term is added in the inflow column.

Stoichiometric coefficients in Table A3.3 reflect production and consumption. 


\section{Auxiliary equations}

TABLE A3.2: Auxiliary equations for the mass balances of each module in the aquaponic system. Variable names in each module are independent, even if shared between modules.

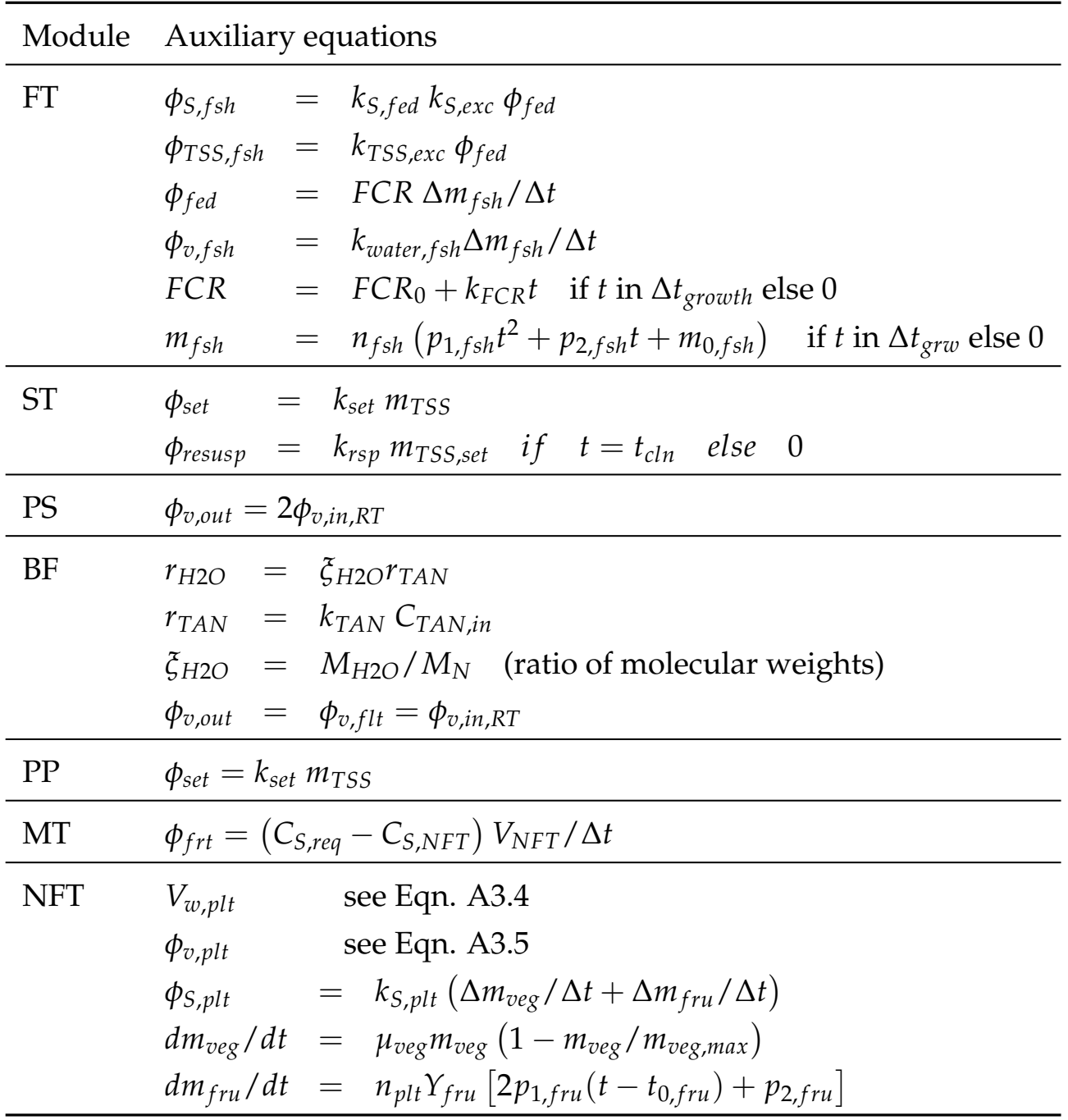




\section{Parameters}

TABLE A3.3: Parameters values used to model the aquaponic system.

\begin{tabular}{|c|c|c|c|c|}
\hline Module & Parameter & Value & Units & Description \\
\hline \multirow[t]{12}{*}{ FT } & $V$ & 1.84 & $m^{3}$ & $\begin{array}{l}\text { Max. volume of a fish } \\
\text { tank }\end{array}$ \\
\hline & $\phi_{v, \text { in }}$ & 3.68 & $m^{3} / h r$ & $\begin{array}{l}\text { Recirculating water } \\
\text { flowrate }\end{array}$ \\
\hline & $\Delta t_{g r w}$ & 180 & day & Fish growth period \\
\hline & $k_{w, f s h}$ & 0.8 & $\mathrm{~kg} / \mathrm{kg}$ & Water content in fish mass \\
\hline & $n_{f s h}$ & 175 & - & Number of fish per tank \\
\hline & $m_{0, f s h}$ & 0.01 & $k g$ & Initial mass of one fish \\
\hline & $p_{1, f s h}$ & $1.89 \times 10^{-3}$ & $\mathrm{~kg} / \mathrm{day}^{2}$ & $\begin{array}{l}\text { Parameters for fish } \\
\text { growth }\end{array}$ \\
\hline & $p_{2, f s h}$ & $6.41 \times 10^{-4}$ & $\mathrm{~kg} / \mathrm{day}$ & (estimated) \\
\hline & $F C R_{0}$ & 0.8 & $\mathrm{~kg} / \mathrm{kg}$ & $\begin{array}{l}\text { Initial feed conversion } \\
\text { ratio }\end{array}$ \\
\hline & $k_{S, f e d}$ & $\begin{array}{l}(0.064,0.010 \\
0.003,0.010 \\
0.001,0.002)\end{array}$ & $\mathrm{kg} / \mathrm{kg}$ & $\begin{array}{l}\text { Feed dry mass content } \\
(N, P, K, C a, M g, N a)\end{array}$ \\
\hline & $k_{S, e x c}$ & $\begin{array}{l}(0.32,0,0.02 \\
0.47,0.14,0.06)\end{array}$ & $\mathrm{kg} / \mathrm{kg}$ & $\begin{array}{l}\text { Mass excreted per } \\
\text { mass of nutrient eaten } \\
(N, P, K, C a, M g, N a)\end{array}$ \\
\hline & $k_{T S S, e x c}$ & 0.25 & $\mathrm{~kg} / \mathrm{kg}$ & $\begin{array}{l}\text { Mass excreted per mass of } \\
\text { feed eaten }\end{array}$ \\
\hline \multirow[t]{3}{*}{ ST } & $V$ & 1.32 & $m^{3}$ & Volume \\
\hline & $k_{\text {set }}$ & 0.97 & $1 /$ day & Solids settling constant \\
\hline & $k_{r s p}$ & 0.97 & $1 /$ day & $\begin{array}{l}\text { Solids resuspension con- } \\
\text { stant (during cleaning } \\
\text { only) }\end{array}$ \\
\hline PS & $V$ & 2.38 & $m^{3}$ & Volume \\
\hline \multirow[t]{2}{*}{ BF } & $k_{\text {TAN }}$ & $2.18 \times 10^{-3}$ & $m^{3} / m^{2} /$ day & $\begin{array}{l}\text { Reaction constant for } \\
\text { TAN (linear) }\end{array}$ \\
\hline & $A$ & 1020.4 & $m^{2}$ & $\begin{array}{l}\text { Surface area of the } \\
\text { biofilter }\left(V=2.29 m^{3},\right. \\
\left.a=445 m^{2} / m^{3}\right)\end{array}$ \\
\hline \multirow[t]{2}{*}{ PP } & $V$ & 9 & $m^{3}$ & Volume \\
\hline & $k_{\text {set }}$ & 1.8 & $1 /$ day & Solids settling constant \\
\hline
\end{tabular}


Chapter 3. Model-based management strategy for resource efficient design and operation of an aquaponic system

TABLE A3.4: Parameters values used to model the aquaponic system (continued).

\begin{tabular}{|c|c|c|c|c|}
\hline Module & Parameter & Value & Units & Description \\
\hline \multirow[t]{2}{*}{ MT } & $V$ & & $m^{3}$ & Volume \\
\hline & $C_{S, r e q}$ & $(151,37,234,128,2$ & $m g / L$ & $\begin{array}{l}\text { Target concentration for } \\
\text { plants }(\mathrm{N}, \mathrm{P}, \mathrm{K}, \mathrm{Ca}, \mathrm{Mg}, \\
\mathrm{Na})\end{array}$ \\
\hline \multirow[t]{14}{*}{ NFT } & $V$ & 0.3 & $m^{3}$ & Volume per NFT channel \\
\hline & $n_{p l t}$ & 192 & - & Total number of plants \\
\hline & $Y_{f r u}$ & 22.72 & $k g$ & Fruit yield per plant \\
\hline & $\mu_{v e g}$ & 0.05 & $1 /$ day & $\begin{array}{l}\text { Plant (logistic) growth } \\
\text { rate }\end{array}$ \\
\hline & $m_{v e g, \max }$ & 2.5 & $\mathrm{~kg}$ & $\begin{array}{l}\text { Maximum vegetative } \\
\text { mass per plant }\end{array}$ \\
\hline & $p_{1, w}$ & $-1.46 \times 10-9$ & $m^{3} / \mathrm{kg} / \mathrm{day}^{3}$ & Parameters for \\
\hline & $p_{2, w}$ & $6.75 \times 10-7$ & $m^{3} / \mathrm{kg} / \mathrm{day}^{2}$ & plant water uptake \\
\hline & $p_{3, w}$ & $-7.25 \times 10-6$ & $\mathrm{~m}^{3} / \mathrm{kg} / \mathrm{day}$ & (estimated) \\
\hline & $t_{0, v e g}$ & 28 & day & $\begin{array}{l}\text { Start time for plant } \\
\text { growth (days after } \\
01 / \text { Jan) }\end{array}$ \\
\hline & $t_{\text {end,veg }}$ & 339 & day & $\begin{array}{l}\text { End time for plant growth } \\
\text { (days after } 01 / \text { Jan) }\end{array}$ \\
\hline & $k_{S, p l t}$ & $\begin{array}{l}(140,26,252,9.8 \\
9.7,5.2) \times 10^{-5}\end{array}$ & - & $\begin{array}{l}\text { Mass fraction of fruits }(N, \\
P, K, C a, M g, N a)\end{array}$ \\
\hline & $p_{1, f r u}$ & $-9.51 \times 10^{-6}$ & $1 /$ day $^{2}$ & Parameters for \\
\hline & $p_{2, f r u}$ & $6.49 \times 10^{-3}$ & $1 /$ day & fruit growth (estimated) \\
\hline & $t_{0, f r u}$ & 104 & day & $\begin{array}{l}\text { Start time for first harvest, } \\
\text { days after } 01 / \text { Jan }\end{array}$ \\
\hline WT & $C_{S, f r s}$ & $\begin{array}{l}(3.1,0.04,0.054 \\
2.4,115.0,11.2 \\
30.1)\end{array}$ & $m g / L$ & $\begin{array}{l}\text { Concentrations in fresh } \\
\text { water }\left(\mathrm{NO}_{3}, \mathrm{TAN}, \mathrm{P}, \mathrm{K},\right. \\
\mathrm{C} a, \mathrm{Mg}, \mathrm{Na})\end{array}$ \\
\hline
\end{tabular}




\title{
Chapter 4
}

\section{Set-membership parameter estimation based on Voronoi vertices}

Published as:

D. Reyes Lastiri, H.J. Cappon, \& K.J. Keesman (2021). Set-membership parameter estimation based on Voronoi vertices. Environmental Modelling and Software, 143. DOI: https://doi.org/10.1016/j.envsoft.2021.105125

\begin{abstract}
Identification of complex systems often face structural modelling errors and limited or low quality data, which hinder statistical characterisations. An alternative is the set-membership approach, where errors are assumed unknown-but-bounded. Setmembership estimation aims to find a feasible parameter set (FPS), which produces model outputs that fit within given error bounds. Most algorithms are limited to linear models, small number of parameters, or to discrete approximation of the FPS. These limitations hinder parameter estimation for relatively complex systems.

We present an efficient sampling-based set-membership algorithm with low computational complexity that improves the coverage of a discrete approximation of the FPS, characterised by hyperspheres from a Voronoi diagram of the parameter space. Additionally, we suggest a measure for set-membership accuracy based on deviations between the given error bounds and the feasible model output set (FMOS). Our algorithm provides a balance between accuracy and computational complexity, and a tool to investigate practical identifiability.
\end{abstract}




\subsection{Introduction}

Mathematical models for biological and environmental systems often combine a priori knowledge and experimental data, requiring a calibration step to estimate the unknown parameters. Frequently, uncertainty of parameter estimates is based on a statistical characterisation of the error in experimental data.

However, biological and environmental systems often present the problems of structural modelling error, and limited availability or low quality of experimental data sets. Therefore, most often a statistical characterisation of the error is inappropriate (Norton, 1987; Keesman, 1990; Walter and Piet-Lahanier, 1990).

In this context of structural modelling error with limited and uncertain data, an alternative to the statistical characterisation is a so-called set-membership (SM) characterisation, where the errors are assumed to be unknown-but-bounded. In the SM approach, ranges of expected system outputs are predicted, instead of single values. These ranges account for the effect of uncertainty in data and model outputs (Norton, 1987; Keesman, 1990; Walter and Piet-Lahanier, 1990; Milanese et al., 1996; Diniz, 2013).

Given a $p$-dimensional parameter vector $\vartheta$, and the error between measurement $y$ and model output $f(\vartheta)$ defined as $e(\vartheta):=y-f(\vartheta)$, in a set-membership approach the error is assumed to be point-wise bounded by a fixed positive number, i.e. $\|e(\vartheta)\|_{\infty} \leq \varepsilon$. Within the SM approach, set-valued estimation algorithms aim to find an exact or approximate feasible parameter set (FPS). The FPS produces model outputs that fit inside the error bounds at all times: FPS $:=\left\{\vartheta \in \mathbb{R}^{p} \mid\|y-f(\vartheta)\|_{\infty} \leq \varepsilon\right\}$ (Milanese et al., 1996; Keesman, 2011; Cerone et al., 2014) (Fig. 4.1).

The simplest SM algorithms are based on models that are linear in the parameters. This type of algorithms is used in signal processing for e.g. adaptive filtering, providing reduced computational complexity by updating parameter estimates only when error bounds are exceeded (Diniz, 2013). Applying these methods to non-linear models, common in biological and environmental systems, would introduce additional linearisation steps.

In contrast, SM parameter estimation of non-linear dynamic systems is frequently applied to solve the problem of uncertain and limited data. Common algorithms rely on either interval-based or sampling-based methods.

For example, using an interval-based method, Marvel and Williams (2012) proposed a framework for SM estimation and experimental design to deal with small data sets in biological systems, based on set inversion via interval analysis (SIVIA) (Jaulin and Walter, 1993). Similarly, Rumschinski et al. (2010) proposed a partition algorithm for SM estimation and model invalidation to discriminate between competing models in biochemical reaction networks. Interval-based algorithms can 
A) Data and error bounds

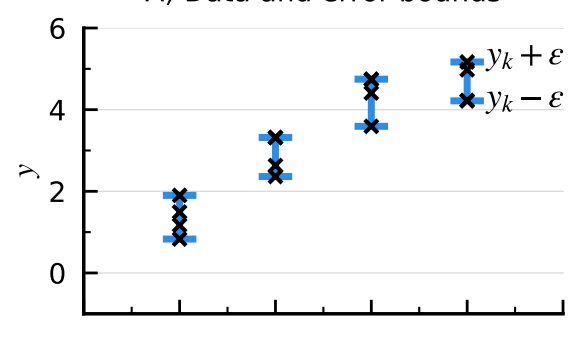

C) Feasible model output

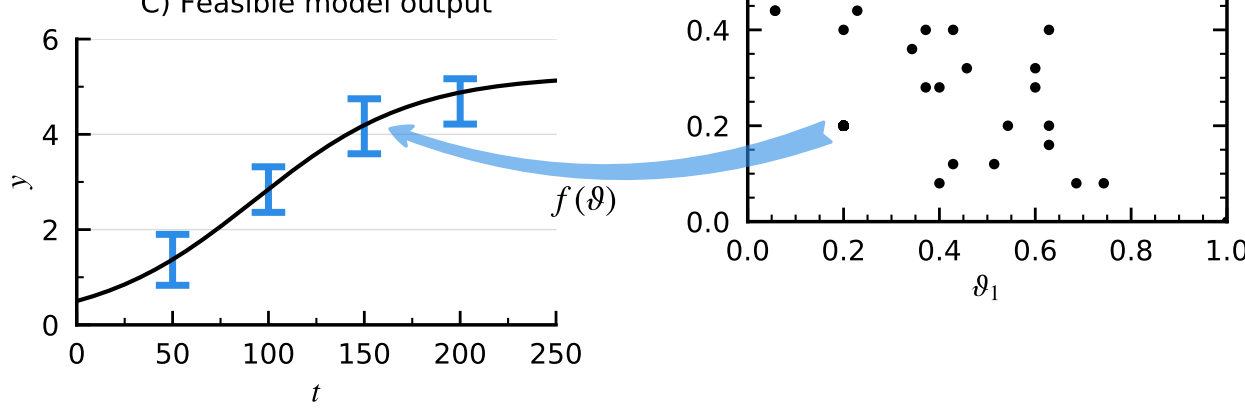

B) Parameter space with

feasible parameter vectors

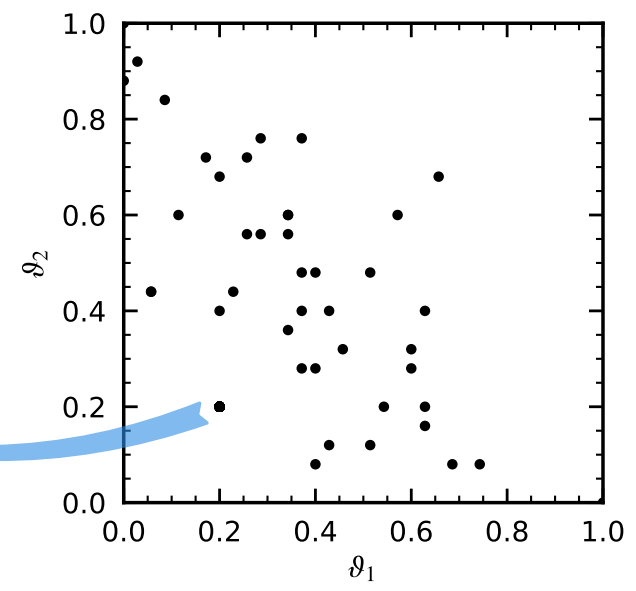

FIGURE 4.1: Set-membership (SM) parameter estimation.

A) Uncertainty in measured experimental data $(y)$ for each measurement instant

$(k)$ is defined by the error bounds of interval $\left[y_{k}-\varepsilon, y_{k}+\varepsilon\right]$. B) Some points in the space $\left(\mathbb{R}^{p}\right.$ with $\left.p=2\right)$ represent a parameter vector $(\vartheta)$ that produce model outputs that fit within the error bounds for all measurement instants $(k)$. C)

Example of a feasible model output $f(\vartheta)$ fitting within the bounds:

$\|y-f(\vartheta)\|_{\infty} \leq \varepsilon$. The objective in SM estimation is to characterise the set containing all feasible $\vartheta$ in $\mathbb{R}^{p}$, known as the feasible parameter set (FPS), with its corresponding feasible model output set (FMOS).

characterise the FPS for linear and non-linear models, and are capable of characterising non-convex parameter sets (Rumschinski et al., 2010), common in biological and environmental systems. The FPS characterised by interval-based methods is a collection of boxes with sizes determined by a desired accuracy. In general, they consist of a large number of boxes (hypercubes) that define the FPS boundary (ca. 20,000 boxes for a Lotka-Volterra test model with two estimated parameters by SIVIA (Marvel and Williams, 2012)). The number of boxes increases with the number of parameters and when higher accuracy is required (Jaulin and Walter, 1993). Furthermore, the number of model evaluations is proportional to the number of boxes characterising the FPS, resulting in an exponential computational complexity with respect to the number of parameters (Raïssi et al., 2009), i.e. a computational time with an order $O\left(n(\text { boxes })^{p}\right)$.

The FPS characterised by interval-based methods provides an accurate mathematical description that can be used to simulate a model and its prediction uncertainty. However, defining a large number of boxes and simulating from them is computationally demanding. 
In other examples, using sampling-based methods, Keesman (1990) proposed the characterization of the FPS by ellipsoids for more effective sampling. Van Straten and Keesman (1991) applied the so-called Monte Carlo set-membership algorithm (MCSM) to calibrate a model for lake eutrophication, and to simulate its prediction uncertainty from the resulting discrete FPS. Similarly, Nurulhuda et al. (2017) applied a Latin hypercube SM algorithm for a flooded rice system, returning a discrete approximation of the FPS in a six-dimensional space by means of relaxation of the error bounds. Given a fixed sample size per iteration, these algorithms have a linear computational complexity irrespective of the number of parameters, i.e. a computational time with an order $O(n$ (sample $))$.

The FPS characterised by sampling-based methods is less computationally demanding than interval-based methods. However, random sampling may require a large number of iterations. Therefore, low computational times and a full coverage of the FPS cannot be guaranteed.

Additionally, models of complex biological and environmental systems are often split into sub-models, see for example Van Straten and Keesman (1991), Mohtar et al. (1997), Mocenni and Vicino (2006), and Reyes Lastiri et al. (2018). Uncertainty in the parameter estimates often propagates through sub-models.

In uncertainty propagation studies, the statistical approach uses methods such as first-order variance propagation or Monte Carlo simulation. First-order variance propagation is hindered by the non-linearity of complex systems. Similarly, Monte Carlo simulation, which requires sampling from a probability density function ( $\mathrm{d} f$ ), is hindered by the uncertain and limited nature of experimental data, which may lead to inadequate $p d f$ characterisation.

Alternatively, in the SM approach, interval-based methods preserve the uncertainty characterization from the calibration step, but they are hindered by computational complexity. In contrast, sampling-based methods have less computational complexity, but simulating a discrete FPS without full coverage, and extending discrete feasible model output trajectories in time beyond the calibration interval, may lead to falsified predictions with underestimated uncertainty.

Consequently, given the aforementioned limitations, it is desired to have an efficient sampling-based algorithm that closely approximates the FPS, while preventing underestimation and overestimation of uncertainty propagation.

Therefore, the objective was to investigate and evaluate a novel set-membership estimation algorithm that meets the requirements of an uncertainty propagation study of complex systems with limited or low quality data. 


\subsection{Methods}

The proposed sampling-based SM estimation algorithm aims at efficiently characterising an approximate FPS, using a relatively low number of elements. Additionally, deviations from error bounds are quantified. The algorithm was evaluated in four test cases.

The algorithm was developed in Python 3.5, using the SciPy functions for Delaunay triangulation and Voronoi diagrams based on the Qhull library (Barber et al., 1996). The algorithm tests were run in the IPython console of WinPython 3.5 in an Intel Core i7-4790 CPU computer at 3.60 GHz with 16.0 GB RAM operating on Windows 7. The models were simulated with the 'odeint' function in the SciPy package (v. 0.18.1) of Python using its default parameters.

\subsubsection{Algorithm}

We propose characterizing the approximate FPS based on (hyper)spheres centred at Voronoi vertices. Parameters are assumed time-independent. The Voronoi diagram is generated using all known unfeasible parameter vectors as seeds, thus excluding them by definition.

Using spheres, complex FPS shapes can be approximated by two data vectors: centre coordinates $(c)$ and corresponding radii $(r)$. Therefore, non-convex parameter sets from non-linear models can be characterised with a relatively low number of elements.

Random sampling from spheres of the characterised FPS is computationally simple, and can then be used to simulate the model for $t \in[0, T]$, where $T$ is the final simulation time, including the calibration time interval. Such sampling-based method from a low number of elements allows for relatively low computational times.

Convergence of the algorithm is not defined in the parameter space $\mathbb{R}^{p}$, but in the model output space in terms of deviations between the approximate feasible model output set (FMOS) and the data error bounds for all measurement instants. In this way, although sampling-based, the algorithm tries to prevent under- and overestimation of prediction uncertainty.

\section{Voronoi diagram and Delaunay triangulation}

In a Voronoi diagram (Fig. 4.2), a space is partitioned into regions based on the distance to a specified set of points. For each of these points, called seeds, there is a corresponding Voronoi region (- -) where any point is closer to its seed than to any other seed. The Voronoi diagram for a set of points is dual to its Delaunay 
triangulation (-). In a Delaunay triangulation no point is inside the circumcircle of any triangle. The triangle circumcentres are also the Voronoi vertices. Our algorithm is based on a Voronoi diagram from the collection of all known unfeasible parameter vectors, thus excluding them from the approximate FPS.

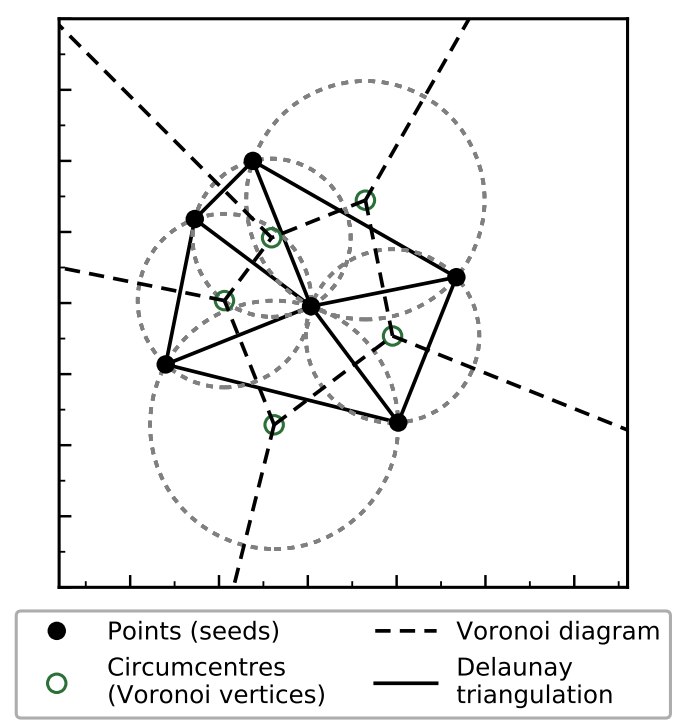

FIGURE 4.2: Voronoi diagram and Delaunay triangulation. The calculated Voronoi vertices are also circumcentres of circles that do not contain any of the given set of points (seeds).

\section{Definitions}

For the algorithm implementation, we define $\vartheta_{F}$ and $\vartheta_{U}$ as a feasible and unfeasible parameter vector, respectively. $\Omega_{\vartheta_{F}}$ and $\Omega_{\vartheta_{U}}$ are then the collections of all known feasible and unfeasible parameter vectors.

We also define $S_{\vartheta}$ as a random sample of parameter vectors from the set of spheres in $\mathbb{R}^{p}$. These sampled parameter vectors are evaluated individually to split the sample into a feasible $S_{\vartheta_{F}}$ and an unfeasible $S_{\vartheta_{U}}$ set of parameter vectors.

Furthermore, we define two types of deviations to measure accuracy of the approximate FPS at each measurement instant $k$. Overestimation $\left(O E_{k}\right)$ is the maximum deviation between model output $f(\vartheta)$ and error bounds, related to $\vartheta_{U}$ sampled in the FPS. Similarly, underestimation $\left(U E_{k}\right)$ is the maximum deviation between model output $f(\vartheta)$ and error bounds, related to all known $\vartheta_{F}$. Thus, for measurement instants $k=1, \ldots, M$, we define:

$$
\begin{aligned}
O E_{k} & :=\max _{i_{U}}\left[f\left(\vartheta_{U, i_{U}}\right)_{k}-\left(y_{k}+\varepsilon\right)\right]+\max _{i_{U}}\left[\left(y_{k}-\varepsilon\right)-f\left(\vartheta_{U, i_{U}}\right)_{k}\right] \\
U E_{k} & :=\min _{i_{F}}\left[\left(y_{k}+\varepsilon\right)-f\left(\vartheta_{F, i_{F}}\right)_{k}\right]+\min _{i_{F}}\left[f\left(\vartheta_{F, i_{F}}\right)_{k}-\left(y_{k}-\varepsilon\right)\right]
\end{aligned}
$$


Notice that $O E_{k}$ is calculated for a sample of unfeasible parameters with size $n\left(S_{\vartheta_{U}}\right)$ obtained from the approximate FPS in each iteration, i.e. $i_{U}=1, \ldots, n\left(S_{\vartheta_{U}}\right)$. In contrast, $U E_{k}$ is calculated for the set of size $n\left(\Omega_{\vartheta_{F}}\right)$ of all known feasible parameters up to the current iteration, i.e. $i_{F}=1, \ldots, n\left(\Omega_{\vartheta_{F}}\right)$. Fig. 4.3 illustrates these definitions.

Total $O E$ and $U E$ are defined as the sum of deviations for all measurement instants $(M)$ :

$$
\begin{aligned}
& O E:=\sum_{k=1}^{M} O E_{k} \\
& U E:=\sum_{k=1}^{M} U E_{k}
\end{aligned}
$$

Finally, we define the scalar-valued weighted deviation $(W D)$ from $O E$ and $U E$. $O E$ is weighted for the number of $\vartheta_{U}$ found in a sample inside the collection of spheres $\left(S_{\vartheta_{U}, i n}\right)$ at the corresponding iteration. UE is weighted for the number of $\vartheta_{F}$ found in a sample outside but near the collection of spheres $\left(S_{\vartheta_{F}, \text { out }}\right)$ at the corresponding iteration. Consequently:

$$
W D:=\left[n\left(S_{\vartheta_{u}, \text { in }}\right) / n\left(S_{\vartheta, \text { in }}\right)\right] O E+\left[n\left(S_{\theta_{F}, \text { out }}\right) / n\left(S_{\vartheta, \text { out }}\right] U E\right.
$$

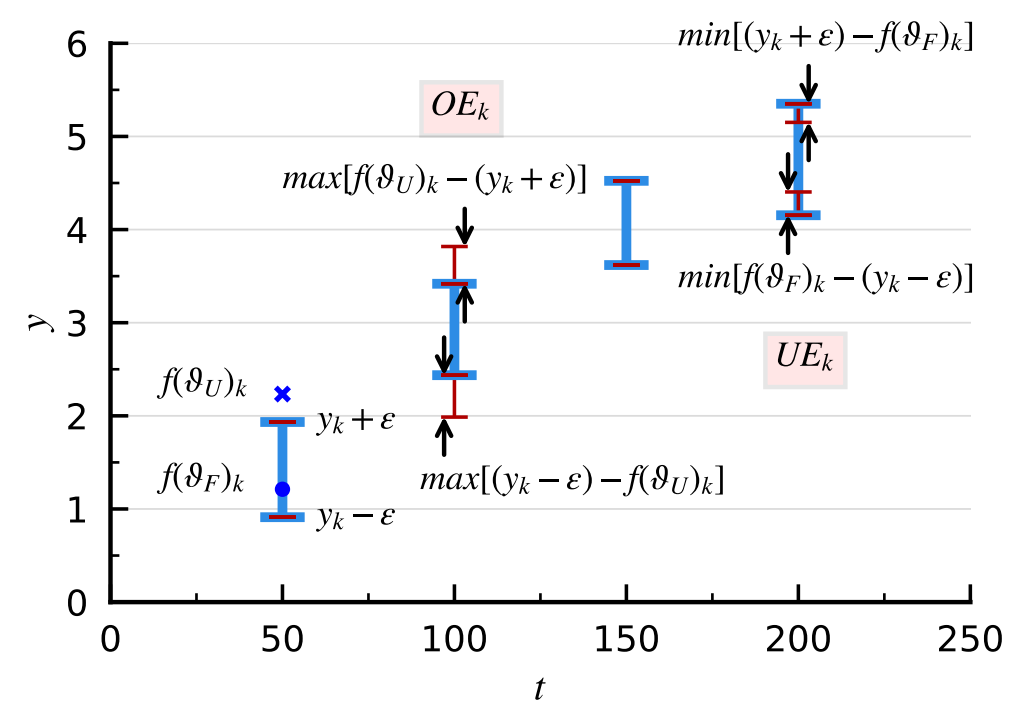

FIGURE 4.3: Overestimation (OE) and underestimation (UE).

At each measurement instant $k$, bounds are given by $y_{k}+\varepsilon$ and $y_{k}-\varepsilon$. An example of feasible $\left(f\left(\vartheta_{F}\right)\right)$ and unfeasible $\left(f\left(\vartheta_{U}\right)\right)$ model outputs is shown at $k=50 . O E_{k}$ shown for $k=100$ is the sum of deviations outside the data error bounds. $U E_{k}$ shown for $k=200$ is the sum of deviations inside the data error bounds. Convergence of the algorithm is based on reducing $O E_{k}$ and $U E_{k}$ for all $k$, to a given tolerance value for the total weighted deviation $\left(W D_{t o l}\right)$. 


\section{Implementation}

The algorithm consists of a main function 'Estimate' and four sub-functions: 'Initialize', 'Generate spheres', 'Sample', and 'Evaluate'. Implementation in pseudocode is shown in Algorithms 1 and 2. The functions are illustrated in Fig. 4.4 and described below. Supporting functions are provided in Algorithm A4.1 in the Appendix of this chapter. The approximate FPS is characterised by sets of centers and radii of spheres $(c, r)$.

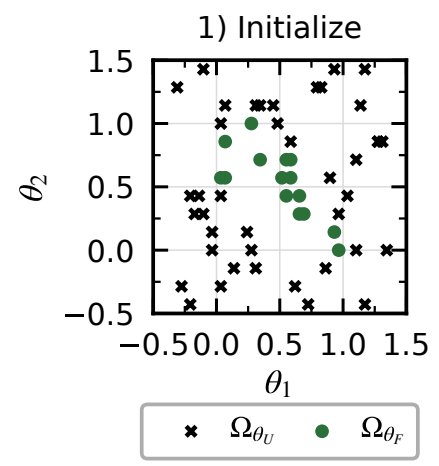

3) Sample

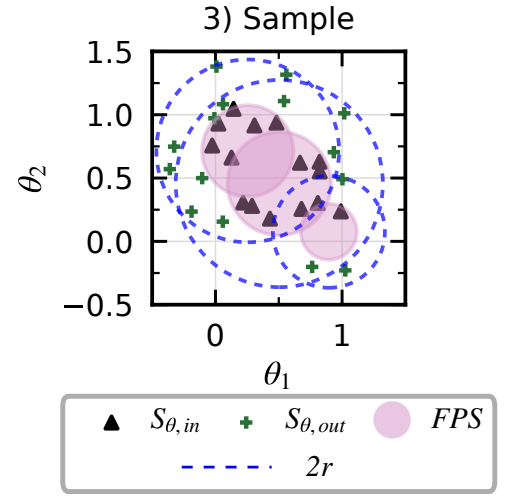

2) Generate spheres

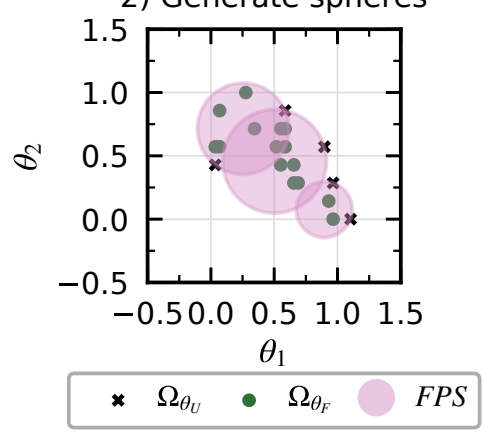

4) Evaluate

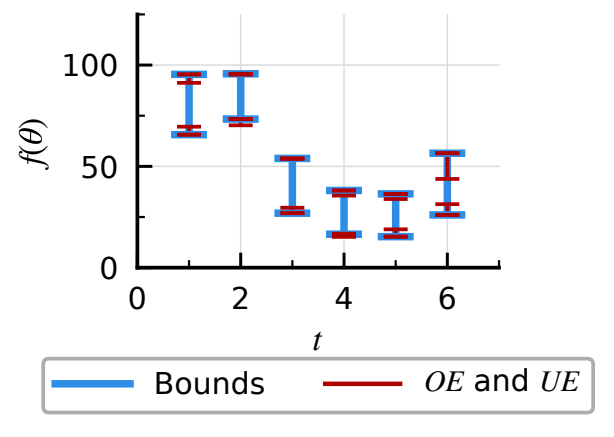

FIGURE 4.4: Algorithm functions. Example on a parameter space $\mathbb{R}^{p}$ with $p=2$.

1) Latin hypercube sampling is applied on $\mathbb{R}^{p}$. Normalization of $\mathbb{R}^{p}$ helps enclosing the approximate FPS near the interval $[0,1]$ for all coordinates.

2) A Voronoi diagram is generated with $\Omega_{\theta_{U}}$ as seeds. Subsequently, spheres are generated with centres in Voronoi vertices. In the first iteration, only $\theta_{U}$ circumscribed in the approximate FPS spheres are kept.

3) Two samples are obtained from the approximate FPS. The first sample inside the sphere set $\left(S_{\theta, i n}\right)$ searches for new $\theta_{U}$. The second sample outside the sphere set $\left(S_{\theta, \text { out }}\right)$ searches for new $\theta_{F}$ at a distance up to $2 r$ from each sphere.

4) Both samples are evaluated and split into feasible $\left(S_{\theta, F}\right)$ and unfeasible $\left(S_{\theta, U}\right)$, and appended to $\Omega_{\theta_{F}}$ and $\Omega_{\theta_{U}}$, respectively. Subsequently, $O E$ and $U E$ are calculated to determine WD (Eqn. 4.3). 


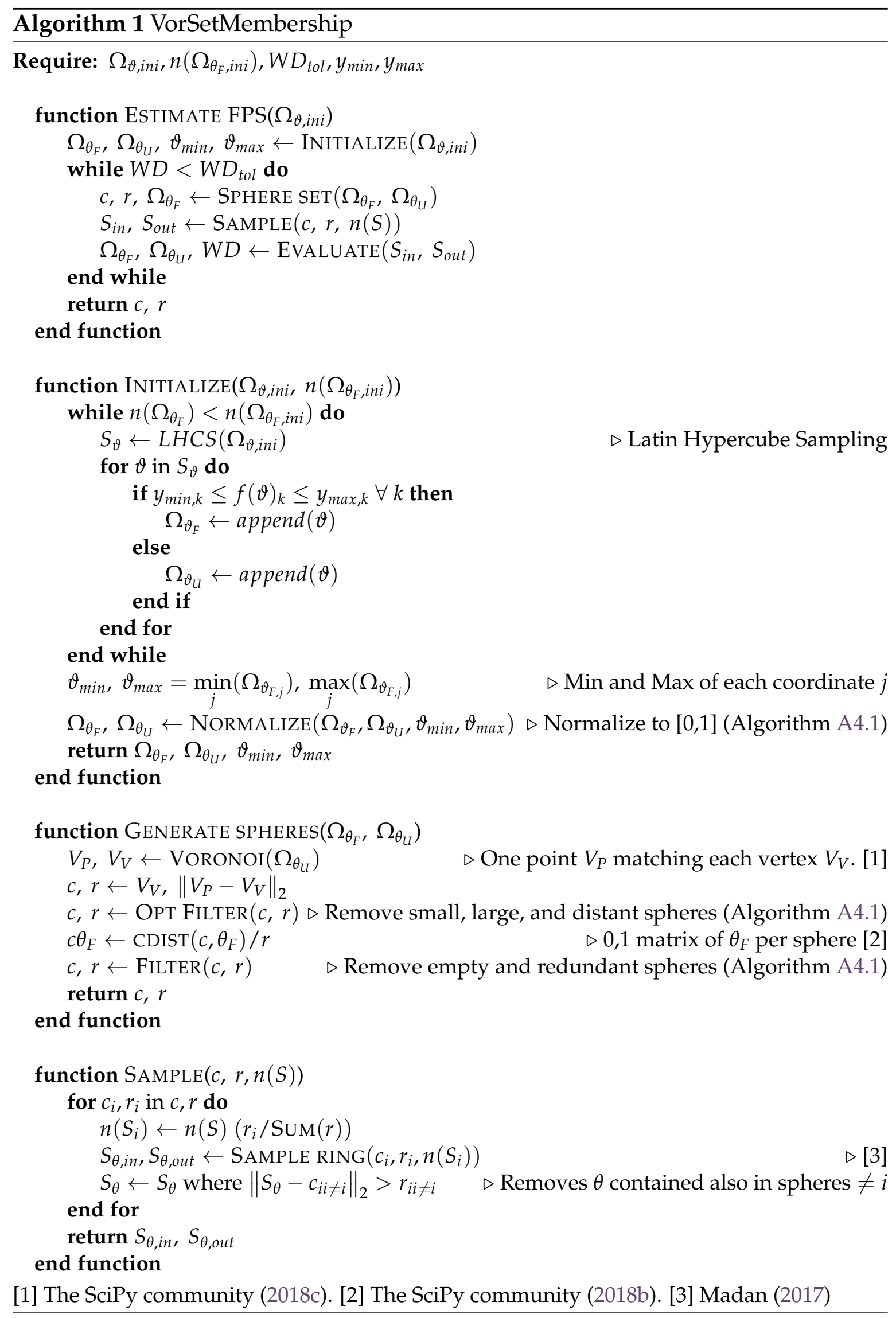




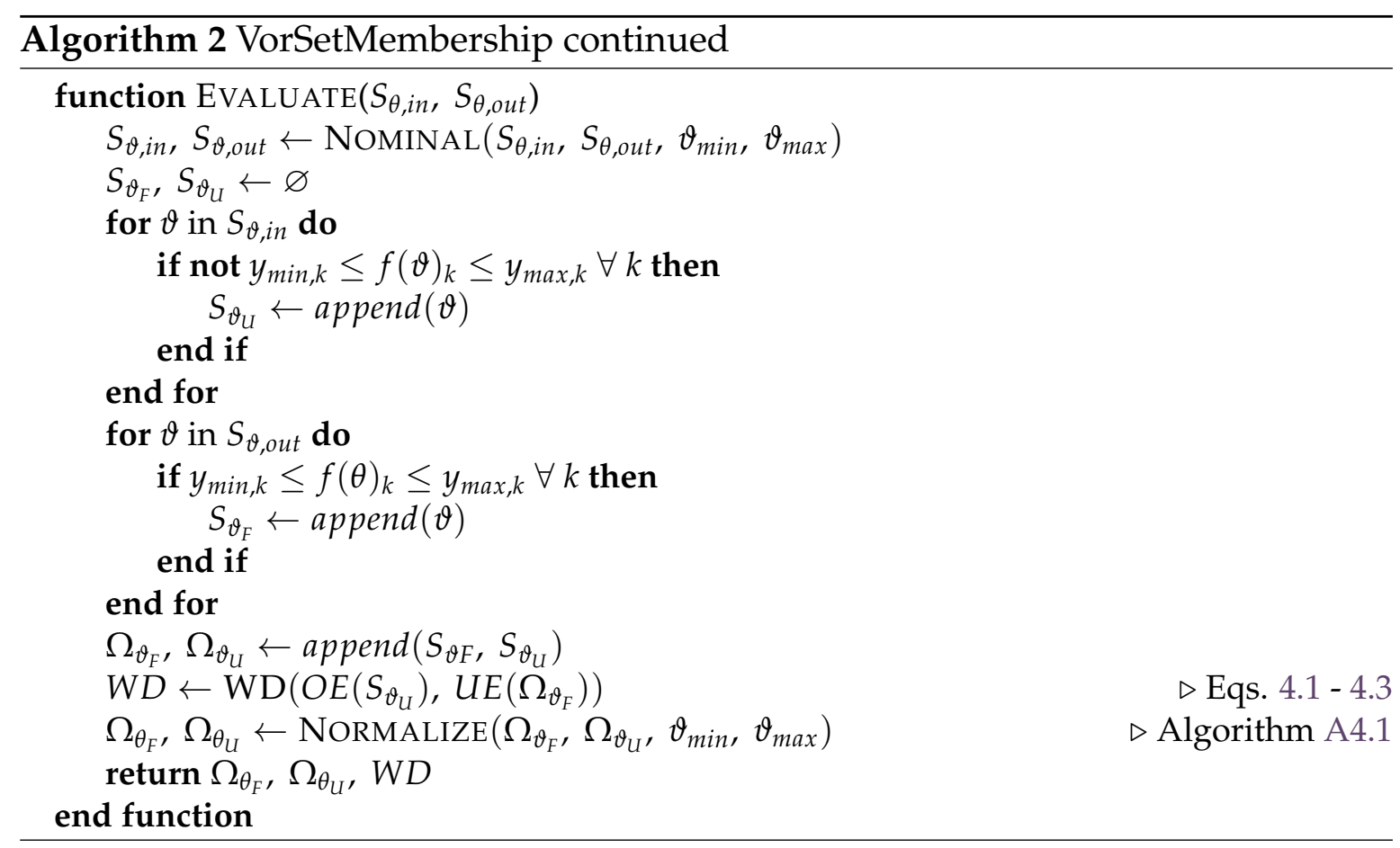

1) Initialize (Fig. 4.4.1): The sample set is initially defined as a (hyper)cube grid $\left(\Omega_{\vartheta, \text { ini }}\right)$. Latin hyper-cube sampling is applied iteratively on the grid $\Omega_{\vartheta, \text { ini }}$, evaluating $N_{\text {ini }}$ points per iteration. Sampled points are evaluated as feasible or unfeasible, initializing the sets $\Omega_{\vartheta_{F}}$ and $\Omega_{\vartheta, U}$. Sampling and evaluation continues until a desired size of $\Omega_{\vartheta_{F}}$ is obtained. The sets are subsequently normalized to $\Omega_{\theta_{F}}$ and $\Omega_{\theta_{U}}$ based on the minimum and maximum feasible coordinates found in the sample. Normalization helps maintaining the approximate FPS near the range $[0,1]$ for all parameters. If not enough $\vartheta_{F}$ or $\vartheta_{U}$ are found, the algorithm must be restarted. This step is purely random, and therefore governed by the choice of parameter ranges. To decrease randomness, it is best to have prior knowledge on the model behaviour, and parameters with physical meaning (constrained to a certain region). If no prior knowledge is available, a large $\Omega_{\vartheta, \text { ini }}$ with large $N_{i n i}$ can be used, which is subsequently narrowed by trial and error using this step alone.

2) Generate spheres (Fig. 4.4.2): A Voronoi diagram is generated using the SciPy function 'scipy.spatial.Voronoi' (The SciPy community, 2018c), with $\Omega_{\theta_{U}}$ as seeds. The Voronoi vertices generated constitute sphere centres. By definition, those spheres exclude any known $\theta_{U}$. Given minimum and maximum radii $\left(r_{\min }, r_{\max }\right)$, and constraints based on prior knowledge $\left(\vartheta_{\text {min }}, \vartheta_{\text {max }}\right)$, an optional filter can be applied to remove spheres with $r<r_{\text {min }}$ and $r>r_{\max }$, and spheres with any centre coordinate $c_{j}<\vartheta_{\text {min, } j}$ and $c_{j}>\vartheta_{\text {max }, j}$. Subsequently, spheres that do not contain any $\theta_{F}$ are removed. Finally, redundant spheres are also removed, i.e. spheres containing only $\theta_{F}$ included in larger spheres. These filters ensure that the number of spheres is kept low and their size is kept large, reducing memory use. In the first 
iteration, the set $\Omega_{\theta_{U}}$ is reset to contain only $\theta_{U}$ on which spheres with $\theta_{F}$ are circumscribed. This reduces the amount of data stored and focuses the next steps on the region where $\theta_{F}$ are found.

3) Sample (Fig. 4.4.3): Two random uniform samples of parameter vectors with size $n(S)$ are obtained from the set of spheres. Both samples are achieved by combining a normal distribution and its inverse (Madan, 2017). The first sample $\left(S_{\theta, i n}\right)$ is obtained inside the spheres to search for new $\theta_{U}$. The second sample $\left(S_{\theta, \text { out }}\right)$ is obtained in a ring outside but near the spheres, with radii ranging from $r$ to $2 r$, to search for new $\theta_{F}$. Redundant $\theta$ sampled from overlapping spheres are discarded, helping both samples to concentrate near the approximate FPS boundary to improve its accuracy.

4) Evaluate (Fig. 4.4.4): Feasibility of sampled parameter vectors is evaluated for both samples $S_{\theta, \text { in }}$ and $S_{\theta, \text { out }}$. Weighted deviation $(W D)$ is calculated from Eqn. 4.3. The algorithm stops if $W D \leq W D_{t o l}$. Otherwise, unfeasible parameter vectors found inside the approximate $F P S\left(S_{\theta_{i n, U}}\right)$ are appended to the set $\Omega_{\theta_{U}}$, feasible parameter vectors found outside the approximate $F P S\left(S_{\theta_{\text {out }, \mathrm{F}}}\right)$ are appended to the set $\Omega_{\theta_{F}}$, and a new iteration starts.

\subsubsection{Test model cases}

The algorithm was tested in four cases based on three non-linear dynamic models with biological and environmental components: a Lotka-Volterra population model, a microbial growth and degradation with under Monod kinetics, and the grass growth model in GRASIM (Mohtar et al., 1997).

For each case, artificial noisy measurements were generated around the base model outputs using a zero-mean uniform distribution with $\varepsilon=(1 / 4) \bar{y}$, where $\bar{y}$ is the time-averaged value of the model output. All model cases were initialized in parameter space $\left[0.5 \vartheta^{*}, 1.5 \vartheta^{*}\right]$, where $\vartheta^{*}$ is the vector of nominal, reference parameter values used to generate the artificial noisy measurements. The initial number of feasible parameter vectors required was $n\left(\Omega_{\vartheta_{F}, i n i}\right)=15$. Sampling size for each iteration was: $n\left(S_{\theta, \text { in }}\right)=n\left(S_{\theta, \text { out }}\right)=50$. Details for each test case are provided in Table 4.1. See the Appendix of this chapter for a list of symbols and parameter values for each model. 
TABLE 4.1: Description of the test model cases.

\begin{tabular}{lccc}
\hline Case & $\begin{array}{c}\text { Bounded } \\
\text { states }\end{array}$ & $\begin{array}{c}\text { Estimated } \\
\text { parameters }\end{array}$ & $W D_{\text {tol }}$ \\
\hline (1) Lotka-Volterra & $x_{1}$ & $p_{2}, p_{4}$ & 0.005 \\
(2) Lotka-Volterra & $x_{1}, x_{2}$ & $p_{2}, p_{4}$ & 0.005 \\
(3) Monod & $X$ & $\mu_{\text {max }}, K_{S}, Y, k_{d}$ & 0.5 \\
(4) GRASIM & $W_{D M}$ & $a, \beta, k, \phi$ & 0.5 \\
\hline
\end{tabular}

\section{Lotka-Volterra}

The Lotka-Volterra model is a canonical biological model that describes the dynamics of predator-prey populations. It is represented by the differential equations:

$$
\begin{aligned}
& \dot{x_{1}}=x_{1}\left(p_{1}-p_{2} x_{2}\right) \\
& \dot{x_{2}}=-x_{2}\left(p_{3}-p_{4} x_{1}\right)
\end{aligned}
$$

where the states are prey $\left(x_{1}\right)$ and predator $\left(x_{2}\right)$ populations, and the four parameters $(p)$ represent rates of population change due to growth, death, and predation. This model has been used previously to test algorithms for set-membership parameter estimation (Marvel and Williams, 2012; Raissi et al., 2004).

We used the Lotka-Volterra model to analyse the algorithm behaviour on two identifiable cases: bounding one state $\left(x_{1}\right)$ and two states $\left(x_{1}, x_{2}\right)$.

\section{Growth with Monod kinetics}

Microbial growth and degradation with Monod kinetics is represented by the differential equations:

$$
\begin{aligned}
\dot{S} & =-\mu_{\max } \frac{X}{Y} \frac{S}{K_{S}+S} \\
\dot{X} & =\mu_{\max } \frac{S}{K_{S}+S} X-k_{d} X
\end{aligned}
$$

where the states are concentrations of substrate $S$ and bacteria $X$, with interactions described by four parameters related to growth, decay, yield efficiency, and substrate limitation.

The Monod kinetics is theoretically identifiable from noise-free measurements, but its parameters cannot be uniquely identified from noisy measurements (Holmberg, 1982). Therefore, we used this model to analyse the algorithm behaviour on one unidentifiable case. 


\section{GRASIM}

The grass growth model in GRASIM splits the plant into components for storage and structure (Mohtar et al., 1997):

$$
\begin{aligned}
& \dot{W}_{S}=f_{P}-f_{S R}-f_{G}-f_{M R} \\
& \dot{W}_{g}=f_{G}-f_{S} \\
& W_{D M}=W_{g} / 0.4
\end{aligned}
$$

with carbon mass flows for photosynthesis $\left(f_{P}\right)$, shoot respiration $\left(f_{S R}\right)$, structure growth $\left(f_{G}\right)$, maintenance respiration $\left(f_{M R}\right)$, and senescence $\left(f_{S}\right)$. The model output $\left(W_{D M}\right)$ is the dry matter of plant structure mass, assuming $40 \% \mathrm{C}$ content in dry matter. See the Appendix of this chapter for the detailed model.

We used the GRASIM model to test the applicability of our algorithm on a larger model, aiming towards implementation in biological and environmental systems.

Commonly, estimating multiple parameters in one model is preceded by a sensitivity analysis, to find the most dominant parameters. If however, more than four dominant parameters are found, the computational complexity of the algorithm will further increase for a given $W D_{t o l}$ and FPS coverage. Consequently, for higher dimensional parameter cases, accuracy of the algorithm can be relaxed by increasing $W D_{\text {tol }}$ and $N_{\text {ini }}$, ultimately leading to the MCSM algorithm by Keesman and Van Straten (1990), demonstrated on a 16-dimensional parameter case by Van Straten and Keesman (1991).

\subsection{Results and discussion}

The main results for each case are shown in Table 4.2. Due to the sample-based nature of the algorithm, results varied between runs, but the behaviour and final accuracy remained consistent.

The choice of $W D_{t o l}$ is arbitrary. It depends on previous experience with the model and on the desired accuracy.

Notice that we assumed an error of fixed width for all artificial measurement instants, which constitutes a worst-case scenario for underestimation $(U E)$.

It is important to note that in spite of the geometrical relation, the proposed characterisation by spheres is different from the characterisation by ellipsoids. The spheres proposed here are solely based on the Voronoi tessellation of $\theta_{U}$, and do not provide a direct description of the direction or shape of the approximate FPS. In contrast, ellipsoids use tools like principal component analysis (PCA) of $\theta_{F}$ to describe the directionality of the approximate FPS. 
TABLE 4.2: Results (median) from five runs of the algorithm on four cases: number of spheres $(n(s p h))$, overestimation $(O E)$, underestimation $(U E)$, weighted deviation $(W D)$, number of iterations $(n(i t))$ and computational time $\left(t_{\text {comp }}\right)$. Case 3 did not converge at the given $W D_{t o l}$. Case 4 includes results with and without convergence.

Notice that $t_{\text {comp }}$ is proportional to the number of iterations $(O(n))$ for this sample-based method, as opposed to $O(n)^{p}$ for interval-based methods.

\begin{tabular}{lcccccc}
\hline Case & $n(s p h)$ & $O E$ & $U E$ & $W D$ & $n($ it $)$ & $t_{\text {comp }}[s]$ \\
\hline$(1) \mathrm{L}-\mathrm{V}$ & 8 & $3.5 \times 10^{-2}$ & 1.3 & $2.9 \times 10^{-3}$ & 9 & 2.7 \\
$(2) \mathrm{L}-\mathrm{V}$ & 9 & $6.8 \times 10^{-3}$ & 2.8 & $3.6 \times 10^{-4}$ & 12 & 2.8 \\
(3) $\mathrm{M}$ & 330 & 1.3 & 15 & 11 & 70 & 352 \\
(4) G & 515 & 2.2 & 1.8 & 0.9 & 94 & 2490 \\
\hline
\end{tabular}

\section{Case 1) Lotka-Volterra.}

\section{1 bounded output and 2 estimated parameters.}

For case 1 , we assumed that only $x_{1}$ was measured and bounded $\left(y=x_{1}\right)$. The approximate FPS was characterised by a maximum of 10 spheres (Fig. 4.5.1). The algorithm converged after a maximum of 14 iterations (1400 model simulations). Deviations between FMOS and error bounds show a negligible OE (Fig. 4.5.2). However, the convergence history shows a stagnant underestimation (Fig. 4.5.3), which means that the model cannot reproduce the entire error bounds (which in general is the case). Nonetheless, WD converged because there were no new $\theta_{F}$ found outside the sphere set in the last iteration.

Uncertainty propagation from $\Omega_{\theta_{F}}$ on $x_{2}$ (Fig. 4.5.4) is similar to the one obtained by the SIVIA algorithm (Marvel and Williams, 2012). However, due to random sampling, any $\vartheta_{U}$ in the approximate FPS could introduce a deviation in $x_{2}$ beyond the propagation from $\vartheta_{F}$. This deviation and the uncertainty propagation itself can be avoided by bounding $x_{2}$. We show this in the next case. 
1)

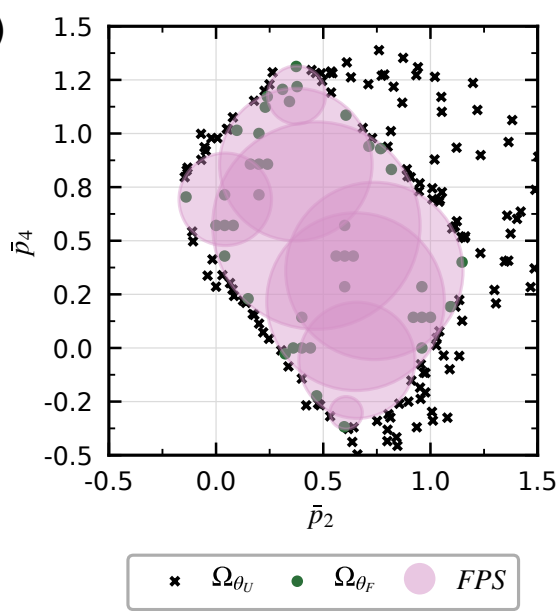

3)

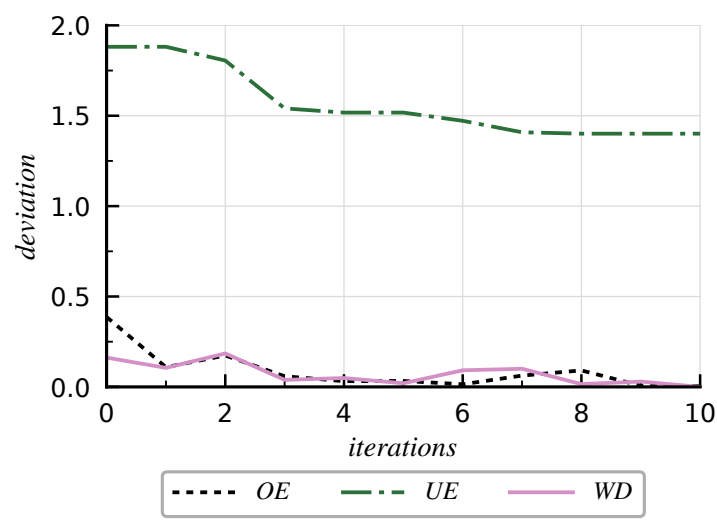

2)

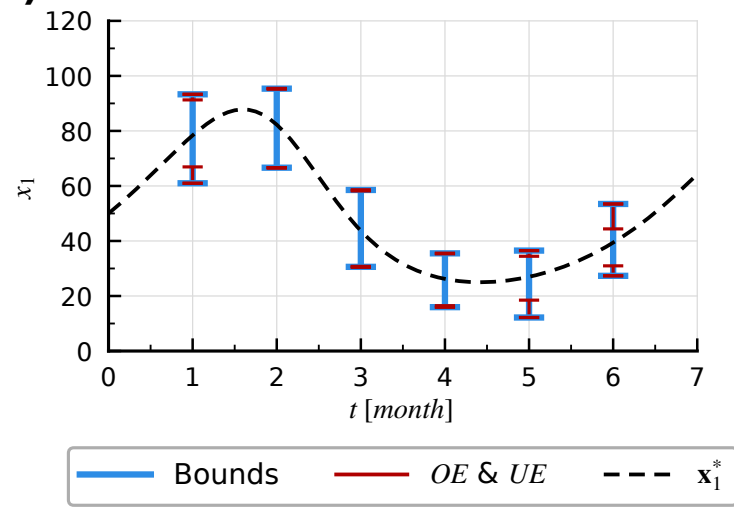

4)

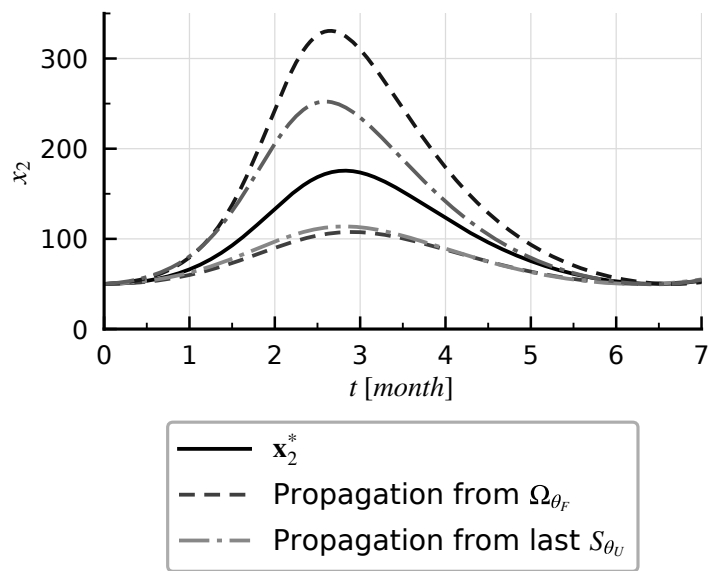

FIGURE 4.5: Case 1. Lotka-Volterra with 1 bounded output and 2 estimated parameters. Example results from one algorithm run. 1) Approximate FPS.

Normalized $\mathbb{R}^{2}\left(\bar{p}_{2}, \bar{p}_{4}\right)$ from $p_{2} \in[0.007,0.012]$ and $p_{4} \in[0.019,0.022]$. 2) Reference model output, overestimation $(O E)$ and underestimation $(U E) . O E$ is negligible. $U E$ in

the first and last two measurement instants indicate that the model cannot fully reproduce the data error bounds. 3) Convergence. UE stays high but convergence is reached because few or no new $\theta_{F}$ were found in the last iterations, reducing $W D$.

4) Uncertainty propagation on $x_{2}$ from the approximate FPS.

\section{Case 2) Lotka-Volterra.}

\section{2 bounded outputs and 2 estimated parameters.}

For case 2, we assumed error bounds on both states $\left(y=\left[x_{1}, x_{2}\right]^{T}\right)$. The approximate FPS was characterised by a maximum of 11 spheres (Fig. 4.6.1). The algorithm converged after a maximum of 15 iterations, for a total of 1500 model simulations.

When bounding two states, there is a reduced degree of freedom with respect to case 1, which results in a FMOS with smaller intervals at each measurement instant $k$, and thus larger $U E$ (Figs. 4.6.2). However, as expected, the bounds on $x_{2}$ led to a smaller $O E$ on this state compared to case 1 . 
1)

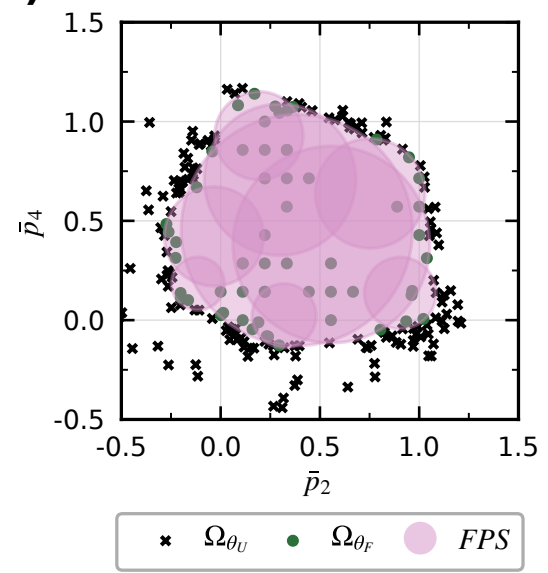

2)
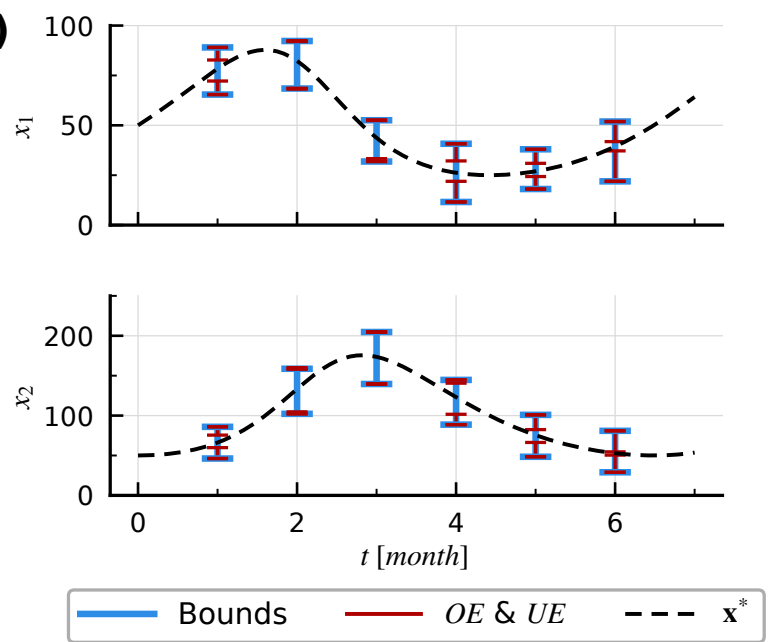

FIGURE 4.6: Case 2) Lotka-Volterra with 2 bounded outputs and 2 estimated parameters. Example result from one algorithm run. 1) Approximate FPS. Normalized $\mathbb{R}^{2}\left(\bar{p}_{2}, \bar{p}_{4}\right)$ from $p_{2} \in[0.009,0.011]$ and $p_{4} \in[0.019,0.022]$.

2) Reference model output, overestimation $(O E)$ and underestimation $(U E)$.

Compared to case 1, the FMOS covers a lower range between the bounds in $x_{1}$

(higher $U E$ ), but uncertainty propagation on $x_{2}$ decreases to a negligible $O E$

\section{Case 3) Biodegradation and growth with Monod kinetics.}

\section{1 bounded output and 4 estimated parameters. Unidentifiability.}

We tested the algorithm under a well-known case of an unidentifiable model structure. We assumed that only bacterial biomass was measured (bounded for model output $y=X$ ). The estimation of all four parameters in the Monod kinetics model resulted in an approximate FPS that extends indefinitely with subsequent iterations (Fig. 4.7.1). The projection of parameter combination $\left(\bar{\mu}_{\text {max }}, \bar{K}_{S}\right)$ shows an elongated set, because estimates of these parameters are highly correlated. This result is in accordance with Holmberg (1982). Similarly, the projection of parameter combination $\left(\bar{Y}, \bar{k}_{d}\right)$ also shows an elongated set, indicating unidentifiability. Practical unidentifiability of this model is also reflected in high $O E$ peaks, resulting from continuously finding new areas with $\theta_{F}$ that must be reshaped to exclude $\theta_{U}$ (Figs. 4.7.2 and 4.7.3).

Therefore, high peaks of $O E$ through convergence history combined with a FPS that extends far beyond the normalized coordinates $[0,1]$ constitute a novel test for practical identifiability. These peaks also illustrate the opportunity to improve the mathematical rigour of the algorithm by developing a function to describe the convergence trend, for example by a moving average filter of the historical $O E$ and $U E$. 
1)

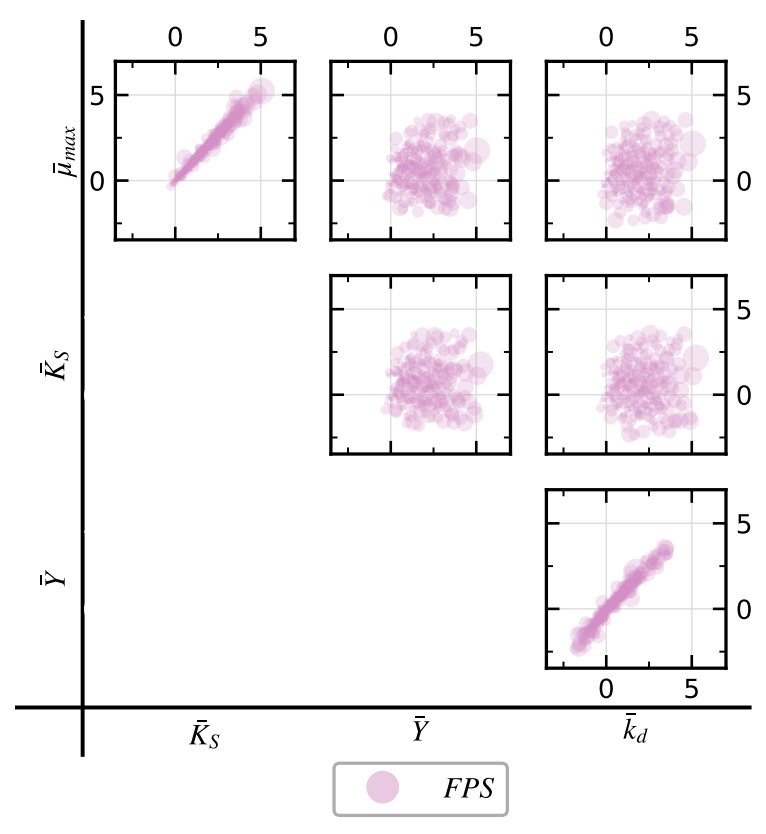

2)

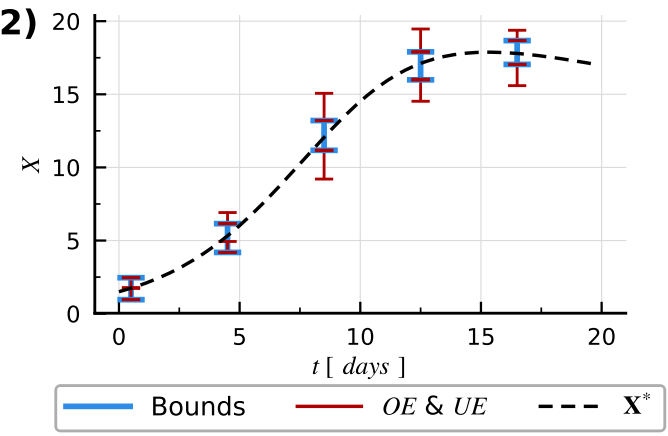

3)

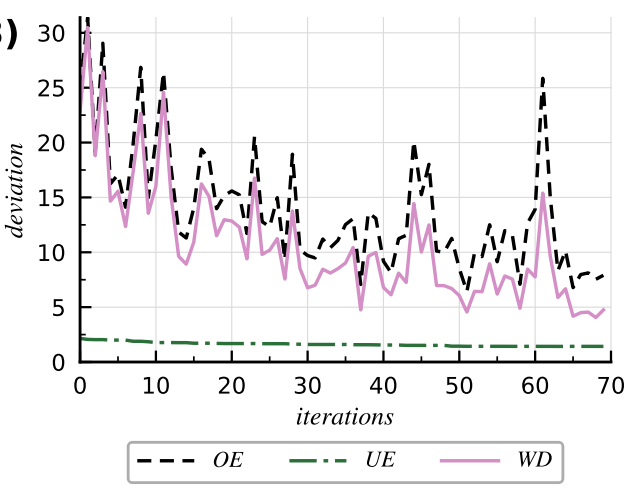

FIGURE 4.7: Case 3) Microbial growth with Monod kinetics, with 1 bounded output and 4 estimated parameters. Example result from one algorithm run after 70 iterations. Unidentifiable model. 1) Approximate FPS. ( $\mathbb{R}^{2}$ projections from $\mathbb{R}^{4}$ space). Normalized parameter space $\left(\bar{\mu}_{\text {max }}, \bar{K}_{S}, \bar{Y}, \bar{k}_{d}\right)$ from $\mu_{\max } \in[0.79,1.49]$,

$K_{S} \in[3.09,6.87], Y \in[9.60,11.07], k_{d} \in\left[1.41 \times 10^{-2}, 2.71 \times 10^{-2}\right]$. Due to unidentifiability of the model structure, the approximate FPS continues to extend with every subsequent iteration far beyond the normalized coordinates $[0,1]$.

2) Reference model output, overestimation $(O E)$ and underestimation $(U E)$. Compared to the previous cases, $O E$ cannot be neglected. 3) Convergence. After 50 iterations, $O E$ and weighted deviation $(W D)$ stop decreasing because new $\theta_{U}$ and $\theta_{F}$ continue to be found with every subsequent iteration. The approximate FPS is thus not bounded, reflecting model unidentifiability.

\section{Case 4) Crop growth model.}

\section{1 bounded output and 4 estimated parameters.}

Finally, we tested the algorithm on a model with higher complexity, GRASIM. Steps often trivialized prior to calibration of complex models are a sensitivity analysis and the implementation of prior knowledge (parameter constraints). We performed a Sobol global sensitivity analysis on the model (Sobol, 2001) using the Python package SALib (Herman and Usher, 2017). The sensitivity analysis revealed that six out of the ten model parameters have a global sensitivity coefficient at least one order of magnitude larger than the rest. From those six parameters, a combination of four 
parameters is chosen with the lowest second-order sensitivity coefficients (reflecting low correlation in the parameter estimates). This pre-selection helps improving model identifiability. The selected parameters were: structural specific leaf area $(a)$, senescence rate $(\beta)$, extinction coefficient of canopy $(k)$, and photosynthetic fraction available for growth $(\phi)$.

Subsequently, we defined parameter constraints for the algorithm based on physical definitions and on reported ranges in literature (Mohtar et al., 1997; Zhai et al., 2004). These constraints limit the region where spheres will characterise the approximate FPS: $a \in[0.002,0.008], \beta \in[0.02,0.1] k \in[0.25,0.90]$, and $\phi \in[0.75,0.99]$.

In this case, simulation time of the model governs computational time in the algorithm implementation (ca. $2 \mathrm{~min}$ per iteration). Therefore, we set maximum iterations to 100 . The algorithm converged to $W D_{t o l}=0.5$ in 2 out of 5 runs. In case of convergence, the approximate FPS was characterised by a maximum of 430 spheres in 88 iterations.

The approximate FPS is bounded within the constraints provided (Fig. 4.8.1). Notice that the projection of $(a, k)$ is a non-convex set, in a narrow space, which can still be characterised by the algorithm, but requires a relatively high number of spheres. This projection also indicates a hyperbolic (inversely proportional) relationship between $a$ and $k$ : higher values for specific leaf area $(a)$ indicate thinner grass leaves, which result in lower values for canopy extinction of light $(k)$. Notice a visible $O E$ at $t_{k}$ equal to 150 and $180 d$ (Fig. 4.8.2), when growth rates are maximum. Parameters $a, k, \phi$ are directly related to growth rate, and small changes in their values impact model output. It may be possible to decrease $O E$ by defining submodels for different time ranges (growth stages), thus introducing a time-varying FPS. However, this study focuses on the characterisation of a time-invariant FPS.

The model itself appears to be unidentifiable for the four parameters chosen, but the implementation of constraints based on the physical meaning of parameters prevents an unbounded FPS.

This case shows the relevance of a sensitivity analysis prior to parameter estimation. A similar estimation of 3 parameters for this model, resulted in a similar $O E$ after convergence to $W D<0.5$. Therefore, adding parameters for estimation may require more computational time and more memory, with little improvement in the FMOS accuracy, and may introduce unidentifiability to the model. 
1)

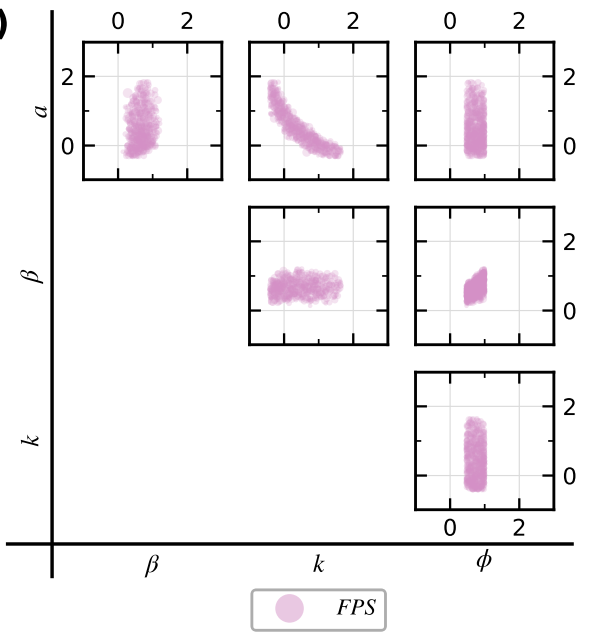

2)

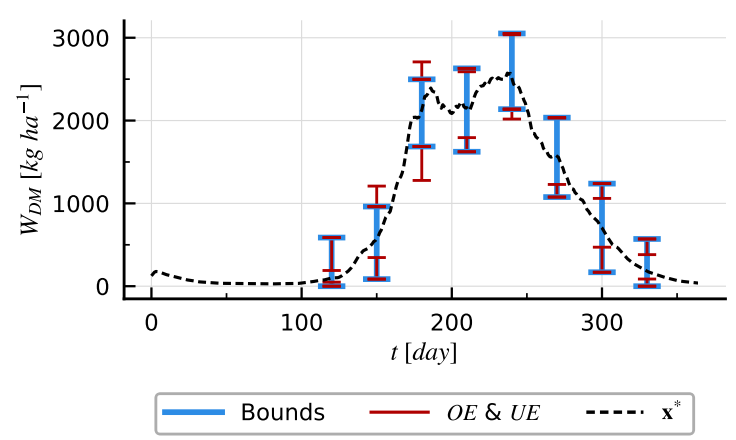

FIGURE 4.8: Case 4) Grass growth in GRASIM model, with 1 state bounded and 4 estimated parameters. Example result from one algorithm run. 1) Approximate FPS. ( $\mathbb{R}^{2}$ projections from $\mathbb{R}^{4}$ space). Normalized parameter space $(\bar{a}, \bar{\beta}, \bar{K}, \bar{\phi})$ from $a \in[0.0030,0.0056], \beta \in[0.027,0.061], k \in[0.394,0.688], \phi \in[0.614,0.963]$. Although unidentifiable, an approximate FPS can be characterised due to the constraints on parameter values based on literature and their physical meaning. 2) Reference model output, overestimation $(O E)$ and underestimation $(U E)$. At $t_{k}=150,180 d$, when growth rates are higher, $O E$ becomes larger.

\subsection{Conclusions}

Our objective was to investigate and evaluate an efficient sampling-based method for set-membership parameter estimation. We presented a set-valued algorithm that characterises an approximate feasible parameter set (FPS) of (hyper)spheres, and provides a balance between accuracy and computational complexity. The spheres were obtained using randomly sampled unfeasible parameter vectors as seeds for a Voronoi diagram of the parameter space, and using the resulting Voronoi vertices as centres for the spheres. The algorithm works with a fixed number of sampled parameter vectors per iteration, i.e. the computational time has an order $O(n$ (sample $))$. Although lacking an exact mathematical description, the approximate FPS can generally be characterised by a low number of spheres.

We demonstrated an implementation on a 2-parameter case, achieving lower computational requirements and similar accuracy, as compared with the SIVIA algorithm. We also demonstrated implementation on a 4-parameter case of a relatively complex model.

Additionally, we proposed a measure of the accuracy based on deviations between feasible model output set (FMOS) and data error bounds. When analysing both FPS and these deviations, it is possible to indicate practical identifiability of a model, as we demonstrated on a 4-parameter case. 


\subsection{Appendix}

\section{Supporting functions}

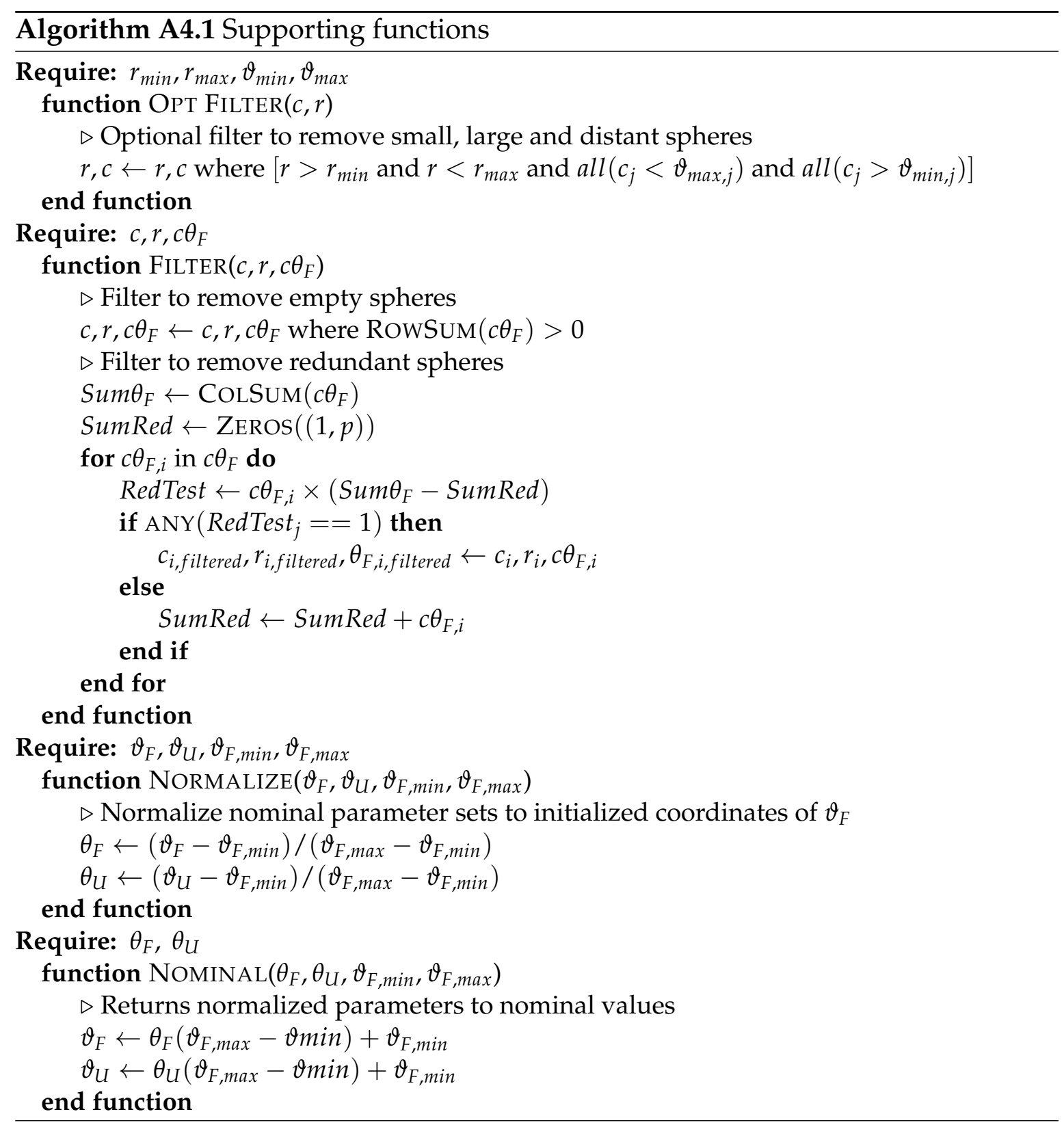




\section{List of symbols}

TABLE A4.1: Symbols used in the algorithm.

\begin{tabular}{ll}
\hline Symbol & Description \\
\hline$\|e\|_{\infty}$ & $\infty$-norm of the error vector, $\|e\|_{\infty}:=\max _{1 \leq i \leq n}\left|e_{i}\right|$ \\
$F P S$ and FMOS & Feasible parameter set, and feasible model output set \\
$k, M$ & Measurement instant, and total of measurement instants \\
$n()$ & Cardinality of a set (number of elements) \\
$O()$ & Order of the algorithm (time complexity) \\
$O E, U E, W D$ & Overestimation, underestimation and weighted deviation \\
$\mathbb{R}^{p}$ & -dimensional parameter space \\
$S_{\vartheta}$ & Sample of parameter vectors \\
$\varepsilon$ & Upper error bound \\
$\vartheta,(\theta)-F$ or $U$ & Parameter vector (normalized). Feasible or unfeasible \\
$\Omega_{\vartheta},\left(\Omega_{\theta}\right)$ & Set of all known parameter vectors (normalized) \\
\hline
\end{tabular}

\section{Model parameters}

TABLE A4.2: Model parameters and their reference values for each case.

\begin{tabular}{|c|c|}
\hline \multicolumn{2}{|l|}{ Lotka-Volterra model } \\
\hline$x_{1}, x_{2}(50,50)$ & State variables (initial value), preys and predators \\
\hline$t_{\text {sim }}=[0,1, \ldots, 7 * 30]$ day & Simulation time \\
\hline$t_{k}=[1,2, \ldots, 6]$ month & Artificial measurement instants \\
\hline$x_{1}^{*}, x_{2}^{*}$ & Reference model outputs \\
\hline Parameters (ref. value) & \\
\hline $\bar{p}_{2}, \bar{p}_{4}$ & Estimated, normalized parameters \\
\hline$p_{1}\left(\mathbf{p}_{1}^{*}=1.0\right.$ month $\left.^{-1}\right)$ & Birth rate of prey \\
\hline$p_{2}\left(\mathbf{p}_{2}^{*}=0.01\right.$ month $^{-1}$ pred. $\left.^{-1}\right)$ & Population decrease of prey due to predators \\
\hline$p_{3}\left(\mathbf{p}_{3}^{*}=1.0\right.$ month $\left.^{-1}\right)$ & Predator death rate \\
\hline$p_{4}\left(\mathbf{p}_{4}^{*}=0.02\right.$ month $^{-1}$ prey $\left.^{-1}\right)$ & Population increase of predators due to prey \\
\hline \multicolumn{2}{|l|}{ Microbial growth model } \\
\hline$[S, X](2.0,1.5) \mathrm{mg} / \mathrm{L}$ & State variables (initial value), substrate and bacteria \\
\hline$t_{\text {sim }}=[0,1 / 24, \ldots, 20] d$ & Simulation time \\
\hline$t_{k}=[0.5,3.5, \ldots, 16.5] \mathrm{hr}$ & Artificial measurement instants \\
\hline $\mathbf{S}^{*}, \mathbf{X}^{*}$ & Reference model outputs \\
\hline \multicolumn{2}{|l|}{ Parameters (ref. value) } \\
\hline $\bar{k}_{d}, \bar{K}_{S}, \bar{Y}, \bar{\mu}_{\max }$ & Estimated, normalized parameters \\
\hline$k_{d}\left(\mathbf{k}_{\mathbf{d}}^{*}=0.021 d^{-1}\right)$ & Endogenous bacterial decay \\
\hline$K_{S}\left(\mathbf{K}_{S}^{*}=5.14 m g L^{-1}\right)$ & Half-velocity constant \\
\hline$Y\left(\mathbf{Y}^{*}=10.34 m g_{X} m g_{S}^{-1}\right)$ & True microbial yield per substrate consumed \\
\hline$\mu_{\max }\left(\stackrel{-\max }{*}=1.15 d^{-1}\right)$ & Maximum rate of substrate use \\
\hline
\end{tabular}


TABLE A4.3: Model parameters and their reference values for each case (continued).

\begin{tabular}{ll}
\hline GRASIM model & \\
\hline$\left[W_{s}, W_{g}\right](50,50) \mathrm{kgC} \mathrm{ha}^{-1}$ & State variables (initial value), storage and structure mass \\
$t_{s i m}=[0,1, \ldots, 365] d$ & Simulation time \\
$t_{k}=[120,150, \ldots, 330] \mathrm{hr}$ & Artificial measurement instants \\
$\mathbf{W}_{\mathrm{DM}}^{*} \mathrm{kgDM} h \mathrm{ha}^{-1}$ & Reference model output, dry mass of plant \\
Parameters (ref. value) & \\
$\bar{a}, \bar{\beta}, \bar{k}, \bar{Y}$ & Estimated, normalized parameters \\
$a\left(\mathbf{a}^{*}=0.004 h a \mathrm{~kg}^{-1}\right)$ & Structural specific leaf area \\
$\alpha\left(\boldsymbol{\alpha}^{*}=2.4 \times 10^{-9} \mathrm{~kg} \mathrm{~J}^{-1}\right)$ & Leaf photosynthetic efficiency \\
$\beta\left(\boldsymbol{\beta}^{*}=0.05\right)$ & Senescence rate \\
$k\left(\boldsymbol{k}^{*}=0.5\right)$ & Canopy extinction coefficient \\
$m\left(\boldsymbol{m}^{*}=0.1\right)$ & Leaf transmission coefficient \\
$\mu_{m}\left(\boldsymbol{\mu}_{m}^{*}=0.5 d^{-1}\right)$ & Max. structural specific growth rate \\
$\phi\left(\boldsymbol{\phi}^{*}=0.9\right)$ & Photosynthetic fraction available for growth \\
$P_{m}\left(\mathbf{P}_{\mathbf{m}}^{*}=10000 \mathrm{~kg} \mathrm{ha}^{-1} d^{-1}\right)$ & Max. photosynthesis \\
$Y\left(\mathbf{Y}^{*}=0.75\right)$ & Structure fraction from storage \\
$M\left(\mathbf{M}^{*}=0.02 d^{-1}\right)$ & Maintenance respiration coefficient \\
\hline
\end{tabular}

\section{Grasim auxiliary equations}

Photosynthetic growth is defined as:

$$
f_{P}=(12 / 44) \phi P
$$

where $(12 / 44)$ is a conversion factor for mass from $\mathrm{CO}_{2}$ to $C$.

The photosynthesis rate is defined as:

$$
\begin{aligned}
P & =\int_{0}^{L A I} \int_{0}^{h} P g \mathrm{~d} l \mathrm{~d} \tau \\
P g & =\frac{\alpha I P_{m}}{\alpha I+P_{m}} \\
I & =\frac{k}{1-m} I_{0} e^{-k l}
\end{aligned}
$$

where $L A I$ is the leaf area index, $h$ is the daytime duration, $P_{g}$ is the gross photosynthetic rate, $I$ is the light intensity over a leaf, and $I_{0}$ is the irradiance.

Assuming $h=24 h r$ and $I_{0}=D L I$ (day light integral), it is possible to obtain an approximate analytical solution for $P$ :

$$
\begin{aligned}
P & =\frac{P_{m}}{k} \ln \left(\frac{A+P m}{A e^{-k * L A I}+P m}\right) \\
A & =\alpha D L I \frac{k}{1-m}
\end{aligned}
$$

where DLI for the test case was taken from the weather station De Bilt for year 2001 (KNMI, 2014). 
The leaf area index is:

$$
L A I=a W_{g}
$$

The net mass conversion from storage to structure is:

$$
f_{G}=\mu_{m} \frac{W_{g} W_{s}}{W_{g}+W_{s}}
$$

Shoot respiration from storage mass is:

$$
f_{S R}=\mu_{m} \frac{1-Y}{Y} \frac{W_{g} W_{s}}{W_{g}+W_{S}}
$$

Maintenance respiration (covered entirely by storage mass) is:

$$
f_{M R}=M W_{g}
$$

Senescence is:

$$
f_{S}=\beta W_{g}
$$





\title{
Chapter 5
}

\section{Set-membership uncertainty assessment of a mathematical model for nutrient balances in fish growth and excretions.}

\begin{abstract}
Adapted and submitted for publication as:
Daniel Reyes Lastiri, Jan-David Wacker, Simon van Mourik \& Karel J. Keesman, "Set-membership uncertainty assessment of a mathematical model for nutrient balances in fish growth and excretions". In journal: Aquacultural Engineering.
\end{abstract}

\section{Abstract}

Waste streams from inland aquaculture contain nutrients that could be further used. Knowledge about concentration dynamics of nutrients throughout fish growth can help designing and operating technologies for nutrient reuse and recovery. Live insitu monitoring is costly and uncommon in commercial applications. Mathematical models based on experimental measurements can provide the required knowledge on nutrient dynamics. However, model identification is hindered by measurement uncertainties, common in aquaculture systems.

We investigated the dynamics and uncertainties in fish growth and nutrient excretions. For this purpose, we developed a mathematical model describing nutrient balances in fish, using only four model parameters. The model was based on simplification and semi-physical approaches, to facilitate model parameter estimation. The model was calibrated considering multiple time scales and using the setmembership approach, suitable for parameter estimation under bounded measurement uncertainties, and aimed at describing parameter uncertainty in terms of a set rather than point estimates with confidence intervals. Propagation of bounded model prediction uncertainties was assessed using set-membership Monte Carlo 
simulations. From the experimental measurements reported in literature, the model prediction uncertainty explained $84 \%$ of the data variability for fish growth, $75 \%$ for digestive system content, and $28 \%$ for ammonia excretion rate. The limited coverage of ammonia excretion rate variability was explained by the use of controlled experimental conditions that affect excretions in feedback loops between water and fish. The largest prediction uncertainties were observed in soluble excretion of $P$, $\mathrm{Ca}$, and $\mathrm{Na}$. For improved prediction accuracy, future experimental measurements should focus on these nutrients, using common growth conditions.

\subsection{Introduction}

Fish and crustaceans have a high protein production efficiency (Brown, 2001), and a low carbon footprint (FAO, 2018b), thus presenting a sustainable option for the protein transition, moving away from conventional meat production and capture fisheries. Aquaculture has shown a continuous development since the mid 20th century, accounting for 47\% of global fish production in 2016 (FAO, 2018b). This development has helped in decreasing capture production and its impacts. Further developments in aquaculture should aim at decreasing impact on biodiversity and aquatic ecosystems, following the targets for life below water (14.1), and biodiversity and ecosystem (15.1) of the UN Sustainable Development Goals (UN, 2015). One of the challenges in aquaculture is the reduction of waste discharge. To address this challenge, intensive inland production methods such as recirculating aquaculture systems (RAS), apply technologies for waste treatment and reduction to improve water quality in the fish tanks, decreasing the direct environmental impacts of aquaculture. These technologies include, for example, mechanical filtration of solids and biofiltration to convert ammonia into nitrate. However, waste streams from inland aquaculture, are still rich in nutrients that could be further utilised (Timmons and Ebeling, 2010). Knowledge of nutrient concentrations and dynamics is required for the design and operation of technologies for nutrient reuse and recovery. Therefore, improved understanding of nutrient dynamics in waste streams presents opportunities for higher resource efficiency of inland aquaculture.

Waste from inland aquaculture in RAS consists of a solids stream with approximately $20 \%$ of the phosphorus eaten by fish, and a dissolved stream with $60-90 \%$ of the nitrogen eaten by fish (Timmons and Ebeling, 2010). Nitrogen can be lost via denitrification when anaerobic zones are present (Reyes Lastiri et al., 2018). Therefore, additional technologies for nutrient treatment and recovery have been proposed. Solids can be further treated by thickening and stabilization to prevent denitrification losses, and facilitate transport and application as fertilizer. Thickening can be accomplished with sedimentation, textile bags, belt filters, and drying beds 
(Cripps and Bergheim, 2000; Timmons and Ebeling, 2010); and stabilization can be accomplished by lime addition and composting (Chen et al., 1997; Timmons and Ebeling, 2010). Similarly, options for direct nutrient reuse have also been proposed. For example, dissolved nutrients can be reused as hydroponic fertilizer in aquaponic systems (aquaculture + hydroponics = aquaponics). In particular, theoretical studies in aquaponics have proposed the use of an UASB reactor for phosphorus remineralization of the solids stream (Goddek et al., 2016), and reverse osmosis to concentrate the dissolved stream (Goddek and Keesman, 2018).

The main challenge in the application of the aforementioned management and reuse strategies is the identification of nutrients in waste streams. For example, composting of solids requires knowledge on the carbon to nitrogen ratio (C:N) (Timmons and Ebeling, 2010), and aquaponics requires knowledge about concentrations of macronutrients from fish that could be harmful to plants, such as $\mathrm{Na}$, which limit the potential reuse of other macronutrients (Reyes Lastiri et al., 2018). Due to the dynamic nature of fish growth and excretions, nutrient concentrations in waste streams are highly dynamic. Detailed real-time information about the dynamics of these nutrients would require continuous sensing. However, membrane-based sensors commonly used for this purpose are prone to errors from clogging, and require frequent replacement, resulting in additional costs. Alternatively, laboratory measurements are more accurate but slower and similarly expensive. Continuous sensing is therefore uncommon in commercial applications and still limited to research (Timmons and Ebeling, 2010; Su et al., 2020). It is worth noting that emerging technologies based on optoelectronics may help solving the sensing challenges in aquaculture (Fritzsche et al., 2018).

Monitoring nutrient dynamics from inland aquaculture can be improved by data assimilation, i.e. complementing in-situ live measurements with mathematical models, starting from the dynamics of fish excretions. Several models for fish growth are available in literature. However, they are not directly applicable for nutrient reuse, as they focus on productivity, and not on nutrient dynamics.

Simple models, such as linear and quadratic models, have been used to study fish growth and response to nutrient intake (Belal, 2005). However, these models are based on parameters without a biological meaning, and they focus on a narrow time scale, limiting their applicability to single cases. Saturation kinetic growth models introduce parameters with biological meaning, broadening applicability. However, neither of these simple models describe nutrient uptake and excretions. Other empirical models based on (variable) feed conversion ratio, coupled with steady-state mass balances, have been used to predict nutrient excretions for the design of RAS and aquaponic systems (Timmons and Ebeling, 2010; Reyes Lastiri et al., 2016), but they do not describe excretion dynamics. 
More complex examples, like bioenergetic models, reflect the dynamics of fish growth based on model parameters with a biological meaning (see for example Yi (1998) and Van Trung et al. (2011)), but they do not describe nutrient uptake or excretions. Furthermore, past implementations of bioenergetic models for fish growth frequently overlooked conservation laws (Canale and Breck, 2013). Similarly complex, the Dynamic Energy Budget (DEB) model (Kooijman and Kooijman, 2010) has been applied to fish in aquaculture (Serpa et al., 2013), but the model calibration used a one-at-a-time sensitivity analysis, which does not reflect the parameter interactions in the model structure. These physiological models may appeal to the modeller because they reflect detailed processes and could, in principle, provide better understanding of the system. However, these model structures typically contain more than 10 parameters, which easily lead to parameter estimation problems like local minima and strong correlations between estimates.

Alternatively, semi-physical or grey box models have also been proposed, which provide a balance between simplicity and detailed descriptions. For example, Wik et al. (2009) developed an integrated RAS model, where fish excretions were split into particulate and soluble fractions for nitrogen and phosphorus, based on the Activated Sludge Model (ASM) approach for municipal wastewater. Similarly, Reyes Lastiri et al. (2018) developed an ASM-based model for several macronutrients from fish and their application in aquaponics. Both studies combine a semi-empirical model for fish growth with nutrient balances, but they are not yet verified against experimental data.

One of the challenges in model verification against experimental data, for fish growth and excretions, is the presence of multiple time scales. Fish growth dynamics spans several months, whereas excretions dynamics spans several hours. One possible solution is the use of experimental measurements reported in multiple literature sources. However, this approach introduces unknown or uncertain measurement conditions.

Recently, quantifying uncertainties in decision support models has gained attention. Uusitalo et al. (2015) recommend to provide a transparent representation of these uncertainties on each level of a model. However, to the best of our knowledge, uncertainty studies for fish nutrient uptake and excretions in aquaculture have not been performed. Furthermore, experimental data on nutrient flows may be available in literature, but detailed measurement conditions are rarely reported, introducing modelling and sampling errors. One approach suitable for model development under limited data, correlated measurement uncertainties, and modelling error, common in aquaculture systems, is the set-membership approach (Norton, 1987; Keesman, 1990; Van Straten and Keesman, 1991; Walter and Piet-Lahanier, 
1990; Milanese et al., 1996). In the past decade, set-membership gained renewed interest in modelling studies (Rumschinski et al., 2010; Diniz, 2013; Cerone et al., 2014; Nurulhuda et al., 2017).

In summary:

1. Waste streams from inland aquaculture contain nutrients that could be further utilised.

2. Technologies for nutrient reuse and recovery require knowledge about nutrient dynamics throughout the fish growth cycle.

3. Semi-physical models provide suitable knowledge basis, combining a simplified mathematical description with in-situ and experimental measurements, facilitating model identification and parameter estimation.

4. The semi-physical model should have a small number of parameters, describe the essential dynamics and time scales, and account for prediction uncertainties.

The objective of this study was to investigate dynamics and uncertainties of macronutrients in inland aquacultural wastewater, to aid in the design of nutrient reuse and recovery technologies. For this purpose, a semi-physical model for nutrient dynamics was developed, describing fish growth and excretion processes based on mass balances. The model development followed a model simplification approach, to maintain minimum complexity and to facilitate the estimation of model parameters and their uncertainties. The model was calibrated using a setmembership approach, suitable for the limited and uncertain data that was available. Finally, an analysis of the prediction errors and the uncertainty propagation was used to identify suggestions for future experimental conditions and measurements.

\subsection{Methods}

The mathematical model was aimed at understanding the fish excretion process in inland aquaculture systems. The model focuses on the production of Nile tilapia (Oreochromis niloticus), due to the increasing availability of empirical knowledge and data from inland aquaculture production and research.

The mathematical model was based on system dynamics, semi-physical, and model simplification approaches, maintaining low complexity and therefore facilitating identifiability, such that, in principle, it would be possible to uniquely estimate the unknown model parameters.

The model describes nutrient balances using a system of differential equations with supporting algebraic equations, splitting waste streams into particulate matter 
(fish faeces), and soluble matter (generalizing fish urine and respiration). The model was implemented in Python 3.7 (Python Software Foundation, 2020).

Relevant model parameters were identified with an all-at-a-time (AAT), variancebased sensitivity analysis. The model was subsequently calibrated against literature data for fish growth, digestive content, and ammonia excretion. Calibration was performed using a set-membership approach, which is suitable to identify a set of feasible parameter vectors from limited data under measurement uncertainties, common in complex biological systems like aquaculture. Finally, nutrient dynamics and their uncertainties were evaluated using Monte Carlo simulations, sampling from the identified feasible parameter set.

\subsubsection{Mathematical model}

In what follows, the overall mass balance and the nutrient mass balances are introduced.

\section{Overall mass balance}

The overall mass balance in fish is described by a system of three differential equations with corresponding state variables for dry mass of fish $\left(m_{f s h}\right)$, contents of the digestive system $\left(m_{d i g}\right)$, and contents of the urinary system $\left(m_{\text {uri }}\right)$ (see Fig. 5.1):

$$
\begin{aligned}
& \frac{d m_{f s h}}{d t}=\phi_{u p t} \\
& \frac{d m_{d i g}}{d t}=\phi_{f e d}-\phi_{d i g-o u t} \\
& \frac{d m_{u r i}}{d t}=\phi_{d i g-u r i}-\phi_{\text {sol }}
\end{aligned}
$$

where $\phi_{u p t}$ is the mass flow rate uptake by fish for growth, $\phi_{f e d}$ is the mass flow rate of ingested feed, $\phi_{\text {dig-out }}$ is the total mass flow rate leaving the digestive system $\left(\phi_{\text {dig-out }}=\phi_{\text {dig-uri }}+\phi_{\text {upt }}+\phi_{\text {prt }}\right), \phi_{\text {dig-uri }}$ is the mass flow rate from the digestive to the urinary system, and $\phi_{\text {sol }}$ is the mass flow rate of soluble excretion.

Based on the work by Wik et al. (2009), excretion of faeces was modelled as particulate matter leaving the digestive system $\left(\phi_{p r t}\right)$. Similarly, excretion of urine and metabolism products through the gills was modelled as soluble matter leaving the urinary system $\left(\phi_{\text {sol }}\right)$. 


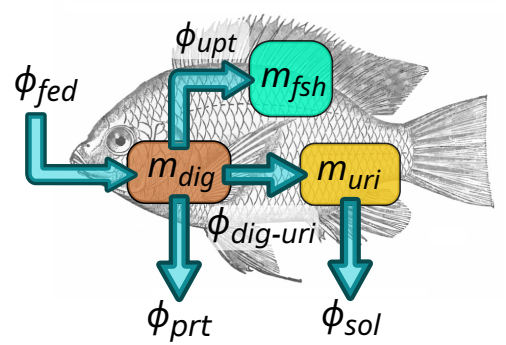

FIGURE 5.1: Schematic representation of the mass balances in fish.

The mathematical model uses three state variables for dry mass: fish $\left(m_{f s h}\right)$, digestive system content $\left(m_{d i g}\right)$, and urinary system content $\left(m_{u r i}\right)$. The main flows are: feed $\left(\phi_{f e d}\right)$, uptake to body mass $\left(\phi_{u p t}\right)$, excretion of particulate matter (faeces) $\left(\phi_{p r t}\right)$, transfer from digestive system to urinary system $\left(\phi_{d i g}-u r i\right)$, and excretion of soluble matter (respiration and urine) $\left(\phi_{\text {sol }}\right)$. Tilapia illustration:

Chromis niloticus by W H Flower, public domain (pd-old).

Emptying flow rates from the digestive and urinary systems $\left(\phi_{\text {dig-out }}\right.$ and $\left.\phi_{\text {sol }}\right)$ were modelled as first order processes with time constants $\tau_{\text {dig }}$ and $\tau_{u r i}$, respectively:

$$
\begin{aligned}
\phi_{\text {dig-out }} & =1 / \tau_{\text {dig }} * m_{\text {dig }} \\
\phi_{\text {sol }} & =1 / \tau_{\text {uri }} * m_{\text {uri }}
\end{aligned}
$$

Mass flow rates $\phi_{u p t}$ and $\phi_{p r t}$ were calculated as fractions of $\phi_{\text {dig-out }}$ :

$$
\begin{aligned}
& \phi_{u p t}=k_{\text {upt }} * \phi_{\text {dig-out }} \\
& \phi_{\text {prt }}=k_{\text {prt }} * \phi_{\text {dig-out }}
\end{aligned}
$$

where the mass flow fractions $k_{u p t}$ and $k_{p r t}$ were assumed constant throughout growth.

Finally, the mass flow rate from digestive to urinary system was calculated by completing the mass balance:

$$
\phi_{\text {dig-uri }}=\phi_{\text {dig-out }}-\phi_{\text {upt }}-\phi_{\text {prt }}
$$

In summary, the overall mass balance with state variables $m_{f s h}, m_{d i g}$, and $m_{u r i}$ has four unknown parameters (to be calibrated): $\tau_{d i g}, \tau_{u r i}, k_{u p t}, k_{\text {prt }}$. 


\section{Nutrient balances}

The nutrients modelled were $C, N, P, K, C a, M g$, and $N a$. Each of these nutrients $(j)$ was modelled following the dynamics of the overall mass balances:

$$
\begin{aligned}
& \phi_{j-u p t}=k_{j, u p t} x_{j, f e d} \phi_{\text {dig-out }} \\
& \phi_{j-f c s}=k_{j, p r t} x_{j, f e d} \phi_{\text {dig-out }}
\end{aligned}
$$

where $\phi_{j, u p t}$ is the mass flow rate taken up by fish during growth, and $\phi_{j, f c s}$ is the mass flow rate in faeces. Constants $k_{j, u p t}$ and $k_{j, p r t}$ are the proportions of each nutrient fed that ends in fish mass and particulate matter excretion, respectively. These constants were determined directly from measurements presented in literature (see Table A5.2 in the Appendix of this chapter). $x_{j, f e d}$ is the known fraction of nutrient $j$ in feed.

Mass flow rates in soluble matter excretions were calculated based on the volumetric flow rate through the urinary system $\left(\phi_{v}\right.$,dig-uri $)$, assuming constant density $(\rho)$, and constant nutrient concentration $C_{j, u r i}=\phi_{j, d i g-u r i} / \phi_{v, d i g-u r i}$ :

$$
\phi_{j, s o l}=C_{j, u r i} \phi_{v, s o l}=\frac{\phi_{j, d i g-u r i}}{\phi_{\text {dig-uri }} / \rho} \frac{\phi_{\text {sol }}}{\rho}
$$

Therefore:

$$
\phi_{j, s o l}=k_{j, s o l} x_{j, f e d} \frac{\phi_{\text {dig-out }}}{\phi_{\text {dig-uri }}} \phi_{\text {sol }}
$$

where constant $k_{j, s o l}$ is the proportion of nutrient fed that ends in soluble excretions.

\section{Model outputs}

Additionally, model outputs were introduced to relate the state variables to the measured variables: fish fresh mass $\left(m_{f s h, f r}\right)$, normalized mass in digestive system $\left(m_{d i g, n}\right)$, and normalized flow rate of total ammonia nitrogen $(T A N)$ excretion $\left(\phi_{T A N, n}\right)$. These relationships are given by:

$$
\begin{aligned}
& m_{f s h, f r}=m_{f s h} / k_{D M R} \\
& m_{d i g, n}=m_{d i g} / m_{f e d, d a y} \\
& \phi_{T A N, n}=k_{T A N, s o l} \cdot \phi_{N, s o l} / m_{f e d, d a y}
\end{aligned}
$$

where the state variables $m_{f s h}$ and $m_{\text {dig }}$ indicate dry mass $[g D M], k_{D M R}$ is the dry mass ratio (assumed constant throughout growth), $k_{T A N, s o l}$ is the fraction of soluble $N$ excretion that corresponds to $T A N$, and $\phi_{N, \text { sol }}$ is the excretion flow rate of soluble 
$N$ following from Eqn. 5.11. The digestive mass and TAN excretion are normalized against the daily mass of feed $\left(m_{f e d, d a y}\right)$.

\section{Feeder}

Fish feeding is a control input that was assumed to follow feed-forward control (i.e. without updated information about the actual fish mass beyond the initial value). This automatic feeder was modelled based on a prediction of fish fresh mass from a simple quadratic equation $\left(m_{f s h, q}\right)$, calibrated against data from Santos et al. (2008), Allaman et al. (2013), and Gullian-Klanian and Arámburu-Adame (2013):

$$
m_{f s h, q}=m_{0, f s h, f r}+0.239 \cdot t_{q}+0.0075 \cdot t_{q}^{2}
$$

where $m_{0, f s h, f r}$ is the initial fresh mass of fish, and $t_{q}$ in units of $[d]$. Such simple empirical models are commonly used to predict fish growth, but they do not describe the full range of growth (Belal, 2005). This empirical quadratic model was used in the present study to better represent real and uncertain growth conditions, where the growth predicted by the feeder may not match the actual growth (represented by the mass balance model).

Feed rate for each feeding was calculated assuming that feed is consumed within one simulation time step $\Delta t$ :

$$
\phi_{f e d}=\frac{k_{D F R} \cdot m_{f s h_{q}}}{\Delta_{t} \cdot n_{f e d}}
$$

where $k_{D F R}$ is the daily feed ratio ( $k g$ feed per $k g$ fish), and $n_{f e d}$ is the number of feeds per day.

During the first day of the simulation, fish feeding was assumed to take place once per day, to match the experimental conditions of the measured variables $m_{d i g, n}$ and $\phi_{T A N, n}$. Subsequently, all feeding was assumed to take place three times per day (at 8:00, 12:00 and 16:00 hr), to match common commercial and experimental conditions for growth. 


\section{Model assumptions}

The main assumptions for the structure and implementation of this model are:

- The model describes single fish growth, but it can be applied to multiple fish in a tank assuming that they are all in the same growth stage. This study includes model calibration for the growout stage, starting from a fish fresh mass of ca. $100 \mathrm{~g}$.

- The mass balances describe net effects, i.e. the end result of all short-term physiological nutrient exchanges between fish, feed and water.

- All model parameters are constant throughout the specified growth stage.

- Every nutrient follows the dynamics of the overall mass balance. Only the proportions splitting uptake, particulate excretions and soluble excretions are different between nutrients.

- There is no delay in excretions (based on the analysis by Wik et al. (2009)), and a single time constant for emptying of digestive and urinary systems was used.

- Reference model values were assumed as presented in the Appendix of this chapter .

\subsubsection{Sensitivity analysis method}

Relevant model parameters were identified using an all-at-a-time (AAT), variancebased sensitivity analysis (Sobol, 2001; Saltelli, 2002; Saltelli et al., 2010). This method is implemented in the package SALib (Herman et al., 2019), and computes first-order (S1), second-order (S2), and total (ST) sensitivity indexes. S1 indexes reflect the effect of variations of each single parameter on the variance of each model output. S2 indexes reflect the effect of interactions between pairs of parameters on the variance of each model output. ST indexes reflect the effect of each parameter on the variance of each model output, given variations in all parameters.

All four model parameters in the overall mass balances $\left(\tau_{d i g}, \tau_{u s i}, k_{p r t}, k_{u p t}\right)$ were included in the analysis. Furthermore, initial conditions $m_{0, f s h}$ and $m_{0, u r i}$ were also included. $m_{0, f s h}$ was included to account for the large variations commonly present in commercial fish production. $m_{0, \text { uri }}$ was included to account for the standard metabolic rates, i.e. the minimum level of energy consumption related to respiration, associated with $\mathrm{CO}_{2}$ and TAN excretions, and modelled as a constraint on the minimum mass present in the urinary system. $m_{0, \text { dig }}$ was not included in the analysis because it is a fixed value, calculated directly from the feed provided, assuming that all feed was eaten. 
The sensitivity indexes were computed for the measured variables available in literature (and matching model outputs) $m_{f s h, f r}, m_{d i g, N}$, and $\phi_{T A N, N}$. The indexes were computed for different instants, to identify possible changes through time due to the presence of multiple model outputs with different time scales. The instants were chosen at regular intervals, but different between model outputs, to match the time scales of the literature data. Detailed settings for the sensitivity analysis implementation are provided in the Appendix of this chapter.

\subsubsection{Literature data}

All data for model calibration was obtained from literature. Available data determined the choice for model outputs: fish growth, digestive system content, and TAN excretions. Only data series that match or approximate common commercial growth conditions were selected.

Regarding digestive mass $\left(m_{d i g, n}\right)$ and TAN excretions $\left(\phi_{T A N, n}\right)$, differences in experimental feeding regimes and schedules were standardized by expressing all literature measurements per mass of (single) feed provided at the start of the experiments.

Data for digestive mass $\left(m_{d i g, n}\right)$ was obtained from Richter et al. (2003), Riche et al. (2004), and Gómez-Peñaranda and Clavijo-Restrepo (2012). These studies measured the dry mass stomach content in tilapia after feeding, or an equivalent indicator, through a period of up to $12 \mathrm{hr}$. Fish fresh mass was between $100-300 \mathrm{~g}$. Based on the zero-delay observed in faeces excretion by tilapia, it was assumed that the stomach content is representative of the entire digestive system, neglecting the effect of any additional residence time in the intestines.

Data for TAN excretions $\left(\phi_{T A N, n}\right)$ was obtained from Obirikorang et al. (2015) and Obirikorang et al. (2017). These studies determined the excretion rate of ammonia after feeding through a period of up to $24 \mathrm{hr}$. Fish fresh mass was between $50-65 g$.

Data for fish growth $\left(m_{f s h, f r}\right)$ was obtained from Allaman et al. (2013) and Santos et al. (2008). Measurements for fish fresh mass were selected around $100 \mathrm{~g}$, which is representative for both available datasets of digestive mass $(100-300 \mathrm{~g})$ and TAN excretion $(50-65 g)$.

For the set-membership calibration, data bounds for digestive mass $\left(m_{\text {dig,n }}\right)$ and TAN excretion $\left(\phi_{T A N, n}\right)$ were generated for measurement time steps $\Delta t_{k} \geq 2 \mathrm{hr}$, locating the minimum and maximum values at intervals $t_{k} \pm 1 \mathrm{hr}$. In this way, multiple data sources can be compiled into single data bounds, which account for possible errors due to handling and weighting time of the fish stomach contents, and diffusion times of TAN in the fish tanks (independent variable error). Due to data 
availability, measurement instants start at $t=0 \mathrm{hr}$ for $m_{d i g, n}$, and at $t=1 \mathrm{hr}$ for $\phi_{T A N, n}$.

For further details regarding data processing, the reader is referred to the data files in the model documentation (Reyes Lastiri, 2020), and added as supplementary materials.

\subsubsection{Set-membership algorithm}

Multiple data sets of fish growth and excretions are available in literature. However, each study was conducted under different experimental conditions, which are not always reported in detail. These unknown variations introduce so-called sampling errors to the modelling process, which cannot be directly quantified. In addition, data from similar experimental conditions is limited and contains measurement uncertainties.

A preliminary study of the model presented in this paper, with least-squares calibration and linear uncertainty propagation, showed that the estimated parameter uncertainties were not sufficient to reproduce the large variations in the measured data. Possible causes for this limitation are the sequential calibration in separate steps for growth and excretions, as well as the assumptions for linear uncertainty propagation. This limitation suggests the use of an alternative approach, using all time scales simultaneously.

Model calibration was performed with a set-membership approach, which is suitable for limited data under measurement uncertainties (Milanese et al., 1996; Keesman, 2011; Cerone et al., 2014). In this paper, sampling, measurement and modelling errors were described in terms of bounded output errors. Hence, the data is assumed to be point-wise bounded for each measurement instant $(k)$ in the interval $\left[z_{k, \text { min }}, z_{k, \text { max }}\right]$. The calibration objective is then to identify a feasible parameter set (FPS) that produces model predictions within the data bounds. Formally, the FPS is defined as:

$$
F P S:=\left\{\vartheta \in \mathbb{R}^{p} \mid\left(z_{k, \text { min }} \leq y_{k}(\vartheta) \leq z_{k, \text { max }}\right) \text { for } k=1,2, \ldots\right\}
$$

The set-membership calibration used the so-called vorsetmembership algorithm (Reyes Lastiri, 2020). This algorithm approximates the feasible parameter set (FPS), and characterises it by means of a collection of (hyper)spheres. These spheres are iteratively generated from a Voronoi tessellation of unfeasible parameter vectors found by random sampling. 
The algorithm also quantifies deviations of model outputs from the data bounds, defined as underestimation $(U E)$ and overestimation $(O E)$, per model output $(y)$, based on errors $\varepsilon_{O E}$ and $\varepsilon_{U E}$, and the width of individual output intervals:

$$
\begin{gathered}
\varepsilon_{U E}:=\sum_{k} \max \left[\left(y_{k}\left(\vartheta_{F}\right)-z_{k, \text { min }}\right) \geq 0\right]+\sum_{k} \max \left[\left(z_{k, \text { max }}-y_{k}\left(\vartheta_{F}\right)\right) \geq 0\right] \\
\varepsilon_{O E}:=\sum_{k} \max \left[\left(z_{k, \text { min }}-y_{k}\left(\vartheta_{U}\right)\right) \geq 0\right]+\sum_{k} \max \left[\left(y_{k}\left(\vartheta_{U}\right)-z_{k, \text { max }}\right) \geq 0\right] \\
U E:=\varepsilon_{U E, y} / \sum_{k}\left(z_{k, \text { max }}-z_{k, \text { min }}\right) \\
O E:=\varepsilon_{O E, y} / \sum_{k}\left(z_{k, \text { max }}-z_{k, \text { min }}\right)
\end{gathered}
$$

where $\vartheta_{F}$ and $\vartheta_{U}$ are feasible and unfeasible parameter samples obtained from the approximate FPS. See Chapter 4 for details on the method.

The metrics $U E$ and $O E$ were used to quantify the extent of the data variability reflected by the model prediction uncertainty. An analysis of the magnitude and instants of these deviations in each model output was used to identify possible improvements for future experimental design.

The parameters in $\mathbb{R}^{p}$ and the resulting FPS were normalized to the same bounds as used for the sensitivity analysis. Parameter constraints were applied to improve identifiability (See the Appendix of this chapter).

\subsubsection{Uncertainty propagation}

The set-membership approach in this paper assumes that the uncertainty in the parameters, described by the approximate FPS, encompasses all uncertainties in the modelling process (e.g. input, measurement, model structure, sampling, and independent variable errors).

Uncertainty propagation in the model prediction for all nutrient excretions $\left(\phi_{p r t, j}\right.$ and $\left.\phi_{s o l, j}\right)$ was assessed with Monte Carlo simulations through sampling of the FPS. The model prediction uncertainty was quantified in terms of a coefficient of variation $(\mathrm{CV})$, defined in this paper with respect to the model output median $\tilde{y}$ as:

$$
C V:=\left(y_{\max }-y_{\min }\right) / \tilde{y}
$$

Nutrients with the largest CV were identified to suggest objectives for future experimental design, focusing attention on the sampling times and nutrients that would better improve knowledge on the dynamics of nutrient excretions. 


\subsection{Results and discussion}

An initial model evaluation was performed to assess the model behaviour against the measured data bounds (Fig. 5.2), and to help identify an initial set of parameter values. Detailed settings for the reference model are provided in the Appendix of this chapter.

The model output for fresh fish mass $\left(m_{f s h, f r}\right)$ follows the expected non-linear growth of fish, and the dynamics of the feed provided $\left(\phi_{f e d}\right)$. Therefore, this semiphysical modelling approach (Eqns. 5.1 to 5.14) is able to reproduce the growth dynamics with a time scale of several months, based on the mass balance dynamics of feed uptake and excretions with a time scale of hours.
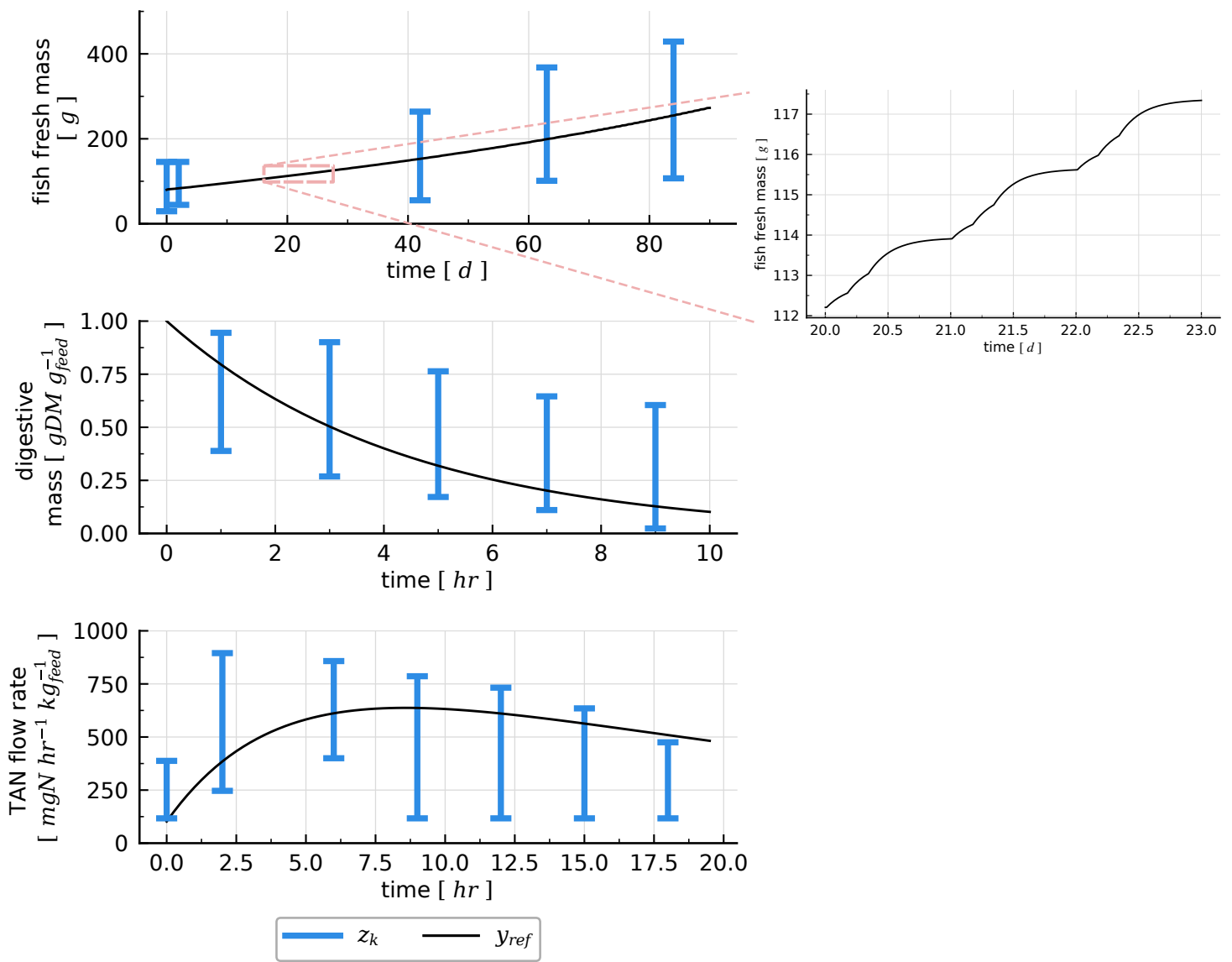

FIGURE 5.2: Data bounds at measurement instants $\left(z_{k}\right)$, and reference model outputs $\left(y_{\text {ref }}\right)$. Notice that the fish growth model output follows both the non-linear dynamics (top left) and the fish feed schedule with 3 feeding times per day (close-up). Notice also that $y_{\text {ref }}$ for TAN excretion flow rate does not yet fit within all data bounds. In the subsequent set-membership calibration step, the objective is to identify a feasible parameter set which generates model outputs fitting within all data bounds. 
An analysis of the model structure as a linear time independent (LTI) system is presented in the Appendix of this chapter. The eigenvalues of the model, without a maintenance term, show that the model is critically stable, where fish growth follows the quadratic feeding strategy.

\subsubsection{Sensitivity analysis}

An all-at-a-time (AAT) sensitivity analysis was performed to identify the most relevant parameters of the model, out of the six that were initially chosen (four model parameters and two initial conditions). The resulting Sobol sensitivity indexes S1 and ST are shown in Fig. 5.3. Notice that the sensitivity indexes change with time. In particular, the sensitivities of $m_{f s h, f r}$ and $m_{d i g, n}$ to $m_{0, f s h}$ decrease with time, whereas $k_{\text {upt }}$ and $\tau_{\text {dig }}$ become increasingly relevant. In contrast, the sensitivity of $\phi_{T A N, n}$ to $m_{0, f s h}$ increases with time. This result demonstrates that it is necessary to develop a model accounting for the different time scales of the model outputs.

A general guideline for the selection of relevant parameters consists in prioritizing those with the highest S1 indexes, and discarding those with the lowest ST indexes, by fixing their values (Saltelli et al., 2008; Jaxa-Rozen and Kwakkel, 2018). Based on this guideline:

- Time constants $\tau_{d i g}, \tau_{u r i}$, the initial condition $m_{0, f s h}$, and the ratio $k_{u p t}$, returned the largest S1 indexes. Therefore, they were subsequently included in the calibration step. Although small, the effect of initial condition $m_{0, u r i}$ is also visible in the S1 index. Therefore, it was also included in the calibration step.

- The ratio $k_{\text {prt }}$ returned the lowest ST index. Therefore, it was subsequently excluded from the calibration step.

Large correlated effects were not observed in the S2 indexes. Some correlated effects were observed for time constant $\tau_{\text {uri }}$ and initial condition $m_{0, \text { uri }}$, with all other parameters on $\phi_{T A N, n}$, particularly at the start of the period. Their S2 indexes decrease in time from ca. $10^{-1}$ to $10^{-2}$. All other S2 indexes were in the order of magnitude of $10^{-3}$.

The aforementioned effects may suggest to exclude $m_{0, u r i}$ from the calibration step. However, $m_{0, u r i}$ is only measured indirectly on model output $f_{T A N, n}$, and it is affected by multiple physiological processes summarized as the net availability of all soluble nutrients. Such a variable is difficult to measure experimentally, and no values were found in literature. Therefore, it was decided to include $m_{0, u r i}$ in the calibration step, starting from a manually-adjusted reference value. 

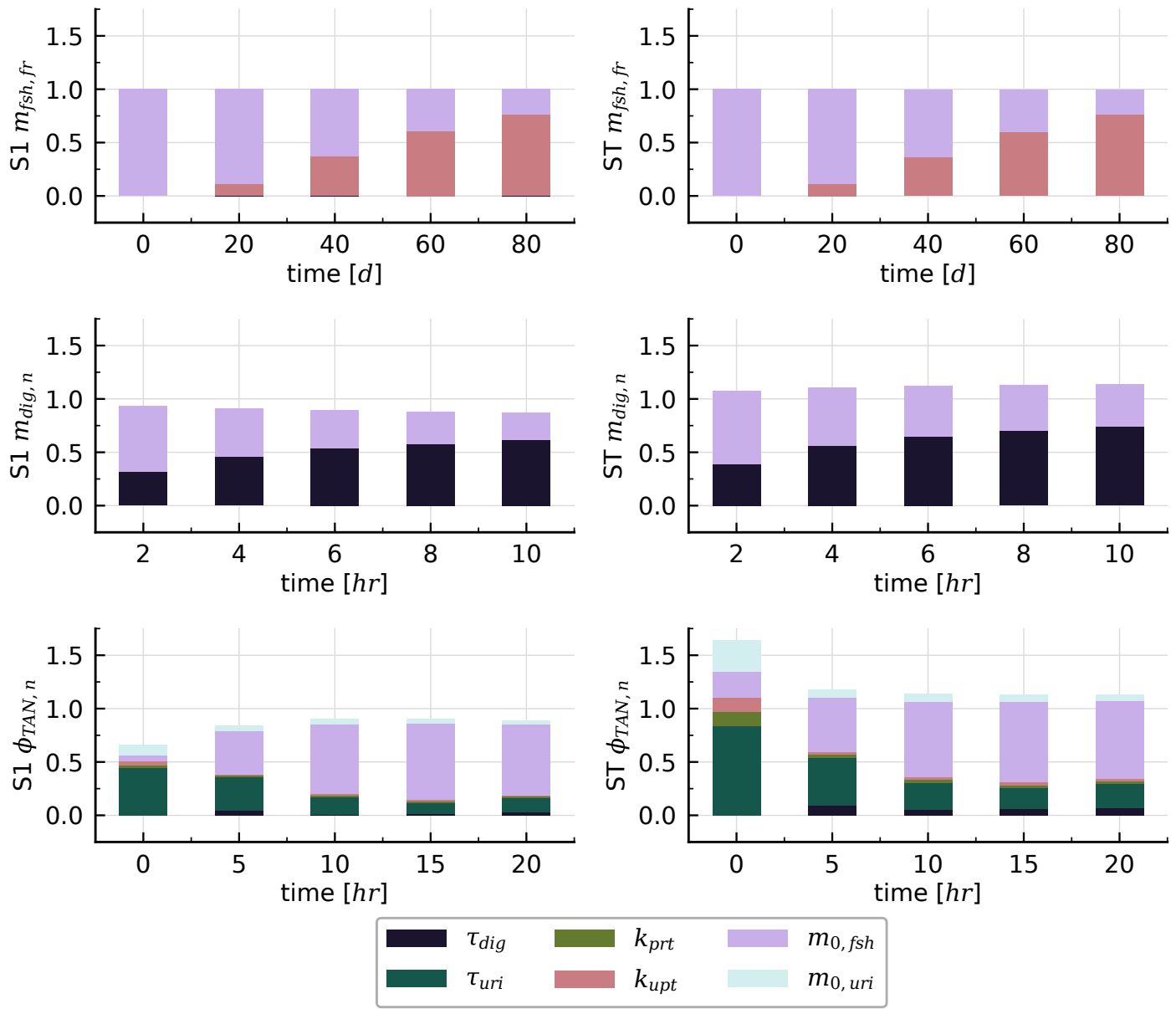

FIGURE 5.3: First-order (S1) and total (ST) indexes from the Sobol sensitivity analysis on each model output: fish fresh mass $\left(m_{f s h, f r}\right)$, digestive mass normalized to daily feed $\left(m_{d i g, n}\right)$ and TAN excretion normalized to daily feed $\left(\phi_{T A N, n}\right)$. Notice the different time scales for each of the model outputs.

\subsubsection{Set-membership calibration}

Set-membership calibration for model parameters $\tau_{d i g}, \tau_{u r i}, k_{u p t}, m_{0, f s h}$, and $m_{0, u r i}$, was initialized with the same parameter bounds of the sensitivity analysis. These parameter bounds were also used to normalize the parameter domain. Detailed settings for the calibration routine are provided in the Appendix of this chapter.

The resulting approximate FPS is shown in Fig. 5.4. The calibration was constrained to physically sensible parameter values (e.g. positive time constants). The effect of these constraints is observed in the clear cuts on the approximate FPS, which prevent it from extending indefinitely due to correlations between parameter estimates. These correlations were particularly accentuated in the (initially nonconstrained) parameter space projections $\left(\tau_{\text {dig }}, m_{0, u r i}\right)$ and $\left(\tau_{\text {uri }}, m_{0, u r i}\right)$, matching the expected effects from the S2 Sobol indexes observed in the sensitivity analysis. 


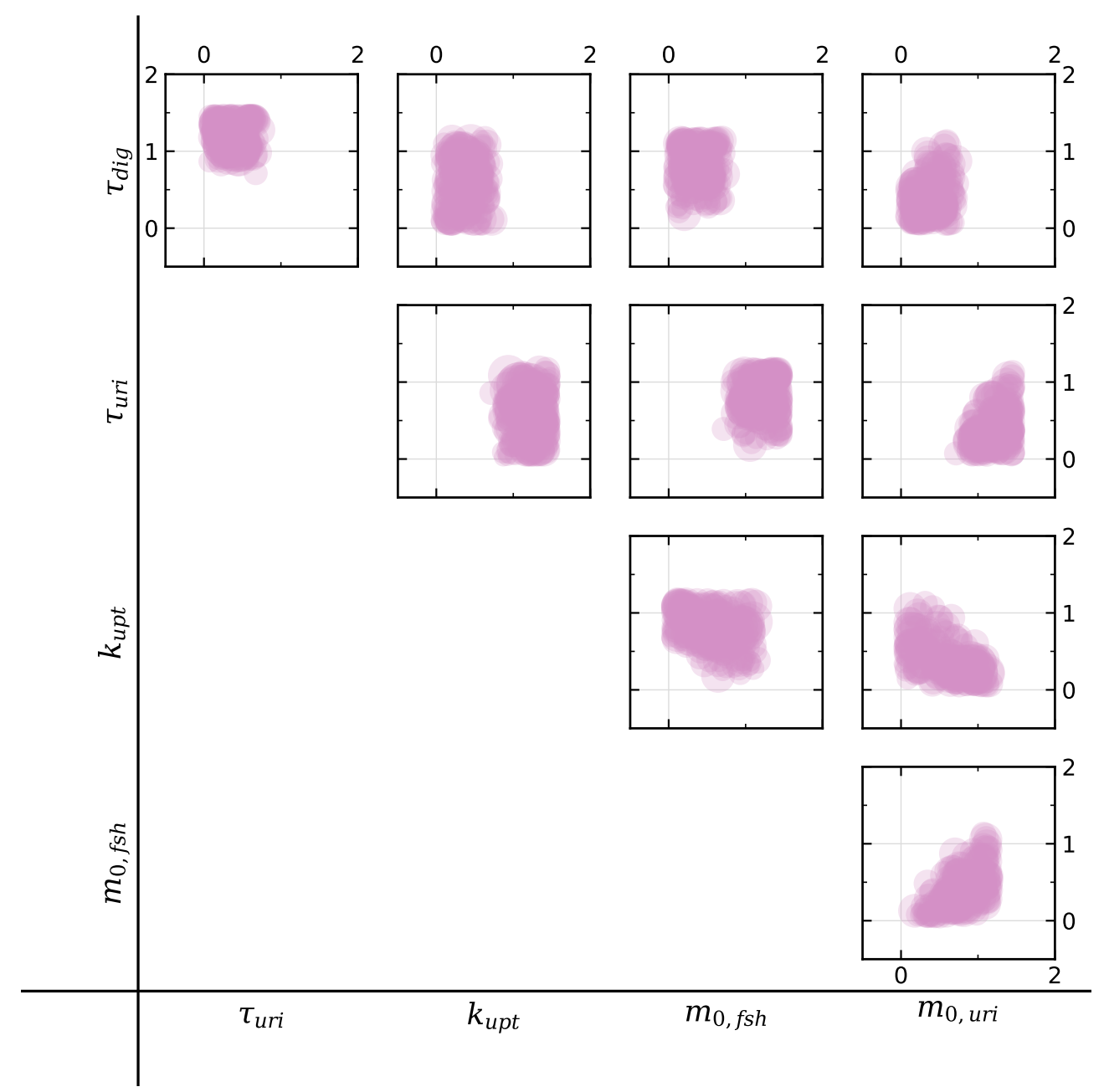

FIGURE 5.4: Approximate feasible parameter set (FPS) characterised by hypershperes. $2 \mathrm{D}$ projections of the $5 \mathrm{D}$ parameter space $\left(\mathbb{R}^{p}\right.$ with $\left.p=5\right)$. Parameter space normalized to the bounds of the sensitivity analysis, provided in the Appendix of this chapter.

However, the model showed a high underestimation (UE) for the uncertainty of $\phi_{T A N, N}$ during the corresponding time frame of the experiment (Fig. 5.5). The observed UE reflects that, in order to reproduce the low TAN excretions measured $10 \mathrm{hr}$ after feeding, the model predicts TAN excretion rates larger than the measured maximum in the first hours after feeding, resulting in $O E$, which the algorithm is designed to prevent. Based on this behaviour, we hypothesised two causes for the mismatch between model prediction and experimental measurements, related to the experimental conditions reported in the corresponding literature (Obirikorang et al., 2015; Obirikorang et al., 2017), namely: 

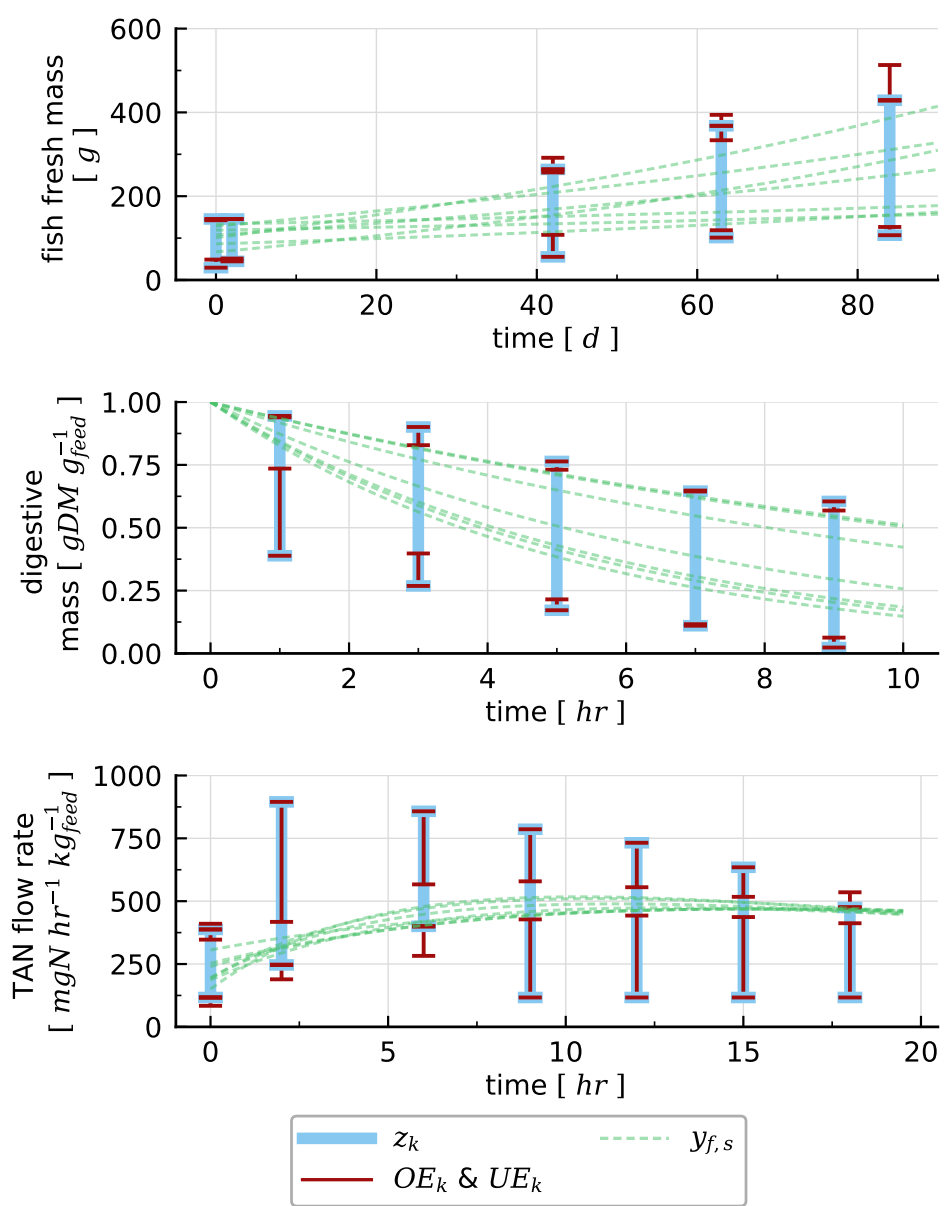

FIGURE 5.5: Evaluation of the approximate feasible parameter set (FPS) on the feasible model output set (FMOS), in terms of over and underestimation at measurement instants $\left(O E_{k}\right.$ and $\left.U E_{k}\right)$. A sampled subset of the feasible model outputs $\left(y_{f, s}\right)$ is included to illustrate the model prediction uncertainty. Notice the large $U E$ in the TAN excretion rate $\left(\phi_{T A N, n}\right)$ which shows that the parameter uncertainty cannot reproduce all data variability for this variable.

1. Starvation. Prior to the reported experiments, tilapia were starved for 24 and $48 \mathrm{hr}$, and, in one experiment, feed provided was half of the feed from previous days. The change in feed availability could lead to a higher $N$ uptake ratio in the first day of the experiment, as a physiological mechanism that compensates for the prior deficiency. This phenomenon is reflected by the calibrated model as a slow period of increase in TAN excretion during 6-7 days following the post-starvation day of the experiments, until stable levels are reached (Fig. 5.6). However, due to the assumption of constant nutrient uptake ratios, modelled uptake and excretion rates are not stabilized immediately after the first feeding following starvation, limiting model coverage of data variability, as observed in Fig. 5.5. 


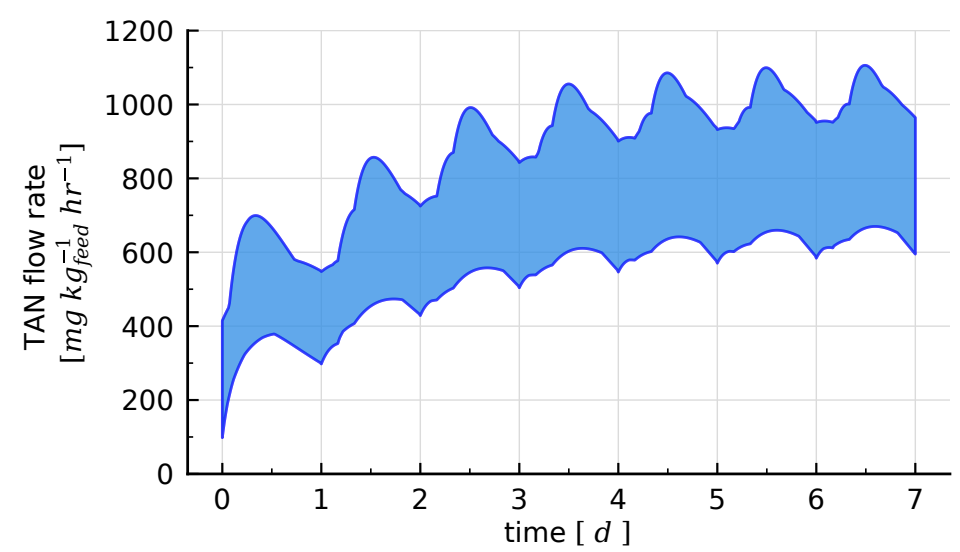

FIGURE 5.6: Bounded model prediction uncertainty for ammonia excretion rate $\left(\phi_{T A N, n}\right)$ following from the first day after starvation in experimental conditions. Notice that $\phi_{T A N, n}$ is low in the first day, and increases through one week, before reaching a stable level. Therefore, predicted $\phi_{T A N, n}$ levels are affected by the initial starvation conditions from the experimental measurements.

2. Closed tanks. Fish tanks were intermittently opened and closed to water circulation. The tanks remained closed during the period when water samples were collected, in order to calculate TAN excretion rates based on TAN accumulation. However, some teleosts are known to detoxify ammonia by converting it to urea (ureogenesis), a process which is energetically expensive and thus avoided under regular conditions. This phenomenon has been confirmed in one species of tilapia, Alcolapia grahami (Randall, 2011). If ureogenesis is present in Oreochromis niloticus, closed tanks and subsequent ammonia accumulation could be the cause for very low TAN excretions after the peak, constraining the model predictions and resulting in high $U E$.

Starvation and closed tanks are part of experimental designs aimed at achieving controlled conditions to measure fish excretions. However, due to the complex interactions and feedback loops between fish and water, attempts to control the experimental conditions affect the existing stability in those interactions, effectively acting as disturbances on the system. Therefore, future experimental design aimed at improving the knowledge on nutrient dynamics could benefit from measurements under normal growth conditions with standard feeding and circulating water. 
A alternative interpretation of the mismatch between model and data, is the presence of structural modelling errors (i.e. the model is unable to reproduce TAN excretions under unusual or extreme conditions). In this case, future research could be oriented to identify and understand these extreme conditions. One possible way to improve model robustness would consist of adding a term for ammonia excretion inhibition. Such inhibition could be described in terms of a sigmoid function, reflecting the variation of ammonia forms linked to $\mathrm{pH}$ variations.

In summary, based on the set-membership calibration results:

1. Parameter uncertainty characterised by the approximate FPS results in model predictions with improved coverage of data variability, compared to leastsquares calibration with posterior uncertainty analysis based on linear error propagation. Based on the quantified underestimation $(U E)$, the model reproduces $69 \%$ of the data bounds for fish growth $\left(m_{f s h, f r}\right)$, and $75 \%$ of the data bounds for mass in the digestive system $\left(m_{d i g, n}\right)$, However, the model underestimates uncertainties in $\phi_{T A N, n}$, covering only $28 \%$ of the data bounds.

2. Future experimental research aimed at understanding excretion dynamics should account for possible inhibition of TAN excretions. The observations in this paper lead to suggest the use of normal growth conditions, such as regular feed and circulating water.

\subsubsection{Uncertainty propagation on nutrient excretions}

Uncertainty propagation was computed using Monte Carlo simulations, based on both the approximate FPS (Fig. 5.4) and uncertainty in literature-based nutrient ratios $k_{j, p r t}$ and $k_{j, u p t}$ (see Table A5.2 in the Appendix of this chapter). Model prediction uncertainty of nutrient excretions is shown in Fig. 5.7 for $\mathrm{N}, \mathrm{P}$, and $\mathrm{K}$. Notice that soluble excretions have a larger $\mathrm{CV}$ throughout the simulation period, whereas particulate excretions only have large CV immediately after feeding. Therefore, attention should focus on soluble excretions.

The complete list of maximum CV per soluble nutrient is provided in Table 5.1. The largest $\mathrm{CV}$ is observed in $\mathrm{P}, \mathrm{Ca}$ and $\mathrm{Na}$. In order to ensure normal growth conditions and prevent metabolic feedback loops, a possible experimental design could focus on measuring nutrients in the effluent of the fish tanks. These measurements would require the addition of a sub-model and accompanying model parameter, e.g. a time constant related to mixing in the tanks. In summary, focusing on the largest prediction uncertainties presents the best opportunity for improving the model accuracy. 

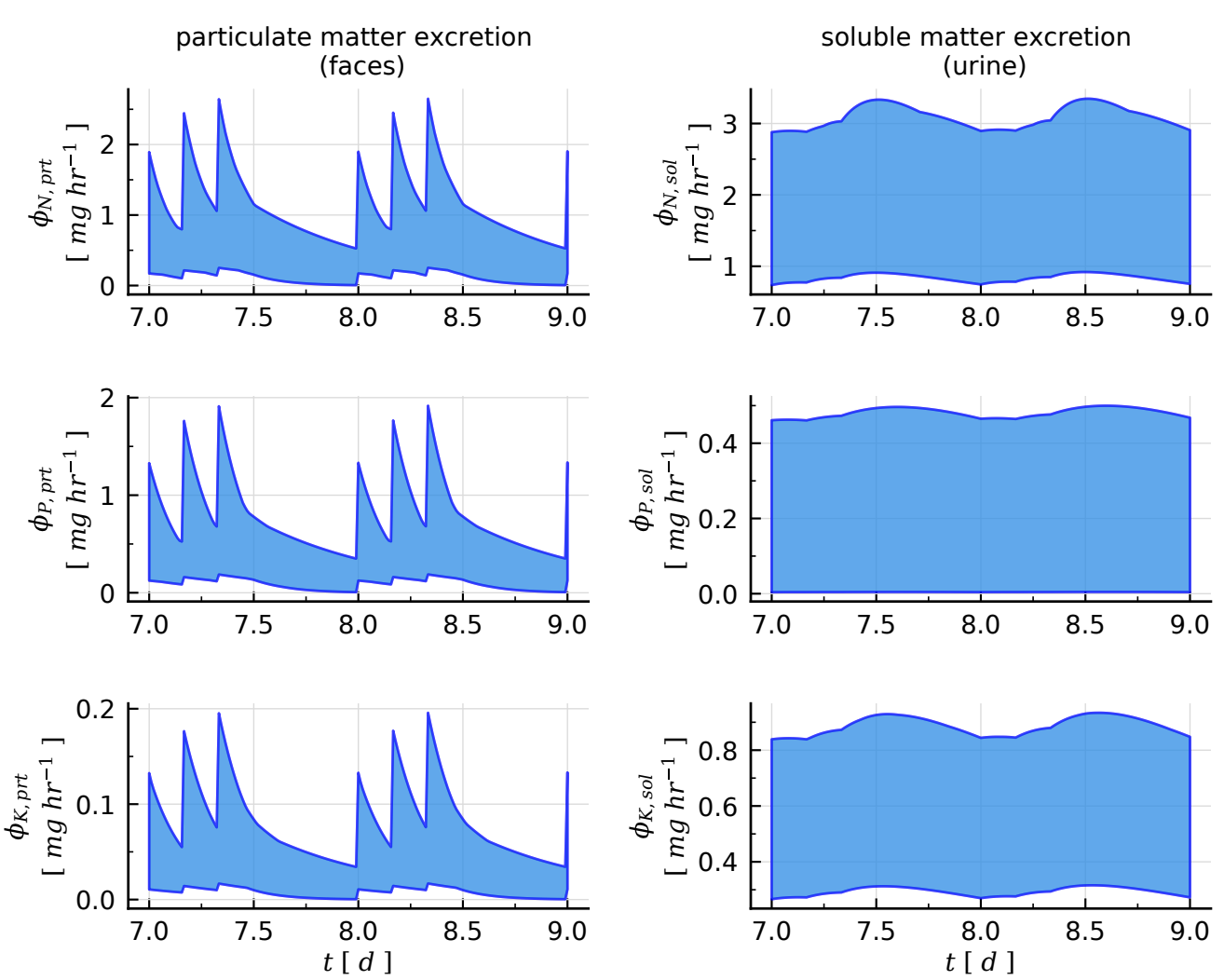

FIGURE 5.7: Model prediction uncertainty for total fish excretion rates of N, P and $\mathrm{K}$ in particulate form (left column) and soluble form (right column).

TABLE 5.1: Maximum coefficient of variation (CV) of soluble excretions.

\begin{tabular}{lc}
\hline Nutrient & $\begin{array}{c}\mathrm{CV} \phi_{j, \text { sol }} \\
{[\%]}\end{array}$ \\
\hline $\mathrm{C}$ & 50 \\
$\mathrm{~N}$ & 57 \\
$\mathrm{P}$ & 98 \\
$\mathrm{~K}$ & 50 \\
$\mathrm{Ca}$ & 67 \\
$\mathrm{Mg}$ & 55 \\
$\mathrm{Na}$ & 72 \\
\hline
\end{tabular}




\subsection{Conclusions}

A semi-physical model for nutrient dynamics was developed, using mass balances to describe uptake and excretions in particulate and soluble forms. The model contains only four parameters and describes dynamics at two time scales: hourly dynamics of excretions and monthly dynamics of growth. These time scales were used for an all-at-a-time sensitivity analysis, and set-membership calibration of the model.

The model prediction based on uncertainties of the four model parameters and two of the initial conditions, explained most of the data variability for fish growth $(84 \%)$ and digestive mass (75\%). However, the model only explained $28 \%$ of the data variability for ammonia excretions. One possible cause for this mismatch was the use of closed tanks and starvation in the experimental measurements, which may disturb the dynamics of ammonia excretion. Therefore, it is suggested to use common growth conditions when measuring ammonia excretion dynamics in fish tanks. Additionally, regarding research aimed at understanding extreme conditions (e.g. for risk assessment), it is suggested to improve the model quality by adding a term for inhibition of ammonia excretion.

Analysis of the uncertainty propagation on nutrient excretions revealed that $P$, $\mathrm{Ca}$ and $\mathrm{Na}$ in soluble excretions present the largest coefficient of variation. Therefore, to improve prediction accuracy, future experimental measurements should focus on these nutrients, using common growth conditions. 


\subsection{Appendix}

\section{LTI system}

The system of differential equations (Eqns. 5.1 - 5.3) can be expressed as a linear time independent (LTI) system, of the form:

$$
\frac{d x}{d t}=A x+B u
$$

with state variables:

$$
x=\left[\begin{array}{l}
m_{f s h} \\
m_{d i g} \\
m_{u r i}
\end{array}\right]
$$

and external input:

$$
u=\phi_{f e d}
$$

The system matrix is given by:

$$
A=\left[\begin{array}{ccc}
-k_{m n t} & k_{u p t} / \tau_{\text {dig }} & 0 \\
0 & -1 / \tau_{\text {dig }} & 0 \\
0 & \left(1-k_{u p t}-k_{\text {prt }}\right) / \tau_{\text {dig }} & -1 / \tau_{u r i}
\end{array}\right]
$$

and the input matrix is given by:

$$
B=\left[\begin{array}{l}
0 \\
1 \\
0
\end{array}\right]
$$

For this notation, a flow rate for maintenance $\left(\phi_{m n t}\right)$ leaving from $m_{f s h}$ is included but assumed to be close to zero $\left(k_{m n t} \approx 0\right)$ :

$$
\phi_{m n t}=k_{m n t} m_{f s h}
$$

The eigenvalues $(\lambda)$ of matrix $A$ are real and negative:

$$
\lambda=\left[\begin{array}{lll}
-1 / \tau_{\text {dig }} & -1 / \tau_{\text {uri }} & -k_{m n t}
\end{array}\right]
$$

which shows that the LTI system is asymptotically stable, and will not show any oscillatory behaviour. Assuming $k_{m n t}=0$, makes the LTI system critically stable. 


\section{Reference model settings}

Time step set to $\Delta t=15 \mathrm{~min}$, based on the hourly dynamics of excretions, and larger than the minimum time for fish feeding, as observed by Obirikorang et al. (2017) (i.e. neglecting dynamics during feeding).

TABLE A5.1: Reference model settings with initial parameter values.

\begin{tabular}{lll}
\hline Parameter & Value & Units \\
\hline$m_{0, f s h}$ & 80 & {$[g D M]$} \\
$m_{0, \text { dig }}$ & $1 \times 10^{-6}$ & {$[g D M]$} \\
$m_{0, \text { uri }}$ & $1 \times 10^{-6}$ & {$[g D M]$} \\
$\tau_{\text {dig }}$ & 4.5 & {$[h r]$} \\
$\tau_{\text {uri }}$ & 20.0 & {$[h r]$} \\
$k_{\text {upt }}$ & 0.3 & {$[-]$} \\
$k_{\text {prt }}$ & 0.2 & {$[-]$} \\
\hline
\end{tabular}

TABLE A5.2: Feed composition and intra-corporeal balances of nutrients

(j) as percentage of nutrient consumed [min, max].

\begin{tabular}{lccc}
\hline Nurient & $\begin{array}{c}\text { Feed } \\
x_{j, f e d}\end{array}$ & $\begin{array}{c}\text { Uptake } \\
k_{j, \text { upt }}\end{array}$ & $\begin{array}{c}\text { Particulate excretion } \\
k_{j, p r t}\end{array}$ \\
\hline $\mathrm{C}$ & 0.40 & {$[0.29,0.37]$} & {$[0.13,0.17]$} \\
$\mathrm{N}$ & 0.05 & {$[0.40,0.48]$} & {$[0.05,0.17]$} \\
$\mathrm{P}$ & 0.01 & {$[0.27,0.79]$} & {$[0.27,0.59]$} \\
$\mathrm{K}$ & 0.01 & {$[0.22,0.26]$} & {$[0.02,0.06]$} \\
$\mathrm{Ca}$ & 0.02 & {$[0.29,0.45]$} & {$[0.16,0.36]$} \\
$\mathrm{Mg}$ & 0.003 & {$[0.19,0.23]$} & {$[0.11,0.27]$} \\
$\mathrm{Na}$ & 0.005 & {$[0.40,0.60]$} & {$[0.05,0.21]$} \\
\hline
\end{tabular}

Data reported by Seawright et al. (1998), Schneider et al. (2004), and Montanhini Neto and Ostrensky (2015).

Soluble percentages calculated by completing the balance: $k_{j, s o l}=1-k_{j, u p t}-k_{j, p r t}$.

Initial mass $m_{0, f s h}=80 \mathrm{~g}$ was based on the first set of measurements available before $100 \mathrm{~g}$ which was chosen as a representative mass for the literature data sets for digestive $(100-300 \mathrm{~g})$ and urinary systems $(50-65 \mathrm{~g})$. Initial masses $m_{0, \text { dig }}$ and $m_{0, u r i}$ are small amounts to match the experimental conditions of starvation in the literature data.

Bounds for time constant $\tau_{\text {dig }}$ were based on the expected first order dynamics proposed in Wik et al. (2009) and the corresponding literature data in Richter et al. (2003), Riche et al. (2004), and Gómez-Peñaranda and Clavijo-Restrepo (2012). Similarly, bounds for time constant $\tau_{\text {uri }}$ were based on the expected (overall) second 
order dynamics proposed in Wik et al. (2009) and the corresponding literature data in Obirikorang et al. (2015) and Obirikorang et al. (2017).

Bounds for fraction $k_{u p t}$ were determined around a $k_{F C R}=1.2\left[k_{g_{f e e d}} k_{f_{f s h}, f r}^{-1}\right]$ (Timmons and Ebeling, 2010), and a $k_{D M R}=0.31$ (Schneider et al., 2004; Montanhini Neto and Ostrensky, 2015). Bounds for fraction $k_{f c s}$ were defined based on observations by Schneider et al. (2004).

Bounds for initial mass $m_{0, f s h}$ were based on the literature data in Allaman et al. (2013) and Santos et al. (2008). Bounds for initial mass $m_{0, u r i}$ were determined from manual adjustments on model output $\left.\phi_{T A N, n}\right|_{t=0}$ to approximate the corresponding data bounds, given the reference model settings.

Reference values for the nutrient balance coefficients $k_{j}$ were defined on as the median of the intervals in Table A5.2. For the Monte Carlo simulations, an uniform distribution in the intervals was assumed.

\section{Sensitivity analysis settings}

The Sobol sensitivity analysis was performed based on the same settings of the reference model evaluation (Appendix 5.5).

Parameter bounds (Table A5.3) were set around the reference values in Appendix 5.5 and based on ranges reported in the cited literature.

Sampling from the parameter bounds in Table A5.3 was performed using the Saltelli method, with $N=1200$, for a resulting sample size $n=N \cdot\left(2 \cdot n_{p}+2\right)=$ 16800.

The sensitivity indexes for each model output were computed for different time instants, based on the time scales from literature data.

$$
\begin{aligned}
t_{k, m-f s h} & =[0,20,40,60,80][d] \\
t_{k, m-d i g} & =[2,4,6,8,10][h r] \\
t_{k, f-T A N} & =[0,5,10,15,20][h r]
\end{aligned}
$$

The sensitivity indexes computed are defined as:

$$
\begin{aligned}
& S 1_{p i}=V_{p i}\left[E_{p \sim p i}(y \mid p i)\right] / V(y) \\
& S 2_{p i, p j}=E_{p \sim p i, p j}\left[V_{p i, p j}(y \mid p \sim p i, p j)\right] / V(y)-S 1_{p i}-S 1_{p j} \\
& S T_{p i}=E_{p \sim p i}\left[V_{p i}(y \mid p \sim i)\right] / V(y)
\end{aligned}
$$


Chapter 5. Set-membership uncertainty assessment of a mathematical model for nutrient balances in fish growth and excretions.

TABLE A5.3: Parameter bounds for the sensitivity analysis

\begin{tabular}{lll}
\hline Parameter & Bounds (min,max) & Units \\
\hline$\tau_{\text {dig }}$ & $(2.0,24.0)$ & {$[h r]$} \\
$\tau_{u r i}$ & $(6.0,32.0)$ & {$[h r]$} \\
$k_{\text {upt }}$ & $(0.05,0.45)$ & {$[-]$} \\
$k_{\text {prt }}$ & $(0.05,0.45)$ & {$[-]$} \\
$m_{0, f s h}$ & $\left(30 \cdot k_{D M R}, 120 \cdot k_{D M R}\right)$ & {$[g D M]$} \\
$m_{0, u r i}$ & $0.1,1.0$ & {$[g D M]$} \\
\hline
\end{tabular}

\section{Set-membership calibration settings}

The calibration algorithm was initialized with iterative Latin hypercube sampling of grid size $n_{\text {grd,ini }}=500$, until an initial vector of feasible parameters $\theta_{f, i n i}=50$ was obtained.

The estimation algorithm was run with default values, a grid size $n_{\text {grd }}=1000$, a sample size from the approximate FPS per iteration $n_{f p s}=250$, and a sample size from the boundaries of the approximate FPS per iteration $n_{b n d}=125$.

Model parameters $\tau_{\text {dig }}, \tau_{\text {uri }}, k_{\text {upt }}, m_{0, f s h}$, and $m_{0, \text { uri }}$, were constrained based on sensible physical limits ([min],[max]), as follows:

$$
\theta_{\text {cnstr }}=\left(\left[0.25,0.25,0.01,10 * k_{D M R}, 0.01\right],\left[48.0,48.0,0.6,150 * k_{D M R}, 2.0\right]\right)
$$




\section{Chapter 6}

\section{General discussion}

Aquaponics provides an opportunity for local, resilient food production, by combining growth of aquatic species and plants. However, this combination requires balancing the needs of multiple species.

This chapters reflects on the research questions addressed in the thesis, related to the experiences from system-level analysis and implementation of decoupled aquaponic systems, and to the experiences in modelling and simulation of aquaponics. Subsequently, it extends the reflection to the more general field of mathematical modelling of biological production systems, and the socio-economic challenges for the development of aquaponics and local food production.

\subsection{Research questions}

This section, reflects on the research questions presented in the General introduction, referring to the research chapters where they were addressed.

\section{Research question 1.}

What are the opportunities, challenges and possible solutions in closing cycles of water and nutrients in decoupled aquaponic systems?

Chapters 2 and 3 investigated system-level design and performance of decoupled aquaponic systems, regarding the use of water and macronutrients when connecting the loops of the recirculating aquaculture system (RAS) and the greenhouse recirculating hydroponic system (RHS). Chapter 5 investigated the dynamics and uncertainties of fish growth and excretions, aimed at reusing macronutrients from fish water. 


\section{Chapter 2}

The model-based study from Chapter 2 showed that it is possible to stabilize nitrogen concentration in the RAS loop by using a schedule based on fish feeding times when sending water from RAS to RHS, as opposed to a constant flow rate (Fig. 2.2). Further understanding of nutrient concentrations for reuse by plants requires models that describe nutrient dynamics and peaks in RAS, starting from fish excretions. However, there are large uncertainties in the predictions of nutrient concentrations in RAS. One possible approach to account for large uncertainties in experimental measurements and model predictions is the set-membership approach, which is covered in Capters 4 and 5 .

The model was also used to size a nutrient film technique (NFT) unit in the RHS, with the objective of minimising fresh water and nitrogen use, and wastewater discharge from RAS. The results showed that it is possible to find an optimum NFT size that meets these criteria (Fig. 2.3). However, an aquaponic system has multiple performance indicators, and an optimal design would require the balance of multiple trade-offs. Furthermore, these indicators have a high location specificity. For example, water availability in the Netherlands is not a constraint in greenhouse horticulture, and equipment is affordable and locally developed, but labour is relatively expensive; in contrast, water availability is very limited in Namibia, and labour is relatively inexpensive, but technological imports and technical training would be required (Nederhoff and Stanghellini, 2010; Goddek and Körner, 2019). Therefore, future research on the design of aquaponic systems should include a comprehensive quantitative analysis, based on indicators that reflect the trade-offs across RAS and RHS, and that can be used for either decoupled or coupled system configurations. The following key performance indicators are suggested:

- RHS productivity [ $k g \mathrm{~m}^{2} \mathrm{yr}^{-1}$ ]. Indicator proposed to quantify yearly plant production against production area. It can be quantified in terms of either greenhouse area (common indicator in greenhouse horticulture), or total area (RHS greenhouse + RAS building). Quantifying in terms of greenhouse area allows for comparison against common or local greenhouse production efficiencies. Quantifying in terms of total area provides a relation to the total initial investment, reflecting trade-offs when sizing RAS and RHS loops.

- RAS productivity $\left[\mathrm{kg} \mathrm{m}^{-3}\right.$ and $\left.\mathrm{kg} \mathrm{m}^{-2}\right]$. Indicator proposed to quantify yearly fish production against total fish tank volume (common indicator in aquaculture), and total production area. Quantifying in terms of fish tank volume allows for comparison against common or local RAS production efficiencies. Quantifying in terms of total production area, reflects trade-offs related to the initial investment when sizing RAS and RHS loops. 
- Water use efficiencies $\left[\mathrm{m}^{3} \mathrm{~kg}^{-1}\right]$. Indicator proposed to quantify total fresh water use against production of fish and plants. Water use efficiency is a common indicator in RAS and greenhouse horticulture production. This indicator reflects water savings accomplished by reusing water from fish for plants, or vice versa.

- Nutrient use efficiencies $\left[\mathrm{kg} \mathrm{kg}^{-1}\right]$. Indicator proposed to quantify the use of $N$ and $P$ in fertilizer against plant yield. This indicator reflects nutrient savings accomplished by reusing nutrients in the water from fish for plants. These nutrients are also related to environmental impacts like eutrophication.

- Nutrient discharge $\left[k g \mathrm{yr}^{-1}\right]$. Indicator proposed to quantify $N$ and $P$ discharge, macronutrients directly related to environmental impacts like eutrophication. In some places, discharge amounts are regulated. These nutrients are present in the sludge from RAS, and the water discharge from RAS and RHS.

- Payback period [yr]. Indicator proposed to quantify the economic performance of an aquaponic system in terms of the period required to recover the initial investment, by accounting for regional costs of equipment, imports and labour. This indicator helps comparing economic performance across different locations, and assists the design process by adapting it to regional constraints.

\section{Chapter 3}

The model-based analysis and management strategy of the test INAPRO system in Abtshagen, Germany, showed that a decoupled aquaponic system can produce fish and tomato with fresh water use similar to commercial, independent RAS and greenhouse horticulture systems, and less than 1/10 of the $N$ fertilizer use (Table 3.4). However, simulated mineral concentrations of $\mathrm{NH}_{4}, \mathrm{Na}, \mathrm{Ca}$, and $\mathrm{Mg}$ reached or exceeded harmful levels for plants.

Actual tomato production observed in the Abtshagen system was lower than anticipated. The published model-based results (Reyes Lastiri et al., 2018), and the results obtained by Suhl et al. (2016), revealed that the low tomato production performance was a combination of the following factors:

The RAS loop only used one settling tank to remove solids. During the design stage of the system, tests on solids removal revealed that a single settling tank performed similar to the combination of mechanical filtration with a smaller settling tank (Kloas, 2016). The choice for solids removal was thus motivated by cost effectiveness in the RAS loop alone, without considering possible impacts on water quality requirements for the plants. As opposed to common RAS systems, which use a settling tank detached from the RAS loop to catch concentrated sludge from 
a mechanical filter (Fig. 2.1), the single settling tank in Abtshagen was part of the RAS loop (Fig. 3.1). This tank required frequent emptying to remove accumulated solids, as opposed to the feed-based strategy for water exchange proposed in Chapter 2. Furthermore, emptying the settling tank disrupts water quality by resuspension. Water removed from the settling tank was therefore diluted in nutrients, with variable and unpredictable concentrations. To prevent wasting the frequent water discharged from the RAS, a 3-chamber underground storage tank was used.

Lack of aeration in the 3-chamber underground storage tank. The large underground tank was used to accumulate the frequent water discharge from the RAS loop, and to further separate solids to prevent known damage to plant roots, and decreased oxygen levels in water. However, the accumulation of solids at the bottom of the tank resulted in anaerobic conditions, facilitating denitrification (conversion of $\mathrm{NO}_{3}^{-}$to gaseous $\mathrm{N}_{2}$ ), and thus to loss of $N$. Continuous aeration of the tank could have prevented the problem.

Mineral concentrations in fresh water. Fresh water was supplied from local ground water. Measurements reported by REWA (2017) showed relatively high concentrations of $\mathrm{Ca}, \mathrm{Mg}$ and $\mathrm{Na}$. Predicted concentrations of $\mathrm{Ca}$ and $\mathrm{Mg}$ from the RAS loop exceeded stress threshold levels for tomato plants, and predicted $\mathrm{Na}$ concentration were close to threshold stress levels. It was concluded that these minerals, present as conjugate acid-base pairs in e.g. phosphates or carbonates, acted as a $\mathrm{pH}$ buffer, preventing the need to add buffer minerals. However, mineral concentrations resulting from recirculating ground water were not considered when selecting fish feed. Therefore the presence of nutrients like $\mathrm{Na}$ in fish feed, exacerbated excess levels for tomato plants.

\section{Chapter 5}

The study on fish growth and excretions showed that there are large variations in experimental measurements reported in literature, which lead to large prediction uncertainties. The calibrated model reproduced most of the variations in fish growth and mass content of the digestive system. However, it did not reproduce the variations in ammonia excretion rates (Fig. 5.5).

An assessment of the model prediction uncertainties (Sections 5.4.2 and 5.4.3) helped identifying the following challenges and possible solutions:

Experimental measurement conditions. Controlled experimental conditions may result in water quality different from that present during common operation, which may have an impact on the system being studied. In particular, closed water recirculation in fish tanks, leads to the accumulation of ammonia, which in turn has an effect on subsequent ammonia excretion rates (Randall, 2011). Therefore, for future experimental research on aquaponic systems, it is suggested to use common growth 
conditions when taking measurements, e.g. fish tanks open to recirculating water, particularly when self-controlling feedback loops are expected.

Soluble excretions. The largest model prediction uncertainties on macronutrients in the soluble phase (Fig. 5.7). Therefore, towards improved accuracy of model predictions for macronutrients in recirculating aquacultural water, it is suggested to focus future experimental measurements on soluble $\mathrm{P}, \mathrm{Ca}$, and $\mathrm{Na}$.

\section{Summary of experiences on water and nutrient balances in aquaponics.}

Send water from fish to plants based on fish feeding schedules. This water exchange strategy stabilizes water quality for fish and improves predictability of nutrient concentrations for plants.

Use mechanical filtration and sedimentation in the fish loop. A sedimentation tank, separate from the fish loop, improves stability of nutrient concentrations available for plants.

Aerate water storage. Mismatched sizing of independently optimized fish and plant loops can be compensated with water storage. In that case, aeration is required to prevent loss of nitrogen through denitrification.

Select fish feed based on local fresh water quality. Local water may contain high concentrations of minerals like $\mathrm{Na}, \mathrm{Ca}$, and $\mathrm{Mg}$, which are harmful to plants, and thus limit the total amount of water that can be sent from fish to plants. Choosing fish feed based on local fresh water concentrations, helps balancing the needs of fish and plants.

Consider impacts across loops when optimizing them separately. A solution to improve one loop, may lead to problems on the second loop, and additional costs to solve the new problems.

\section{Research question 2.}

\section{What are the challenges and possible solutions in modelling nutri- ent balances and their uncertainties for aquaponics?}

Aquaponic systems have an intrinsic complexity due to the presence of multiple organisms in multiple water recirculation loops. This complexity results in large measurement uncertainties in experimental data, which hinder model calibration and prediction accuracy. Therefore, a modelling approach suitable for large uncertainties is required. Chapters 2 and 3 presented models for system-level water and nutrient balances in aquaponic systems. Chapter 4 presented a novel algorithm to calibrate mathematical models against limited experimental data under measurement uncertainties. Chapter 5 presented a model for nutrient balances in fish growth and excretions accounting for measurement and prediction uncertainties. 


\section{Chapters 2 and 3}

Based on the experiences from system-level modelling and simulation of aquaponics, it is suggested to consider the following challenges and proposed solutions.

Model implementation. The model-based study in Chapter 2 was implemented in Excel to facilitate communication. However, the time-step was small (15 min), and the simulation time was large (2-5 yr), which resulted in unstable Excel files. Therefore, for future research on modelling aquaponic systems, it is suggested to use simple spreadsheets for steady-state mass balances or an general overview of system dynamics (e.g. time step of $1 d$, for 1 yr simulation), and use programming languages such as Python or Matlab for the implementation of more detailed dynamics.

Modelling expertise. There is high multidisciplinarity involved in aquaponic systems. Combining knowledge on plant growth, greenhouse climate, fish production, mathematical modelling and programming, results in a slow learning curve that should be considered when performing research that combines modelling and experimental studies. Furthermore, in systems with large uncertainties, modelling decisions seem to have an influence on the simulation results (Melsen et al., 2019), such that the modellers themselves can be considered an additional source of uncertainty. Even more when considering that doctoral research is part of the scientific formation of the modeller. Therefore, it is necessary to report carefully all modelling assumptions and decisions, understanding that perfectly reported, universal modelling decisions, is an ideal aim, not a guaranteed standard.

Complexity. In this thesis, Chapter 2 focused on modelling water and $N$ balances, and Chapter 3 extended the balances to several macronutrients. This approach, with a sequential increase in complexity, facilitated the modelling process, but it adds to the time required in the development of modelling expertise. It is therefore important to focus on a specific topic (e.g. nurient balances, energy balances, or water quality control). For future research on nutrient balances, it is suggested to expand the models of macronutrients presented in this thesis, considering their bioavailability for plant uptake. In particular, modelling the competition that exists between some ions, such as $\mathrm{Na}^{+}$inhibition of $\mathrm{Ca}^{+}$and $\mathrm{K}^{+}$uptake (Volkmar et al., 1998). These competition process could be modelled as a combination of ideal electrical components (resistor, capacitor and inductor), similar to the nerve conduction model proposed by Mattheij and Molenaar (2002).

Modelling loops. The implementation of numerical methods with a single time step for the sequential solution of components in multiple loops of aquaponic systems, may easily lead to oscillations in the model predictions. These oscillations are purely numerical (Section 3.4.4). In Chapter 3, these numerical oscillations were tackled using a low-pass filter on the simulation results. However, this approach 
may lead to the removal of physical oscillations in components with fast or multiple dynamics, such as the biofilter in a RAS loop. Therefore, it is suggested to use individual time steps per component, and to include models for the dynamics of mixing in tanks, to prevent large numerical oscillations from discrete changes, with a subsequent analysis of the parameter uncertainty introduced by the mixing terms in the mass balances.

\section{Chapters 4 and 5}

Large uncertainties have been observed in the components of aquaponic systems (see for example Fig. 5.2). A set-membership algorithm for the calibration of complex models under uncertainty was developed in Chapter 4 . The objective of this set-membership approach was to represent all unknown sources of errors and measurement uncertainties as uncertainty in the model parameters.

The algorithm was used in Chapter 5 for the calibration of a model for fish growth and macronutrient excretions. The model showed that it is possible to represent the dynamics of growth and excretions based on simple mass balances. The model explained most of the variations in experimental data for fish growth and mass content in the digestive system. However, it did not explain the variations in experimental TAN excretions (Fig. 5.5).

Based on the experiences from the algorithm development and its implementation in the modelling process, it is suggested to consider the following challenges and proposed solutions:

Random sampling. The algorithm was first developed in Chapter 4 using default random sampling routines in NumPy 1.14 (The SciPy community, 2018a), without specifying the seed. Therefore, multiple runs were required to verify the consistency of simulation results (Table 4.2). The algorithm was further developed in Chapter 5 for its implementation in the fish model calibration, using sampling routines from Numpy 1.17, which use a direct specification of the seed for random number generators (The SciPy community, 2021). A specific seed value facilitates reproducibility. Notice however that a seed value must still be specified, because the default value (None) returns unpredictable results.

Multiple outputs and multiple time scales. Using multiple model outputs helps improving identifiability, and decreasing prediction uncertainty (Sections 5.4.1 and 5.4.2) (Guillaume et al., 2019). However, aquaponic systems often present different time scales across model outputs, determined by the feasibility of experimental measurements. For example, the fish model studied in Chapter 5 has time scales spanning several months for fish growth, and several hours for mass content in the digestive system and for TAN excretion rate (Figs. 5.2). Default routines in programming languages are often based on the assumption of equal time scales for 
all model outputs. Implementing and simulating multiple model outputs with different time scales and different measurement instants is not trivial. Simultaneous analysis of all essential time scales, followed by quasi-steady state simulation when possible, help improving prediction accuracy and should be considered in future research.

\subsection{A framework for modelling growth and nutrient bal- ances in biological production systems}

The experiences gained from the models developed in this thesis can be extended from the specific field of aquaponics, to propose a general framework for modelling growth and nutrient balances in biological production systems.

First, it is suggested to construct the mathematical model based on the combination of the approaches mentioned in the General Introduction (Section 1.3), with the following characteristics and advantages:

- System dynamics: The model should be based on a systems of differential equations and supporting algebraic equations. Common solver algorithms, such as Euler-Forward, can then be applied to solve the system of equations. It is suggested to use a constant time step within each component, to facilitate computations between total mass and flow rates under discrete changes.

- Semi-physical: The model structure should follow a grey-box approach, combining prior knowledge in the form of a mathematical models, with experimental measurements. The model should describe simple mass balances, following first principles, but maintaining a system-level perspective oriented to measurable model outputs (i.e. neglecting underlying physiological processes at cellular level). Model parameters can then be calibrated against experimental data. The variables and model parameters should have a physical meaning, which provides the means to gain further understanding from the model predictions and their comparison against experimental measurements.

- Simplification: The model structure should be limited to measurable processes in common operation conditions. Identify the most relevant model parameters using a sensitivity analysis. Calibrate only the most relevant model parameters. Analyse the model prediction uncertainty, and add complexity only when it can improve prediction accuracy.

- Modularity: The model structure should describe the system components or process units as sub-models (modules), which are represented by their own 
system of equations. In this way, each module can be solved with their own time step, accounting for their own (fast or slow) dynamics. The system-level simulation can then be performed with its own general time step.

- Sequential: The system modules should be solved in sequence (as opposed to in parallel), interpolating information from preceding modules as inputs, and providing inputs for subsequent modules. In this way, differences in the dynamics across modules can be tackled independently, without relying on computer-defined varying time steps that cannot be easily accessed by the modeller. Physical loops in the system can be tackled by convergence criteria or, more simply, by means of a system level time step smaller than the expected recirculation time.

- Deterministic with stochastic elements: The model should predict single trajectories, while accounting for the large variations inherent to biological systems. It is suggested to express all errors and measurement uncertainties as parameter uncertainty. This parameter uncertainty can be first expressed in terms of a feasible parameter set (FPS). Subsequently, and given increasing experimental information, the parameter uncertainty can be expressed in terms of a standard error and a probability density function $\left(\theta_{p} \pm \sigma_{p} \mid p d f\right)$. In this way, mechanistic single trajectories allow for reproducibility of model results, while the stochastic parameter uncertainty, with its corresponding model prediction uncertainty, provide a tool to describe variations due to (unknown) underlying processes (e.g. phenotypical variations between individual organisms).

Once the mathematical model has been constructed, it can be analysed and further improved with the following sequence of steps (Fig. 6.1).

1. Model evaluation. Compare the behaviour of model predictions against experimental data, for as many model outputs as available from experimental data, using the corresponding time scales. Constrain model parameters to sensible physical values, supported by prior information from literature. Select unknown or unconstrained parameters for subsequent calibration.

2. All-at-a-time (AAT) sensitivity analysis. Identify the most relevant parameters to further simplify the model. Use AAT methods (as opposed to oneat-a-time, OAT), which are better suited for non-linear models, often used to describe the complexity of biological production systems.

3. Calibration under uncertainty. Biological models often involve limited data with large measurement uncertainties. If that is the case, one possible method 


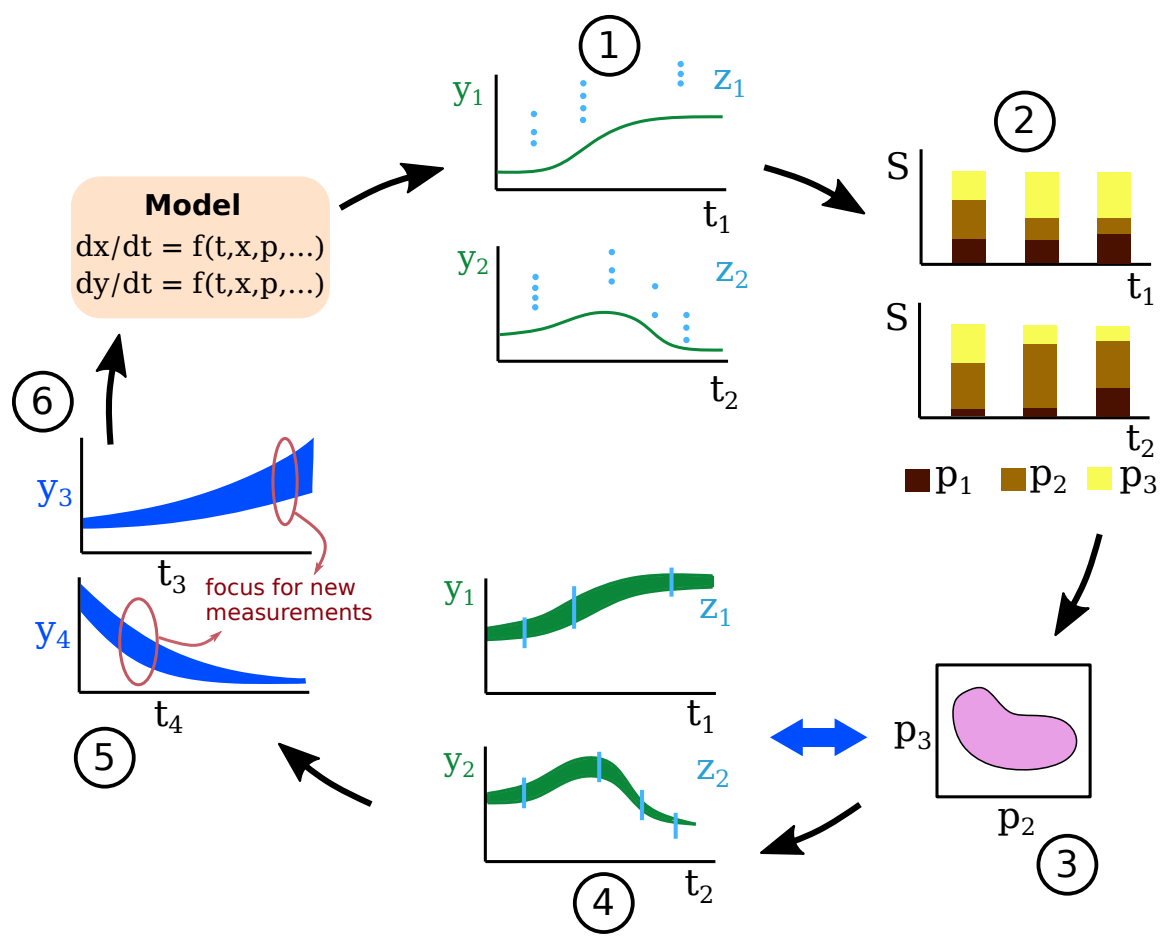

FIGURE 6.1: A proposed framework for modelling biological production systems under uncertainty.

for model calibration is set-membership. For set-membership calibration, generate data bounds by combining information from existing measurements, with the expected behaviour proposed in the model. If sufficient data is available, statistical methods like least-squares or maximum likelihood estimation can be used.

4. Evaluate reproduction of data variation. Assess deviations between model prediction uncertainty and experimental data. Identify possible causes of these deviations to support further model development. Increase model complexity only when the introduction of new parameters can improve the reproduction of experimental uncertainties.

5. Evaluate uncertainty propagation. Analyse the propagation of uncertainty to other model outputs and other system components. Possible methods are: a) linear propagation, b) Monte Carlo simulations from parameter standard deviations and correlation matrices, and c) Monte Carlo simulations from the feasible parameter set (FPS) identified by set-membership. Identify model predictions with the largest uncertainties to focus future experimental measurements on those variables.

6. Data assimilation. Use new experimental data to improve the structure of the model, and recalibrate it. 
For this framework, it is suggested to start from a simple model structure, and introduce complexity only when deemed useful to decrease prediction uncertainty. In contrast, approaches like model order reduction, start from a complex initial structure with multiple state variables, and decrease this complexity in subsequent steps by means of e.g. numerical approximations. Suggested applicability cases for these two contrasting approaches are:

a) model simplicity for system-level studies aimed at design, management or control (e.g. yield, resource efficiency),

b) model complexity for detailed understanding of underlying mechanisms (e.g. spatial transfer in mixing, high frequency dynamics in ion exchange between water and organisms).

In the context of model prediction uncertainty, the suggested framework can be understood in terms of the Bayes theorem. Starting from set-membership calibration for limited data under large measurement uncertainties, every new measurement updates the prior knowledge by possibly decreasing the error bounds. Eventually, the number of measurements should support the introduction of statistical methods in subsequent calibration. For future research, it is suggested to determine the number of measurements that could be considered sufficient to switch from error bounds to a statistical description of the measurement errors.

Finally, the suggested sequence of steps can be applied to the context of precision farming, combining prior knowledge described as a mathematical model, and insitu measurements to control the system and update the model, with the objectives of improved resource efficiency, animal welfare, and quality of life.

\subsection{Socio-economic challenges for aquaponics and lo- cal food production}

The global context of food production, fisheries, and their impacts, was presented in the General Introduction (Section 1.1). Aquaponics was presented as an alternative local food production method, with potential for improved sustainability, focused on closing water and nutrient cycles in a single resilient system (Section 1.2). However, in addition to the technical challenges in closing cycles, the development of aquaponics faces socio-economic challenges.

The main problem in global food production and trade can be summarized in the externalization of its impacts, due to a general disconnection between the general public and the food production sector. With the development of industrial agriculture, people migrated from rural areas to the cities motivated by better opportunities 
for a stable income and quality of life. However, this continued migration led to the abandonment of rural areas and eventual decrease of basic services in the countryside, leading to a positive feedback effect that further increased this migration flow. As a result, the global context of food production involves large inequalities and external impacts which remain out of sight of the consumers and, therefore, unsolved. These externalities and their limited visibility have been exacerbated by global trade (Fig. 6.2).

Aquaponics has been identified as a potential solution towards local, sustainable food production. For example, the EU recommends promoting innovative production processes like aquaponics, to ensure sustainability in the future of European aquaculture (European Union, 2015).

However, aquaponics is an emerging technology. It provides water savings and high productivity, but it is limited by high knowledge requirements, initial investments, and high utility costs. As a result, in low-income countries, aquaponics is mainly motivated by subsistence of the farmers themselves with possible benefits on dietary diversity. The financial benefits of aquaponics remain low, and the lack of supporting policies appear to constrain its potential (Poulsen et al., 2015). Similarly, in the EU there are multiple legal barriers, such as having fish and plant production

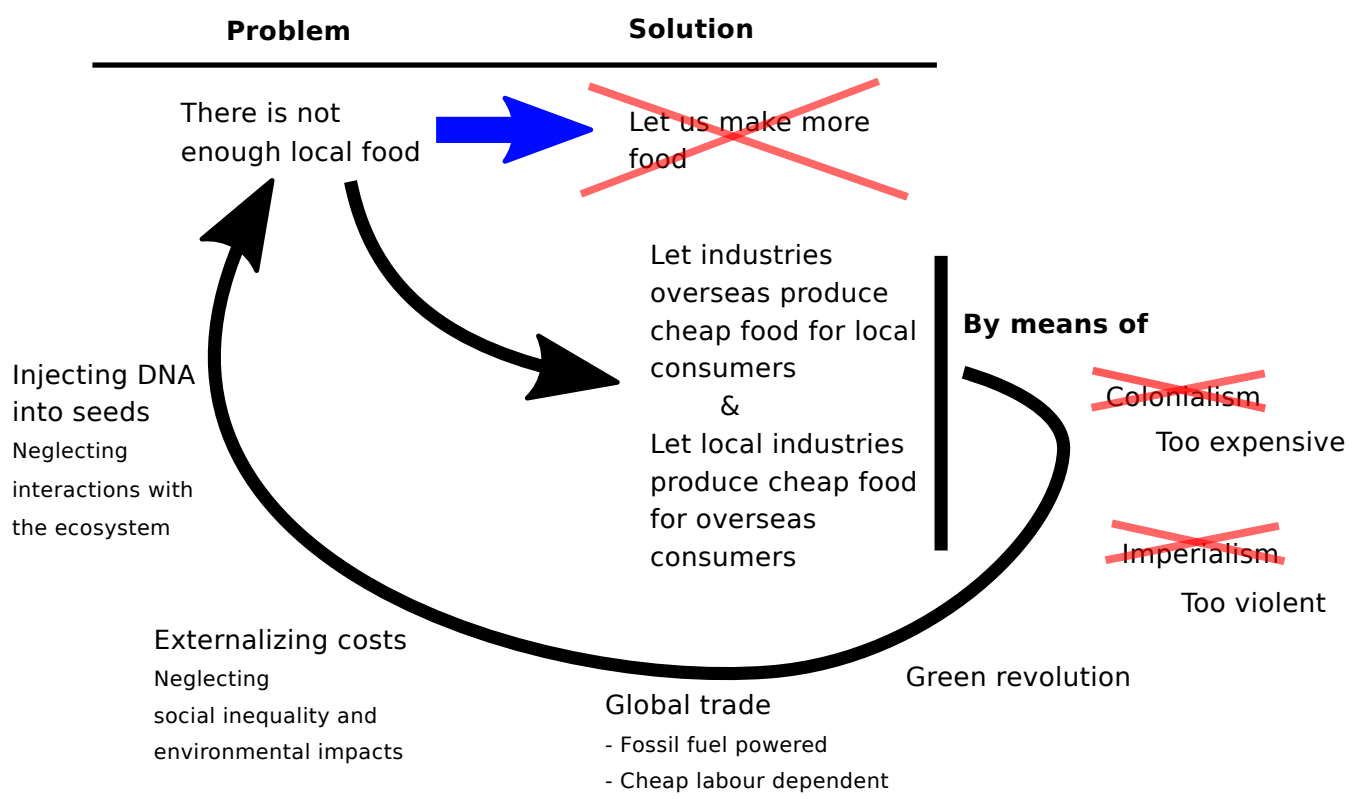

FIGURE 6.2: Summary of the modern development of global food production and its impacts. Information from Galeano (1971), Sachs (2008), Easterly (2006), Mulder (2017), and Van Santen et al. (2010). Local food production has long been identified as necessary yet insufficient. It has concentrated in areas with high productivity to help solving short-term demand. Most environmental and social impacts take place far from the consumers, resulting in an increase of negative impacts, which has not yet solved the identified insufficiencies and inequalities in the access to food. 
regulated by different governmental bodies, and the lack of legal access to farmer status and its accompanying social benefits due to the small size of ventures (Joly et al., 2015). These challenges are common to all urban agriculture methods.

In the Netherlands, the development of aquaponics has also been hindered by the lack of communication between researchers and entrepreneurs, and an environment of secrecy and competition among entrepreneurs (Wiegand, 2019). Facing similar challenges, but aimed at limiting impacts on biodiversity, Clay (2010) argues that significant changes can be accomplished by targetting large players, instead of small initiatives or consumers, focusing attention on strategic items with the largest impacts across the production chain. Therefore, it is important to 1) identify the main opportunities for the development of strategic components of aquaponic systems, and 2) establish adequate communication channels for this knowledge.

1) Strategic components. Presently, aquaponic ventures mostly rely on revenue from plant production. Love et al. (2015), proposed that the reason could be larger biomass conversion at higher rates for plants than for fish. Additionally, it could be argued that greenhouse horticulture is a better established and more resilient technology than RAS. In RAS, perturbations such as disease can easily spread in the recirculating water and affect all organisms living within, resulting in the loss of an entire production cycle. Furthermore, as shown in Section 5.4.3, there are large uncertainties on the nutrients available from fish to fertilize plants (Fig. 5.7). As a result, engagement of horticultural producers in aquaculture involves larger risks than the opposite alternative. Therefore, for future research, it is suggested to perform an economic risk assessment. If risks are indeed more sensitive to the RAS production, it is suggested to provide incentives for RAS farmers to implement hydroponics. Such incentives could be supported by the benefits from lower water and nutrient discharge (to remain within permissible levels), and the inherent diversification of revenue streams. Revenue diversification helps preventing risks associated to e.g. subsidy dependence, which recently impacted inland aquaculture in the Netherlands (Visserijnieuws, 2014).

2) Communication. It is necessary to continue using open access publications and open-source software. Additionally, it is necessary and to improve our engagement in science communication with the general public. Currently, higher education institutions focus scientific output on peer-review publications. Alternatives for scientific output directed to the general public must become an institutional part in the formation of young researchers. This communication is important for aquaponics and local food production methods in particular, and for emerging technologies in general. 
An additional benefit from aquaponics, in the context of urban agriculture, is the possibility for improved knowledge on the real costs of production. Clay (2010) argues that consumers can never have perfect knowledge on the real production costs of their food, particularly in the current global trade context. As a result, large externalities only become known when it is too late to implement significant changes in the production chain. These externalities are simply too far from the consumers to notice them in their early stages. For example, environmental and antibiotic contaminants in farmed salmon (Yeats et al., 2005; Armstrong et al., 2005), water scarcity exacerbating the poverty trap of farmers in Chile for avocado production and export to Europe (O'Neill, 2014), cruel horse blood farming in Argentina and Uruguay for PMSG hormone use in pig farming in Europe (Sagener, 2017). Aquaponics and other local and urban food production methods can help preventing such negative externalities, by means of community engagement and a more direct flow of information between producers and consumers.

It is important to maintain a critical perspective on further developments of aquaponics. Currently, food production in high-income countries aims for automation, for example, in the context of precision farming, which bring higher resourceefficiency and lower hard-labour for an improved quality of life of the farmers. However, these developments could potentially decrease community engagement in food production even further, maintaining current externalities and adding new ones. For example, hi-tech components used in precision farming have high environmental costs and, in some cases, very high human costs related to the mining of materials required, such as coltan (Ayres, 2012; Moran et al., 2015). In a different example, small European farmers have raised concerns regarding data ownership of precision farming technologies (WUR, 2019).

Automation in agriculture aims at improving quality of life by replacing human labour, but its impacts must be kept in sight. And a few extra hands in agriculture wouldn't hurt. Migration from rural to urban areas was motivated by the lack of access to basic services, but technological advances in transportation and communications have now facilitated that access. Therefore, at the time of burnouts challenging the promise for quality of life, some of us could go back to the agricultural sector, whether it be within the calm of a rural setting or within the comfort of a city, we can all benefit from the opportunity to engage in local food production "with our own two hands". 


\section{Bibliography}

Allaman, Ivan Bezerra et al. (2013). "Weight and morphometric growth of different strains of tilapia (Oreochromis sp)". In: Revista Brasileira de Zootecnia 42.5, pp. 305-311.

Armstrong, S. M., B. T. Hargrave, and K. Haya (2005). “Antibiotic Use in Finfish Aquaculture: Modes of Action, Environmental Fate,and Microbial Resistance". In: Environmental Effects of Marine Finfish Aquaculture. Ed. by Barry T. Hargrave. Berlin, Heidelberg: Springer Berlin Heidelberg, pp. 341-357. DOI: 10 . 1007/b136017. Ayres, Christopher J (2012). “The international trade in conflict minerals: Coltan". In: critical perspectives on international business.

Barber, C. Bradford, David P. Dobkin, and Hannu Huhdanpaa (1996). “The Quickhull Algorithm for Convex Hulls". In: ACM Trans. Math. Softw. 22.4, pp. 469-483. ISSN: 0098-3500. DOI: 10.1145/235815.235821.

Belal, Ibrahim EH (2005). "A review of some fish nutrition methodologies". In: Bioresource technology 96.4, pp. 395-402. DOI: 10.1016/j. biortech.2003.11.030.

Bergheim, Asbjørn, Simon J Cripps, and Helge Liltved (1998). "A system for the treatment of sludge from land-based fish-farms". In: Aquatic Living Resources 11.4, pp. 279-287. DOI: 10.1016/S0990-7440 (98)80013-2.

Bernstein, Sylvia (2011). Aquaponic gardening: a step-by-step guide to raising vegetables and fish together. New society publishers.

Birta, Louis G and Gilbert Arbez (2013). Modelling and simulation. Exploring dynamic system behaviour. 2nd ed. Springer.

Borgognone, Daniela et al. (2013). "Effect of nitrogen form and nutrient solution $\mathrm{pH}$ on growth and mineral composition of self-grafted and grafted tomatoes". In: Scientia horticulturae 149, pp. 61-69. DOI: 10.1016/ j . scienta.2012.02.012.

Breitburg, Denise et al. (2018). "Declining oxygen in the global ocean and coastal waters". In: Science 359.6371. DOI: 10.1126/science . aam7240.

Brown, Lester R. (2001). "Erradicating hunger: a growing challenge. A Worldwatch Institute Report on Progress Toward a Sustainable Society". In: Brown, Lester R., Christopher Flavin, and Hilary French. State of the World, 2001. New York and London: W. W. Norton \& Company Inc. and W. W. Norton \& Company, Ltd.

Buitelaar, Rudolf M and Ramón Padilla Pérez (2000). "Maquila, economic reform and corporate strategies". In: World Development 28.9, pp. 1627-1642. DOI: 10 . 1016/S0305-750X (00)00041-3. 
Canale, Raymond P and James E Breck (2013). "Comments on proper (and improper) solutions of bioenergetic equations for modeling fish growth". In: Aquaculture 404, pp. 41-46. DOI: 10.1016/j . aquaculture.2013.04.009.

Carvajal, Micaela, Vicente Martinez, and Antonio Cerda (1999). "Influence of magnesium and salinity on tomato plants grown in hydroponic culture". In: Journal of plant nutrition 22.1, pp. 177-190. DOI: 10.1080/01904169909365616.

Cerone, Vito et al. (2014). "A unified framework for solving a general class of conditional and robust set-membership estimation problems". In: IEEE Transactions on Automatic Control 59.11, pp. 2897-2909. DOI: 10.1109/TAC. 2014.2351695.

Cerozi, B.S. and K. Fitzsimmons (2017). "Phosphorus dynamics modeling and mass balance in an aquaponics system". In: Agricultural Systems 153, pp. 94-100. DOI: 10.1016/j.agsy.2017.01.020.

Chen, Shulin, David E Coffin, and Ronald F Malone (1997). "Sludge production and management for recirculating aquacultural systems". In: Journal of the World Aquaculture Society 28.4, pp. 303-315. DOI: 10.1111/ j.1749-7345 . 1997 . tb00278. $\mathrm{x}$.

Clay, Jason (2010). How big brands can help save biodiversity. TED. URL: https : //www . youtube. $\mathrm{com} /$ watch? $\mathrm{v}=\mathrm{jcp} 5 \mathrm{vvxtEaU}$.

- (2013). World agriculture and the environment: a commodity-by-commodity guide to impacts and practices. Island Press.

Coche, AG (1967). "Fish culture in rice fields a world-wide synthesis". In: Hydrobiologia 30.1, pp. 1-44. DOI: 10 .1007/BF00135009.

Cripps, Simon J and Asbjørn Bergheim (2000). "Solids management and removal for intensive land-based aquaculture production systems". In: Aquacultural engineering 22.1-2, pp. 33-56. DOI: 10.1016/S0144-8609 (00)00031-5.

De Gelder, A, E Heuvelink, and JJG Opdam (2005). “Tomato yield in a closed greenhouse and comparison with simulated yields in closed and conventional greenhouses". In: Acta Horticulturae 2005.691, pp. 549-552. DOI: 10 . 17660/ActaHortic . 2005.691 .65$.

DeLong, Dennis P, Thomas Losordo, and James Rakocy (2009). Tank culture of tilapia. Southern Regional Aquaculture Center. URL: https : / / wkrec . ca . uky . edu / files/tilapiatankculture.pdf (visited on 05/2021).

Diniz, Paulo S.R. (2013). Adaptive Filtering. Algorithms and Practical Implementation. 4th ed. Springer. DOI: 10.1007/978-1-4614-4106-9.

Dominguez, Miriam et al. (2004). "Impact of different environmental factors on the circulating immunoglobulin levels in the Nile tilapia, Oreochromis niloticus". In: Aquaculture 241.1, pp. 491-500. DOI: 10.1016/j . aquaculture.2004.06.027.

Easterly, William (2006). The White Man's Burden: Why the West's Efforts to Aid the Rest Have Done so Much Ill and so Little Good. 
Ebeling, J M and B Vinci (2006). Recirculating aquaculture systems, short course. Solids capture. University of Arizona. College of Agriculture and Life Sciences.

El-Shafai, Saber A et al. (2004). "Chronic ammonia toxicity to duckweed-fed tilapia (Oreochromis niloticus)". In: Aquaculture 232.1-4, pp. 117-127. DOI: 10 . 1016 / S0044-8486 (03) 00516-7.

European Union (2015). "Opinion of the European Committee of the Regions - the future of European aquaculture". In: Official Journal of the European Union 423 (C), pp. 20-23. URL: https : / / eur - lex . europa . eu / legal - content / EN / TXT / PDF / ?uri=CELEX : 52015IR2712\& from=EN (visited on 05/2021).

FAO (2011). The state of world's land and water resources for food and agriculture. Managing systems at risk. Rome, London: FAO-UN, Earthscan.

- (2016). The State of World Fisheries and Aquaculture. Contributing to food security and nutrition for all. Rome.

- (2018a). Oreochromis niloticus. Fisheries and aquaculture department. URL: http: //www . fao .org/fishery/culturedspecies/Oreochromis_niloticus/en (visited on $05 / 2021$ ).

- (2018b). The State of World Fisheries and Aquaculture 2018 - Meeting the sustainable development goals. Rome: License: CC BY-NC-SA 3.0 IGO.

- (2021). Aquaculture topics and activities. Aquaculture resources. URL: http: //www . fao.org/fishery/countrysector/naso_netherlands/en (visited on 05/2021).

Forchino, A.A. et al. (2017). "Aquaponics and sustainability: The comparison of two different aquaponic techniques using the Life Cycle Assessment (LCA)". In: Aquacultural Engineering 77, pp. 80-88. DOI: 10.1016/j . aquaeng. 2017.03.002.

Føre, Martin et al. (2018). "Precision fish farming: A new framework to improve production in aquaculture". In: biosystems engineering 173, pp. 176-193. DOI: 10. 1016/j. biosystemseng. 2017.10.014.

Fritzsche, Eva et al. (2018). "A validation and comparison study of new, compact, versatile optodes for oxygen, $\mathrm{pH}$ and carbon dioxide in marine environments". In: Marine Chemistry 207, pp. 63-76. DOI: 10.1016/j . marchem. 2018.10.009.

Galeano, Eduardo (1971). Las venas abiertas de América Latina. Siglo XXI.

Gallardo, $\mathrm{M}$ et al. (2009). "Simulation of transpiration, drainage, $\mathrm{N}$ uptake, nitrate leaching, and $\mathrm{N}$ uptake concentration in tomato grown in open substrate". In: Agricultural Water Management 96.12, pp. 1773-1784. DOI: 10.1016/ j . agwat . 2009.07.013.

Goddek, Simon and Karel J Keesman (2018). “The necessity of desalination technology for designing and sizing multi-loop aquaponics systems". In: Desalination 428, pp. 76-85. DOI: 10.1016/j.desal.2017.11.024. 
Goddek, Simon and Oliver Körner (2019). "A fully integrated simulation model of multi-loop aquaponics: A case study for system sizing in different environments". In: Agricultural Systems 171, pp. 143-154. DOI: 10 . 1016/ j . agsy . 2019. 01.010.

Goddek, Simon et al. (2016). "Navigating towards decoupled aquaponic systems: A system dynamics design approach". In: Water 8.7, p. 303. DOI: 10.3390/w8070303.

Gómez-Peñaranda, José Ader and Laura Cristina Clavijo-Restrepo (2012). “Determination of the stomach emptying time of tilapia Oreochromis sp. using different weekly feeding frequencies and starvation". In: Acta Agronómica 61.3, pp. 239243.

Grassini, Patricio, Kent M Eskridge, and Kenneth G Cassman (2013). “Distinguishing between yield advances and yield plateaus in historical crop production trends". In: Nature communications 4, p. 2918. DOI: 10.1038/ncomms3918.

Guillaume, Joseph HA et al. (2019). "Introductory overview of identifiability analysis: A guide to evaluating whether you have the right type of data for your modeling purpose". In: Environmental Modelling E Software 119, pp. 418-432. DOI: 10.1016/j.envsoft.2019.07.007.

Gullian-Klanian, MAAC and C Arámburu-Adame (2013). “Performance of Nile tilapia Oreochromis niloticus fingerlings in a hyper-intensive recirculating aquaculture system with low water exchange". In: Latin American Journal of Aquatic Research 41.1, pp. 150-162.

Hamilton, M Coreen et al. (2005). "Lipid composition and contaminants in farmed and wild salmon". In: Environmental science \& technology 39.22, pp. 8622-8629. DOI: 10.1021 /es050898y.

Hashimoto, Yasushi et al. (1993). The computerized greenhouse: automatic control application in plant production. Academic Press. DOI: 10 .1016/C2009-0-03304-2.

Herman, Jon and Will Usher (2017). "SALib: an open-source Python library for sensitivity analysis". In: The Journal of Open Source Software 2.9. DOI: 10.21105/ joss . 00097.

Herman, Jon et al. (2019). SALib, Sensitivity Analysis Library in Python. URL: https: //salib.readthedocs.io/en/latest/ (visited on 05/2021).

Heuvelink, Ep (1996). "Dry matter partitioning in tomato: validation of a dynamic simulation model". In: Annals of botany 77.1, pp. 71-80. DOI: 10.1006/anbo . 1996. 0009.

- (1999). "Evaluation of a dynamic simulation model for tomato crop growth and development". In: Annals of Botany 83.4, pp. 413-422. DOI: 10.1006/anbo 1998. 0832.

Hites, Ronald A et al. (2004). "Global assessment of organic contaminants in farmed salmon". In: Science 303.5655, pp. 226-229. DOI: 10.1126/science.1091447. 
Hoekstra, Arjen Y and Mesfin M Mekonnen (2012). "The water footprint of humanity". In: Proceedings of the national academy of sciences 109.9, pp. 3232-3237. DOI: 10.1073/pnas. 1109936109.

Holmberg, Andrea (1982). "On the practical identifiability of microbial growth models incorporating Michaelis-Menten type nonlinearities". In: Mathematical Biosciences 62.1, pp. 23 -43. ISSN: 0025-5564. DOI: 10. 1016/0025-5564 (82) 90061-X.

INAPRO (2014). Innovative aquaponics for professional applications. URL: http: // www . inapro-project. eu/ (visited on 05/2021).

Jancke, Ralf (2016). Personal communication. INAPRO.

Jaulin, Luc and Eric Walter (1993). "Set inversion via interval analysis for nonlinear bounded-error estimation". In: Automatica 29.4, pp. 1053 -1064. DOI: 10 . 1016 / 0005-1098 (93) 90106-4.

Jaxa-Rozen, Marc and Jan Kwakkel (2018). “Tree-based ensemble methods for sensitivity analysis of environmental models: A performance comparison with Sobol and Morris techniques". In: Environmental Modelling \& Software 107, pp. 245-266. DOI: 10.1016/j.envsoft. 2018.06.011.

Joly, Agnes, Ranka Junge, and Tamas Bardocz (2015). "Aquaponics business in Europe: some legal obstacles and solutions". In: Ecocycles 1.2, pp. 3-5. DOI: 10 . 19040/ecocycles.v1i2.30.

Karimanzira, Divas et al. (2016). "Dynamic modeling of the INAPRO aquaponic system". In: Aquacultural Engineering 75, pp. 29 -45. DOI: 10 .1016/ j . aquaeng . 2016.10.004.

Kearney, John (2010). “Food consumption trends and drivers". In: Philosophical Transactions: Biological Sciences 365.1554, pp. 2793-2807. DOI: 10 . 1098/rstb . 2010. 0149.

Keesman, Karel (1990). "Membership-set estimation using random scanning and principal component analysis". In: Mathematics and Computers in Simulation 32.56, pp. 535-543. DOI: 10.1016/0378-4754 (90)90009-8.

Keesman, Karel and Gerrit Van Straten (1990). "Set membership approach to identification and prediction of lake eutrophication". In: Water Resources Research 26.11, pp. 2643-2652. DOI: 10.1029/WR026i011p02643.

Keesman, Karel J (2011). System identification: an introduction. London: Springer Science \& Business Media.

Kelley, William Terry et al. (2010). Commercial tomato production handbook. University of Georgia.

Khoury, Colin K et al. (2014). "Increasing homogeneity in global food supplies and the implications for food security". In: Proceedings of the National Academy of Sciences 111.11, pp. 4001-4006. DOI: 10.1073/pnas . 1313490111.

Kloas, Werner (2016). INAPRO modelling meeting. Personal communication. 
Kloas, Werner et al. (2015). "A new concept for aquaponic systems to improve sustainability, increase productivity, and reduce environmental impacts". In: Aquaculture environment interactions 7.2, pp. 179-192. DOI: 10.3354 /aei00146.

KNMI, Koninklijk Nederlands Meteorologisch Instituut (2014). Daggegevens van het weer in Nederland. URL: http : / / ww . knmi . nl / klimatologie / daggegevens / download. html (visited on 05/2021).

Komosa, A et al. (2011). "Comparison of yield, nutrient solution changes and nutritional status of greenhouse tomato grown in recirculating and non-recirculating nutrient solution systems". In: Journal of plant nutrition 34.10, pp. 1473-1488. DOI: 10.1080/01904167.2011.585204.

Kooijman, Bas and SALM Kooijman (2010). Dynamic energy budget theory for metabolic organisation. 3rd. Cambridge University Press.

Langer, Vibeke (Aug. 1996). "Insect-crop interactions in a diversified cropping system: parasitism by Aleochara bilineata and Trybliographa rapae of the cabbage root fly, Delia radicum, on cabbage in the presence of white clover". In: Entomologia Experimentalis et Applicata 80.2, pp. 365-374. DOI: 10 . 1111 / j . 1570 7458.1996.tb00949.x.

Lewis, William M et al. (1978). “Use of hydroponics to maintain quality of recirculated water in a fish culture system". In: Transactions of the American Fisheries Society 107.1, pp. 92-99. DOI: 10.1577/1548-8659 (1978) 107<92:UOHTMQ>2. 0 . C0; 2.

Love, David C et al. (2015). "Commercial aquaponics production and profitability: Findings from an international survey". In: Aquaculture 435, pp. 67-74. DOI: 10 . $1016 / j$. aquaculture.2014.09.023.

Madan, Hennadii (2017). Sampling uniformly in a multidimensional ring without rejection. URL: https : / / stackoverflow . com / questions / 47472123 / sample uniformly-in-a-multidimensional-ring-without-rejection/47492146 (visited on $05 / 2021)$.

Martins, CIM et al. (2010). "New developments in recirculating aquaculture systems in Europe: A perspective on environmental sustainability". In: Aquacultural Engineering 43.3, pp. 83-93. DOI: 10.1016/j . aquaeng.2010.09.002.

Marvel, Skylar W and Cranos M Williams (2012). "Set membership experimental design for biological systems". In: BMC systems biology 6.1, p. 21. DOI: 10 .1186/ 1752-0509-6-21.

Mattheij, Robert and Jaap Molenaar (2002). Ordinary differential equations in theory and practice. SIAM.

Melsen, Lieke A. et al. (2019). "Subjective modeling decisions can significantly impact the simulation of flood and drought events". In: Journal of Hydrology 568, pp. 1093-1104. DOI: 10.1016/j.jhydrol.2018.11.046. 
Metcalf-Eddy et al. (2014). Wastewater engineering: treatment and resource recovery. 5th ed. New York, NY: McGraw-Hill.

Milanese, Mario et al. (1996). Bounding Approaches to System Identification. New York, NY: Plenum Press.

Mocenni, C and A Vicino (2006). "Modelling ecological competition between seaweed and seagrass: A case study". In: IFAC Proceedings Volumes 39.1, pp. 732737. DOI: 10 . 3182/20060329-3-AU-2901.00114.

Mohtar, RH, DR Buckmaster, and SL Fales (1997). “A grazing simulation model: GRASIM A: Model development". In: Transactions of the ASAE 40.5, pp. 14831493. DOI: $10.13031 / 2013.21370$.

Montanhini Neto, Roberto and Antonio Ostrensky (2015). "Nutrient load estimation in the waste of Nile tilapia Oreochromis niloticus (L.) reared in cages in tropical climate conditions". In: Aquaculture Research 46.6, pp. 1309-1322. DOI: doi : 10 . 1111/are. 12280.

Moran, Daniel et al. (2015). “Global supply chains of coltan: a hybrid life cycle assessment study using a social indicator". In: Journal of Industrial Ecology 19.3, pp. 357-365. DOI: $10.1111 /$ jiec. 12206.

Mulder, Karel (2017). Sustainable development for engineers: A handbook and resource guide. Routledge.

Naegel, Ludwig CA (1977). "Combined production of fish and plants in recirculating water". In: Aquaculture 10.1, pp. 17-24. DOI: 10 .1016/0044-8486 (77) 900291.

Nederhoff, Elly and Cecilia Stanghellini (2010). "Water Use Efficiency of Tomatoes". In: Practical Hydroponics and Greenhouses 115, pp. 52-59.

Norton, J.P. (1987). "Identification and application of bounded-parameter models". In: Automatica 2.4, pp. 497 -507. ISSN: 0005-1098. DOI: 10 . 1016/0005-1098(87) 90079-3.

Nurulhuda, K., P.C. Struik, and K.J. Keesman (2017). “Set-membership estimation from poor quality data sets: Modelling ammonia volatilisation in flooded rice systems". In: Environmental Modelling E Software 88, pp. 138 -150. ISSN: 13648152. DOI: $10.1016 / j$. envsoft. 2016.11.002.

Obirikorang, Kwasi Adu et al. (2015). “Digestibility and postprandial ammonia excretion in Nile tilapia (Oreochromis niloticus) fed diets containing different oilseed by-products". In: Aquaculture international 23.5, pp. 1249-1260. DOI: 10 . 1007 / s10499-015-9881-z.

Obirikorang, Kwasi Adu, Stephen Amisah, and Peter Vilhelm Skov (2017). "Effect of some common West African farm-made feeds on the oxygen consumption and ammonia excretion rates of Nile tilapia, Oreochromis niloticus". In: Marine and 
freshwater behaviour and physiology 50.3, pp. 219-232. DOI: 10 . $1080 / 10236244$. 2017.1363635.

O’Neill, Eilís (2014). Avocado farming straining Chilean water supplies. Santiago, Chile: Deutsche Welle (DW). URL: https://p.dw. com/p/1AaNB (visited on 05/2021).

Paiva, Elder Antonio S, Reginaldo Arruda Sampaio, and Herminia E Prieto Martinez (1998). "Composition and quality of tomato fruit cultivated in nutrient solutions containing different calcium concentrations". In: Journal of Plant Nutrition 21.12, pp. 2653-2661. DOI: 10.1080/01904169809365595.

Palley, Thomas I (2009). America's exhausted paradigm: macroeconomic causes of the great recession. Tech. rep. Mimeo/New America Foundation, Washington.

Penagos, D. I. et al. (2003). "Effect of weeds on insect pests of maize and their natural enemies in Southern Mexico". In: International Journal of Pest Management 49.2, pp. 155-161. DOI: 10.1080/0967087021000043111.

Poulsen, Melissa N et al. (2015). “A systematic review of urban agriculture and food security impacts in low-income countries". In: Food Policy 55, pp. 131-146. DOI: 10.1016/j.foodpol.2015.07.002.

Prenger, JJ, RP Fynn, and RC Hansen (2002). "A comparison of four evapotranspiration models in a greenhouse environment". In: Transactions of the ASAE 45.6, p. 1779. DOI: $10.13031 / 2013.11429$.

Python Software Foundation (2020). Python 3.7.9 documentation. URL: https: // docs. python.org/3.7/ (visited on 05/2021).

Rafiee, Gholamreza and Che Roos Saad (2005). "Nutrient cycle and sludge production during different stages of red tilapia (Oreochromis sp.) growth in a recirculating aquaculture system". In: Aquaculture 244.1-4, pp. 109-118. DOI: 10 . 1016/ j. aquaculture.2004.10.029.

Raissi, T., N. Ramdani, and Y. Candau (2004). "Set membership state and parameter estimation for systems described by nonlinear differential equations". In: Automatica 40.10, pp. 1771 -1777. DOI: 10.1016/j . automatica. 2004.05.006.

Raïssi, Tarek, Nacim Ramdani, and Yves Candau (2009). "Set membership parameter estimation in the frequency domain based on complex intervals". In: International Journal of Control, Automation and Systems 7.5, p. 824. DOI: 10.1007/s12555009-0515-y.

Rakocy, James E et al. (2004). "Intensive tank culture of tilapia with a suspended, bacterial-based, treatment process". In: New Dimensions on Farmed Tilapia: Proceedings of the Sixth International Symposium on Tilapia in Aquaculture, pp. 584-596.

Rakocy, James E, Michael P Masser, and Thomas M Losordo (2006). "Recirculating aquaculture tank production systems: aquaponics-integrating fish and plant culture". In: SRAC publication 454. 
Randall, DJ (2011). “The Gut, Nitrogenous Waste Balance". In: In book: Encyclopedia of Fish Physiology, by Anthony P. Farrel Vol. 2.

Raviv, Michael and J Heinrich Lieth (2007). Soilless culture: theory and practice. Elsevier.

Resh, Howard M (2016). Hydroponic food production: a definitive guidebook for the advanced home gardener and the commercial hydroponic grower. 7th ed. CRC Press.

REWA (2017). Trinkwasserqualität Wasserwerk Steinhagen. Regionale Wasser- und Abwassergesellschaft Stralsund mbh. URL: https : / / www . rewa-stralsund . de / gemeinden/steinhagen/ (visited on 05/2021).

Reyes Lastiri, D. et al. (2016). "Model of an aquaponic system for minimised water, energy and nitrogen requirements". In: Water Science and Technology 74.1, pp. 3037. ISSN: 0273-1223. DOI: $10.2166 /$ wst . 2016.127.

Reyes Lastiri, Daniel (2020). Voronoi set-membership. URL: https : / / sourceforge . net/p/vorsetmembership/code/ci/master/tree/ (visited on 05/2021).

Reyes Lastiri, Daniel et al. (2018). "Model-based management strategy for resource efficient design and operation of an aquaponic system". In: Aquacultural engineering 83, pp. 27-39. DOI: 10.1016/j . aquaeng. 2018.07.001.

Riche, Martin et al. (2004). "Effect of feeding frequency on gastric evacuation and the return of appetite in tilapia Oreochromis niloticus (L.)" In: Aquaculture 234.1-4, pp. 657-673. DOI: 10.1016/j. aquaculture.2003.12.012.

Richter, H et al. (2003). "Evacuation of pelleted feed and the suitability of titanium (IV) oxide as a feed marker for gut kinetics in Nile tilapia". In: Journal of fish Biology 63.5, pp. 1080-1099. DOI: 10.1046/j .1095-8649.2003.00225 .x.

Roosta, Hamid R and Mohsen Hamidpour (2013). “Mineral nutrient content of tomato plants in aquaponic and hydroponic systems: Effect of foliar application of some macro-and micro-nutrients". In: Journal of plant nutrition 36.13, pp. 2070-2083. DOI: $10.1080 / 01904167.2013 .821707$.

Ruijs, Marc (2011). Soilless culture in Dutch greenhouse tomato. History, economics and current issues. Szentes, Hungary: Greenhouse Horticulture, WUR and LEI, WUR.

Rumschinski, Philipp et al. (2010). "Set-base dynamical parameter estimation and model invalidation for biochemical reaction networks". In: BMC systems biology 4.1, p. 69. DOI: 10.1186/1752-0509-4-69.

Sachs, Jeffrey (2008). Common wealth: Economics for a crowded planet. Penguin.

Sagener, Nicole (2017). EU remains idle on curbing imports of 'blood farm' hormones. Euractive. URL: https : / / www . euractiv . com/ section/agriculture-food / news / eu - remains - idle - on - curbing - imports - of - blood - farm - hormones / (visited on 05/2021). 
Saltelli, Andrea (2002). "Making best use of model evaluations to compute sensitivity indices". In: Computer physics communications 145.2, pp. 280-297. DOI: 10 . 1016/S0010-4655(02)00280-1.

Saltelli, Andrea et al. (2008). Global sensitivity analysis: the primer. John Wiley \& Sons.

Saltelli, Andrea et al. (2010). "Variance based sensitivity analysis of model output. Design and estimator for the total sensitivity index". In: Computer physics communications 181.2, pp. 259-270. DOI: 10.1016/j.cpc.2009.09.018.

Santos, Vander Bruno dos et al. (2008). "Exponential growth model of Nile tilapia (Oreochromis niloticus) strains considering heteroscedastic variance". In: Aquaculture 274.1, pp. 96-100. DOI: 10.1016/j . aquaculture.2007.11.005.

Schneider, Oliver et al. (2004). “Digestibility, faeces recovery, and related carbon, nitrogen and phosphorus balances of five feed ingredients evaluated as fishmeal alternatives in Nile tilapia, Oreochromis niloticus L." In: Aquaculture Research 35.14, pp. 1370-1379. DOI: 10.1111/j.1365-2109.2004.01179.x.

Seawright, Damon E, Robert R Stickney, and Richard B Walker (1998). "Nutrient dynamics in integrated aquaculture-hydroponics systems". In: Aquaculture 160.3-4, pp. 215-237. DOI: 10.1016/S0044-8486(97)00168-3.

Serpa, Dalila et al. (2013). "Modelling the growth of white seabream (Diplodus sargus) and gilthead seabream (Sparus aurata) in semi-intensive earth production ponds using the Dynamic Energy Budget approach". In: Journal of sea research 76, pp. 135-145. DOI: 10.1016/j . seares.2012.08.003.

Shiyab, Safwan M et al. (2013). "Growth, nutrient acquisition, and physiological responses of hydroponic grown tomato to sodium chloride salt induced stress". In: Journal of plant nutrition 36.4, pp. 665-676. DOI: 10 .1080/01904167 .2012.754037.

Slinkert, T (2015). "Modelling of an integrated plant-fish system”. BSc thesis. Wageningen, the Netherlands: Wageningen UR. URL: https : / / edepot . wur.nl / 361048 (visited on 05/2021).

Smith, P. et al. (2014). "Agriculture, Forestry and Other Land Use (AFOLU)". In: IPCC. Climate Change 2014: Mitigation of Climate Change. Cambridge University Press, Cambridge, United Kingdom and New York, NY, USA.

Sobol, Ilya M (2001). "Global sensitivity indices for nonlinear mathematical models and their Monte Carlo estimates". In: Mathematics and computers in simulation 55.1-3, pp. 271-280. DOI: 10.1016/S0378-4754(00)00270-6.

Sonneveld, Cees and Wim Voogt (2009). Plant nutrition of greenhouse crops. Springer.

Stanghellini, Cecilia (1987). "Transpiration of greenhouse crops: an aid to climate management". PhD thesis. IMAG.

Su, Xiaodi, Laura Sutarlie, and Xian Jun Loh (2020). "Sensors, biosensors, and analytical technologies for aquaculture water quality". In: Research 2020. DOI: 10 . 34133/2020/8272705. 
Suhl, Johanna (2015). Personal communication. IGB.

Suhl, Johanna et al. (2016). "Advanced aquaponics: Evaluation of intensive tomato production in aquaponics vs. conventional hydroponics". In: Agricultural Water Management 178, pp. 335-344. DOI: 10.1016/j . agwat.2016.10.013.

Summerfelt, ST, J Davidson, and MB Timmons (2000). "Hydrodynamics in the 'Cornelltype' dual-drain tank". In: Proceedings of the third international conference of recirculating aquaculture. Roanoke, Virginia, pp. 20-22.

Summerfelt, Steven $\mathrm{T}$ et al. (2004). "A partial-reuse system for coldwater aquaculture". In: Aquacultural Engineering 31.3-4, pp. 157-181. DOI: 10.1016/j . aquaeng . 2004.03 .005$.

The SciPy community (2018a). Random sampling. URL: https : / docs . scipy . org / doc/numpy-1.14.0/reference/routines . random. html (visited on 05/2021).

- (2018b). SciPy cdist. URL: https : / / docs . scipy . org/doc/scipy/reference/ generated/scipy . spatial. distance. cdist.html (visited on 05/2021).

- (2018c). SciPy Voronoi. URL: https : / / docs . scipy . org/doc/scipy-0 . 18 .1/ reference/generated/scipy. spatial. Voronoi.html (visited on 05/2021).

- (2021). Random generator. URL: https : / / numpy . org/doc/stable/reference / random/generator. html\#numpy . random. Generator (visited on 05/2021).

Timmons, Michael Ben and James M Ebeling (2010). Recirculating aquaculture. 2nd ed. Ithaca, NY (USA) Cayuga Aqua Ventures.

Tuna, A. Levent et al. (2007). "The effects of calcium sulphate on growth, membrane stability and nutrient uptake of tomato plants grown under salt stress". In: Environmental and Experimental Botany 59.2, pp. 173 -178. ISSN: 0098-8472. DOI: 10.1016/j. envexpbot. 2005.12.007.

Turcios, Ariel E and Jutta Papenbrock (2014). "Sustainable treatment of aquaculture effluents-what can we learn from the past for the future?" In: Sustainability 6.2, pp. 836-856. DOI: 10.3390/su6020836.

UN (2015). "Transforming our world: The 2030 agenda for sustainable development". In: URL: https : / / sdgs . un . org / publications / transforming - our world-2030-agenda-sustainable-development-17981 (visited on 05/2021).

USDA (2018). Grain: world markets and trade. United States Department of Agriculture, Foreing Agricultural Service.

Uusitalo, Laura et al. (2015). "An overview of methods to evaluate uncertainty of deterministic models in decision support". In: Environmental Modelling $\mathcal{E}$ Software 63, pp. 24-31. DOI: 10.1016/j . envsoft.2014.09.017.

Van Leeuwen, SPJ et al. (2009). "Halogenated contaminants in farmed salmon, trout, tilapia, pangasius, and shrimp". In: Environmental science \& technology 43.11, pp. 40094015. DOI: $10.1021 /$ es803558r. 
Van Santen, Rutger, Djan Khoe, and Bram Vermeer (2010). 2030: Technology that will change the World. Oxford University Press.

Van Straten, Gerr T and Karel J Keesman (1991). “Uncertainty propagation and speculation in projective forecasts of environmental change: A lake-eutrophication example". In: Journal of Forecasting 10.1-2, pp. 163-190. DOI: 10.1002/f or . 3980100110.

Van Trung, Dinh et al. (2011). "Development of a nutritional model to define the energy and protein requirements of tilapia, Oreochromis niloticus". In: Aquaculture 320.1, pp. 69-75. DOI: 10.1016/j . aquaculture.2011.07.029.

Verdegem, M. C. J., R. H. Bosma, and J. A. J. Verreth (2006). “Reducing Water Use for Animal Production through Aquaculture". In: International Journal of Water Resources Development 22.1, pp. 101-113. DOI: $10.1080 / 07900620500405544$.

Visserijnieuws (Jan. 2014). Mogelijke terugvordering subsidiegelden tilapiaproject. organization. URL: https://www.visserijnieuws.nl/nieuws/mogelijke-terugvorderingsubsidiegelden-tilapiaproject (visited on 05/2021).

Volkmar, K. M., Y. Hu, and H. Steppuhn (1998). "Physiological responses of plants to salinity: A review". In: Canadian Journal of Plant Science 78.1, pp. 19-27. DOI: 10.4141/P97-020.

Walter, Eric and Hélène Piet-Lahanier (1990). “Estimation of parameter bounds from bounded-error data: a survey". In: Mathematics and Computers in simulation 32.5-6, pp. 449-468. DOI: 10.1016/0378-4754(90)90002-Z.

Wiegand, Mascha (2019). "Aquaponics development in the Netherlands". MSc thesis. Utrecht, the Netherlands: Utrecht University. URL: https: // dspace . library . uu.nl/handle/1874/393765 (visited on 05/2021).

Wik, Torsten EI, Björn T Lindén, and Per I Wramner (2009). “Integrated dynamic aquaculture and wastewater treatment modelling for recirculating aquaculture systems". In: Aquaculture 287.3-4, pp. 361-370. DOI: 10 . 1016/ j . aquaculture. 2008.10 .056$.

WUR (2019). Spotlight on Young Farmer Symposium. Farming Systems Ecology WUR, Global Network of Lighthouse Farms, and The Eurepean Coincil of Young Farmers.

Yamazaki, Hiromichi et al. (1999). "Effect of calcium concentration in nutrient solution before and after inoculation with Ralstonia solanacearum on resistance of tomato seedlings to bacterial wilt". In: Soil science and plant nutrition 45.4, pp. 1009-1014.

Yeats, P. A. et al. (2005). "Lithium-Normalized Zinc and Copper Concentrations in Sediments as Measuresof Trace Metal Enrichment due to Salmon Aquaculture". In: Environmental Effects of Marine Finfish Aquaculture. Ed. by Barry T. Hargrave. Berlin, Heidelberg: Springer Berlin Heidelberg, pp. 207-220. ISBN: 978-3540-31490-5. DOI: 10.1007/b136011. 
Yi, Yang (1998). "A bioenergetics growth model for Nile tilapia (Oreochromis niloticus) based on limiting nutrients and fish standing crop in fertilized ponds". In: Aquacultural Engineering 18.3, pp. 157-173. DOI: 10.1016/S0144-8609 (98)000284.

Zahedifar, M et al. (2012). "Influence of nitrogen and salinity levels on the fruit yield and chemical composition of tomato in a hydroponic culture". In: Journal of plant nutrition 35.14, pp. 2211-2221. DOI: 10.1080/01904167.2012.724497.

Zhai, $\mathrm{T}$ et al. (2004). "Parameter estimation for process-oriented crop growth models". In: Transactions of the ASAE 47.6, p. 2109. DOI: 10.13031/2013.17796. 



\section{Summary}

This thesis presents the development of mathematical models of water and nutrient balances in aquaponic systems, aimed at improving our understanding of this production method towards an efficient use of resources.

Our current food production systems have large social and environmental impacts, which are commonly externalized in the context of industrial production and global trade. Aquaponics is an option for sustainable food production that combines aquatic species (aquaculture) with soilless plants (hydroponics). The main challenge in aquaponics consists of balancing optimal conditions for multiple species: aquatic organisms, plants and beneficial micro-organisms. To facilitate achieving this balance, decoupled aquaponic systems have been proposed, consisting of two separate recirculating water loops. In this way, the production loop for fish operates independently from the production loop for plants, each with their own optimal conditions. Nutrient-rich water from the fish loop can be supplied to the plants continuously or at selected times, reducing fertilizer requirements. Similarly, water from the plants loop can be recovered and supplied to the fish, reducing fresh water demand.

Managing water and nutrient exchange in aquaponic systems requires continuous information about the concentrations of chemical elements (nutrients). Some nutrients, like nitrogen and phosphorus, are excreted in abundance by fish and can be used by plants. Other nutrients, like calcium and sodium, are also present in fish water, but they can easily reach harmful levels for plants. Sensors to continuously monitor these concentrations are costly and thus uncommon in commercial applications. Therefore, mathematical models combined with available measurements (semi-physical models), are suitable to support management and control of aquaponic systems.

Mathematical models for aquaculture and hydroponic systems are widely available in literature. However, they do not describe the dynamics of nutrients. Furthermore, measurements of nutrients in the components of aquaponic systems are not always available and typically show large uncertainties. 
In this context, further elaborated in Chapter 1 - General introduction, the objective of this thesis was to investigate the dynamics of water and nutrient balances in decoupled aquaponic systems and their uncertainties. The research questions formulated for this purpose aim at identifying the main challenges and opportunities in closing water and nutrient cycles, and in modelling nutrient balances under uncertainties. Chapters 2 and 3 present system-level studies. Chapters 4 and 5 focus on mathematical modelling under uncertainty, applied to biological production systems.

Chapter 2 presents a model-based study coupling a recirculating aquaculture system (RAS) for tilapia with a nutrient film technique (NFT) hydroponic system for tomato. The simulation results show that fish can provide $26 \%$ of the nitrogen requirements for plants. Furthermore, it is shown that variations in nitrate concentrations in the fish loop, can be decreased by 35\%, using a water management strategy that sends water from fish to plants based on amounts proportional to the fish feed.

Chapter 3 presents a model-based study supported by a demonstration aquaponic system in Abtshagen, Germany. The model helped identifying imbalances in the system design. It predicted excess concentrations of total suspended solids (TSS) for fish, and excess sodium, calcium, magnesium, and ammonium for plants. The model was used to develop an improved management strategy, preventing excess TSS, sodium, and ammonium, decreasing fertilizer requirements, and achieving water efficiencies similar to commercial aquaculture and hydroponic systems.

Chapter 4 presents an algorithm proposed for the calibration of mathematical models applied to biological production systems. The algorithm is based on the setmembership approach, which is a suitable alternative to statistical methods in the presence of limited and uncertain experimental data. The algorithm uses Voronoi tessellation, a novel method that characterises the feasible parameter space with (hyper)spheres, quantifying deviations from experimental data.

Chapter 5 studied the dynamics and uncertainties fish growth and excretion of macronutrients. A model was developed and calibrated using the Voronoi setmembership algorithm. Uncertainty propagation was assessed using Monte Carlo simulations. The model prediction explained $84 \%$ of the literature data variability for fish growth, $75 \%$ for digestive system content, and $28 \%$ for ammonia excretion rate. The uncertainty assessment helped identifying suitable experimental conditions for future research.

Chapter 6 - General discussion, reflects on the research questions, explaining the limitations identified in each research chapter, and suggestions for future research. 
From a system design perspective, closing water and nutrient cycles requires a comprehensive quantitative analysis, using indicators that reflect the trade-offs occurring when trying to balancing optimal conditions between the fish and plant loops. A list of suggested indicators for future research is provided. From a system operation perspective, several specific problems and proposed solutions were identified. Solids should be removed from fish water by combining mechanical filtration and sedimentation, to better regulate water exchange from fish to plants, and to stabilize nutrient concentrations for plants. Water storage between fish and plant loops should be aerated to prevent nitrogen loss via denitrification. Fish feed should be selected based on the mineral concentrations in local fresh water, to prevent excess of nutrients harmful for plants, which decrease the potential for nutrient reuse.

There is high multidisciplinarity involved in model development of aquaponic systems. Studies with a gradual increase in complexity are thus suggested. Future research on nutrient balances, for example, could consider the chemical form of nutrients and their bioavailability for fish and plants. Mathematical models of aquaponic systems and their components should consider simultaneously multiple variables with multiple time scales.

The discussion is extended to the more general field of modelling biological production systems, providing a framework for the development of mathematical models under uncertainty, combining set-membership calibration with uncertainty propagation assessment, to identify critical variables for future experimental measurements.

Finally, the discussion is brought back to the general socio-economic context. It is suggested to investigate whether the risks of aquaponics are most sensitive to the fish production loop. If that is the case, financial incentives for production should focus on the expansion of existing aquaculture farms to include hydroponic production. Furthermore, it is necessary to improve communication between researchers and producers. 



\section{Acknowledgements}

I wish to tank my PhD supervisors Karel, Hans and Huub, for their support, all the way to the last rush after a few years of hiatus, long after my contract. Support that was extended by my current supervisors and colleagues at Farm Technology, Peter, Simon, and Rachel, who encouraged me and covered a large chunk of my workload so that I could make the last thesis stretch. Thus, gratitude is extended to them.

Professionally, all the years of contact with colleagues from BCT, ETE, FTE, HPP, FSE, and Entomology, has been an enriching experience beyond measure.

Now, on the personal side of things, I prefer to try and lead my life letting people know what they mean to me in person. On top of that, as usual, I'm writing these lines a few hundred degrees of planetary rotation close to the deadlines (whether before or after the lines, I'm not fully sure), and my shoulder hurts from the second vaccine shot against that nasty flu everyone keeps talking about, so I really don't feel like spending any more time in a document that, well, $\mathrm{y}^{\prime}$ all know what I think.

Love to all. 



\section{SENSE}

Netherlands Research School for the

Socio-Economic and Natural Sciences of the Environment

\section{I P L O M A \\ for specialised PhD training}

The Netherlands research school for the Socio-Economic and Natural Sciences of the Environment (SENSE) declares that

\section{Daniel Reyes Lastiri}

born on $2^{\text {nd }}$ July 1985 in Queretaro, Mexico

has successfully fulfilled all requirements of the educational PhD programme of SENSE.

Wageningen, 7 September 2021
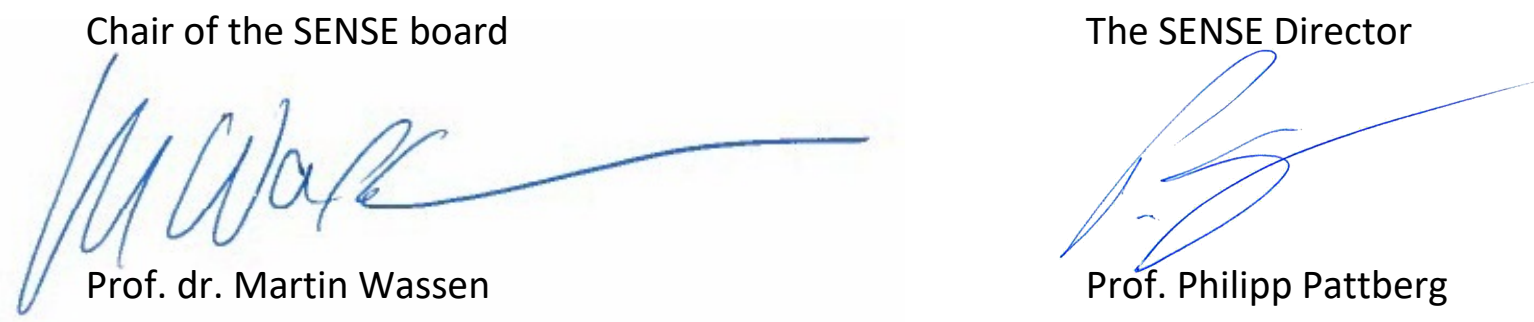

The SENSE Research School has been accredited by the Royal Netherlands Academy of Arts and Sciences (KNAW)

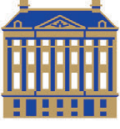

$\begin{array}{llllllllllllllllllllll}K & O & N & I & N & K & L & I & J & K & E & N & E & D & E & R & L & A & N & D & S & E\end{array}$ $\begin{array}{lllllllllllllllllllllllll}\text { A } & K & A & D & E & M & I & E & V & A & N & W & E & T & E & N & S & C & H & A & P & P & E & N\end{array}$ 


\section{SENSE}

The SENSE Research School declares that Daniel Reyes Lastiri has successfully fulfilled all requirements of the educational PhD programme of SENSE with a work load of $44.0 \mathrm{EC}$, including the following activities:

\section{SENSE PhD Courses}

- Environmental research in context (2016)

- Techniques for writing and presenting a scientific paper (2016)

- Research in context activity: 'Organising a Python course on mathematical modelling for Urban Systems Engineering' (2018)

\section{Other PhD and Advanced MSc Courses}

- Physical modelling, Wageningen University (2014)

- Modelling dynamic systems, Wageningen University (2014)

- Parameter estimation and model structure identification, Wageningen University (2015)

o System identification in the life sciences, DISC (2017)

- Exploring teaching outside academia, Wageningen Graduate Schools (2017)

\section{External training at a foreign research institute}

- Foreign stay INAPRO aquaponics project partners, China Agricultural University, China (2014)

- COST training school in aquaponics, LEGTPA Lozère, France (2017)

\section{Management and Didactic Skills Training}

- Co-organizing workshop BCT - Shouguang Vegetable Industry (2015)

- Organizing meeting with Japanese entrepreneurs at WUR (2017)

- Supervising three BSc students with theses titled 'Modelling of an integrated plant-fish system' (2015), Modelling excretion of Nile tilapia (Oreochromis niloticus) in a recirculating aquaculture system; dynamic system approach (2017) and 'Modelling the effects of excretion variations on nutrient dynamics in a recirculating aquaculture system' (2018)

- Supervising two MSc students with thesis titled 'Dynamic model of an INAPRO demonstration aquaponic system' (2016) and 'Dynamic model for nutrient uptake by tomato plant in hydroponics' (2016)

- Teaching assistant in the BSc course 'Physical transport phenomena' (2015) and MSc course 'Modelling of biobased production systems' (2015)

\section{Oral Presentations}

- Numerical optimisation of water, nutrient and energy requirements in an aquaponic system. New developments in IT and water, IWA, 09 February 2015, Rotterdam, The Netherlands

SENSE coordinator PhD education

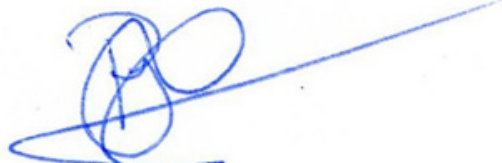

Dr. ir. Peter Vermeulen 

The research described in this thesis was financially supported by the INAPRO project, with funding from the European Union's Seventh Framework Programme for research, technological development and demonstration under grant agreement no. 619137.

Financial support from Wageningen University and the INAPRO project for printing this thesis is gratefully acknowledged.

Printed by proefschriftmaken.nl 